\title{
Nanomagnetic memory and logic: energy-delay-reliability trade-off
}

A Dissertation
Presented to
the Faculty of the School of Engineering and Applied Science
University of Virginia
In Partial Fulfillment
of the requirements for the Degree
Doctor of Philosophy (Electrical and Computer Engineering)

by

Kamaram Munira

December 2012 



\section{Approval Sheet}

This Dissertation is submitted in partial fulfillment of the requirements for the degree of Doctor of Philosophy (Electrical and Computer Engineering)

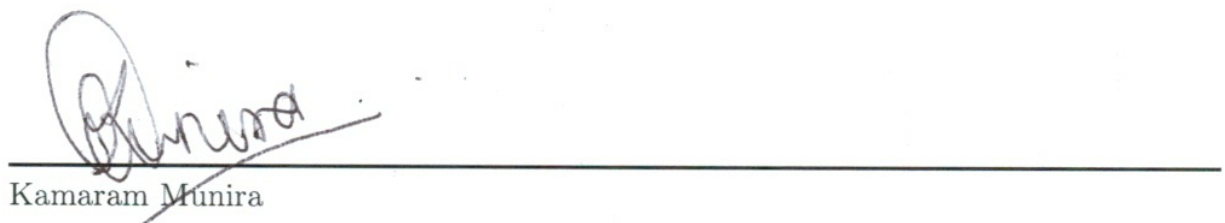

This Dissertation has been read and approved by the Examining Committee:
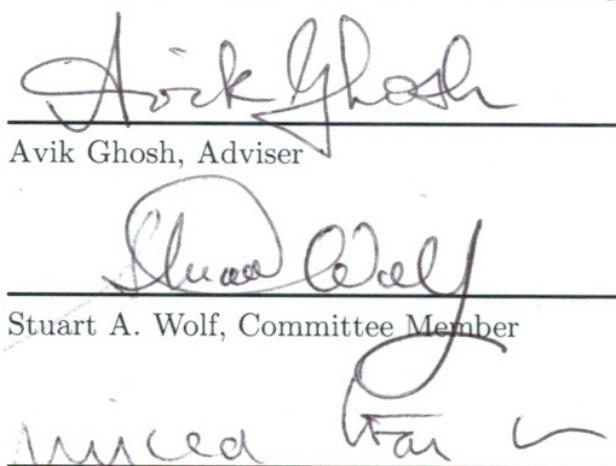

Mircea R. Stan, Committee Member
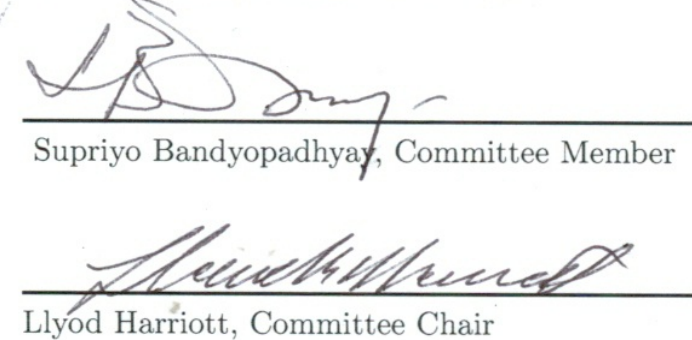

Accepted for the School of Engineering and Applied Science:

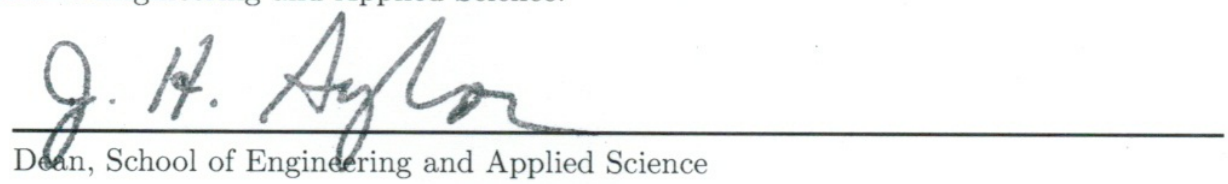

December 2012 


\section{Acknowledgement}

I owe my gratitude to a great many people who have made this dissertation possible and because of whom my graduate experience has been one that I will cherish forever.

My deepest gratitude is to my advisor, Dr. Avik Ghosh. I have been amazingly fortunate to have an advisor who gave me the freedom to explore on my own, and at the same time the guidance to recover when my steps faltered. Dr. Ghosh taught me how to question thoughts and express ideas and turned me from a silly undergrad to a researcher.

I am also indebted to the members of the Spin-Transfer Torque RAM research team with whom I have interacted during the course of my graduate studies. Particularly, I would like to acknowledge Dr. Stuart Wolf, Dr. Jiwei Lu, Dr. William Butler, Dr. Claudia Mewes, Dr. Eugene Chen, Dr. Mircea Stan, Dr. Dmytro Apalkov, Dr. Pieter Visscher and Anurag Nigam for the many valuable discussions that helped me understand my research area better. I would also like to thank the Multiferroics logic research team, Dr. Jayasimha Atulasimha, Dr. Supriyo Bandyopadhyay, Mohammad Fashemi and Kuntal Roy.

I am also grateful to all my colleagues in the VINO research group, especially Frank Tseng, Carlos Polanco and Dincer Unluer. Thank you for making me laugh every single day and being there for me.

I am also grateful to the following former or current staff at Charles L. Brown department of Electrical and Computer engineering at University of Virginia, for their various forms of support during my graduate study.

Many friends have helped me stay sane through these difficult years. Their support and care helped me overcome setbacks and stay focused on my graduate study. I greatly value their friendship and I deeply appreciate their belief in me. I want to give special thanks to my two best friends, Anwarul Mir and Tajruba Dilshad.

Most importantly, none of this would have been possible without the love and patience of my family. I dedicate this dissertation is to them: Amma, Abba, my brother Nawshad, sister Labiba and my two darling nieces, Lamisa and Aaliyah. They have been a constant source of love, concern, support and strength all these years. I would like to express my heart-felt gratitude to my family. 
Finally, I appreciate the financial support from DARPA, Samsung/Grandis, NRI, SRC, and NRI that funded parts of the research discussed in this dissertation. 


\section{Abstract}

The ability to rotate the magnetization of a single domain nanomagnet using spin polarized current or uniaxial strain leads to exciting possibilities for low-power embedded memory and logic applications. Realizing those applications for real life usage requires addressing a complex and interlinked set of problems: material properties of the ferromagnet-oxide heterostructure, spin transport, micromagnetics and thermal stochasticity of the free layer. A particular challenge the STT-RAM industry faces is maintaining a high thermal stability while trying to switch within a given voltage pulse with an acceptably low error rate and energy cost. While operating at lower barrier increases the static error in STT-RAMs, it decreases the dynamic write error rate associated with the spins freezing around stagnation points along the potential energy landscape of the nanomagnets. We introduce a comprehensive and predictive STT-RAM modeling platform that operates at different levels of complexities, ranging from a quasi-analytical model for the energy-delay-reliability trade-offs to a fully atomistic, chemistry based multi-orbital model for predictive material design and optimization. Using this platform, we identify suitable alloys for perpendicular, in-plane and partially perpendicular magnets, identify the advantages and trade-offs with double barrier junctions, and underscore the dual role of thermal fluctuations, both in hindering rotation and also in releasing spins from their stagnation points. A similar set of challenges confronts 'straintronics based multiferroic logic, where once again thermal perturbations play a decisive role on the dynamic writing error rate. In presence of stagnation points, applied stress, demagnetization field and dipole-dipole interactions, the error rate and switching delay can be controlled by material design and by engineering the stress profile on the nanomagnets. 


\section{Contents}

Acknowledgement $\quad$ ii

Abstract

Contents

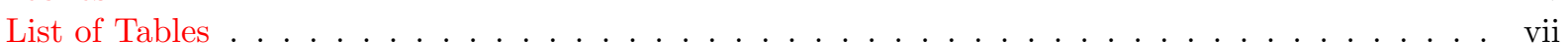

List of Figures . . . . . . . . . . . . . . . . . . . . . viii

1 Introduction 1

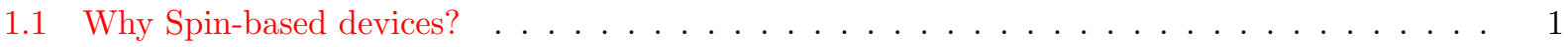

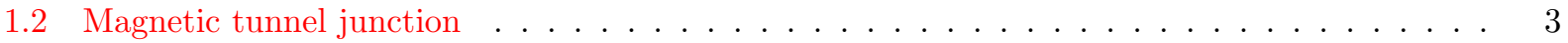

1.3 Spin-transfer torque RAM $\ldots \ldots \ldots \ldots \ldots \ldots \ldots \ldots \ldots$

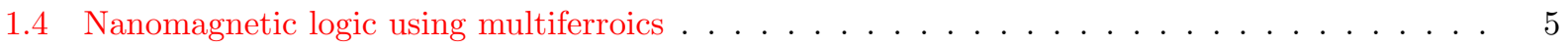

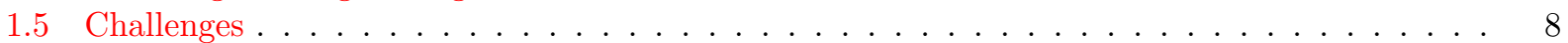

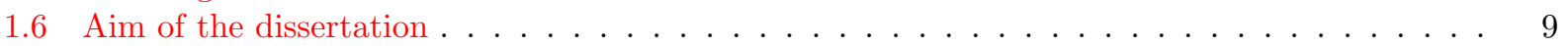

2 Unified model for STT-RAM $\quad 10$

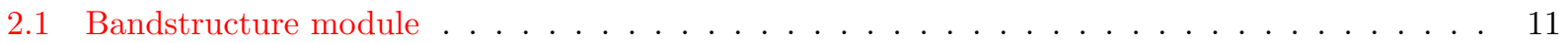

2.1 .1 Fully atomistic model . . . . . . . . . . . . . . . . . . . . . . . 11

2.1.2 Parameterized continuum grid model . . . . . . . . . . . . . . . . . . . 13

2.1.3 Quasi-analytical physics based compact model . . . . . . . . . . . . . . . . . . 14

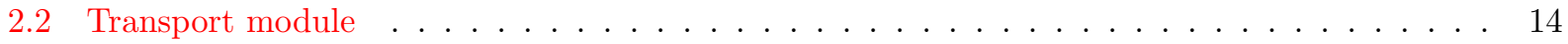

2.2.1 Non-Equilibrium Green's Function . . . . . . . . . . . . . . . . . . . . 14

2.2 .2 Modified Simmons' model . . . . . . . . . . . . . . . . . . . . . . 15

2.3 Stochastic macrospin dynamics . . . . . . . . . . . . . . . . . . 20

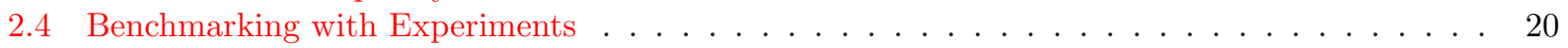

3 Investigating Spin-transfer torque switching using the unified model 24

3.1 How parallel to anti-parallel and anti-parallel to parallel

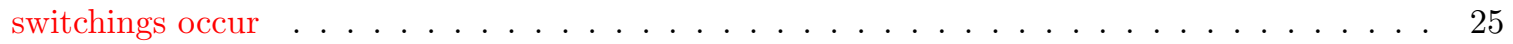

3.2 AP to $\mathrm{P}$ and $\mathrm{P}$ to $\mathrm{AP}$ switching voltages asymmetry $\ldots \ldots \ldots \ldots \ldots \ldots \ldots$

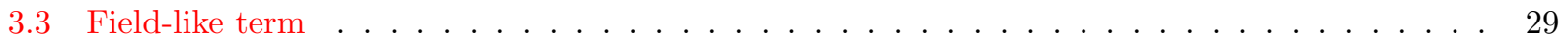

4 Comparative material issues for faster switching in STT-RAM 33

4.1 Critical switching currents and thermal stabilities . . . . . . . . . . . . . . . . 35

4.1 .1 In-plane . . . . . . . . . . . . . . . . . . . . . . . . 35

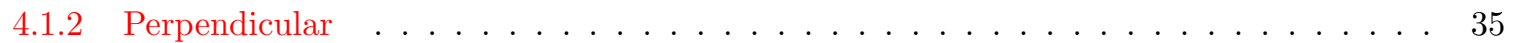

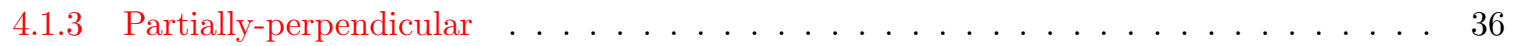

4.2 Switching speeds of different classes of free layers $\ldots \ldots \ldots \ldots \ldots$

4.2 .1 Low vs. high $H_{K}$ in-plane materials $\ldots \ldots \ldots \ldots \ldots \ldots \ldots$

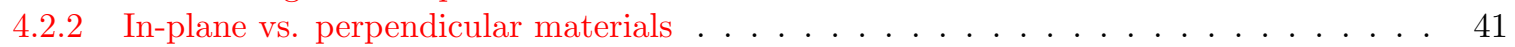

4.2 .3 In-plane vs. partially-perpendicular materials f . . . . . . . . . . . . . . 42

4.3 Material directions needed to be taken . . . . . . . . . . . . . . . . 43 
5 Energy-delay-reliability trade-off in perpendicular STT-RAM 44

5.1 Thermal fluctuation: blessing or curse ? . . . . . . . . . . . . . . . . . . . . . 46

5.2 Current needed for worst case writing . . . . . . . . . . . . . . . . . . . . . . . 47

5.3 Voltage needed for worst case writing . . . . . . . . . . . . . . . . . . . . . . 49

5.4 Worst case energy consumption and switching delay due to thermal fluctuations . . . . . . . 49

5.5 Manufacturing variations . . . . . . . . . . . . . . . . . . . . . 52

6 Energy-delay-reliability in nanomagnetic logic using multiferroics $\mathbf{5 4}$

6.1 Energy landscape of two dipole coupled nanomagnets (NM1 and NM2) . . . . . . . . . . 56

6.2 Information transmission between two NMs taking thermal perturbations into account . . . . 62

6.2.1 Switching reliability with abrupt stress removal . . . . . . . . . . . . . . 63

6.2.2 Switching reliability with tapered stress removal . . . . . . . . . . . . . . . 65

6.2.3 Switching efficiently using region II - the dipole dominated regime . . . . . . . . . . 66

6.3 Performance comparison between various stressing profiles . . . . . . . . . . . . . . 67

7 Conclusion and future work $\mathbf{6 9}$

7.1 Faster and more reliable writing using external field + spin-transfer torque . . . . . . . . . 69

7.2 Double barrier magnetic tunnel junction . . . . . . . . . . . . . . . . . . . . . 71

7.3 Alternate design FM-M-FM-I-FM . . . . . . . . . . . . . . . . . . . . 75

7.4 Combinatorial STT-RAM . . . . . . . . . . . . . . . . . . . . . . . 75

7.5 A quasi-analytical model to study energy-delay-reliability trade-off in nanomagnetic logic . . . . . . . . . . . . . . . . . . . . . 76

A Modeling magnetic tunnel junction using NEGF 77

B Atomistic study of strained $\mathrm{Fe} / \mathrm{MgO} / \mathrm{Fe} \quad \mathbf{8 4}$

B.1 Extraction of Extended Huckel theory parameters from VASP bandstructure . . . . . . . . . 84

B.2 Strained $\mathrm{Fe} / \mathrm{MgO} / \mathrm{Fe}$ tunnel junction . . . . . . . . . . . . . . . . . . . . 84

B.3 Fe $\Sigma$ calculation of $[100]$ surface . . . . . . . . . . . . . . . . . . . . . 87

B.4 Current calculation . . . . . . . . . . . . . . . . . . . . . . 92

C Publications
96

Bibliography 909 


\section{List of Tables}

4.1 A list of ferromagnetic materials that are being investigated for the free layer in STT-TAM. ॥ stands for in-plane materials while $\perp$ for perpendicular materials. . . . . . . . . . . . . .

6.1 Energy dissipation, error rate and delay comparison of various stressing profile in nanomagnetic logic using multiferroics . . . . . . . . . . . . . . . . . 68

7.1 Energy consumption during a write that ensures an error threshold of $10^{-9}$. A field is applied parallel to the spin torque current for the duration of the switching. Even though the applied external field reduces the delay, the energy consumption increases considerably . . . . . . . .

7.2 Energy consumption during a write that ensures an error threshold of $10^{-9}$. A field is applied perpendicular to the spin torque current for $0.1 \mathrm{~ns}$. The perpendicular applied external field further reduces the delay, without consuming as much energy than that used during parallel applied field. . . . . . . . . . . . . . . . . . . . 


\section{List of Figures}

1.1 Hysteresis loop of a ferromagnetic disk. The disk retains its magnetization, M, even after the removal of the applied field, H. . . . . . . . . . . . . . . . . . . . . .

1.2 (a) In CMOS technology, an energy barrier is needed to preserve a binary state. In "off" state, the barrier prevents electrons from traveling to " 0 ". When the barrier is lowered with an applied gate voltage, electrons can travel to "0". (b) Collective spin switching in a nanomagnet.

1.3 Giant magnetoresistance in a magnetic tunnel junction. When the magnetization of the free and fixed layers are in parallel configuration, the resistance across the valve is low which can be interpreted as Bit " 0 ". When the magnetization of the free and fixed layers are in anti-parallel configuration, the resistance across the valve is high which can be interpreted as Bit " 1 ".

1.4 (a) 1 Transistor - 1 MRAM cell with field induced writing, (b) 1 Transistor - STT-RAM cell , and (c) the write current as a function of cell width for field and spin-transfer torque writing

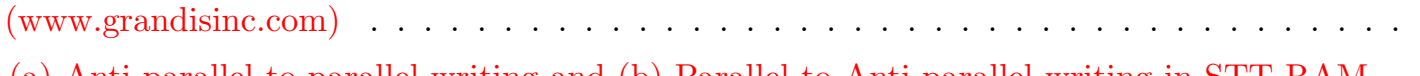

1.5 (a) Anti-parallel to parallel writing and (b) Parallel to Anti-parallel writing in STT-RAM . .

1.6 (a) In multiferroic logic, information is stored in the magnetostrictive Terfenol-D disk with an layer of piezoelectric below it. To Bennett-clock the disk, a voltage is applied to the PZT, causing is to stress. The stress is then converted to strain in the Terfenol-D, causing the magnetization to rotate. (b) Information is passed along an array of disks, which are coupled anti-ferromagnetically to each other in the ground state. (c)-(h) Information transmission from disk 1 to 3.

2.1 Fully integrated STT-RAM solver . . . . . . . . . . . . . . . . . . .

2.2 Atomistic Extended Huckel Theory + Quantum transport Strained Fe/MgO/Fe . . . . . . . 11

2.3 Mass dependent tunneling in $\mathrm{Ni} / \mathrm{MgO} / \mathrm{Ni} \mathrm{MTJ}$. . . . . . . . . . . . . . . . . . . . . . . 12

2.4 Continum grid representation of a MTJ . . . . . . . . . . . . . . . .

2.5 Band structure of a magnetic tunnel junction. The bottom of $\uparrow$ and $\downarrow$ conduction bands in ferromagnetic $(\mathrm{FM})$ contacts are separated by $\delta \mathrm{eV}$, while the insulating oxide introduces a barrier. $E_{F}$ is the Fermi level, $\mathrm{W}$ is the width of the insulating barrier and $\mathrm{U}$ is is the barrier offset between the contact and the insulator. The magnetization of the right layer is fixed, while the left layer is free to rotate. . . . . . . . . . . . . . . .

2.6 (a) Resistance vs voltage data for an in-plane $\mathrm{CoFeB} / \mathrm{MgO} / \mathrm{CoFeB}$ MTJ where the magnetization of the free layer is held constant with an external applied field. (b) The NEGF model was used to fit the experimental data and the transport parameters extracted from the fitting are $E_{F}=2.25 \mathrm{eV}, \mathrm{U}=1 \mathrm{eV}$, width $=1 \mathrm{~nm}, m_{c}=1.3, m_{\text {barr }}=0.32$ and $\delta=1.96 \mathrm{eV}$. (c) The parameters were then used to calculate torque that served as an input into the LLG solver. An $H_{K}$ of 5000e, $M_{S}$ of $1050 \mathrm{emu} / \mathrm{cc}$ and Gilbert damping of 0.02 were used. The combined model predicted the $\mathrm{P}$ to $\mathrm{AP}$ switching to take place at $-380 \mathrm{mV}$ and $\mathrm{AP}$ to $\mathrm{P}$ switching to take place at $260 \mathrm{mV}$ for a pulse width of $10 \mathrm{~ns}$ and initial angle of $0.04 \mathrm{rad}$. The switching voltage of the comprehensive model matched that of the experiment. . . . . . . . . . 
2.7 Parallel to anti-parallel switching takes place in an in-plane $\mathrm{CoFeB} / \mathrm{MgO} / \mathrm{CoFeB}$ magnetic tunnel junction at $-0.38 \mathrm{~V}$ given a $10 \mathrm{~ns}$ pulse of spin current, with a spin current density, $\eta \mathrm{J}=-28.5 \mathrm{MA} / \mathrm{cm}^{2}$. A current pulse of at least $10 \mathrm{~ns}$ is needed for switching at $-0.38 \mathrm{~V}$. Voltages greater than $-0.38 \mathrm{~V}$ will not be able to switch the free ferromagnet within $10 \mathrm{~ns}$ as shown in the figure. $H_{K}$ of $39.78 \mathrm{KA} / \mathrm{m}, M_{S}$ of $1050 \mathrm{KA} / \mathrm{m}$ and Gilbert damping of 0.02 were used. The magnetization of the free ferromagnet switches from $\theta \approx 0$ to $\theta \approx \pi . M_{Z}=\cos \theta$. The simulation results agree quantitatively with experiments. . . . . . . . . . .

2.8 Anti-parallel to parallel switching takes place in an in-plane $\mathrm{CoFeB} / \mathrm{MgO} / \mathrm{CoFeB}$ magnetic tunnel junction at $0.26 \mathrm{~V}$ given a $10 \mathrm{~ns}$ pulse of spin current, with spin current density $\eta \mathrm{J}=28.5 \mathrm{MA} / \mathrm{cm}^{2}$. A current pulse of at least $10 \mathrm{~ns}$ is needed for switching at $0.26 \mathrm{~V}$. Voltages less than $0.26 \mathrm{~V}$ will not be able to switch the free ferromagnet within $10 \mathrm{~ns}$ as shown in the figure. The magnetization of the free ferromagnet switches from $\theta \approx \pi$ to $\theta \approx 0$. The simulation results agree quantitatively with experiments. . . . . . . . . . . . .

3.1 AP to $\mathrm{P}$ switching at $0.3 \mathrm{~V}$. The free layer and insulator interface is at position $2 \mathrm{~nm}$. (a)Time $=0 \mathrm{~ns}$ : The free-layer is anti-parallel to the fixed layer, (b) Time $=2.2 \mathrm{~ns}$ : Majority electrons with respect to the fixed layer tunnel through the barrier and accumulate at the insulator-free layer interface. The accumulated majority electrons exerts a torque on the free layer which causes its magnetization to switch. (c)Time=2.6ns: The free layer switching from anti-parallel to parallel configuration. (d)Time $=5 \mathrm{~ns}$ : The final magnetization of the free layer is parallel to the fixed layer. . . . . . . . . . . . . . . . .

$3.2 \mathrm{P}$ to AP switching at $-0.4 \mathrm{~V}$. The free layer and insulator interface is at position $2 \mathrm{~nm}$. (a)Time $=0$ ns: The free-layer is parallel to the fixed layer, (b) Time $=6 n s$ : Minority electrons with respect to the free layer tunnel through the barrier, reflect back from the fixed layer and insulater interface, and accumulate at the insulator-free layer interface. The accumulated minority electrons exerts a torque on the free layer which causes its magnetization to switch. (c)Time=7.6ns: The free layer switching from parallel to anti-parallel configuration. (d)Time $=8 \mathrm{~ns}$ : The final magnetization of the free layer is anti-parallel to the fixed layer. . . .

3.3 Voltage dependent polarization. Effective polarization at the given energy, $\eta=\left(n_{\uparrow}-n_{\downarrow}\right) /\left(n_{\uparrow}+\right.$ $n_{\downarrow}$ ), where $n_{\uparrow}$ and $n_{\downarrow}$ are the number of majority and minority electrons respectively. (a) When $\Delta<E_{F}, \eta$, at $E_{F}$ is less than $100 \% . \eta(V>0)$ is greater than $\eta(V<0)$. Therefore, the effective polarization during AP to $\mathrm{P}$ switching is higher than that during $\mathrm{P}$ to AP switching. (b) When $\Delta>E_{F}, \eta$, at $E_{F}$ is $100 \% \cdot \eta(V>0)$ is equal to $\eta(V<0)$. Therefore, the effective polarization during AP to $\mathrm{P}$ switching is the same of that during $\mathrm{P}$ to AP switching. . . . .

3.4 As the fixed layer polarization $(\eta$ and $\delta$ ) increases, the spin-transfer torque, $\mathrm{A}(\mathrm{V})$ becomes more symmetric with voltage. The perpendicular torque has a quadratic dependence on the voltage, $B(V)=B_{0}+B_{1} V^{2}$. The term $B_{0}$ is the exchange coupling between the fixed and the free layers at zero voltage and intensifies as $\eta$ increases. Positive value of $\mathrm{B}(\mathrm{V})$ prefers parallel configuration in the MTJ while negative value prefers the anti-parallel configuration. When $\eta=66 \%$, B is negative near zero voltage but change to positive as voltage increases. The sign change is explained in Fig. 7. When $\eta=99 \%, \mathrm{~B}(\mathrm{~V})$ is mostly negetive. . . . . . . . .

3.5 Normalized perpendicular torque with respect to energy for (a) NEGF and (b) free electron models. According to the free electron model, the perpendicular torque, $J_{Y}$ is dependent on $\left(\kappa^{2}-k_{\uparrow} k_{\downarrow}\right) \approx-(2 m / \hbar)(U-E-(E-q V) \sqrt{1-\delta /(E-q V)}$. Torque for energy below $\delta-q V$ is negative and for energy above $\delta-q V$ is positive. For $\delta=1.96 \mathrm{eV}$ and applied voltage of zero, the torque switches sign at energy $1.96 \mathrm{eV}$. Sum of the perpendicular torque over the entire energy range is negative. For $\delta=1.96 \mathrm{eV}$ and applied voltage of $0.5 \mathrm{~V}$, the torque switches sign at energy $1.71 \mathrm{eV}$. Sum of the perpendicular torque over the entire energy range is positive. For $\delta=3 \mathrm{eV}$ and applied voltage of zero, the torque does not switch sign. Sum of the perpendicular torque over the entire energy range is negative. . . . . . . . . . . . .

3.6 AP to $\mathrm{P}$ switching in an in-plane magnetic layer. Magnetic properties of $\mathrm{CoFeB}, H_{K}$ of $500 \mathrm{Oe}$, $M_{S}$ of $1050 \mathrm{emu} / \mathrm{cc}$ and $\alpha$ of 0.02 are used. Positive field-like torque helps AP to P switching while a negative field-like torque delays the switching. $\ldots \ldots \ldots \ldots \ldots$ 
3.7 AP to $\mathrm{P}$ switching in a perpendicular magnetic layer. Magnetic properties of $\mathrm{CoPd}, H_{K}$ of $20,000 \mathrm{Oe}, M_{S}$ of $450 \mathrm{emu} / \mathrm{cc}$ and $\alpha$ of 0.1 are used. The field-like torque do not effect the switching delay. . . . . . . . . . . . . . . . . . . . . . . .

3.8 (a) AP to $\mathrm{P}$ and $\mathrm{P}$ to AP switching voltages for in-plane contacts. Magnetic properties of $\mathrm{CoFeB}, H_{K}$ of $500 \mathrm{Oe}, M_{S}$ of $1050 \mathrm{emu} / \mathrm{cc}$ and $\alpha$ of 0.02 are used. Blue line indicates switching voltages taking both spin-transfer and field-like torques into account, while the red lines account only for spin-transfer torques. For lower values of $\delta(<2.15 \mathrm{eV})$, field-like torque, being positive, hampers $\mathrm{P}$ to AP switching and helps AP to $\mathrm{P}$ switching. For higher values of $\delta(>2.15 \mathrm{eV})$, field-like torque, being negative, helps $\mathrm{P}$ to AP switching and hampers AP to $\mathrm{P}$ switching. (b) With field-like torque taken into account, voltage asymmetry, $V_{A P t o P} / V_{P t o A P}$ increases with increasing $\delta$. (c) Without the field-like torque, voltage asymmetry increases and stabilizes at 1 for higher polarization, indication symmetric spin-transfer torque switching. . . . . . . . . . .

3.9 (a) AP to $\mathrm{P}$ and $\mathrm{P}$ to AP switching voltages for perpendicular contacts. Magnetic properties of $\mathrm{CoPd}, H_{K}$ of 20,000Oe, $M_{S}$ of $450 \mathrm{emu} / \mathrm{cc}$ and $\alpha$ of 0.1 are used. The field-like torque do not effect the switching voltages and therefore, the switching voltage is dependent just on the spin-transfer torque. (b) and (c): With or without field-like torque, switching voltage becomes more symmetric with increasing $\delta \ldots \ldots \ldots \ldots \ldots \ldots$

4.1 Definition of the magnetization of the free ferromagnetic layer in a spherical coordinate system. Magnetization for (a) In-plane material: the easy axis is along the major axis, Z, of the ellipsoidal plane,. (b) Perpendicular material: the easy axis is perpendicular to the disk. (c) Partially-perpendicular: an in-plane disk is capped with a Vanadium layer that reduces the demagnetization anisotropy of the system by anti-ferromagnetic exchange coupling. In all three cases, magnetization prefers to lie near $\theta=0$ (parallel case) or $\theta=\pi$ (anti-parallel case). .

4.2 Thermal stability, $\Delta$, vs. root-mean-square, $\theta_{r m s}(\mathrm{rad})$ of the free layer nanomagnet. $\Delta$ needs to be greater than 75 for commercial applications. Root-mean-square $\theta_{r m s}(\mathrm{rad})$ identifies the initial angle that we use in our simulations. . . . . . . . . . . . .

4.3 Numerically calculated switching delay (ns) for in-plane materials with varying saturation magnetization $\left(M_{S}\right)$, anisotropy field $\left(H_{K}\right)$, and Gilbert damping (a) $\alpha=0.01$ and (b) $\alpha=0.1$. A current density of $2 \mathrm{MA} / \mathrm{cm}^{2}$ is applied. Materials that switch faster, $<10 \mathrm{~ns}$ with thermal stability $\Delta$ greater than 75 are preferred and are indicated by the area enclosed by the white boundaries. The white solid line indicates the boundary where $\Delta$ is 75 (using Eq 4.4, which defines a rectangular hyperbola) while the dashed line indicates the boundary where switching time is 10ns (using Eq 4.5, another rectangular hyperbola). Compared to materials with $\alpha=0.01$, materials with $\alpha=0.1$ rarely switch. At $\alpha=0.01$ and low magnetization, materials with low and high $H_{K}$ switch at the same speed, even though the energy barrier the spin current needs to overcome is proportional to $H_{K} M_{S}+2 \pi M_{S}^{2}$. The explanation for this is outlined in

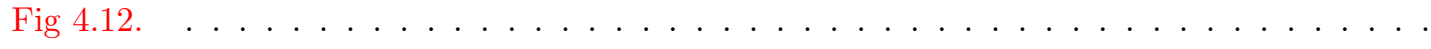

4.4 Numerically calculated switching delay (ns) for perpendicular materials with varying saturation magnetization $\left(M_{S}\right)$, anisotropy field $\left(H_{K}\right)$, and Gilbert damping : (a) $\alpha=0.01$ and (b) $\alpha=0.1$. A current density of $2 \mathrm{MA} / \mathrm{cm}^{2}$ is applied. Overall, perpendicular materials switch faster than in-plane materials, as the energy barrier the spin current needs to overcome is proportional to $H_{K} M_{S}-4 \pi M_{S}^{2}$. However, the decreased barrier has an adverse effect on thermal stability, $\Delta\left(=\left(H_{K} M_{S}-4 \pi M_{S}^{2}\right) / 2 K_{B} T\right)$. For increased $M_{S}$, thermal stability is reduced, making the range of reliable materials narrower. Most perpendicular materials have $\alpha=0.1$. Materials that switch faster $<10 \mathrm{~ns}$ and $\Delta$ greater than 75 are preferred and are indicated by the enclosed area by the white boundaries. The white solid line indicates the boundary where $\Delta$ is 75 (using Eq 4.7, which defines a rectangular hyperbola added to a linear term) while the dashed line indicates the boundary where the switching time is 10ns (using Eq 4.8). Even though higher $M_{S}$ cuts down the energy barrier, materials with high $H_{K}$ do not switch as the dominant energy barrier term is proportional to $H_{K} M_{S}$. The shape of the colored region is bounded to the left by a linear separatrix that corresponds to maintaining a perpendicular magnetization,

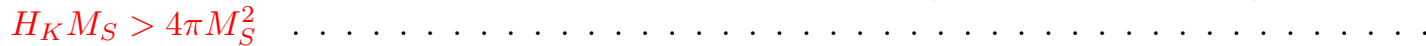


4.5 Numerically calculated switching delay (ns) for partially-perpendicular materials with varying saturation magnetization $\left(M_{S}\right)$, anisotropy field $\left(H_{K}\right)$ and $\alpha=0.01$. The demagnetization is reduced by $85 \%$ by capping. A current density of $2 \mathrm{MA} / \mathrm{cm}^{2}$ is applied. A wider range of materials (indicated by the enclosed area by the white boundaries using Eq 4.4 and 4.10) switch within $10 \mathrm{~ns}$ with thermal stability greater than 75 , as compared to in-plane materials with $\alpha=0.01$, generating thereby a more suitable class of free layer materials. . . . . . . .

4.6 Numerically calculated $H_{K}$ and $M_{S}$ combinations that switch within 10ns, overlaid with solutions of Eqs. 4.5, 4.8 and 4.10 for in-plane, perpendicular and partially-perpendicular materials. The numerical and analytical results are in excellent agreement. . . . . . . . . .

4.7 For in-plane materials with $\alpha=0.01$ and low magnetization, materials with low and high $\mathrm{H}_{K}$ switch at almost the same speed, even though the energy barrier the spin current needs to overcome is proportional to $H_{K} M_{S}+2 \pi M_{S}^{2}$. The above figure shows two cases where the saturation magnetization is fixed at (200 emu/cc), and an $\mathrm{H}_{K}$ of (a) 2000 Oe and (b) 20,000 Oe are used for the LLG simulations. At 200 Oe, the magnetization takes $0.5 \mathrm{~ns}$ to come to the equator and then another $0.4 \mathrm{~ns}$ to the south pole. At a larger $H_{K}$ of 20,000 Oe, the magnetization takes $1.03 \mathrm{~ns}$ to come to the equator but only $0.22 \mathrm{~ns}$ to come to the south pole. The asymmetry arises because the high magnetocrystalline anisotropy field hinders the magnetization while moving from the north pole to the equator, but assists it while moving from the equator to the south pole. Thus, the large time to reach the equator at higher $H_{K}$ is compensated by the faster switching from the equator to the south pole. . . . . . . . . .

4.8 Difference between (a) in-plane and (b) perpendicular magnetization switching at $2 \mathrm{MA} / \mathrm{cm}^{2}$, $H_{K}=2000 \mathrm{Oe}, M_{S}=500 \mathrm{emu} / \mathrm{cc}$ and $\alpha=0.01$. For in-plane magnetizations, both the magnetocrystalline anisotropy and demagnetization fields hinder the magnetization during the north pole to the equator transition. After passing the equator, both fields help in moving to the south pole. For perpendicular magnetizations, the anisotropy field opposes, while the demagnetization field helps the magnetization move from north pole to equator. After passing the equator, the anisotropy helps while the demagnetization field opposes the subsequent equator to south pole switching. Thus the times taken for north pole to equator and equator to south pole transitions are comparable. . . . . . . . . . . . . . .

4.9 Difference between (a) in-plane and (b) partially-perpendicular magnetization switching at $2 \mathrm{MA} / \mathrm{cm}^{2}, H_{K}=2000 \mathrm{Oe}, M_{S}=500 \mathrm{emu} / \mathrm{cc}$ and $\alpha=0.01$. The decrease in the demagnetization field causes the magnetization to switch 3 times faster for the latter case. . . . . . . . . . .

5.1 Three coupled equation blocks allow us to compute the energy consumption for a write operation in a perpendicular STT-RAM cell within a given switching time and error threshold. Equation block 1: Given a write-error-rate (WER) and delay time $\tau$, we use the non-switching expression, Eq.5.1, to extract the overdrive $I / I_{C}$ ratio, where $I$ is the current applied and $I_{C}$ is the critical switching current. Equation block 2: gives us the critical switching current $I_{C}$ using Eq. 5.2. From these two blocks, we get $I$. Equation block 3: Using a modified version of Simmons equation, Eq. 5.3, we can then estimate the switching voltage $V$ and thence the switching energy $I V \tau$. The last two steps have to be solved self-consistently, as the switching voltage for a given WER depends on current $I_{C}$, which in turn depends on the voltage-dependent polarization $\eta(V)$, which further depends on the transport parameters $E_{F}$,

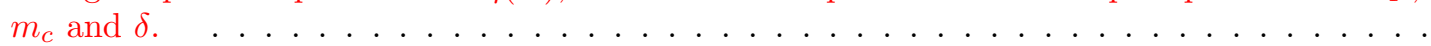

5.2 Magnetization distribution at equillibrium $\ldots \ldots \ldots \ldots \ldots \ldots$

5.3 With out thermal fluctuation, the magnetization cannnot dislogde from the stagnation point and switch. . . . . . . . . . . . . . . . . 46

5.4 Thermal fluctuations dislodge the magnetization from the stagnation point, enabling it to switch. 47

5.5 Write error rate vs. delay time at room temperature for $\Delta=60$. To attain an error rate of $10^{-9}$ within given delay times $5 \mathrm{~ns}, 10 \mathrm{~ns}$ and $20 \mathrm{~ns}$, current pulses of $3.232 I_{C}, 2.104 I_{C}$ and $1.543 I_{C}$ are needed. We use $H_{K}$ of $3.34 \mathrm{kOe}$ and $M_{S}$ of $1257.3 \mathrm{emu} / \mathrm{cc}$ for this estimate . . . . . . 
5.6 Parameter extraction from published $\mathrm{CoFeB} / \mathrm{MgO} / \mathrm{CoFeB}$ MTJ resistance-voltage curve (H. Kuboto et al, "Quantitative measurement of voltage dependence of spin-transfer torque in Mg0-based magnetic tunnel junctions with modified Simmons' mode",2001)l. The parameters extracted from the model are $E_{F}=2.2 \mathrm{eV}, U=1 \mathrm{eV}, \mathrm{W}=1 \mathrm{~nm}, m_{c}=0.3 m_{0}, m_{\text {barr }}=0.18$ $m_{0}$ and $\delta=1.98 \mathrm{eV}$. Curve $\mathrm{A}$ is for anti-parallel mode while $\mathrm{D}$ for parallel mode. $m_{0}$ is the mass of an electron. . . . . . . . . . . . . . . . . . . . .

5.7 Worst case energy consumption during a write operation at room temperature for $\Delta=60$ and error rate of $10^{-9}$. Energy consumption during (a) AP to P switching is less than that consumed during (b) P to AP switching. This is because the effective polarization of the torque at negative voltage is less than that at positive voltage. Energy consumed is the product of current, voltage applied and delay $(I V \tau)$. At lower delay, the current, and hence energy, decreases logarithmically with delay. At higher delay, the energy consumption increases linearly with delay. . . . . . . . . . . . . . . . . . . . . . .

5.8 Worst case energy consumption during a write operation at room temperature for $\Delta=60$ and error rate of $10^{-9}$ for a magnetic tunnel junction with half-metallic contacts $(\delta=3 \mathrm{eV})$. Energy consumption during AP to $\mathrm{P}$ switching is equal to that during $\mathrm{P}$ to AP switching because the effective polarization of the torque at negative voltage is same as that at positive voltage. $H_{K}=5.34 k O e$ and $M_{S}=786.5 e m u / c c \ldots \ldots \ldots \ldots \ldots$

5.9 Keeping volume and thermal stability of the free layer the same $(\Delta=60)$, the minimum energy consumption can be reduced by increased the anisotropy field, $H_{K}$ and decreasing the saturation magnetization, $M_{S}$ accordingly. The duration of the voltage pulse needed to achieve the switching with $10^{-9}$ error rate also decreases with higher anisotropy field $\alpha=0.02$. . . .

5.10 Effect of manufacturing variations on minimum energy consumption, $E_{\text {min }}$, during a $\mathrm{P}$ to AP write operation with an error threshold of $10^{-9}$. The varying parameters are effective anisotropy field $\left(H_{K}\right)$, saturation magnetization $\left(M_{S}\right)$, Gilbert damping parameter $(\alpha)$, crosssectional area of the MTJ, thickness of the free layer $(\mathrm{t})$, width of the tunneling barrier $(\mathrm{W})$, and ferromagnet-oxide interface (U). While variation in the thickness of the tunneling barrier has the most effect on the delay, cross-sectional area has the least. Mean value of the MTJ parameters used are $H_{K}=5.340 e, M_{S}=785.6 \mathrm{emu} / \mathrm{cc}, \alpha=0.02$, radius of the free layer $=20 \mathrm{~nm}, \mathrm{t}=2 \mathrm{~nm}, \mathrm{~W}=1 \mathrm{~nm}$ and $\mathrm{U}=1 \mathrm{eV} \ldots \ldots \ldots \ldots \ldots$

5.11 Corresponding minimum switching delay during a $\mathrm{P}$ to AP write operation with an error threshold of $10^{-9}$. The varying parameters are effective anisotropy field $\left(H_{K}\right)$, saturation magnetization $\left(M_{S}\right)$, Gilbert damping parameter $(\alpha)$, cross-sectional area of the MTJ, thickness of the free layer $(\mathrm{t})$, width of the tunneling barrier $(\mathrm{W})$, and ferromagnet-oxide interface $(\mathrm{U})$. .

6.1 A binary wire, spaced d nm apart. A longer wire is simplified to a 2 NMs system (NM1 and NM2). The major axis of NM1 is increased to account for the dipolar coupling to its left neighbors. . . . . . . . . . . . . . . . . .

6.2 (a) 3-step information transmission process in 2 NMs. (b) Stress profiles with abrupt removal: Cases 1 and 2, tapered: Cases 3 and 4 and reduced to Region II (to operate in an energy landscape where stress cancels out the demagnetization field and only dipolar interaction with NM1 exists): Cases 5 and 6 . The negative stress in 2, 3 and 6 allow faster switching. . . . .

6.3 (a) Below a critical NM spacing, $d=172 \mathrm{~nm}$, the magnetizations of the NMs in a semi-finite array are energetically favorable along the minor axes, losing their alternating bits ' 0 ' $(\phi=$ $\pi / 2)$ and ' 1 ' $(\phi=3 \pi / 2)$ informations. (b) The spacing between the NMs need to be more than $172 \mathrm{~nm}$ for the information stored in the Terfenol-D layer to be intact.The results in this figure is for $0 \mathrm{~K}$ temperature. . . . . . . . . . . . . . . . . . .

6.4 (a) Energy profile of NM1 with the dipolar coupling with its neighbors to the left taken into account. As the spacing between the NMs, d, is increased from $180 \mathrm{~nm}$ to $300 \mathrm{~nm}$, the energy barrier between the two stable configuration of NM1 decreases from $49.85 K_{B} T$ to $35.02 K_{B} T$. This is because the strength of the dipole coupling is proportional to $1 / d^{3}$. (b) The energy barrier of NM1 decreases with increasing spacing with its neighbors. The major axis of NM1 is adjusted accordingly in our simplified $2 \mathrm{NMs}$ system is increased to account for the dipolar coupling to its left neighbors. . . . . . . . . . . . . . . . 
6.5 The critical stresses required to Bennett-clock NM for a single NM, in $\uparrow \uparrow$ configuration with its left neighbor and in in $\uparrow \downarrow$ configuration with its left neighbor are $1.84 \mathrm{MPa}, 1.14 \mathrm{MPa}$ and $2.53 \mathrm{MPa}$ respectively at $200 \mathrm{~nm}$ separation. If stress applied is less than that of the critical stress (1.8MPa for single NM, 1MPa for $\uparrow \uparrow$ configuration with its left neighbor, $2.3 \mathrm{MPa}$ for $\uparrow \downarrow$ configuration with its left neighbor), the magnetization of the stressed NM do not switch. If stress applied is greater than that of the critical stress $(2.5 \mathrm{MPa}$ for single NM, $2 \mathrm{MPa}$ for $\uparrow \uparrow$ configuration with its left neighbor, $2.8 \mathrm{MPa}$ for $\uparrow \downarrow$ configuration with its left neighbor), the magnetization of the stressed NM switch close to its hard axis. The results in this figure is for OK temperature. . . . . . . . . . . . . . . . . . . . .

6.6 (a) Energy landscape and and changing position of the magnetization of NM2 with varied stress. At stress level below that of critical, the magnetization of NM2 is at the local minimum and initial position, $\phi=\pi / 2$. At $2 \mathrm{MPa}$, the stress applied cancels out the demagnetization field, and the dipole interaction with NM1 dominates the energy landscape. The energy minimum for $2 \mathrm{MPa}$ is near the intended destination, $\pi=\pi / 2$. However, $2 \mathrm{MPa}$ applied stress takes a long time to switch (60ns) as shown in (b) At higher stress, energy minimum shifts to the hard axis, $\phi=\pi$ and its takes a shorter time to switch. (c) $\phi$ at energy minimum for varying stresses. At Region I, for stresses below that of critical, energy minimum is at $\pi / 2$ and the magnetization doesn't switch at all. From 1.85 to 2.5MPa, region II or dipole dominated region, stress cancels out the demagnetization field and only the dipolar effect dominates the energy landscape. The minimum energy $\phi$ is close to $3 \pi / 2$ very close to the intended destination. However it takes a long time for the magnetization to switch. The high speed regions III and IV, stress starts to dominate the energy landscape and there is low and little to no dipole effect respectively. The magnetization switches pretty fast in Region III and IV . . . . . . . . . . . . .

6.7 (a) Distributions of the polar azimuthal angle $\phi$ of NM2 due to thermal fluctuations during Period A at room temperature. The mean of the distribution is at stagnation point, $\phi=\pi / 2$. (b) An applied stress of $3 \mathrm{MPa}$ for $1 \mathrm{~ns}$ is not enough to kick all the magnetization out of the stagnation point and toward the hard axis. (c) An applied stress of $4 \mathrm{MPa}$ for $1 \mathrm{~ns}$ is strong enough to kick most of the magnetization out of the stagnation point. Around the hard axes, the effect of dipole coupling with NM1 is stronger than the stress, the system therefore operating in Region III dipole effecr(Fig. 6.6c). The peak of the switched distribution is away from the hard axes, $\phi=0$ or $\pi$. (d) The peak of the distribution is along the hard axes, the system operating in Region IV dipole effect after 1 ns applied stress of $10 \mathrm{MPa}$. . . . . . . . .

6.8 Success rate of Cases 1 and 2 stressing profile. For both the profiles, success rate is lower at small stress levels, peaks around $4 \mathrm{MPa}$ and then slowly deteriorates as stress is increased. Case 2 deteriorates mush faster than 1 . The average delay of case 1 is around $1.45 \mathrm{~ns}$, while the negative stressing profile of case 2 speeds up the switching to $1.15 \mathrm{~ns} \ldots \ldots \ldots \ldots$

6.9 Comparison between cases 1 and 2 switching profiles at 10MPa. While the negative stress in case 2 speeds up the switching process, it erases some of the effect of dipolar coupling with NM1, results in a higher error rate. . . . . . . . . . . . . . . .

6.10 Success rate of Cases 3 and 4 stressing profile. While case 3 dissipates very little energy, the overall success rate is lower as the sinusoidal stress during Period B is not enough to kick all the magnetization out of the stagnation point and the negative stress during Period $\mathrm{C}$ overpowers the dipolar effect. Average switching delay is $1.2 \mathrm{~ns}$. Case 4 has better success rate with an average delay of $2.1 n s . \ldots \ldots \ldots \ldots \ldots \ldots$

6.11 Comparison between cases 3 and 4 switching profiles at 10MPa. The overall error rate is higher in case 3 as the sinusoidal stress during Period B is not enough to kick all the magnetization out of the stagnation point and the negative stress during Period $\mathrm{C}$ overpowers the dipolar effect. Case 4 has lower error rate as the gradual decrease of stress allows the magnetization switching to operate in Region II for some time. . . . . . . . . . . . . . . .

6.12 Comparison between cases 3 and 4 switching profiles at 10MPa. Operating at $1 \mathrm{~ns}$ in dipole dominated region II dipole effect results in very low error rate. Average delay for case 5 is $2.1 \mathrm{~ns}$ while the negative stress is case 6 decreases the delay to $1.55 \mathrm{~ns} . \ldots \ldots \ldots \ldots$

6.13 Operating at 1 ns in dipole dominated region II dipole effect results in very low error rate. Switching speed is increased by a negative stress in case $6 \ldots \ldots \ldots \ldots \ldots$ 
6.14 Very low error rate can be achieved by continuing to stress NM2 at 2MPa after 1ns stress of $1 \mathrm{MPa}$. Error rate of $10^{-3}$ is achieved at $10 \mathrm{~ns}$.

7.1 (a) Elliptical free layer where the magnetization prefers to lie along the major (Z) axes. The external field is applied parallel along the direction of the spin torque during writing. (b) For a given error rate, an applied field in the direction of the spin torque considerably cuts down the delay. For a field greater than 3000e, switching delay of less than 5ns is needed for an error threshold of $10^{-9} \ldots \ldots \ldots \ldots \ldots \ldots \ldots$

7.2 (a) Elliptical free layer where the magnetization prefers to lie along the major (Z) axes. The external field is applied perpendicular to the magnetization for $0.1 \mathrm{~ns}$ at the beginning of the writing. (b) For a given error rate, an applied field in the direction perpendicular to the spin torque further cuts down the switching delay compared to the case where the field was applied parallel to the spin torque. For a field greater than 5000 e, switching delay of less than $0.5 \mathrm{~ns}$ is needed for an error threshold of $10^{-9}$. . . . . . . . . . . . . . . . . . . . . 70

7.3 Fixed FM-Insulator-Free FM-Insulator-Fixed FM DMTJ system . . . . . . . . . . . . . . . . 71

7.4 (a) Van-Hove singularities in the majority band of the free layer in a DMTJ. (b) TMR ratio as a function of annealing temperature for a $\mathrm{CoFeB} / \mathrm{MgO} / \mathrm{CoFeB}(\mathrm{t} \mathrm{nm}) / \mathrm{MgO} / \mathrm{CoFeB}$ DBMTJs with various middle layer thicknesses. A TMR of $1048 \%$ is reached when the free layer is $1.2 \mathrm{~nm} 72$

7.5 (a) Large Tunnel Magnetoresistance of $1048 \%$ at Room Temperature in MgO Based Double Barrier Magnetic Tunnel Junction, l Jiang et al, Appl. Phys. Express 2 (2009) 083002. (b) Effect on TMR by free layer width modulation around $1 \mathrm{~nm}$ by numerical calculation (Non-Equilibrium Green's Function). . . . . . . . . . . . . . . . . . . . . . . . . . 73

7.6 Effect on resonance state by free layer width modulation. . . . . . . . . . . . . . . . . . . 74

7.7 Fixed FM-Metal-Free FM-Insulator-Fixed FM system . . . . . . . . . . . . . . . . . . 75

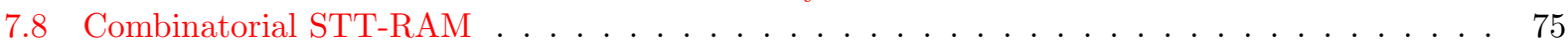

A.1 Schematic illustration of the device partitioning with matrices needed for NEGF quantum transport calculations. Magnetization direction of the drain is defined relative to the $\operatorname{source}\left(\Delta \theta=\theta_{R}-\theta_{L}\right) \quad \ldots \ldots \ldots \ldots \ldots \ldots$

A.2 Effect of inelastic scattering on tunneling magnetoresistance. . . . . . . . . . . . . . 83

B.1 Parameters for contact spin up Fe. . . . . . . . . . . . . . . . . . . . . . 85

B.2 Parameters for contact spin down Fe. . . . . . . . . . . . . . . . . . . . . . . . 85

B.3 Parameters for spin up strained $\mathrm{Fe} / 5$ [100] layers of $\mathrm{MgO} / \mathrm{Fe}$ magnetic tunneling junction. $\quad 86$

B.4 Parameters for spin down strained Fe/5 [100] layers of $\mathrm{MgO} / \mathrm{Fe}$ magnetic tunneling junction. 87

B.5 Atomistic view of a strained Fe/5 [100] layers of $\mathrm{MgO} / \mathrm{Fe}$ magnetic tunneling junction. The system is periodic in the $\mathrm{x}-\mathrm{y}$ plane. Current flows in the Z direction. . . . . . . . . . . . 88

B.6 [100] plane of Fe. Green box indicate the nearest neighbors. The red-red block represent the unit cell. . . . . . . . . . . . . . . . . . . . . . . 89

B.7 On-site and neighboring unit cells during contact Fe $\Sigma$ calculation. . . . . . . . . . . . . . . . 89

B.8 On-site atomic coordinates for contact Fe $\Sigma$ calculation in Fig. B.7. . . . . . . . . . . . . . . 90

B.9 Density of states for contact spin up Fe. . . . . . . . . . . . . . . . . . . . . . 91

B.10 Density of states for contact spin down Fe. . . . . . . . . . . . . . . . . . . . . 92

B.11 On-site atomic coordinates for strained Fe/5 [100] layers of $\mathrm{MgO} / \mathrm{Fe}$ magnetic tunneling junction. 95 


\section{Chapter 1}

\section{Introduction}

\subsection{Why Spin-based devices?}

Due to physical and electrical scaling challenges, the MOSFET-based industry (CMOS logic gates, Flash, DRAM, SRAM, etc) will reach the end of its road in the near future. Not only do the MOSFET-based devices have scalability issues, they are also facing increased power leakage and endurance problems [1, 2, 3]. Therefore, a lot of incentive is being taken today to look into alternate forms of information computation to sustain Moore's law for the next few decades. One possible solution is to store information and do logic computation by using the spin degree of freedom of electrons in ferromagnetic materials, instead of charge based operation that most MOSFET-based technologies follow [4, 5]. This dissertation will focus on two devices that utilizes the spin degree of freedom: (a) spin-transfer torque RAM (STT-RAM) and (b) nanomagnetic logic using multiferroics.

Using the spin degree of freedom over charge has two advantages: (i) non-volatility and (ii) energy-efficient collective switching of spins. Ferromagnetic materials have unpaired d orbital electrons, resulting in atoms with a net magnetic moment. Therefore, they exhibit a strong attraction to magnetic fields and are able to retain their magnetic properties after the external field has been removed. As shown in Fig. 1.1, under an applied magnetic field, the material follows a non-linear magnetization until it reaches saturation point. When the driving magnetic field drops to zero, the ferromagnetic material retains a considerable degree of magnetization. The driving magnetization field must be reversed and increased by a large value to reach saturation in the opposite direction. The ability to retain magnetization even when the magnetization field is dropped to zero or the non-volatility of the ferromagnetic materials, is useful for memory devices.

Traditional CMOS technology use electrical charge into or out of their active regions to do logic computation 


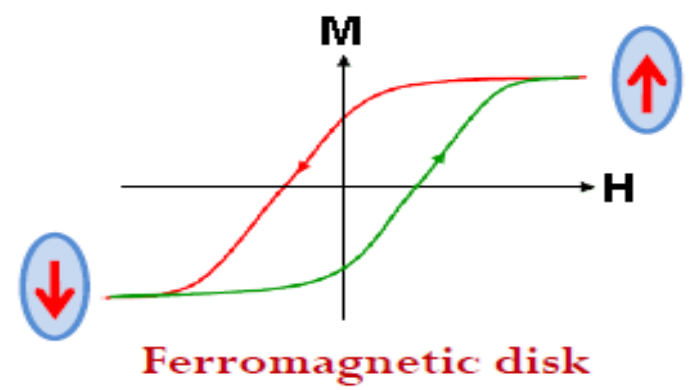

Figure 1.1: Hysteresis loop of a ferromagnetic disk. The disk retains its magnetization, M, even after the removal of the applied field, $\mathrm{H}$.

(a)
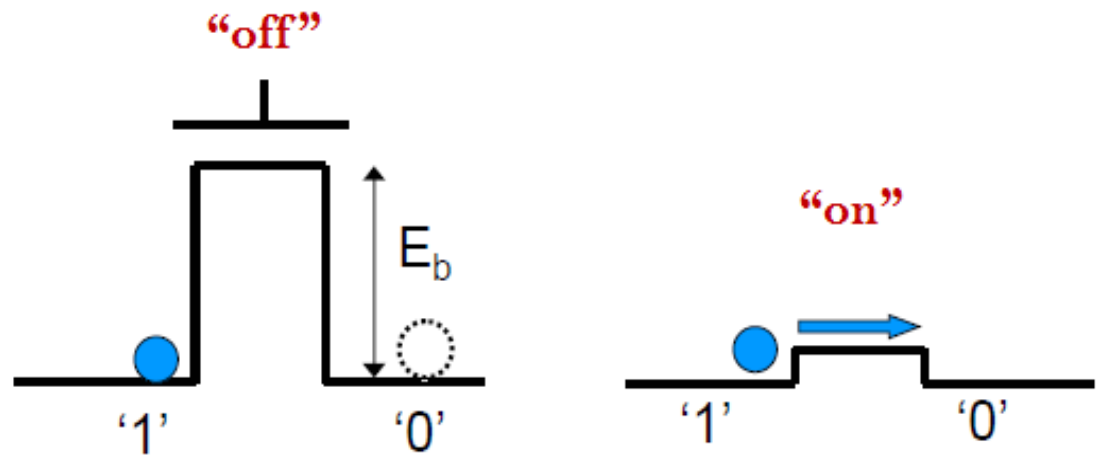

(b)

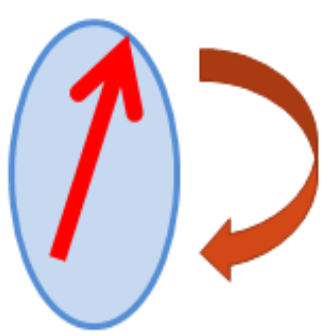

Figure 1.2: (a) In CMOS technology, an energy barrier is needed to preserve a binary state. In "off" state, the barrier prevents electrons from traveling to " 0 ". When the barrier is lowered with an applied gate voltage, electrons can travel to " 0 ". (b) Collective spin switching in a nanomagnet.

or to store information (Fig. 1.2a). An energy barrier (of height $E_{b}$ ) is need to preserve a binary state in a transistor. The barrier prevents electrons from traveling from " 1 " to state " 0 ". When the barrier is lowered with an applied gate voltage, electrons can travel to "0". A write error happens when thermal energy causes an electron to spontaneously jump over the barrier even before it is depressed. The device error probability is given by the Boltzmann factor, $\Pi_{d e v}=e^{-E_{b} / K_{B} T}$, where $\mathrm{T}$ is the temperature. The amount of energy that needs to be to depress the barrier and turn on the transistor process equal to at least $K_{B} T \ln \left(1 / \Pi_{d e v}\right)$. In 
order to support logic computation, fan in and out and interconnects, a large number of electrons $\left(\sim 10^{4}\right)$ is needed to guarantee successful communication between the binary switches, with total dissipation ending up to be $N K_{B} T \ln \left(1 / \Pi_{d e v}\right)[6]$, where $\mathrm{N}$ is the number of electrons.

On the other hand, if logic bits are encoded in two stable magnetization of the spin degree of freedom, then switching between these orientations can take place by dissipating only $\sim k T \ln \left(1 / \Pi_{d e v}\right)[7]$ of energy. Since 10,000 spins rotate for the cost of 10 due to the strong exchange coupling between the spins, the computation is intrinsically energy-efficient (Fig. $1.2 \mathrm{~b}$ ).

\subsection{Magnetic tunnel junction}

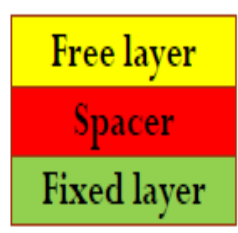

\section{Low resistance High resistance Bit "0" Bit "1"}

Figure 1.3: Giant magnetoresistance in a magnetic tunnel junction. When the magnetization of the free and fixed layers are in parallel configuration, the resistance across the valve is low which can be interpreted as Bit "0". When the magnetization of the free and fixed layers are in anti-parallel configuration, the resistance across the valve is high which can be interpreted as Bit " 1 ".

Magnetic tunnel junction (MTJ) is the building block of most spintronic devices. A MTJ [8] is basically two ferromagnetic films separated by an insulating spacer film $\left(\mathrm{MgO}, \mathrm{Al}_{2} \mathrm{O}_{3}\right)$ (Fig. 1.3). The magnetization of one ferromagnetic (FM) layer (the fixed layer) in the MTJ is pinned by exchange coupling with an antiferromagnetic layer, while the magnetization of the second (free) layer can change freely. Data in a MTJ cell can be retained in the relative spin orientations of the fixed and free FM layers (Bit " 0 " or low resistance (RP) for parallel (P) and bit "1" or high resistance (RAP) for anti-parallel (AP)). The stored data can thereafter be sensed by measuring the tunneling resistance. The data retention is non-volatile as the magnetization in the FM layers is conserved in the absence of current or applied field. Tunneling magnetoresistance $(\mathrm{TMR})$ is calculated by: $\mathrm{TMR}=(\mathrm{RAP}-\mathrm{RP}) / \mathrm{RP}[9]$. 


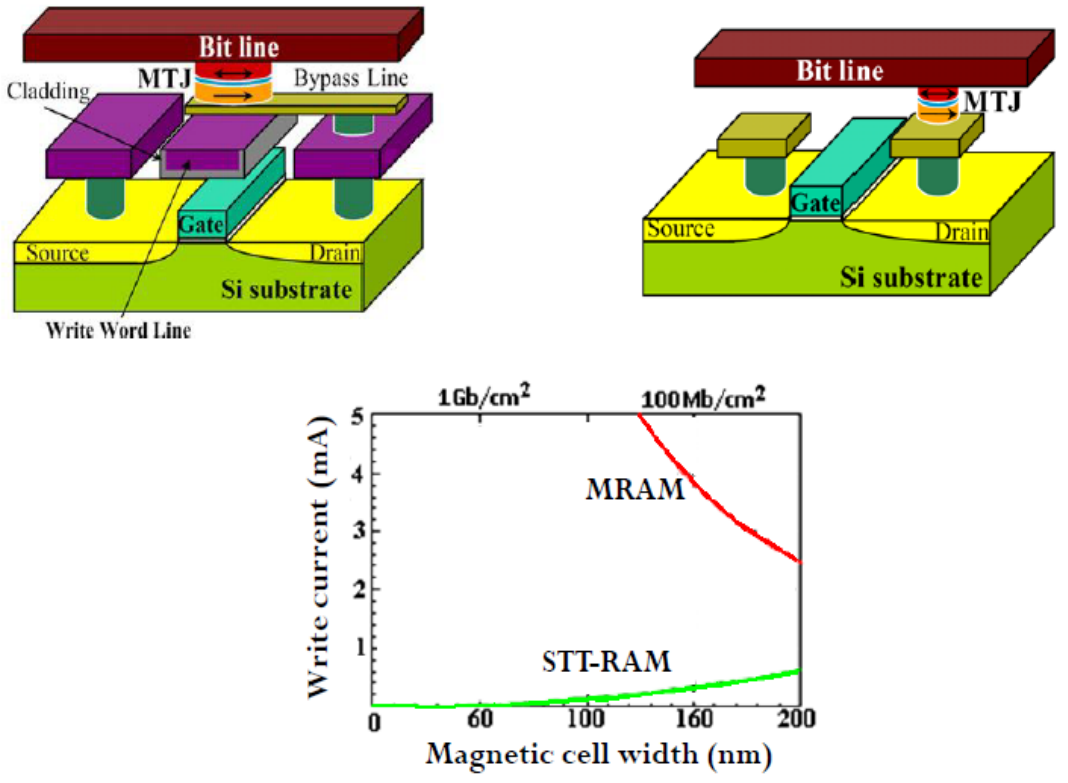

Figure 1.4: (a) 1 Transistor - 1 MRAM cell with field induced writing, (b) 1 Transistor - STT-RAM cell , and (c) the write current as a function of cell width for field and spin-transfer torque writing (www.grandisinc.com)

\section{$1.3 \quad$ Spin-transfer torque RAM}

Magnetic RAM (MRAM) (Fig. 1.4a) is the first generation random access memory that used magnetic hysteresis of the free FM layer of the MTJ to store information. A write is performed by changing the magnetic orientation of the free layer in the MTJ by an external magnetic field induced concurrently by the word and bit lines [10]. The inductive writing has high power consumption and high possibility for cross-interference as devices are scaled down to achieve higher memory density per chip (Fig. 1.4c). Fabrication is complicated as the device requires extra word lines. Therefore, it is highly unlikely that MRAM will dominate the memory industry anytime in the future.

STT-RAM (Fig. 1.4b) is one of the front-runners in spin-based memory technology today. The concept of STT-RAM was born in 1996; after theories developed by J.C. Slonczewski[11] and L. Berger[12] independently suggested that the magnetization of a thin FM film can be switched by direct transfer of spin angular momentum from a spin polarized current (via exerting torque). The current required to switch scales down with size (Fig 1.3c). When the FM layers are in AP configuration, an applied positive voltage would pass electrons through the fixed FM layer, making them fully spin-polarized in the same direction as the layer's majority electrons magnetization. After a critical current, $J_{C}$, is reached the spin polarized electrons will induce a torque on the anti-parallel electrons in the free FM layer and align the magnetization direction of the free FM layer to match its own magnetization, resulting in a MTJ in parallel configuration (Fig. 1.4a). If 


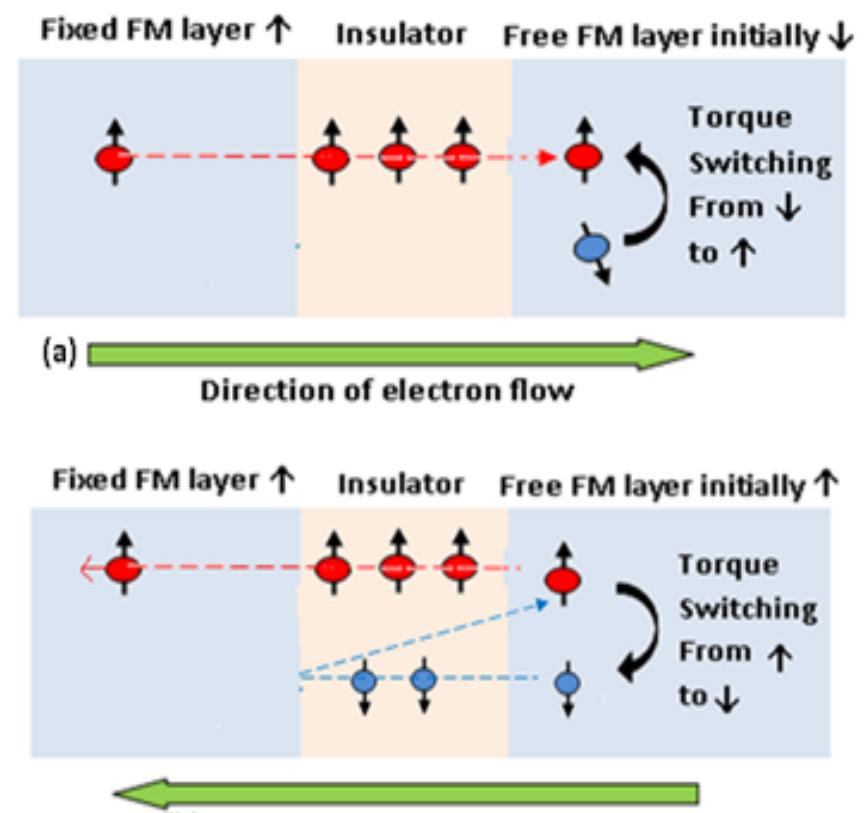

(b) Direction of electron flow

Figure 1.5: (a) Anti-parallel to parallel writing and (b) Parallel to Anti-parallel writing in STT-RAM

the current is reversed at negative voltage (electrons first passing through the free FM layer), minority down electrons with respect to fixed FM layer's magnetization, will be reflected back into the free layer, thereby aligning the soft magnetization direction from up to down, making the device anti-parallel (Fig. 1.5b).

\subsection{Nanomagnetic logic using multiferroics}

Nanomagnetic logic, where shape-anisotropic nanomagnets with two stable magnetization orientations act as binary switches, has emerged as a potential replacement for traditional transistor-based logic because magnets can be far more energy-efficient than transistors. A recent version of nanomagnetic logic - termed straintronic multiferroic logic (SML) - visualizes using voltage generated strain to rotate or switch the magnetization of multiferroic nanomagnets. This action dissipates very little energy $(<1 \mathrm{aJ})$ and is therefore particularly attractive for both logic and memory [13]. Logic architectures are configured in the same way as magnetic quantum cellular automata (MQCA) [14], where dipole interaction between nearest neighbor magnets elicits logic functionality $[15,16]$ and information is transmitted unidirectionally from one stage to the next (Fig. 1a) through inter-magnet dipole coupling and the action of a multi-phase clock. The clock acts as a Bennett clock [17] that rotates a nanomagnet's magnetization through $\sim 90^{\circ}$ prior to a bit propagating through it. Until recently, the popular means for rotating the magnetization employed either a local magnetic field or spin-transfer torque, with associated energy dissipations of $\sim 10^{9} K_{B} T[18]$ and $\sim 10^{8} K_{B} T[19,20]$, 
(a)

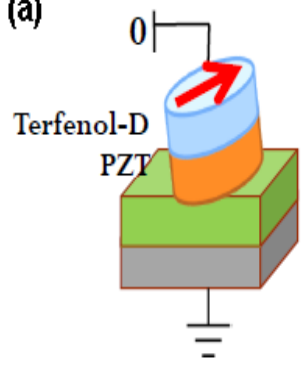

(b)

(c)

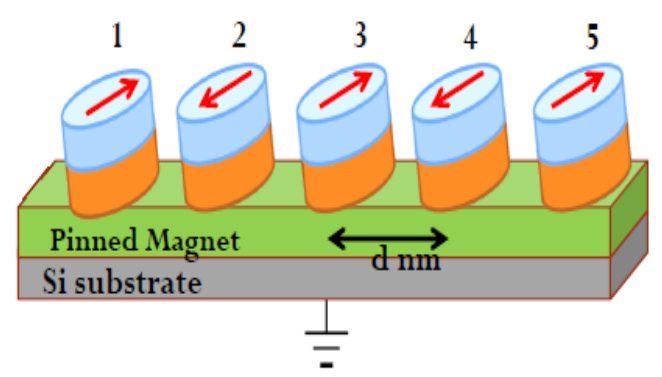

New information

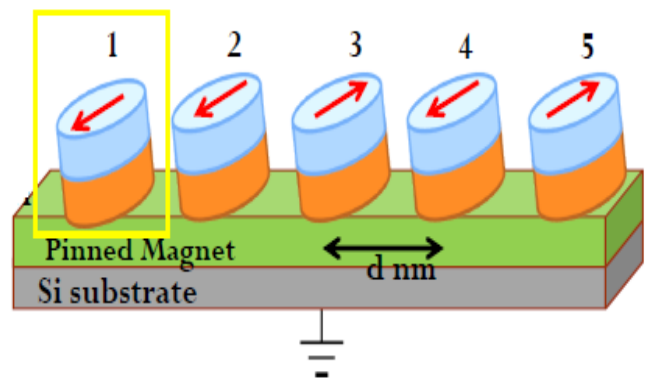

(d)

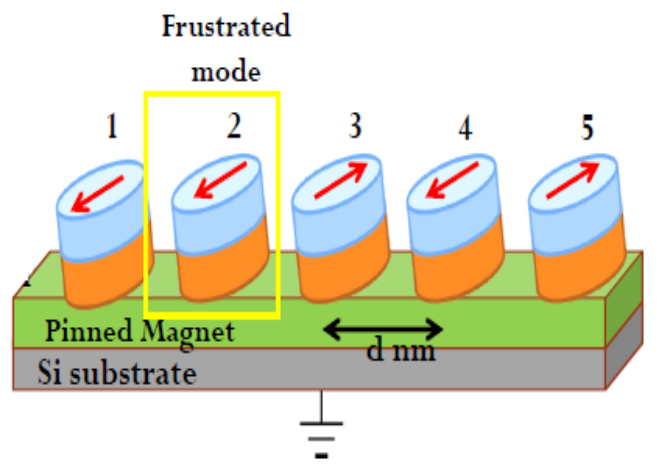

(e)

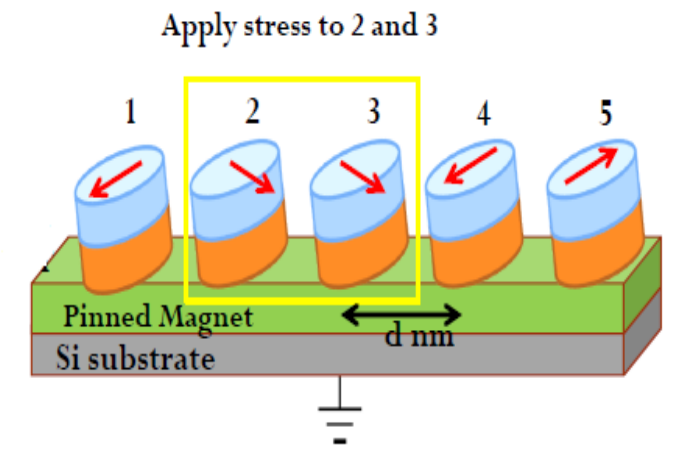

(f)

Release 2

(g)

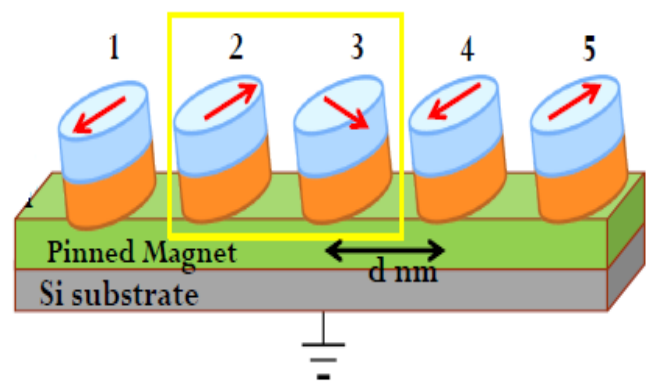

Apply stress to 4

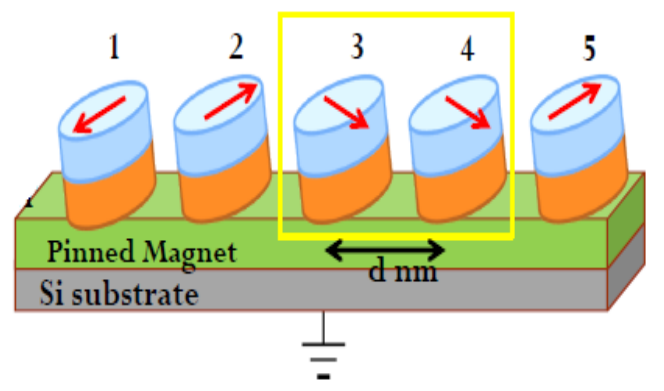

(h)

Release 3

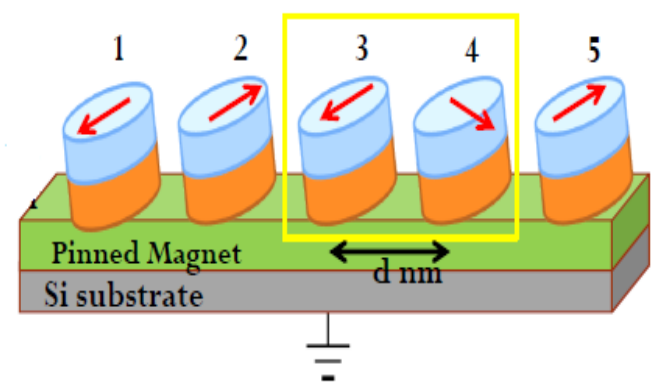

Figure 1.6: (a) In multiferroic logic, information is stored in the magnetostrictive Terfenol-D disk with an layer of piezoelectric below it. To Bennett-clock the disk, a voltage is applied to the PZT, causing is to stress. The stress is then converted to strain in the Terfenol-D, causing the magnetization to rotate. (b) Information is passed along an array of disks, which are coupled anti-ferromagnetically to each other in the ground state. (c)-(h) Information transmission from disk 1 to 3. 
respectively, per magnet at room temperature, if the clock period is $\sim 1 \mathrm{~ns}$.In multiferroic logic, the rotation is carried out by straining a multiferroic nanomagnet with a small voltage $(\sim 10 \mathrm{mV})$, consuming only an estimated $10^{2} K_{B} T$ per magnet [13]. Based on the dipole-coupled Bennett clocked MQCA architecture, an operating binary wire and majority gate have been designed and simulated at zero temperature ([21],[16]). An alternate MTJ architecture has also been proposed that does not rely on dipolar coupling, and is thus more scalable (dipolar couplings scale as the square of the volume) [22].

The specific design we will simulate here consists of a synthetic multiferroic stack with three layers - a magnetostrictive (Terfenol-D), a piezoelectric (PZT) and a hard magnetic layer (Fig. 1.6a). In the absence of any strain, the two mutually anti-parallel magnetizations along the major axis of the ellipse (easy axis of the Terfenol-D layer) encode the logic bits ' 0 ' and ' 1 ' ( $\phi=\pi / 2$ and $3 \pi / 2$ respectively) in either magnet [23]. The magnets are prepared for information propagation with a small local voltage applied to a coupled piezoelectric element (PZT), stressing the magnets and switching them onto their hard (in-plane minor) axes (Fig. 1.6a)[15].

Let us study how information is transmitted unidirectionally in a logic chain (or binary wire). In a chain of five magnets whose centers lie on a line parallel to the minor axis of the ellipses, the ground state is anti-ferromagnetic, meaning nearest neighbors have anti-parallel magnetizations (Fig. 1.6b). Thus, the logic bit hosted by the leftmost magnet is replicated in the rightmost magnet. If the leftmost magnet is flipped with an external agent (Fig. 1.6c), we expect the other two magnets to flip sequentially in a domino fashion for information propagation unidirectionally from left to right. However, this will not happen; the 2nd magnet will not flip since the dipole interactions from its two neighbors cancel (Fig. 1.6d). The 2nd magnet is said to be in frustrated mode. In order to make information propagate from left to right, Bennett clocking is implemented by stressing the 2 nd and 3 rd magnets to rotate their magnetizations by $\sim 90^{\circ}$ after the leftmost magnet has flipped (Fig. 1.6e). When stress is released on the 2nd magnet, it finds itself in a maximum energy state and subsequently relaxes to the minimum energy state by assuming an orientation anti-parallel to that of the magnet to its left because of the dipole coupling with its two neighbors (Fig. 1.6f). However, if the 2nd magnet fails to rotate under stress, then it may never flip since the combined dipole interactions from its two neighbors may not be able to make it transcend the energy barrier between its two stable magnetization states. To further transfer the information to the 3rd magnet, stress is applied to the 4th magnet (Fig. 1.6g) and 3 is released. Through dipole coupling with 2, 3's magnetization reaches the down spin state (Fig. 1.6h). 


\subsection{Challenges}

With its fast write and read, small cell size, non-volatility and excellent endurance, STT-RAM devices bear excellent potential of dominating the embedded and standalone memory world in the near future. However, in order to compete with existing embedded technology, an STT-RAM bit cell should be able to meet the following requirements [24]:

(i) TMR needs to exceed $150 \%$ for low-power read and adequately differentiate between bits " 1 " and " 0 ".

(ii) The thermal stability $\left(\Delta=H_{K} M_{S} / 2 K_{B} T\right.$ ) of the free layer must be greater than 60 to ensure enough stability against stochastic thermal switching to retain data for at least 10 years. $H_{K}$ is the anisotropy field while $M_{S}$ is the saturation magnetization.

(iii) A scalable spin transfer torque switching current $\left(\mathrm{J}<1.5 \times 10^{6} \mathrm{~A} / \mathrm{cm}^{2}\right)$.

(iv) A very low write-error-rate $(\mathrm{WER})<10^{-9}$ within a fast device write time $<10 \mathrm{~ns}$.

(v) An overall low energy write $(<1 \mathrm{pJ})$, meaning a correspondingly low switching voltage.

As the device holding the information is simple (a thin ferromagnetic layer), the performance parameters are related to each other. While the device desires high thermal stability, the critical current and the time needed to switch are also directly proportional to the thermal stability. Hence, greater thermal stability leads to a higher critical current. Higher thermal stability also contributes to longer switching tails, as the magnetization tends to be nearer to the stagnation point, $\theta=0$ or $\pi$, in the absence of an external perturbation. Near the stagnation point, the torque proportional to $\sin \theta$ is too small to initiate switching. Therefore a thermally stable free layer would require a relatively high current to have very low error rate $10^{-9}$ for switching within 5-10 ns. Achieving such a diverse set of targets will require meticulous design and material engineering.

The energy advantages of multiferroics have been discussed extensively in the literature, but several other issues that are important remain relatively unexplored. They include speed of operation, energy dissipation and error rates at room temperature. Till to date, most simulations of SML circuits have been carried out at zero temperature $[21,16]$ where thermal noise is absent and switching never fails. At room temperature, thermal noise generates random torques on the magnetization vector that can rotate the magnetization in 
the wrong direction and cause switching errors. For a single magnet used as a memory element, this can lead to write errors, but for a chain of dipole coupled magnets implementing a logic circuit, the effect is more serious since it can stop information propagation down the chain and cause widespread failure. Logic is less forgiving of errors than memory and calls for more stringent restrictions on error rates. Error rates in isolated magnets acting as memory elements has been studied before [25], but error rates in dipole coupled multiferroic magnets acting as logic chains have not been addressed. Logic computations should meet the following criteria:

(i) Highly reliable operation with error rate $\approx 10^{-15}-10^{-12}$.

(ii) Fast computation $<1 \mathrm{~ns}$.

\subsection{Aim of the dissertation}

The aim of the dissertation is to present a comprehensive simulation tool model for STT-RAM which is needed to explore the multi-parameter optimization problem involved (Chapter 2). Our current simulation tool uses the Non Equilibrium Green's Function (NEGF) method to solve quantum transport equations for the exerted torque, coupled with macrospin dynamics in the free layer, solved using the Landau-Lifshitz-Gilbert (LLG) equation in the presence of the both in-plane and in-field torque. The NEGF model works at three levels - (1) a parameterized model for quick, intuitive insights, (2) quasi-analytical physics based compact model, and (3) fully atomistic, chemistry based multi-orbital model for predictive material design and optimization. The LLG macromagnetic solver is a stochastic single domain model which can numerically solve the magnetization of the free layer in a STT-RAM, with both parallel and perpendicular anisotropy. The NEGF+ LLG comprehensive model specified is used to identify a set of materials that can meet the challenge of fast writing with low error threshold and energy consumption in single barrier STT-RAM (Chapter 3, 4 and 5).

A similar tool is presented for multiferroic logic that studies the error rate and switching delay. It is shown the performance of the logic computation can be controlled by material design and by engineering the stress profile on the nanomagnets. 


\section{Chapter 2}

\section{Unified model for STT-RAM}

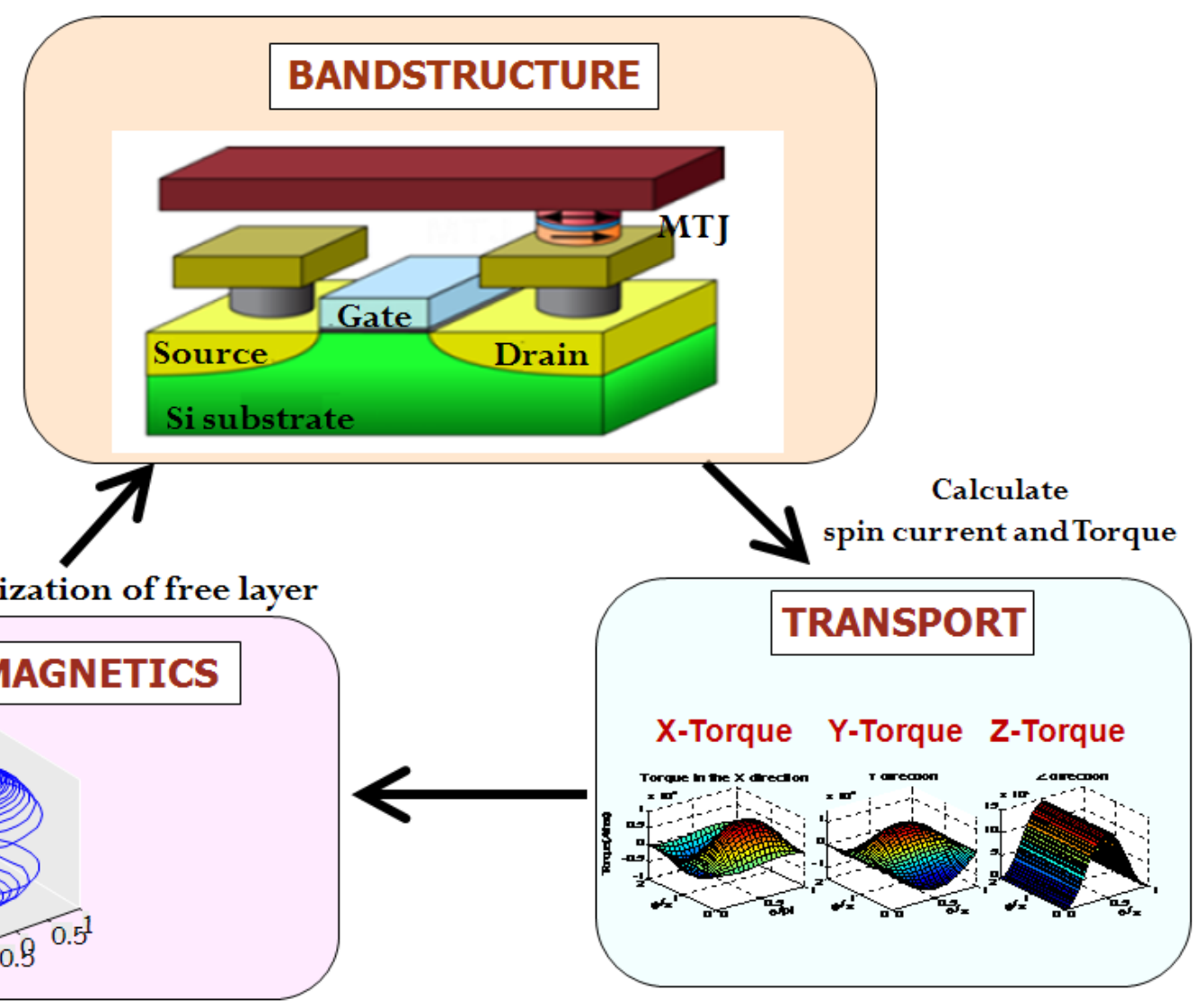

Figure 2.1: Fully integrated STT-RAM solver

Though spin-transport and spin-transfer torque in a magnetic tunnel [26, 27, 28, 29] and the macrospin dynamics $[30,31]$ of the of the free layer has been studied individually in great detail in the past, ours is the first comprehensive STT-RAM simulation tool to date (Fig. 2.1) that combines both the areas. The 
simulations are done self-consistently, to account for the continual change in interspin angle and torque during the evolution of the free spin. Given the description of the bandstructure of the magnetic tunnel junction, the transport module is used to calculated current and spin torque exerted on the free layer. The calculated spin torque is then fed into the stochastic macrodynamic module which can calculate the changing magnetization of the free layer. In this dissertation, we do not take into account the magnetic field generated in the junction because of current flow (BiotSavart).

\subsection{Bandstructure module}

The band structure of the MTJ can be specified in 3 ways: (2.1.1) a fully atomistic model, (2.1.2) a parameterized model using continuum grid, and (2.1.3) a quasi-analytical physics based compact model.

\subsubsection{Fully atomistic model}

In a fully atomistic description of the MTJ, detailed interfacial chemistry between the ferromagnetic bandstructure and the insulator and the correct density of states of the contacts are taken into account. The Hamiltonian of the tunnel junction can be calculated through various Density Functional Theory[32] packages available in the market today. For our study, we used two methods: Extended Huckel Theory (EHT) $[33,34,35]$, calibrated in turn with first principles Density Functional Theory (VASP[36]) and the combined effort of Siesta[37]+Smeagol[38, 39].
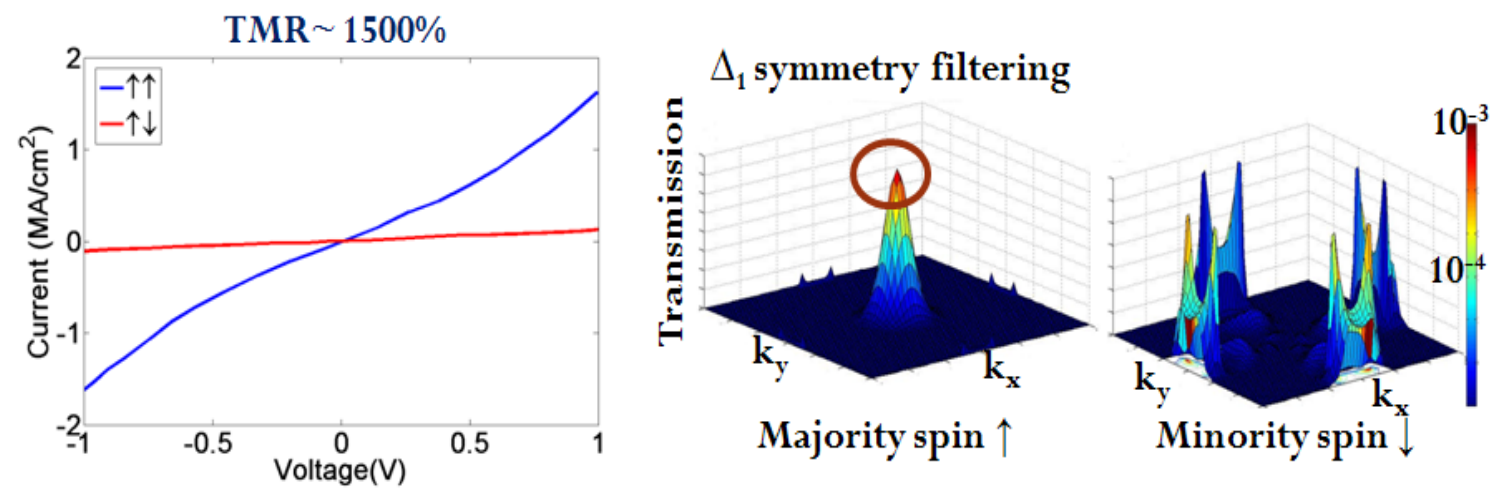

Figure 2.2: Atomistic Extended Huckel Theory + Quantum transport Strained Fe/MgO/Fe

The importance of the interfacial chemistry between the insulator and the ferromagnetic contacts is important in MTJs, as seen in the case of strained Fe/MgO/Fe (Fig. 2.2). Even though bulk iron is not a halfmetallic in nature, with addition to a $\mathrm{MgO}$ barrier, it has half metallic tunneling characteristics[40, 41, 42, 43]. This calculation has been done by EHT parameters extracted by Professor William Butler's group in University 
(a)

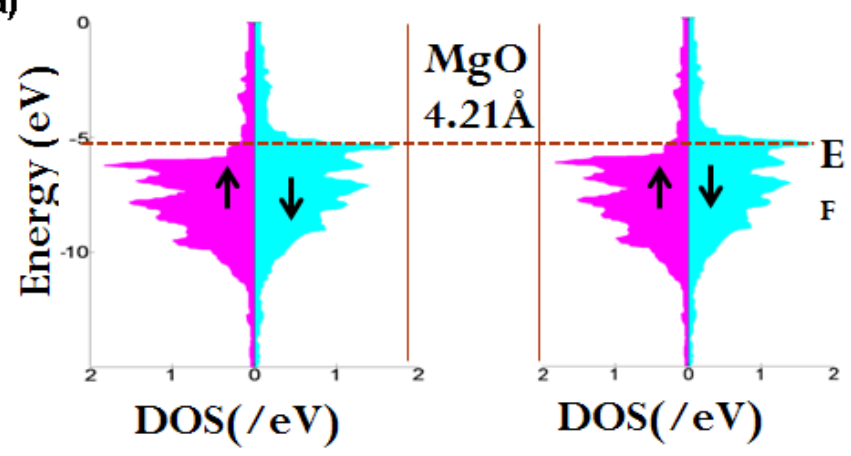

(b)

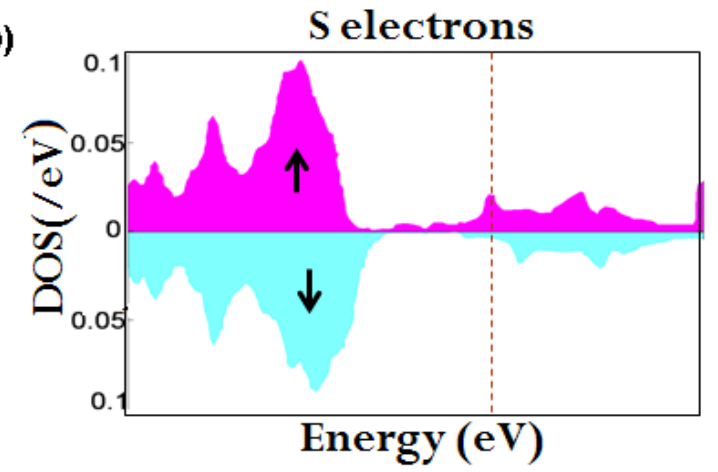

(c)

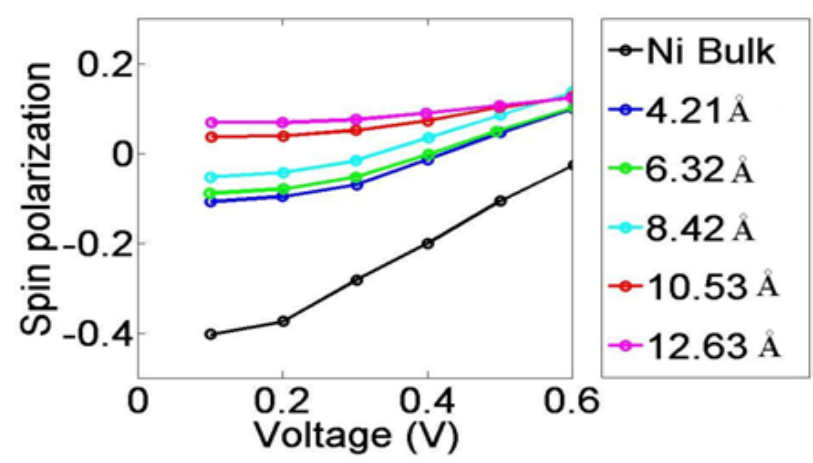

Figure 2.3: Mass dependent tunneling in $\mathrm{Ni} / \mathrm{MgO} / \mathrm{Ni}$ MTJ

of Alabama through VASP. At the [001] interface of $\mathrm{MgO}$, the $\Delta_{1} \mathrm{~s}$ valence bands continues as an evanescent state into the band gap and then becomes the conduction band. Electrons tunneling through this evanescent states will have a much slower decay rate than tunneling through an insulation gap. Iron has a s $\Delta_{1}$ up spin band near its Fermi level, enabling the electrons to tunnel through the evanescent states. However, the down spin $\Delta_{2}$ and $\Delta_{5}$ bands will see a barrier. Therefore in parallel configuration, the s up spin electrons will decay slowly through the tunnel while the $\Delta_{2}$ and $\Delta_{5} \mathrm{~d}$ down spin band electrons will decay faster. There will be removal of $\Delta_{2}$ and $\Delta_{5} \mathrm{~d}$ down bands by chemistry and amplification of $\Delta_{1} \mathrm{~s}$ up bands by Fermi level placement with the $\mathrm{MgO}$ evanescent state.

As seen in the transmission plot across the Brillouin zone, transmission for up spin is $10^{-3}$ while for minority is $10^{-4}$. At anti-parallel configuration, the preferential tunneling will not exist, so that results in a very high TMR in Fe/MgO/Fe MTJ, around 1500\% near zero voltage. The DFT prediction of the preferential tunneling through $\mathrm{MgO}$ insulator has lead to quite a revolution in the experimental world and industry. Previously, the most common insulator used in a MTJ was amorphous aluminum oxide which resulted in low TMR. Switching to MgO has led to higher TMR, as indicated in this experimental result, $25 \%$ vs $150 \%[44]$. The experimental TMR is lower compared that predicted by theory because of manufacturing defects $[45,46]$

Doing fully atomistic study will help uncover interesting physics as seen in the mass dependent tunneling 


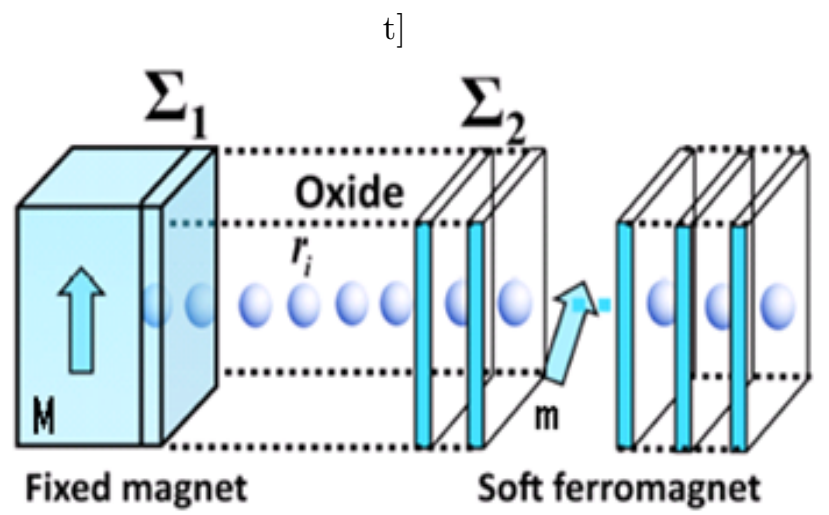

Figure 2.4: Continuum grid representation of a MTJ

in $\mathrm{Ni} / \mathrm{MgO} / \mathrm{Ni}$ tunnel junction using the package Siesta+Smeagol (Fig. 2.3). For a very narrow $\mathrm{MgO}$ barrier, the tunneling current is dominated by the down spin electrons in the $\mathrm{Ni} \mathrm{d}$ bands as there are more down electrons than up electrons. The spin polarization of the tunneling current can be defined as,

$$
\text { Spinpolarization }=\frac{I_{\uparrow}-I_{\downarrow}}{I_{\uparrow}+I_{\downarrow}}
$$

For a narrow $\mathrm{MgO}$ barrier, we would get a negative spin polarized current (Fig 2.3c). Now the interesting physics happen as you increase the $\mathrm{MgO}$ width. Spin polarized current becomes positive, that means we are getting more up spin current than down spin current. D electron mass is heavier than s electron mass and we know the tunneling transmission is proportional to exponential of the mass, $T \approx \exp (\sqrt{m})$. At lower width, d down electrons could dominate because they were greater in number, but at wider width, they are too heavy to tunnel through and the s electrons take over. As there is more s up electrons than s down electrons, you get positive spin polarized current.

\subsubsection{Parameterized continuum grid model}

The continuum grid Hamiltonian $(\mathrm{H})($ Fig 2.4$)$ and the spin-dependent contacts self-energy matrices $\left(\Sigma_{L, R}\right)$ for the single barrier MTJ in a STT-RAM can be specified by the following five parameters[47]: (i) $U$ is the barrier offset between the contact and the insulator, (ii) $E_{F}$ is Fermi-level,(iii) $\delta$ is the band splitting between majority and minority spin electrons, (iV) $\mathrm{W}$ is the width of the insulator, (v) $m_{c}$ and (vi) $m_{\text {barr }}$ are the effective masses in the contact and barrier respectively ( Fig. 2.5). Further explanation on how to set up this model is given in Appendix A. 


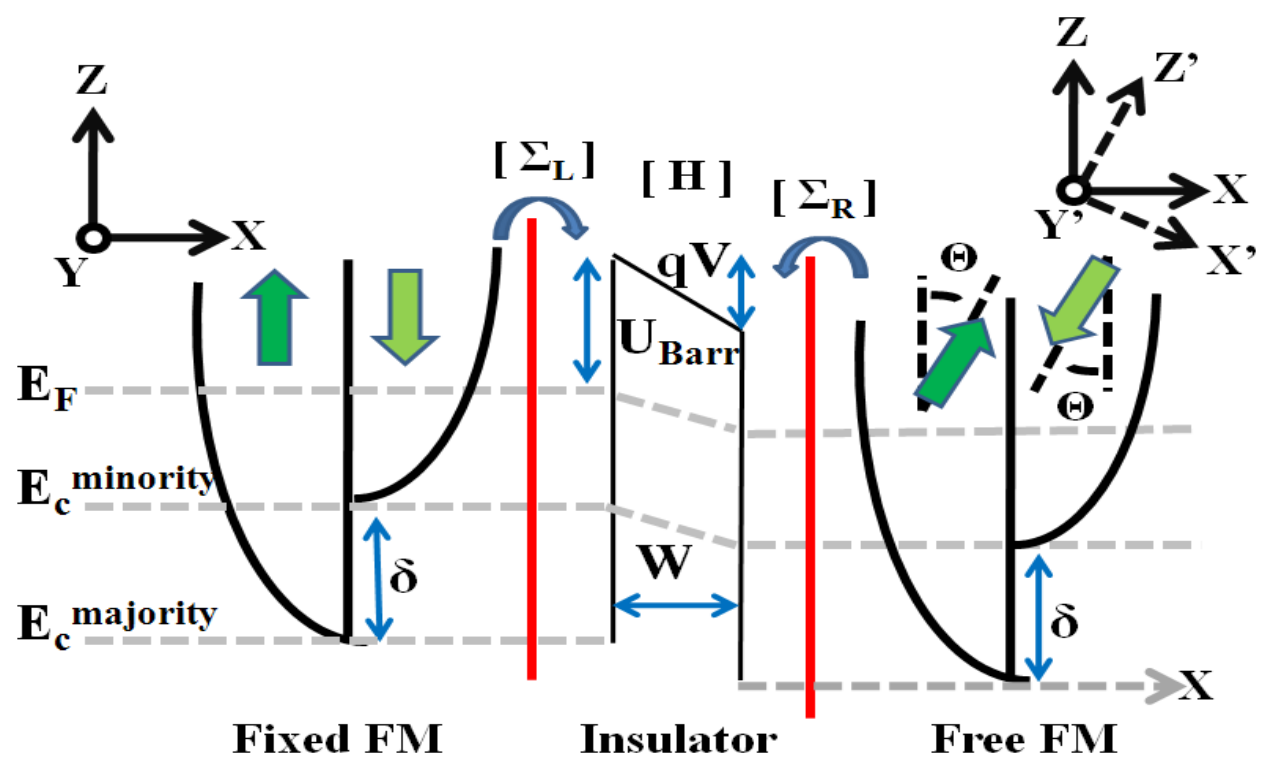

Figure 2.5: Band structure of a magnetic tunnel junction. The bottom of $\uparrow$ and $\downarrow$ conduction bands in ferromagnetic $(\mathrm{FM})$ contacts are separated by $\delta \mathrm{eV}$, while the insulating oxide introduces a barrier. $E_{F}$ is the Fermi level, $\mathrm{W}$ is the width of the insulating barrier and $\mathrm{U}$ is is the barrier offset between the contact and the insulator. The magnetization of the right layer is fixed, while the left layer is free to rotate.

\subsubsection{Quasi-analytical physics based compact model}

In the quasi-analytical physics based compact model, the same set of parameters using in the parameterized continuum grid model is used to describe the Hamiltonian of the magnetic tunnel junction (Fig. 2.5).

\subsection{Transport module}

The spin torque exerted on the free magnetic layer can be calculated at two level of complexities (2.2.1) Non-Equilibrium Green's Function (NEGF) for the atomistic and parameterized continuum grid bandstructure and 2.2.2) Modified Simmons' equation for the quasi-analytical physics based compact model.

\subsubsection{Non-Equilibrium Green's Function}

The NEGF formalism is employed to calculate the ballistic tunneling current and spin current from lattice site $\mathrm{j}$ to $\mathrm{j}+1$ from Green's function $(\mathrm{G})$ and electron correlation function $\left(G^{n}\right)[48]$ : 


\section{Green's function:}

$$
G(E)=\left(E I-H-\Sigma_{L}(E)-\Sigma_{R}(E)\right)^{-1}
$$

\section{Electron correlation function:}

$$
G^{n}(E)=G(E)\left(\Sigma_{L}^{i n}(E)+\Sigma_{R}^{i n}(E)\right) G(E)^{\dagger}
$$

\section{In-scattering function:}

$$
\Sigma_{L, R}(E)^{i n}=\gamma_{L, R}(E) F_{L, R}(E)
$$

Broadening Matrix:

$$
\gamma_{L, R}(E)=i\left(\Sigma_{L, R}(E)-\Sigma_{L, R}(E)^{\dagger}\right)
$$

\section{Current:}

$I_{j, j \pm 1}=\frac{1}{i \hbar} \int d E\left[\operatorname{Tr}\left(H_{j, j \pm 1} G_{j \pm 1, j}^{n}-G_{j, j \pm 1}^{n} H_{j \pm 1, j}\right)\right]$

\section{Spin current:}

$$
I_{j, j \pm 1}^{S}=\frac{1}{i \hbar} \int d E\left[\operatorname{Tr}\left(\vec{\sigma}_{S}\left(H_{j, j \pm 1} G_{j \pm 1, j}^{n}-G_{j, j \pm 1}^{n} H_{j \pm 1, j}\right)\right)\right]
$$

where $\vec{\sigma}_{S}$ are the Pauli spin matrices and $F_{L, R}(E)$ are the 2-D Fermi function at the two contacts. Using the 2-D Fermi function takes care of the transverse momentum in the $\mathrm{Y}$ and $\mathrm{Z}$ directions. The spin torque exerted on the free magnetic layer will be the spin current generated at the insulator - free layer interface and absorbed by the free layer. The absorbed voltage dependent torque has the general structure

$$
\vec{T}(V)=\left[A(V) \hat{m} \times\left(\hat{m} \times \hat{m}_{p}\right)+B(V) \hat{m} \times \hat{m}_{p}\right]
$$

where the first is the current driven spin-transfer torque, while the second is the field-like torque arising from direct exchange coupling between the two ferromagnets. $\hat{m}$ and $\hat{m}_{p}$ represent the unit vectors for the magnetizations in the free and pinned layers respectively.

\subsubsection{Modified Simmons' model}

The magnetic tunnel junction in a STT-RAM can be broken up into three regions (Fig 1$):(\mathrm{I}) X<0$ - the left fixed ferromagnetic layer where the magnetization of the left layer is pinned to the $+\mathrm{Z}$ axis, (II) $0 \leqslant X \leqslant W$ the insulating tunneling barrier and (III) $X>W$-the right ferromagnetic layer whose magnetization is free to rotate and is defined by the angle $\theta$ measured with respect to the positive $+\mathrm{Z}$ axis (Fig. 2.5). The magnetization of the right ferromagnet is parallel to Z' axis of the coordinate system X', Y',Z, which is rotated $\theta$ degree to the original $\mathrm{Z}$ axis. For simplicity, we are omitting the transverse momentum $k_{\|}$and assuming 
that potential barrier in region II is rectangular when voltage is applied. In a free electron approximation, the longitudinal spin-polarized electron momentum in each of the three region can be expressed as

Region I, fixed contact: $k_{\uparrow}=\frac{\sqrt{2 m E}}{\hbar}, k_{\downarrow}=\frac{\sqrt{2 m(E-\delta)}}{\hbar}$

Region II, insulator: $\kappa=\frac{\sqrt{2 m(U-E)}}{\hbar}$

Region III, free contact: $k_{\uparrow}^{+}=\frac{\sqrt{2 m(E-q V)}}{\hbar}, k_{\downarrow}^{+}=\frac{\sqrt{2 m(E-\delta-q V)}}{\hbar}$

where $\mathrm{m}$ is the mass of electron, $\hbar$ is Plank's constant, $\mathrm{U}$ is the barrier offset inside the insulator and $\delta$ is the band splitting between majority and minority spin bands. The right contact has an additional -qV potential drop because of an applied positive voltage. Positive voltage is applied to the MTJ to switch from anti-parallel to parallel configuration. Considering a spin-up incident plane wave in Region I, the wave functions in the three regions are:

$$
\begin{aligned}
\text { Region I: } \psi_{I \uparrow} & =\frac{1}{\sqrt{k_{\uparrow}}} e^{i k_{\uparrow} x}+R_{\uparrow} e^{-i k_{\uparrow} x} \\
\psi_{I \downarrow} & =R_{\downarrow} e^{-i k_{\downarrow} x} \\
\text { Region II: } \psi_{I I \uparrow} & =A_{\uparrow} e^{-\kappa x}+B_{\uparrow} e^{\kappa x} \\
\psi_{I I \downarrow} & =A_{\downarrow} e^{-\kappa x}+B_{\downarrow} e^{\kappa x} \\
\text { Region III: } \psi_{I I I \uparrow}^{\prime} & =C_{\uparrow} e^{i k_{\uparrow}^{+} x} \\
\psi_{I I I \downarrow}^{\prime} & =C_{\downarrow} e^{i k_{\downarrow}^{+} x}
\end{aligned}
$$

The wave functions in the third region, $\psi_{I I I \uparrow}^{\prime}$ and $\psi_{I I I \downarrow}^{\prime}$, are with respect to the rotated axes, $\mathrm{X}^{\prime}, \mathrm{Y}^{\prime}, \mathrm{Z}^{\prime}$. In order to conform to the original axes, a spinor transformation is required[28],

$$
\begin{gathered}
\psi_{I I I \uparrow}=\cos \left(\frac{\theta}{2}\right) \psi_{I I I \uparrow}^{\prime}+\sin \left(\frac{\theta}{2}\right) \psi_{I I I \downarrow}^{\prime} \\
\psi_{I I I \downarrow}=-\sin \left(\frac{\theta}{2}\right) \psi_{I I I \uparrow}^{\prime}+\cos \left(\frac{\theta}{2}\right) \psi_{I I I \downarrow}^{\prime}
\end{gathered}
$$

By matching $\psi_{\uparrow, \downarrow}$ and $d \psi_{\uparrow, \downarrow} / d x$ at $\mathrm{x}=0$ and $\mathrm{W}$, the unknowns, $R_{\uparrow, \downarrow}$ and $C_{\uparrow, \downarrow}$ are solved to the leading order of $e^{-\kappa w}$, which can be used to calculate the torque inducing traverse spin current in Region I and III. 


$$
\begin{gathered}
C_{\uparrow}=\frac{-4 \kappa \sqrt{k_{\uparrow}} \cos \left(\frac{\theta}{2}\right) e^{-\kappa w}}{\left(\kappa-i k_{\uparrow}\right)\left(\kappa-i k_{\uparrow}^{+}\right)} \\
C_{\downarrow}=\frac{-4 \kappa \sqrt{k_{\uparrow}} \sin \left(\frac{\theta}{2}\right) e^{-\kappa w}}{\left(\kappa-i k_{\uparrow}\right)\left(\kappa-i k_{\downarrow}^{+}\right)} \\
R_{\uparrow}=\frac{\kappa+i k_{\uparrow}}{\kappa-i k_{\uparrow}} \\
R_{\downarrow}=\frac{-4 i \kappa \sqrt{k_{\uparrow}} \kappa^{2}\left(k_{\uparrow}^{+}-k_{\downarrow}^{+}\right) \sin (\theta) e^{-2 \kappa w}}{\left(\kappa-i k_{\uparrow}\right)\left(\kappa-i k_{\downarrow}\right)\left(\kappa-i k_{\uparrow}^{+}\right)\left(\kappa-i k_{\downarrow}^{+}\right)}
\end{gathered}
$$

The charge current and spin currents in Region I and III are calculated by,

$$
\begin{gathered}
J=\frac{q \hbar}{2 m i}\left[\left(\begin{array}{ll}
\psi_{\uparrow}^{*} & \psi_{\downarrow}^{*}
\end{array}\right)\left(\begin{array}{c}
\frac{d \psi_{\uparrow}}{d x} \\
\frac{d \psi_{\downarrow}}{d x}
\end{array}\right)-\left(\begin{array}{ll}
\psi_{\uparrow} & \psi_{\downarrow}
\end{array}\right)\left(\begin{array}{c}
\frac{d \psi_{\uparrow}^{*}}{d x} \\
\frac{d \psi_{\downarrow}^{*}}{d x}
\end{array}\right)\right] \\
J_{\sigma}=\frac{q \hbar}{2 m i}\left[\left(\begin{array}{ll}
\psi_{\uparrow}^{*} & \psi_{\downarrow}^{*}
\end{array}\right) \sigma\left(\begin{array}{c}
\frac{d \psi_{\uparrow}}{d x} \\
\frac{d \psi_{\downarrow}}{d x}
\end{array}\right)-\left(\begin{array}{ll}
\psi_{\uparrow} & \psi_{\downarrow}
\end{array}\right) \sigma\left(\begin{array}{c}
\frac{d \psi_{\uparrow}^{*}}{d x} \\
\frac{d \psi_{\downarrow}^{*}}{d x}
\end{array}\right)\right]
\end{gathered}
$$

where $\sigma$ are the Pauli matrices. The spin current component parallel to the magnetization of the ferromagnet in the third region, $J_{I I I, Z}$, tunnel through and has no contribution to the torque. The transverse spin currents, $J_{I I I, X}$ and $J_{I I I, Y}$ gets absorbed by the ferromagnet in the third region which induces the torque that acts on the magnetization.

$$
\begin{gathered}
J_{I I I, \uparrow}=\frac{16 k_{\uparrow} \kappa^{2} q \hbar e^{-2 \kappa w}}{m\left(\kappa^{2}+k_{\uparrow}^{2}\right)}\left[\frac{k_{\uparrow}^{+} \cos ^{2}\left(\frac{\theta}{2}\right)}{\left(\kappa^{2}-k_{\uparrow}^{+2}\right)}+\frac{k_{\downarrow}^{+} \sin ^{2}\left(\frac{\theta}{2}\right)}{\left(\kappa^{2}+k_{\downarrow}^{+2}\right)}\right] \\
J_{I I I, Z, \uparrow}=\frac{16 k_{\uparrow} \kappa^{2} q \hbar e^{-2 \kappa w}}{m\left(\kappa^{2}+k_{\uparrow}^{2}\right)}\left[\frac{k_{\uparrow}^{+} \cos ^{2}\left(\frac{\theta}{2}\right)}{\left(\kappa^{2}-k_{\uparrow}^{+2}\right)}-\frac{k_{\downarrow}^{+} \sin ^{2}\left(\frac{\theta}{2}\right)}{\left(\kappa^{2}+k_{\downarrow}^{+2}\right)}\right] \\
J_{I I I, X, \uparrow}=\frac{8 k_{\uparrow} \kappa^{2} q \hbar e^{-2 \kappa w}\left(k_{\uparrow}^{+}+k_{\downarrow}^{+}\right) \sin (\theta)}{m\left(\kappa^{2}+k_{\uparrow}^{2}\right)\left(\kappa^{2}+k_{\uparrow}^{+2}\right)\left(\kappa^{2}+k_{\downarrow}^{+2}\right)} X_{I I I} \\
J_{I I I, Y, \uparrow}=\frac{8 i k_{\uparrow}^{2} \kappa^{2} q \hbar e^{-2 \kappa w}\left(k_{\uparrow}^{+}-k_{\downarrow}^{+}\right) \sin (\theta)}{m\left(\kappa^{2}+k_{\uparrow}^{2}\right)\left(\kappa^{2}+k_{\uparrow}^{+2}\right)\left(\kappa^{2}+k_{\downarrow}^{+2}\right)} X_{I I I} \\
X_{I I I}=\left[\left(\kappa^{2}+k_{\uparrow}^{+} k_{\downarrow}^{+}\right) \cos \left(k_{\uparrow}^{+} x-k_{\downarrow}^{+} x\right)+\kappa\left(k_{\uparrow}^{+}-k_{\downarrow}^{+}\right) \sin \left(k_{\uparrow}^{+} x-k_{\downarrow}^{+} x\right)\right]
\end{gathered}
$$


Similarly for a incoming down spin electron, the unknowns, $R_{\uparrow, \downarrow}$ and $C_{\uparrow, \downarrow}$ are solved to the leading order of $e^{-\kappa w}$,

$$
\begin{gathered}
C_{\uparrow}=\frac{4 \kappa \sqrt{k_{\downarrow}} \sin \left(\frac{\theta}{2}\right) e^{-\kappa w}}{\left(\kappa-i k_{\downarrow}\right)\left(\kappa-i k_{\uparrow}^{+}\right)} \\
C_{\downarrow}=\frac{-4 \kappa \sqrt{k_{\downarrow}} \cos \left(\frac{\theta}{2}\right) e^{-\kappa w}}{\left(\kappa-i k_{\downarrow}\right)\left(\kappa-i k_{\downarrow}^{+}\right)} \\
R_{\uparrow}=\frac{-4 i \kappa=\sqrt{k_{\downarrow}} \kappa^{2}\left(k_{\uparrow}^{+}-k_{\downarrow}^{+}\right) \sin (\theta) e^{-2 \kappa w}}{\left(\kappa-i k_{\uparrow}\right)\left(\kappa-i k_{\downarrow}\right)\left(\kappa-i k_{\uparrow}^{+}\right)\left(\kappa-i k_{\downarrow}^{+}\right)} \\
R_{\downarrow}=\frac{\kappa+i k_{\downarrow}}{\kappa-i k_{\downarrow}}
\end{gathered}
$$

The corresponding spin currents,

$$
\begin{aligned}
& J_{I I I, X, \downarrow}=-\frac{8 k_{\downarrow} \kappa^{2} q \hbar e^{-2 \kappa w}\left(k_{\uparrow}^{+}+k_{\downarrow}^{+}\right) \sin (\theta)}{m\left(\kappa^{2}+k_{\downarrow}^{2}\right)\left(\kappa^{2}+k_{\uparrow}^{+2}\right)\left(\kappa^{2}+k_{\downarrow}^{+2}\right)} X_{I I I} \\
& J_{I I I, Y, \downarrow}=-\frac{8 i k_{\downarrow} \kappa^{2} q \hbar e^{-2 \kappa w}\left(k_{\uparrow}^{+}-k_{\downarrow}^{+}\right) \sin (\theta)}{m\left(\kappa^{2}+k_{\downarrow}^{2}\right)\left(\kappa^{2}+k_{\uparrow}^{+2}\right)\left(\kappa^{2}+k_{\downarrow}^{+2}\right)} X_{I I I}
\end{aligned}
$$

Adding both torque exerted by up and down spin electrons,we see that the total torque is proportional to polarization of the first contact,

$$
J_{I I I, X, \text { total }}, J_{I I I, Y, \text { total }} \propto \frac{k_{\uparrow}-k_{\downarrow}}{\left(\kappa^{2}+k_{\uparrow}^{2}\right)\left(\kappa^{2}+k_{\downarrow}^{2}\right)}
$$

The intensity of the torque will increase with increasing polarization of the contact.

The first region also experience torque inducing traverse currents where angular momentum is removed. This torque is analogous to the the torque used in parallel to anti-parallel switching when a negative voltage is applied.

$$
\begin{gathered}
J_{I, Z}=\frac{-8 q \hbar \kappa^{4} k_{\uparrow}^{2} k_{\downarrow}\left(k_{\uparrow}^{+}-k_{\downarrow}^{+}\right) e^{-4 \kappa w} \sin ^{2}(\theta)}{\left(\kappa-i k_{\uparrow}^{2}\right)\left(\kappa-i k_{\downarrow}^{2}\right)\left(\kappa-i k_{\uparrow}^{+2}\right)\left(\kappa-i k_{\downarrow}^{+2}\right)} \\
J_{I, X}=\frac{-4 q \hbar \kappa^{2} k_{\uparrow}\left(k_{\uparrow}^{+}-k_{\downarrow}^{+}\right) e^{-2 \kappa w} \sin (\theta)}{\left(\kappa-i k_{\uparrow}^{2}\right)\left(\kappa-i k_{\downarrow}^{2}\right)\left(\kappa-i k_{\uparrow}^{+2}\right)\left(\kappa-i k_{\downarrow}^{+2}\right)} X_{I} \\
J_{I, Y}=\frac{4 i q \hbar \kappa^{2} k_{\uparrow}\left(k_{\uparrow}^{+}-k_{\downarrow}^{+}\right) e^{-2 \kappa w} \sin (\theta)}{\left(\kappa-i k_{\uparrow}^{2}\right)\left(\kappa-i k_{\downarrow}^{2}\right)\left(\kappa-i k_{\uparrow}^{+2}\right)\left(\kappa-i k_{\downarrow}^{+2}\right)} X_{I}
\end{gathered}
$$




$$
\begin{aligned}
& X_{I}= {\left[( k _ { \uparrow } - k _ { \downarrow } ) \left(E \cos \left(k_{\uparrow} x+k_{\downarrow} x\right)-F \sin \left(k_{\uparrow} x+k_{\downarrow} x\right)\right.\right.} \\
&-\left(k_{\uparrow}-k_{\downarrow}\right)\left(H \cos \left(k_{\uparrow} x-k_{\downarrow} x\right)-I \sin \left(k_{\uparrow} x-k_{\downarrow} x\right)\right]
\end{aligned}
$$

where,

$$
\begin{aligned}
& E=\kappa^{4}-\kappa^{2} k_{\uparrow} k_{\downarrow}-\kappa^{2} k_{\uparrow}^{+} k_{\downarrow}^{+}-\kappa^{2} k_{\downarrow} k_{\downarrow}^{+}-\kappa^{2} k_{\downarrow} k_{\uparrow}^{+}-\kappa^{2} k_{\uparrow} k_{\downarrow}^{+}-\kappa^{2} k_{\uparrow} k_{\uparrow}^{+}+k_{\uparrow} k_{\downarrow} k_{\uparrow}^{+} k_{\downarrow}^{+} \\
& F=-\kappa^{3} k_{\uparrow}-\kappa^{3} k_{\downarrow}-\kappa^{3} k_{\uparrow}^{+}-\kappa^{3} k_{\downarrow}^{+}+\kappa k_{\uparrow} k_{\uparrow}^{+} k_{\downarrow}^{+}+\kappa k_{\downarrow} k_{\uparrow}^{+} k_{\downarrow}^{+}+\kappa k_{\uparrow} k_{\downarrow} k_{\uparrow}^{+}+\kappa k_{\downarrow} k_{\downarrow} k_{\downarrow}^{+} \\
& H=\kappa^{4}+\kappa^{2} k_{\uparrow} k_{\downarrow}-\kappa^{2} k_{\uparrow}^{+} k_{\downarrow}^{+}-\kappa^{2} k_{\downarrow} k_{\downarrow}^{+}-\kappa^{2} k_{\downarrow} k_{\uparrow}^{+}+\kappa^{2} k_{\uparrow} k_{\downarrow}^{+}+\kappa^{2} k_{\uparrow} k_{\uparrow}^{+}-k_{\uparrow} k_{\downarrow} k_{\uparrow}^{+} k_{\downarrow}^{+} \\
& I=\kappa^{3} k_{\uparrow}-\kappa^{3} k_{\downarrow}-\kappa^{3} k_{\uparrow}^{+}-\kappa^{3} k_{\downarrow}^{+}-\kappa k_{\uparrow} k_{\uparrow}^{+} k_{\downarrow}^{+}+\kappa k_{\downarrow} k_{\uparrow}^{+} k_{\downarrow}^{+}-\kappa k_{\uparrow} k_{\downarrow} k_{\uparrow}^{+}-\kappa k_{\downarrow} k_{\downarrow} k_{\downarrow}^{+}
\end{aligned}
$$

While the torque in the right contact adds angular momentum to induce anti-parallel to parallel switching, there is a reciprocal torque in the left contact that removes angular momentum and induces parallel to anti-parallel switching. The torque at the left layer is dependent on the polarization of the left layer, $k_{\uparrow}^{+}-k_{\downarrow}^{+}$.

Therefore, by using the methodology in [28], a weighted sum of the tunneling up and down spin electrons was obtained, with weighting factors given by their respective densities of states. The transverse modes with momentum $k_{\|}$, and a linear potential drop in the insulator were included using the WKB approximation prescribed by Simmons in [49]. The total current density, J, at a given voltage and angle $\theta$ in a magnetic tunnel junction is,

$$
\begin{gathered}
J_{0}(V)=\frac{q^{2}}{4 \pi^{2} \hbar W^{2}}\left[(U-q V / 2) e^{\left.-\frac{2 W}{\hbar} \sqrt{2 m_{c}(U-q V / 2}\right)}-(U+q V / 2) e^{\left.-\frac{2 W}{\hbar} \sqrt{2 m_{c}(U+q V / 2)}\right]}\right. \\
J_{\sigma}=\frac{16 k_{\sigma} \beta^{2}}{\beta^{2}+k_{\sigma}^{2}}\left[\frac{k_{\sigma}^{+} \cos ^{2}(\theta / 2)}{\beta^{2}+k_{\sigma}^{+2}}+\frac{k_{\bar{\sigma}}^{+} \sin ^{2}(\theta / 2)}{\beta^{2}+k_{\bar{\sigma}}^{+2}}\right], \begin{array}{l}
\sigma=\uparrow, \downarrow \\
\bar{\sigma}=\downarrow, \uparrow
\end{array} \\
J(V, \theta)=\left[J_{\uparrow}(V, \theta)+J_{\downarrow}(V, \theta)\right] J_{0}(V)
\end{gathered}
$$

From the spin current, we can calculate the torque exerted on the free magnetic layer [50], $\vec{T}(V)=$ $(\hbar / 2 q) J_{\text {avg }}(V) \eta(V)\left[\hat{m} \times\left(\hat{m} \times \hat{m}_{p}\right)\right]$ where $J_{\text {avg }}(V)=[J(V, \theta=0)+J(V, \theta=\pi)] / 2 . \hat{m}$ and $\hat{m}_{p}$ represent the unit vector orientations for the magnetizations in the free and fixed layers respectively. When a positive bias is applied, polarized electrons flow from the fixed FM contact to the free layer, causing the magnetization of the free layer to switch from anti-parallel to parallel. For negative bias, the electron flow removes magnetization from the right layer, causing it to rotate from P to AP. Notice that the torque exerted at the right, free ferromagnetic layer depends on the effective voltage-dependent polarization of the incident electrons from the 
left, fixed layer[51]. This voltage dependence is critical to understanding the observed $\mathrm{P}$ to AP vs AP to P asymmetry in STTRAMs. The effective polarization of the incident electrons at a given voltage is given by,

$$
\eta(V)=\frac{k_{\uparrow}-k_{\downarrow}}{k_{\uparrow}+k_{\downarrow}}
$$

\subsection{Stochastic macrospin dynamics}

Finally, the calculated torque from the transport module is included in a modified Landau-Lifschitz-Gilbert (LLG) equation for the magnetization dynamics in the free layer,

$$
\frac{d \hat{m}}{d t}-\alpha\left(\hat{m} \times \frac{d \hat{m}}{d t}\right)=-\gamma \hat{m} \times \vec{H}_{e f f}+\vec{T}(V)
$$

where $\alpha$ is the damping coefficient, $\gamma$ is the gyro-magnetic ratio and $\vec{H}_{e f f}$ is the effective magnetic field that includes the effects of magnetocrystalline and shape anisotropy as well as the influence of external magnetic fields. The magnetocrystalline anisotropy is modeled by $E=K \sin ^{2}(\theta)$, where $\mathrm{K}$ in the intrinsic anisotropy constant for the ferromagnet. The shape anisotropy is dependent on the orientation of the magnetization of the elliptical ferromagnetic disk. If the magnetization of the free layer ferromagnet lies within the plane of the elliptical disk (in-plane ferromagnetic contact), the shape anisotropy is $E=4 \pi M_{S}^{2} \sin ^{2}(\theta) \cos ^{2}(\phi)$ where $M_{S}$ is the saturation magnetization[52]. If the magnetization lies perpendicular to the disk, the shape anisotropy is $E=4 \pi M_{S}^{2} \cos ^{2}(\theta)$.

The thermal perturbation is included by a Langevin random field $\vec{H}_{L}$, that can be added to the effective magnetic field term. The field $\vec{H}_{L}$ relates to the system temperature T by,

$$
\vec{H}_{L}=\sqrt{\frac{2 \alpha K_{B} T}{\gamma \Omega M_{s}}} G
$$

where $\mathrm{G}$ is a Gaussian white noise with mean 0 and standard deviation of 1 .

An alternate to the stochastic approach is to solve the Fokker-Planck equation for the probability density of each configuration and then average over the distribution to extract various moments [53].

\subsection{Benchmarking with Experiments}

The unified model is benchmarked with a published experiment to test for accuracy. The NEGF model was used to fit the resistance VS voltage data for in-plane rectangular $70 \mathrm{~nm} \times 250 \mathrm{~nm} \mathrm{CoFeB} / \mathrm{MgO} / \mathrm{CoFeB}(2 \mathrm{~nm})$ (Fig. 2.6a and b) [54]. The parameters extracted from the model are $E_{F}=2.25 \mathrm{eV}, \mathrm{U}=1 \mathrm{eV}$, width $=1 \mathrm{~nm}$, 
$m_{c}=1.3, m_{\text {barr }}=0.32$ and $\delta=1.96 \mathrm{eV}$. The parameters were then used to calculate torque that served as an input into the LLG solver. In the experiment, AP to $\mathrm{P}$ switching was observed to take place at $0.26 \mathrm{~V}$ and $\mathrm{P}$ to $\mathrm{AP}$ switching at $-0.38 \mathrm{~V}$ with a spin-polarized current density $\eta \mathrm{J}=28.5 \mathrm{MA} / \mathrm{cm}^{2}$, given a $10 \mathrm{~ns}$ pulse. Using $H_{K}$ of 500Oe, $M_{S}$ of $1050 \mathrm{emu} / \mathrm{cc}$ and Gilbert damping of 0.02 in our LLG model[55], our unified model reproduces these results. Indeed, with $\eta I=28.5 \mathrm{MA} / \mathrm{cm}^{2}$, we find a current pulse of at least $10 \mathrm{~ns}$ is needed for switching. For $\mathrm{P}$ to AP switching, voltages greater than $-0.38 \mathrm{~V}$ are unable to switch the free ferromagnet within 10ns as seen in Fig 2.7. Similarly for AP to P switching, voltages weaker than $0.26 \mathrm{~V}$ are unable to switch within $10 \mathrm{~ns}$ (Fig. 2.8). Our NEGF currents that agree with the experimental values are thereafter incorporated into the simplified LLG solver above, through the $I$ term that enters the torque.

Similarly, the modified Simmons' model was used to benchmark the same experiment. Further details on this benchmarking will be given in Chapter 5 . 

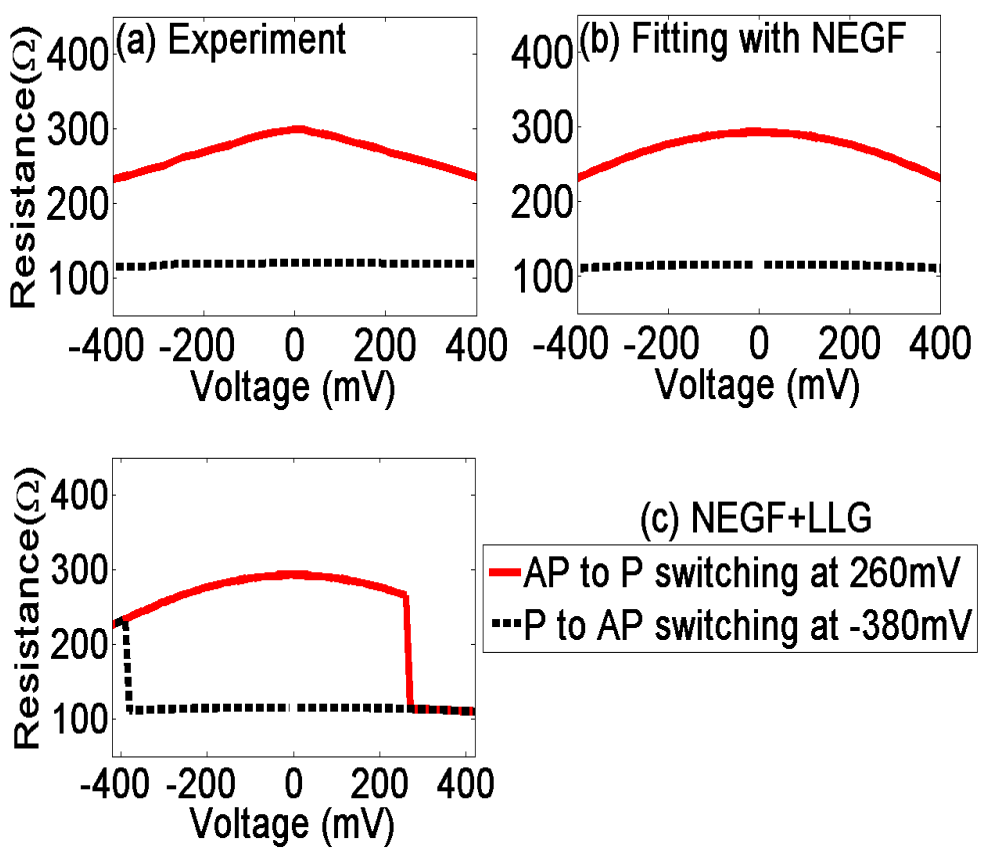

Figure 2.6: (a) Resistance vs voltage data for an in-plane $\mathrm{CoFeB} / \mathrm{MgO} / \mathrm{CoFeB}$ MTJ where the magnetization of the free layer is held constant with an external applied field. (b) The NEGF model was used to fit the experimental data and the transport parameters extracted from the fitting are $E_{F}=2.25 \mathrm{eV}, \mathrm{U}=1 \mathrm{eV}$, width $=1 \mathrm{~nm}, m_{c}=1.3, m_{\text {barr }}=0.32$ and $\delta=1.96 \mathrm{eV}$. (c) The parameters were then used to calculate torque that served as an input into the LLG solver. An $H_{K}$ of $500 \mathrm{Oe}, M_{S}$ of $1050 \mathrm{emu} / \mathrm{cc}$ and Gilbert damping of 0.02 were used. The combined model predicted the $\mathrm{P}$ to AP switching to take place at $-380 \mathrm{mV}$ and $\mathrm{AP}$ to $\mathrm{P}$ switching to take place at $260 \mathrm{mV}$ for a pulse width of $10 \mathrm{~ns}$ and initial angle of $0.04 \mathrm{rad}$. The switching voltage of the comprehensive model matched that of the experiment.
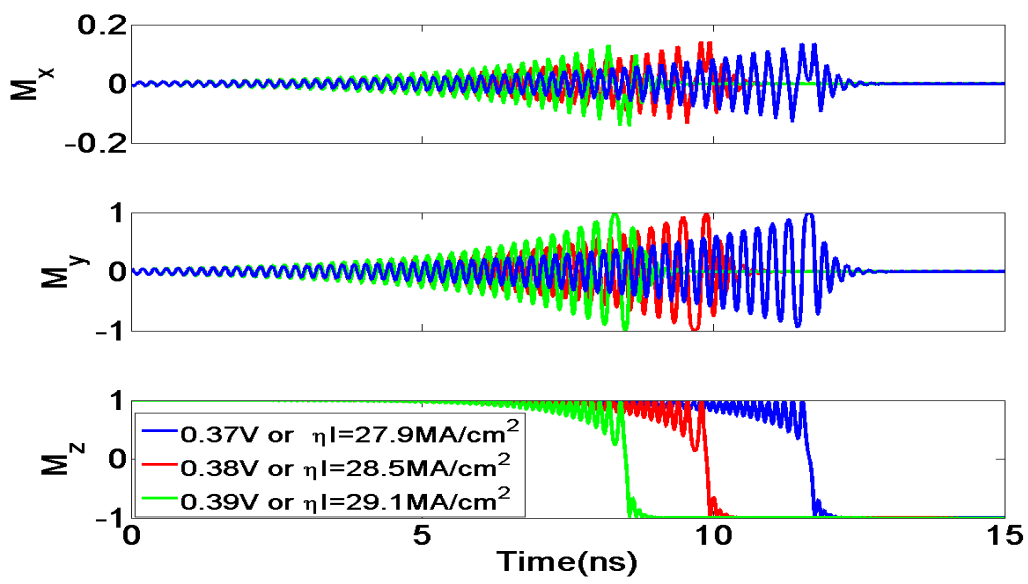

Figure 2.7: Parallel to anti-parallel switching takes place in an in-plane $\mathrm{CoFeB} / \mathrm{MgO} / \mathrm{CoFeB}$ magnetic tunnel junction at $-0.38 \mathrm{~V}$ given a $10 \mathrm{~ns}$ pulse of spin current, with a spin current density, $\eta \mathrm{J}=-28.5 \mathrm{MA} / \mathrm{cm}^{2}$. A current pulse of at least $10 \mathrm{~ns}$ is needed for switching at $-0.38 \mathrm{~V}$. Voltages greater than $-0.38 \mathrm{~V}$ will not be able to switch the free ferromagnet within $10 \mathrm{~ns}$ as shown in the figure. $H_{K}$ of $39.78 \mathrm{KA} / \mathrm{m}, M_{S}$ of $1050 \mathrm{KA} / \mathrm{m}$ and Gilbert damping of 0.02 were used. The magnetization of the free ferromagnet switches from $\theta \approx 0$ to $\theta \approx \pi$. $M_{Z}=\cos \theta$. The simulation results agree quantitatively with experiments. 

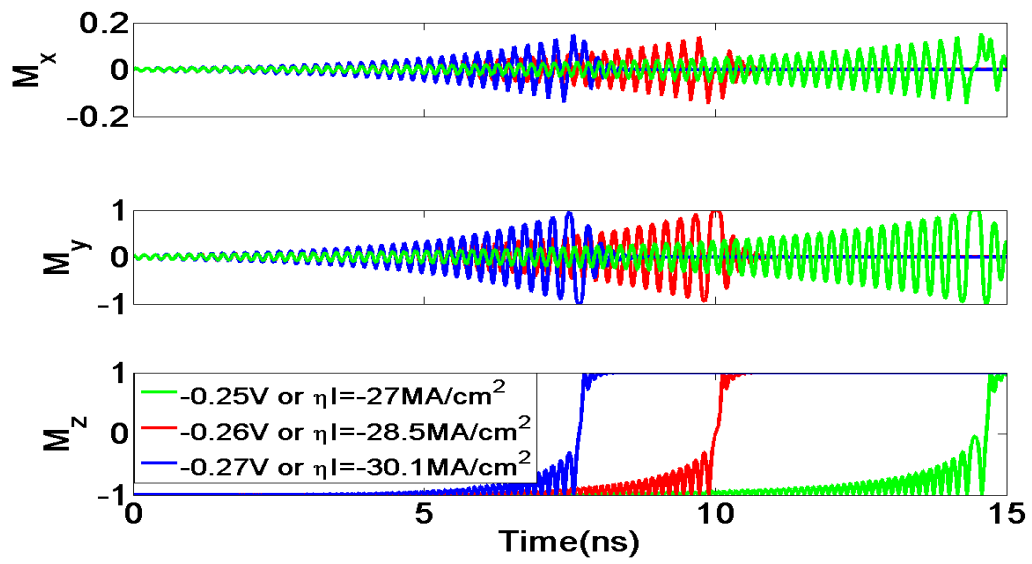

Figure 2.8: Anti-parallel to parallel switching takes place in an in-plane $\mathrm{CoFeB} / \mathrm{MgO} / \mathrm{CoFeB}$ magnetic tunnel junction at $0.26 \mathrm{~V}$ given a $10 \mathrm{~ns}$ pulse of spin current, with spin current density $\eta \mathrm{J}=28.5 \mathrm{MA} / \mathrm{cm}^{2}$. A current pulse of at least $10 \mathrm{~ns}$ is needed for switching at $0.26 \mathrm{~V}$. Voltages less than $0.26 \mathrm{~V}$ will not be able to switch the free ferromagnet within $10 \mathrm{~ns}$ as shown in the figure. The magnetization of the free ferromagnet switches from $\theta \approx \pi$ to $\theta \approx 0$. The simulation results agree quantitatively with experiments. 


\section{Chapter 3}

\section{Investigating Spin-transfer torque switching using the unified model}

The critical current required for current STT-RAM devices, for spin torque induced switching is still too high for commercial applications, prompting intense investigation into the underlying physical, material and device related issues. Most experiments on spin transfer torque switching show that the parallel (P) to anti-parallel (AP) switching takes place at a higher voltage than AP to $\mathrm{P}$ switching $[56,57,54,58]$. The asymmetry in the switching voltages arises from the fact that the torque exerted on the free layer is dependent on the voltage dependent polarization of the fixed layer, as seen in numerical simulations before [51, 59].

In this chapter, we investigate both $\mathrm{P}$ to $\mathrm{AP}$ and $\mathrm{AP}$ to $\mathrm{P}$ switching using both (a) free electron approximation and (b) a predictive Non Equilibrium Greens Function (NEGF) + Landau-Lifshitz-Gilbert (LLG) STT-RAM model and show that with higher polarized ferromagnetic contacts, the switching voltage decreases and becomes symmetric. In section 3.1, through the help of the predictive NEGF+LLG model, we revisit the origin of spin-torque switching in the free layer by studying spin accumulation near the insulator and free-layer interface prior to switching. In section 3.2, by free electron approximation, we prove that the torque exerted at the free layer is indeed dependent on the voltage dependent polarization of the fixed layer. Increasing the polarization of the contact results in increased torque and more symmetrical P to AP and AP to $\mathrm{P}$ switching in respect to the voltage needed to make the switch for a given pulse width. STT-RAMs with high polarized ferromagnetic contacts will not only have high tunneling magnetoresistance, but energy-efficient $\mathrm{AP}$ to $\mathrm{P}$ and $\mathrm{P}$ to AP switching. Furthermore, the NEGF + LLG solver is used to validate the prediction. Using the NEGF+LLG model, we see while the in-plane spin-transfer torque $\left[\hat{m} \times\left(\hat{m} \times \hat{m}_{p}\right)\right]$ is most crucial to the switching, the perpendicular torque $\left[\hat{m} \times \hat{m}_{p}\right]$ plays a part as well. 


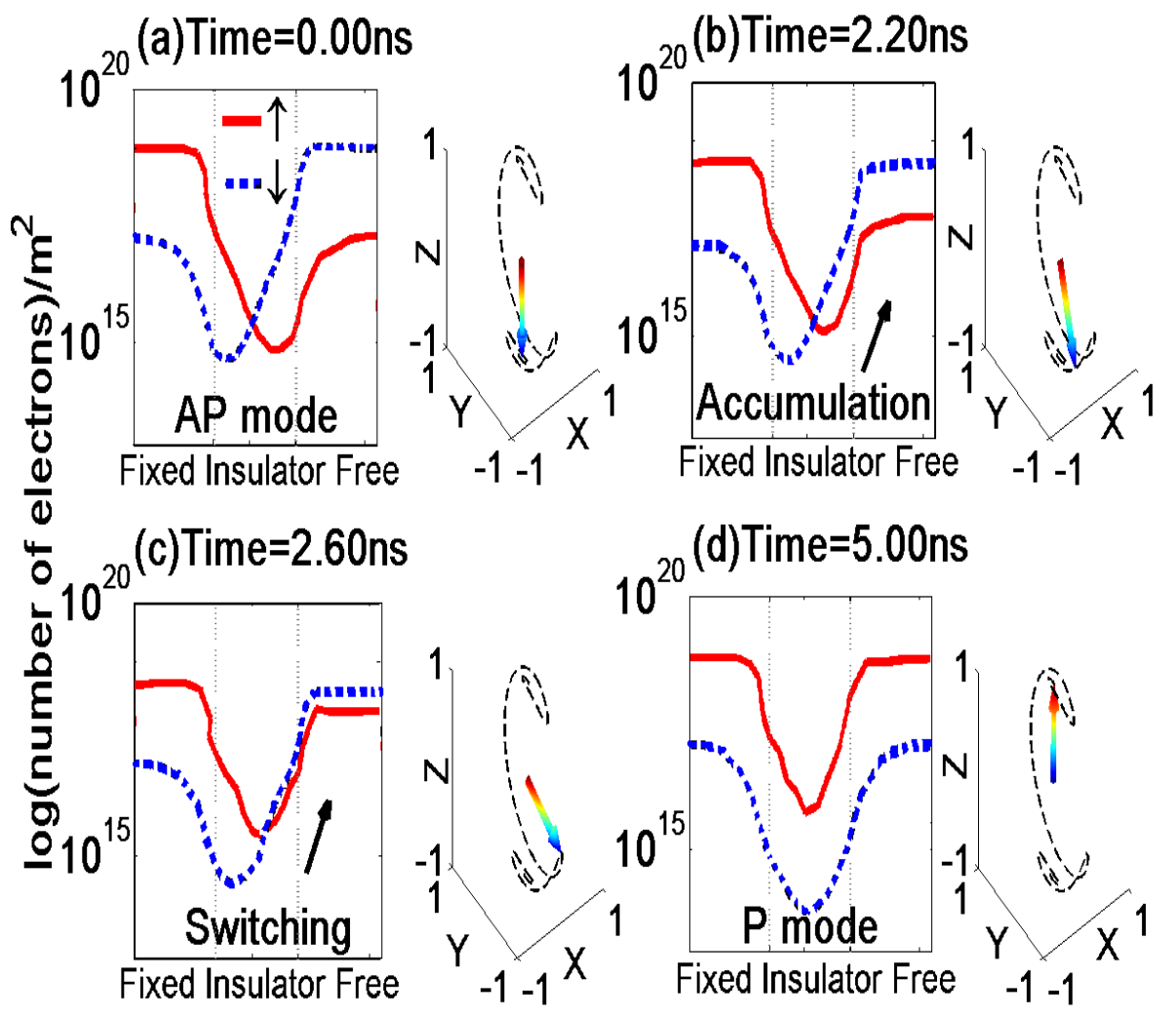

Figure 3.1: AP to $\mathrm{P}$ switching at $0.3 \mathrm{~V}$. The free layer and insulator interface is at position $2 \mathrm{~nm}$. (a)Time=0ns: The free-layer is anti-parallel to the fixed layer, (b) Time=2.2ns: Majority electrons with respect to the fixed layer tunnel through the barrier and accumulate at the insulator-free layer interface. The accumulated majority electrons exerts a torque on the free layer which causes its magnetization to switch. (c)Time=2.6ns: The free layer switching from anti-parallel to parallel configuration. (d)Time=5ns: The final magnetization of the free layer is parallel to the fixed layer.

\subsection{How parallel to anti-parallel and anti-parallel to parallel}

\section{switchings occur}

With the help of the predictive NEGF+LLG STT-RAM model, we show how anti-parallel to parallel and parallel to anti-parallel switching occur in the free layer. For an applied positive voltage, majority electrons with respect to the fixed layer tunnel through the barrier and accumulate at the insulator-free layer interface as shown in Fig 3.1b. The accumulated majority electrons exerts a torque on the free layer which causes its magnetization to switch. After enough majority spin is accumulated to exert torque, the free layer starts switching from anti-parallel to parallel configuration at 2.6ns. By 5 ns, the magnetization of the free layer has completed its switching from anti-parallel to parallel mode.

Conversely for parallel to anti-parallel switching, a negative voltage is applied (Fig. 3.2). At 0 ns, 


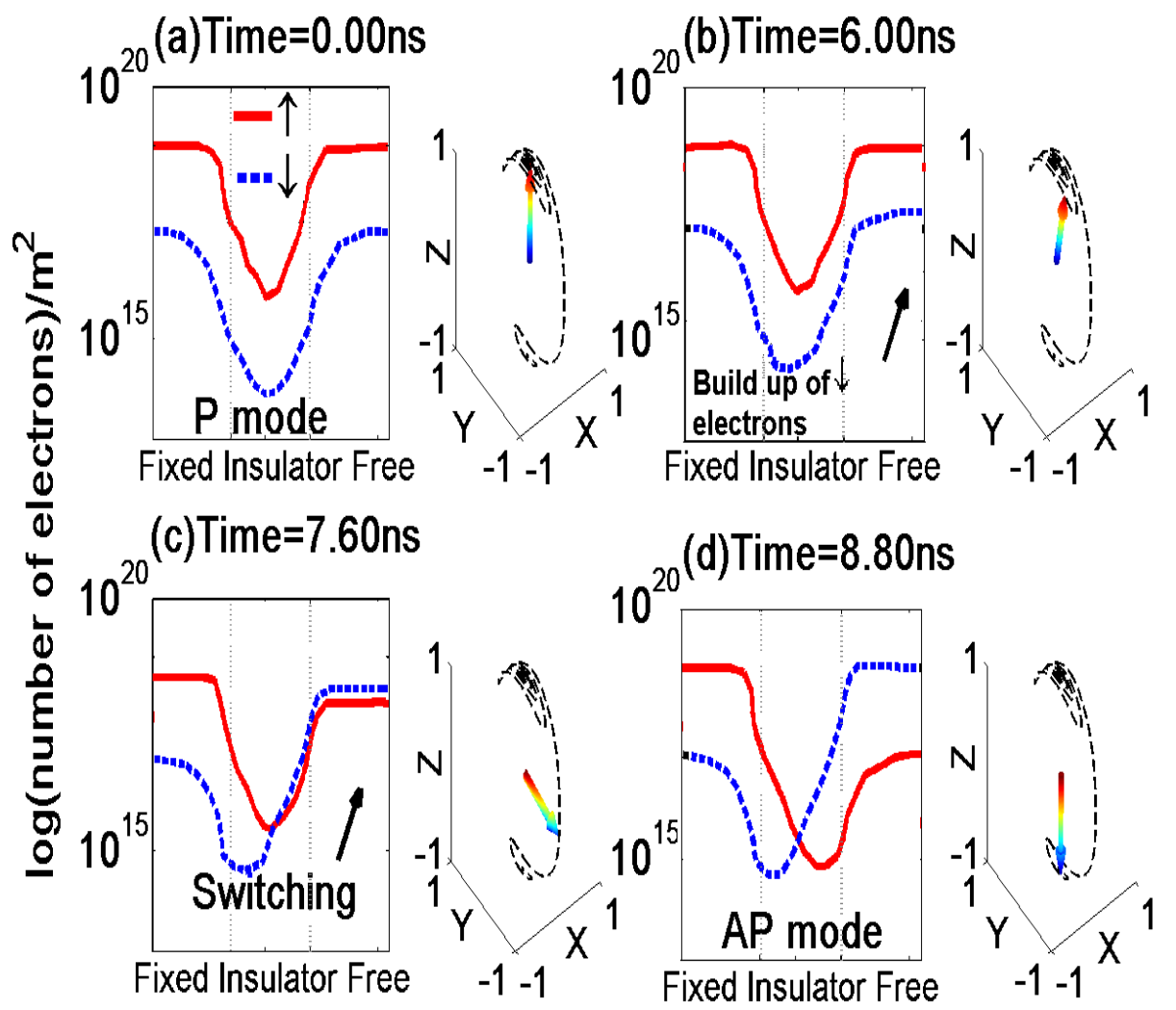

Figure 3.2: $\mathrm{P}$ to AP switching at $-0.4 \mathrm{~V}$. The free layer and insulator interface is at position $2 \mathrm{~nm}$. (a)Time=0ns: The free-layer is parallel to the fixed layer, (b) Time $=6 \mathrm{~ns}$ : Minority electrons with respect to the free layer tunnel through the barrier, reflect back from the fixed layer and insulater interface, and accumulate at the insulator-free layer interface. The accumulated minority electrons exerts a torque on the free layer which causes its magnetization to switch. (c)Time=7.6ns: The free layer switching from parallel to anti-parallel configuration. (d)Time=8ns: The final magnetization of the free layer is anti-parallel to the fixed layer.

the free-layer is parallel to the fixed layer. At 6ns, there is an accumulation of minority electrons at the insulator-free layer interface. The accumulated minority electrons exerts a torque on the free layer which causes its magnetization to switch. After enough majority spin is accumulated to exert torque, the free layer starts switching from anti-parallel to parallel configuration at 7.6 ns. By 8 ns, the magnetization of the free layer has completed its switching from parallel to anti-parallel mode.

Two points to be noted here. First, given the same absolute value of voltage, the anti-parallel to parallel switching occurs much faster than parallel to anti-parallel switching. We will see in the next section using free-electron approximation, that the difference in the switching speed is due to the difference in the operating polarization at the two switching voltages. Second point to be noted in the origin of the accumulated minority carriers during the $\mathrm{P}$ to AP switching which decreases the magnetization of the free layer. 

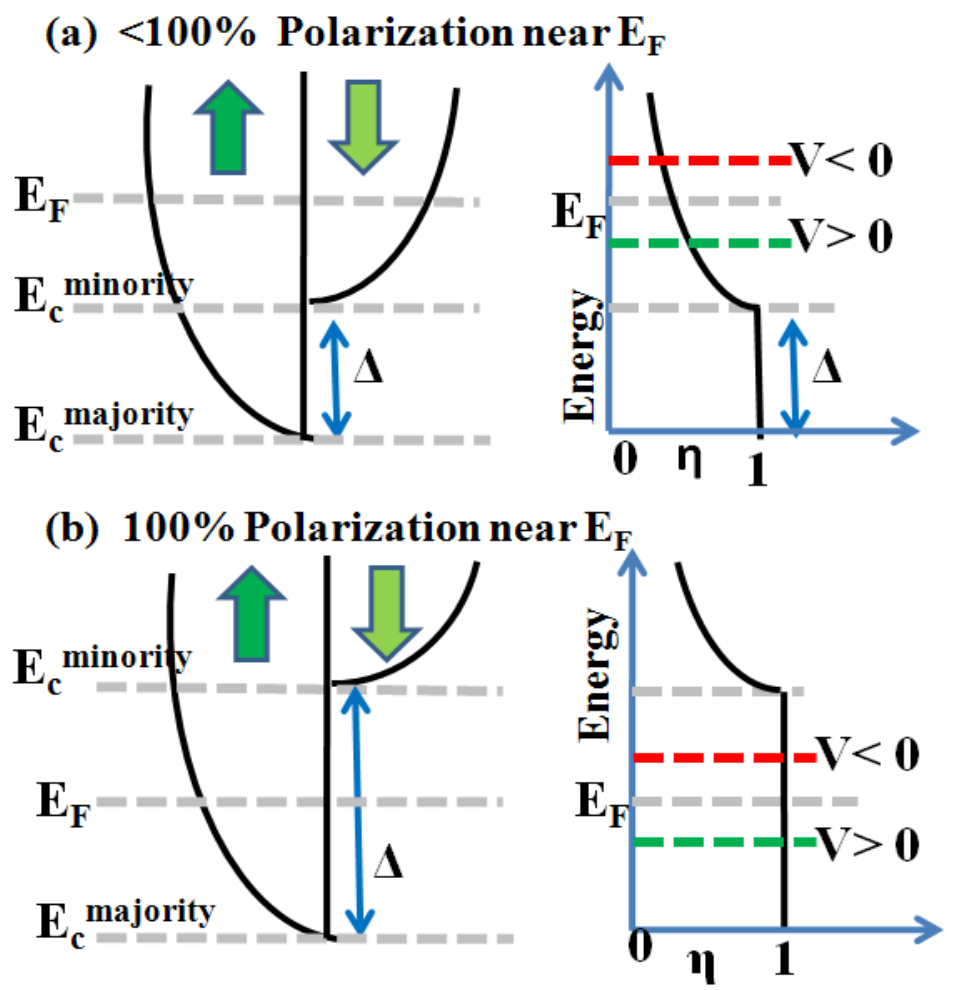

Figure 3.3: Voltage dependent polarization. Effective polarization at the given energy, $\eta=\left(n_{\uparrow}-n_{\downarrow}\right) /\left(n_{\uparrow}+n_{\downarrow}\right)$, where $n_{\uparrow}$ and $n_{\downarrow}$ are the number of majority and minority electrons respectively. (a) When $\Delta<E_{F}, \eta$, at $E_{F}$ is less than $100 \% . \eta(V>0)$ is greater than $\eta(V<0)$. Therefore, the effective polarization during AP to $\mathrm{P}$ switching is higher than that during $\mathrm{P}$ to $\mathrm{AP}$ switching. (b) When $\Delta>E_{F}, \eta$, at $E_{F}$ is $100 \%$. $\eta(V>0)$ is equal to $\eta(V<0)$. Therefore, the effective polarization during AP to $\mathrm{P}$ switching is the same of that during $\mathrm{P}$ to AP switching.

\subsection{AP to $\mathrm{P}$ and $\mathrm{P}$ to AP switching voltages asymmetry}

In section 2.2.2, it has been proved that while the torque in the right contact adds angular momentum to induce anti-parallel to parallel switching for positive bias across the tunnel junction, there is a reciprocal torque in the left contact that removes angular momentum and induces parallel to anti-parallel switching. The torque at the left layer is dependent on the polarization of the left layer, $k_{\uparrow}^{+}-k_{\downarrow}^{+}$. Therefore, in a STT-RAM cell, the intensity of the torque at a given voltage is dependent at the polarization of that given voltage as shown in Fig 3.3a. For less than $100 \%$ polarized contacts, $\Delta<E_{F}$. Polarization, $\eta(V>0)$ is greater than $\eta(V<0)$. Therefore, the effective polarization during AP to $\mathrm{P}$ switching is higher than that during $\mathrm{P}$ to $\mathrm{AP}$ switching.

At $100 \%$ polarization, where $k_{\downarrow}=k_{\downarrow}^{+}=0$, the torques at the two end are equal which should result in symmetric $\mathrm{P}$ to AP and AP to $\mathrm{P}$ switching. The torques at the right contact is dependent on $k_{\uparrow}$ while the torque at the left contact is dependent on $k_{\uparrow}^{+}$. Both the torques are more intensified than the torque generated 

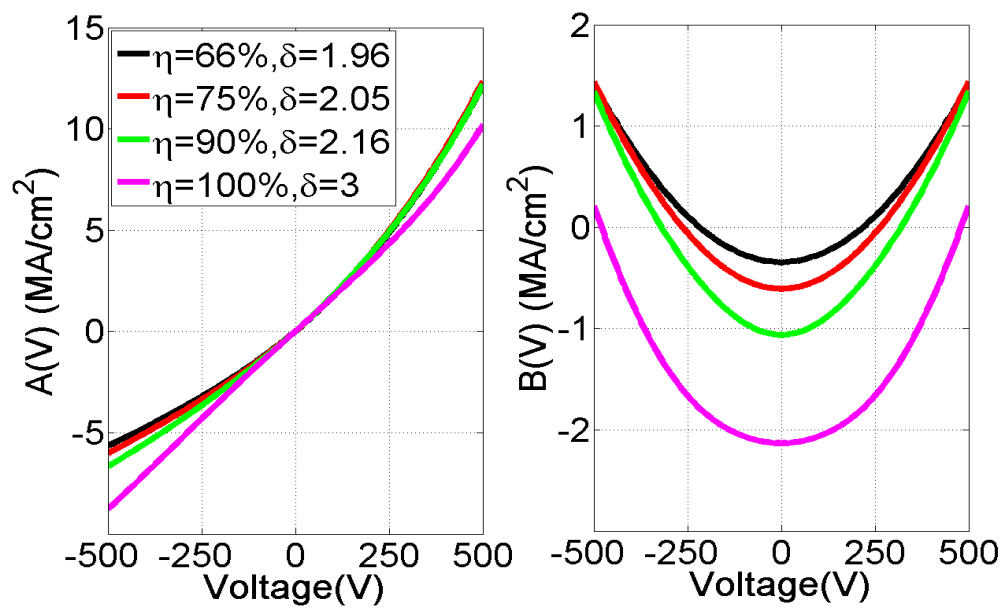

Figure 3.4: As the fixed layer polarization $(\eta$ and $\delta$ ) increases, the spin-transfer torque, $\mathrm{A}(\mathrm{V})$ becomes more symmetric with voltage. The perpendicular torque has a quadratic dependence on the voltage, $B(V)=B_{0}+B_{1} V^{2}$. The term $B_{0}$ is the exchange coupling between the fixed and the free layers at zero voltage and intensifies as $\eta$ increases. Positive value of $\mathrm{B}(\mathrm{V})$ prefers parallel configuration in the MTJ while negative value prefers the anti-parallel configuration. When $\eta=66 \%, \mathrm{~B}$ is negative near zero voltage but change to positive as voltage increases. The sign change is explained in Fig. 7 . When $\eta=99 \%, \mathrm{~B}(\mathrm{~V})$ is mostly negetive.

in contacts with less than $100 \%$ polarization.

Region III:

$$
\begin{aligned}
& J_{I I I, X}=\frac{8 k_{\uparrow}^{2} q \hbar e^{-2 \kappa w} k_{\uparrow}^{+} \sin (\theta)}{m\left(\kappa^{2}+k_{\uparrow}^{2}\right)\left(\kappa^{2}+k_{\uparrow}^{+2}\right)}\left[\kappa^{2} \cos \left(k_{\uparrow}^{+} x\right)+\kappa k_{\uparrow}^{+} \sin \left(k_{\uparrow}^{+} x\right)\right] \\
& J_{I I I, Y}=\frac{8 i k_{\uparrow}^{2} q \hbar e^{-2 \kappa w} k_{\uparrow}^{+} \sin (\theta)}{m\left(\kappa^{2}+k_{\uparrow}^{2}\right)\left(\kappa^{2}+k_{\uparrow}^{+2}\right)}\left[\kappa^{2} \cos \left(k_{\uparrow}^{+} x\right)+\kappa k_{\uparrow}^{+} \sin \left(k_{\uparrow}^{+} x\right)\right]
\end{aligned}
$$

Region I:

$$
\begin{aligned}
& J_{I, X}=\frac{-8 k_{\uparrow}^{2} q \hbar e^{-2 \kappa w} k_{\uparrow}^{+} \sin (\theta)}{m\left(\kappa^{2}+k_{\uparrow}^{2}\right)\left(\kappa^{2}+k_{\uparrow}^{+2}\right)}\left[\kappa^{2} \cos \left(k_{\uparrow} x\right)+\kappa k_{\uparrow} \sin \left(k_{\uparrow} x\right)\right] \\
& J_{I, Y}=\frac{-8 i k_{\uparrow}^{2} q \hbar e^{-2 \kappa w} k_{\uparrow}^{+} \sin (\theta)}{m\left(\kappa^{2}+k_{\uparrow}^{2}\right)\left(\kappa^{2}+k_{\uparrow}^{+2}\right)}\left[\kappa^{2} \cos \left(k_{\uparrow} x\right)+\kappa k_{\uparrow} \sin \left(k_{\uparrow} x\right)\right]
\end{aligned}
$$

A more realistic calculation is done using NEGF and a macrospin solver that solves the Landau-LifshitzGilbert equation to emulate the switching of the free right layer. The traverse momentum in the $\mathrm{Y}$ and $\mathrm{Z}$ directions and potential drop in the barrier are properly modeled. Fig. 3.4 shows the spin-transfer and perpendicular torques currents for different polarization using the NEGF model: (i) 66\% polarization or $\delta=1.95 \mathrm{eV}$, (ii) $75 \%$ polarization or $\delta=2.05 \mathrm{eV}$,(ii) $90 \%$ polarization or $\delta=2.15 \mathrm{eV}$, and(iV) $100 \%$ polarization or $\delta=3 \mathrm{eV}$. As polarization increases, the spin-transfer torque, dependent of the effective polarization of the fixed contact, becomes more symmetric, as indicated by the results from free-electron approximation in the previously. At $100 \%$ polarization, the torque is symmetric with respect to voltage. 

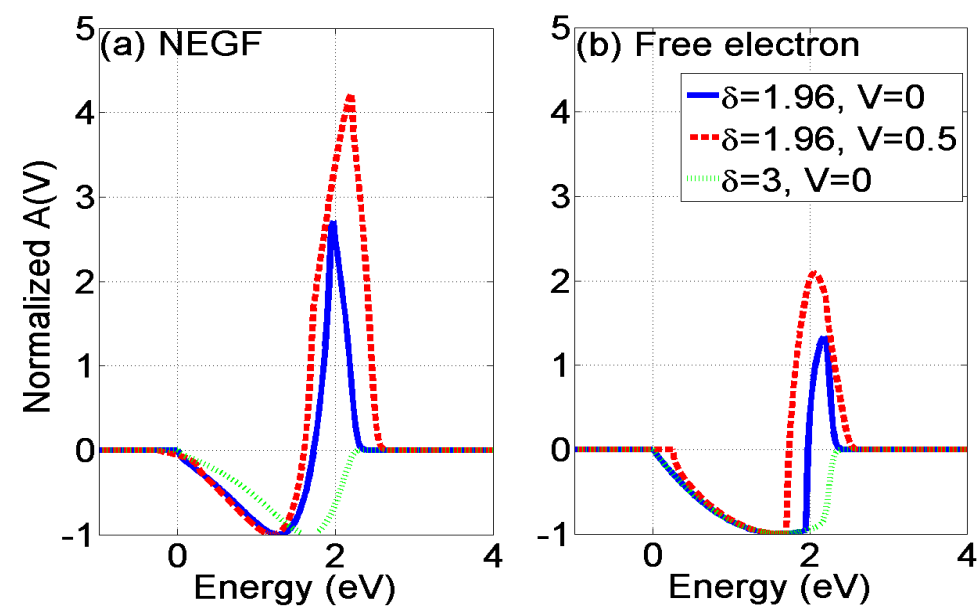

Figure 3.5: Normalized perpendicular torque with respect to energy for (a) NEGF and (b) free electron models. According to the free electron model, the perpendicular torque, $J_{Y}$ is dependent on $\left(\kappa^{2}-k_{\uparrow} k_{\downarrow}\right) \approx$ $-(2 m / \hbar)(U-E-(E-q V) \sqrt{1-\delta /(E-q V)}$. Torque for energy below $\delta-q V$ is negative and for energy above $\delta-q V$ is positive. For $\delta=1.96 \mathrm{eV}$ and applied voltage of zero, the torque switches sign at energy $1.96 \mathrm{eV}$. Sum of the perpendicular torque over the entire energy range is negative. For $\delta=1.96 \mathrm{eV}$ and applied voltage of $0.5 \mathrm{~V}$, the torque switches sign at energy $1.71 \mathrm{eV}$. Sum of the perpendicular torque over the entire energy range is positive. For $\delta=3 \mathrm{eV}$ and applied voltage of zero, the torque does not switch sign. Sum of the perpendicular torque over the entire energy range is negative.

\subsection{Field-like term}

The perpendicular torque has a quadratic dependence on the voltage, $B(V)=B_{0}+B_{1} V^{2}$ (Fig. $\left.3.4 \mathrm{~b}\right)$. The term $B_{0}$ is the exchange coupling between the fixed and the free layers at zero voltage and intensifies as $\eta$ increases. When $\eta=66 \%$, B is negative near zero voltage but change to positive as voltage increases. When $\eta=99 \%, \mathrm{~B}(\mathrm{~V})$ is mostly negative. Fig 7 shows the normalized perpendicular torque with respect to energy for (a) NEGF and (b) free electron models. According to the free electron model, the perpendicular torque, $J_{Y}$ is dependent on $\left(\kappa^{2}-k_{\uparrow} k_{\downarrow}\right) \approx-(2 m / \hbar)(U-E-(E-q V) \sqrt{1-\delta /(E-q V)}$. Torque for energy below $\delta-q V$ is negative and for energy above $\delta-q V$ is positive. For $\delta=1.96 \mathrm{eV}$ and applied voltage of zero, the torque switches sign at energy $1.96 \mathrm{eV}$ and sum of the perpendicular torque over the entire energy range is negative. For $\delta=1.96 \mathrm{eV}$ and applied voltage of $0.5 \mathrm{~V}$, the torque switches sign at energy $1.71 \mathrm{eV}$ and sum of the perpendicular torque over the entire energy range is positive. For $\delta=3 \mathrm{eV}$ and applied voltage of zero, the torque does not switch sign and sum of the perpendicular torque over the entire energy range is negative.

Positive value of $\mathrm{B}(\mathrm{V})$ prefers parallel configuration in the MTJ while negative value prefers the anti-parallel configuration in in-plane free layers as seen in Fig. 3.6. The critical switching current becomes,

$$
I_{c}-\alpha B_{1}=\frac{1}{\eta} \frac{2 q}{\hbar} \alpha \Omega H_{K} M_{S}\left(1+\frac{2 \pi M_{S}}{H_{K}}+\frac{H}{H_{K}}\right)
$$




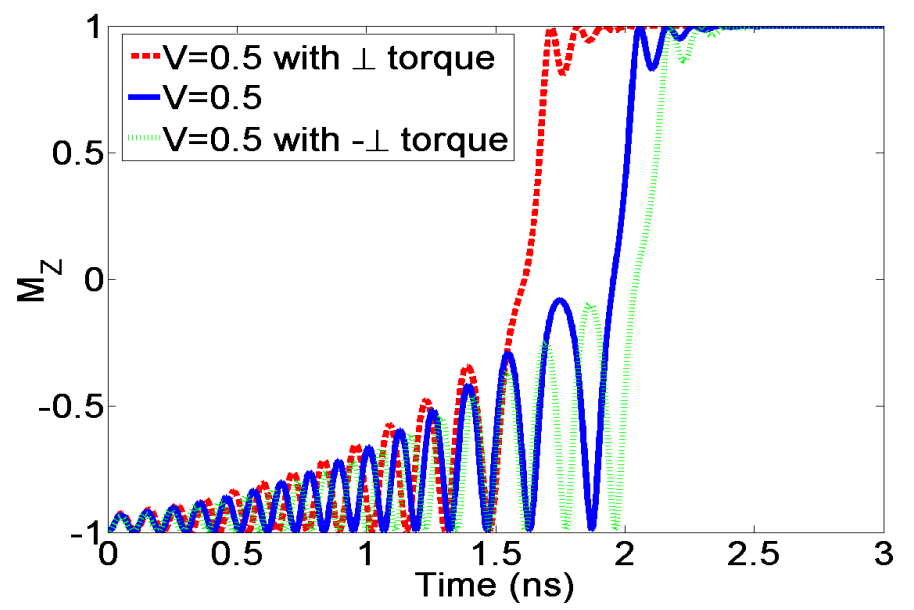

Figure 3.6: AP to $\mathrm{P}$ switching in an in-plane magnetic layer. Magnetic properties of CoFeB, $H_{K}$ of $500 \mathrm{Oe}$, $M_{S}$ of $1050 \mathrm{emu} / \mathrm{cc}$ and $\alpha$ of 0.02 are used. Positive field-like torque helps AP to P switching while a negative field-like torque delays the switching.

where $\mathrm{H}$ is the field due to perpendicular torque at zero voltage, $H=\left(H_{H} \hbar \eta B_{0}\right) /(4 q \Omega K)$, and $B_{1}$ is the additional perpendicular torque due to voltage applied. The perpendicular torque at zero voltage increases the thermal stability of the AP configuration and decreases the stability at the P configuration[60].

$$
\begin{aligned}
& \Delta_{A P}=\frac{H_{K} M_{S} \Omega}{2 K_{B} T}\left(1+\frac{H}{H_{K}}\right)^{2} \\
& \Delta_{P}=\frac{H_{K} M_{S} \Omega}{2 K_{B} T}\left(1-\frac{H}{H_{K}}\right)^{2}
\end{aligned}
$$

For an in-plane free layer of $H_{K}=500 \mathrm{Oe}, M_{S}=1050 \mathrm{emu} / \mathrm{cc}$ and thickness of $2 \mathrm{~nm}$, the H fields are $22.68 \mathrm{Oe}$, 39.730e, 69.51e, and 139.280e for $66 \%, 75 \%, 90 \%$ and $100 \% \eta$ respectively. In perpendicular free layer, the torque only effects the precession of the magnetization and does not affect the switching speed (Fig. 3.7).

With the parameters extracted from the experimental benchmark in section 2.3, MTJ with increasing polarized contacts,(increasing $\delta$ ) are studied in in-plane (Fig. 3.8) and perpendicular (Fig. 3.9) Fig 10 shows $\mathrm{AP}$ to $\mathrm{P}$ and $\mathrm{P}$ to AP switching voltages for in-plane contacts. Magnetic properties of $\mathrm{CoFeB}, H_{K}$ of $500 \mathrm{Oe}$, $M_{S}$ of $1050 \mathrm{emu} / \mathrm{cc}$ and $\alpha$ of 0.02 are used. Blue line indicates switching voltages taking both spin-transfer and field-like torques into account, while the red lines account only for spin-transfer torques. For lower values of $\Delta(<2.15 \mathrm{eV})$, field-like torque, being positive, hampers $\mathrm{P}$ to AP switching and helps $\mathrm{AP}$ to $\mathrm{P}$ switching. For higher values of $\Delta(>2.15 \mathrm{eV})$, field-like torque, being negative, helps $\mathrm{P}$ to AP switching and hampers $\mathrm{AP}$ to $\mathrm{P}$ switching. With field-like torque taken into account, voltage asymmetry, $V_{A P t o P} / V_{P t o A P}$ increases with increasing $\Delta$. Without the field-like torque, voltage asymmetry increases and stabilizes at 1 for higher polarization, indication symmetric in-plane torque switching. Fig 11 shows AP to $\mathrm{P}$ and $\mathrm{P}$ to AP switching 


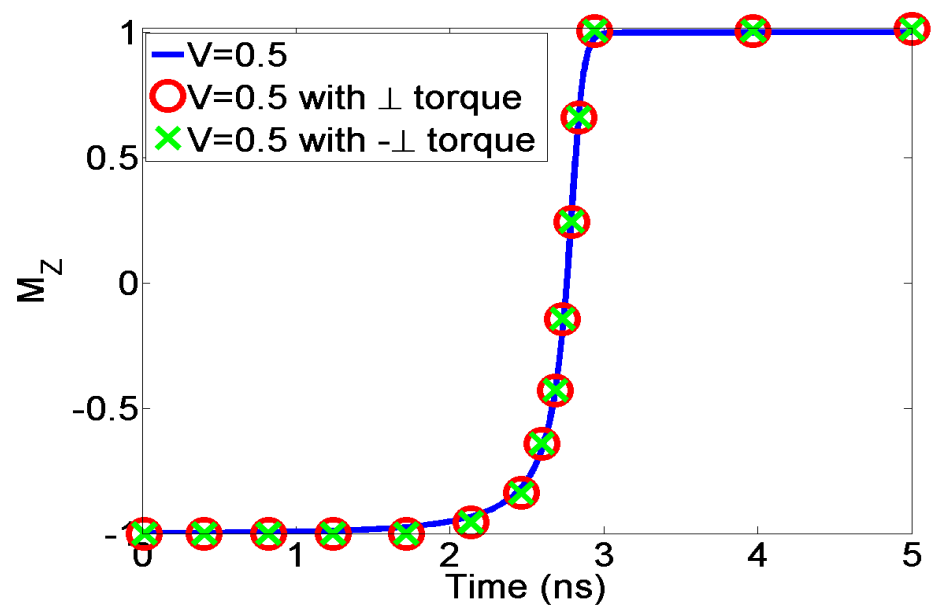

Figure 3.7: AP to $\mathrm{P}$ switching in a perpendicular magnetic layer. Magnetic properties of CoPd, $H_{K}$ of 20,0000e, $M_{S}$ of $450 \mathrm{emu} / \mathrm{cc}$ and $\alpha$ of 0.1 are used. The field-like torque do not effect the switching delay.

voltages for perpendicular contacts. Magnetic properties of $\mathrm{CoPd}, H_{K}$ of $20,000 \mathrm{Oe}, M_{S}$ of $450 \mathrm{emu} / \mathrm{cc}$ and $\alpha$ of 0.1 are used $[61,62]$. The field-like torque do not effect the switching voltages and therefore, the switching voltage is dependent just on the polarization dependent in-plane torque. With or without field-like torque, switching voltage becomes more symmetric with increasing $\delta$.

Through the help of the predictive NEGF+LLG model, we revisit the origin of spin-torque switching in the free layer by studying spin accumulation near the insulator and free-layer interface prior to switching.For an applied positive voltage, majority electrons with respect to the fixed layer tunnel through the barrier and accumulate at the insulator-free layer, exerting a torque on the free layer which causes its magnetization to switch from AP to P. Conversely $\mathrm{P}$ to AP switching, a negative voltage is applied and there is an accumulation of minority electrons at the insulator-fixed layer interface. The accumulated minority electrons exerts a torque on the free layer which causes its magnetization to switch. The difference in the switching speed is due to the difference in the operating polarization at the two switching voltages which is studied in detail using both the free electron and the NEGF models. We show that with with higher polarized ferromagnetic contacts, the switching voltage decreases and becomes symmetric in perpendicular free layers. However, due to the field-like torque in in-plane materials, the asymmetry still exists. 


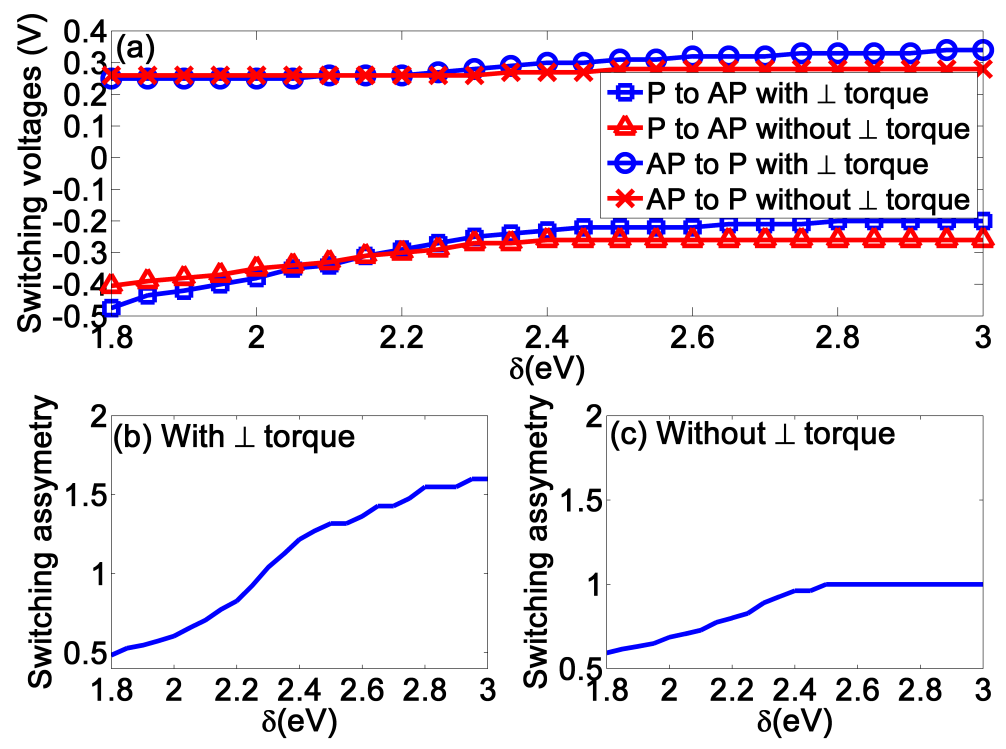

Figure 3.8: (a) AP to $\mathrm{P}$ and $\mathrm{P}$ to AP switching voltages for in-plane contacts. Magnetic properties of CoFeB, $H_{K}$ of $500 \mathrm{Oe}, M_{S}$ of $1050 \mathrm{emu} / \mathrm{cc}$ and $\alpha$ of 0.02 are used. Blue line indicates switching voltages taking both spin-transfer and field-like torques into account, while the red lines account only for spin-transfer torques. For lower values of $\delta(<2.15 \mathrm{eV})$, field-like torque, being positive, hampers $\mathrm{P}$ to AP switching and helps $\mathrm{AP}$ to $\mathrm{P}$ switching. For higher values of $\delta(>2.15 \mathrm{eV})$, field-like torque, being negative, helps $\mathrm{P}$ to $\mathrm{AP}$ switching and hampers AP to $\mathrm{P}$ switching. (b) With field-like torque taken into account, voltage asymmetry, $V_{A P t o P} / V_{\text {PtoAP }}$ increases with increasing $\delta$. (c) Without the field-like torque, voltage asymmetry increases and stabilizes at 1 for higher polarization, indication symmetric spin-transfer torque switching.
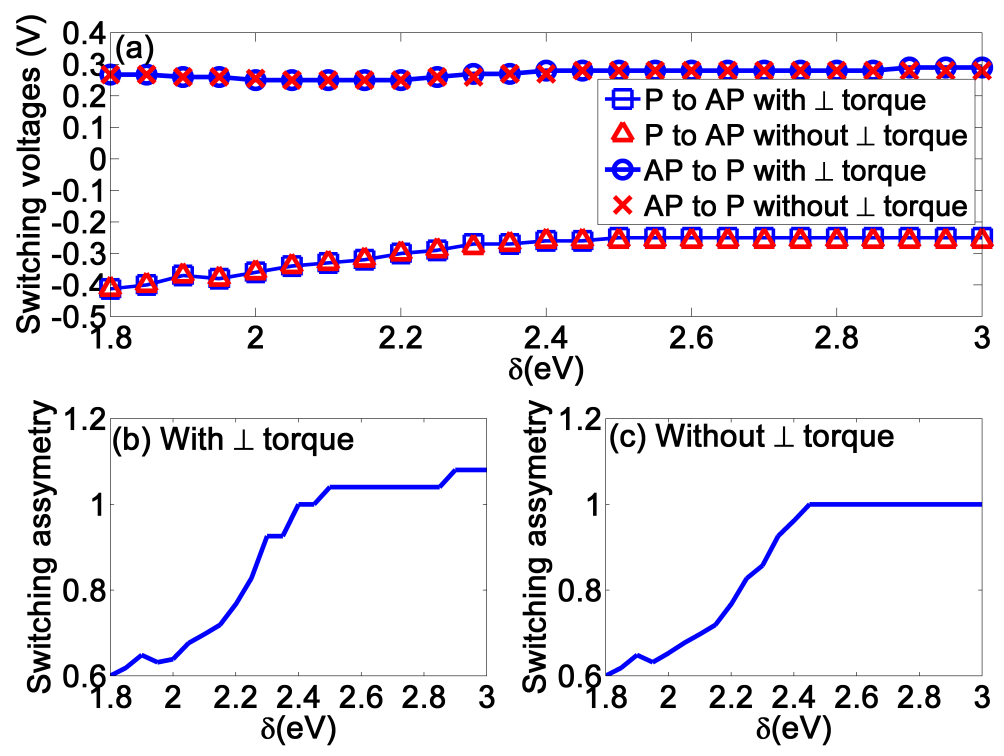

Figure 3.9: (a) AP to $\mathrm{P}$ and $\mathrm{P}$ to AP switching voltages for perpendicular contacts. Magnetic properties of $\mathrm{CoPd}, H_{K}$ of $20,000 \mathrm{Oe}, M_{S}$ of $450 \mathrm{emu} / \mathrm{cc}$ and $\alpha$ of 0.1 are used. The field-like torque do not effect the switching voltages and therefore, the switching voltage is dependent just on the spin-transfer torque. (b) and (c): With or without field-like torque, switching voltage becomes more symmetric with increasing $\delta$. 


\section{Chapter 4}

\section{Comparative material issues for faster switching in STT-RAM}

The critical current densities for spin torque switching in STT-RAMs is nonetheless too high for commercial applications, prompting intense investigation into material properties of the free layer[63]. The energy dissipation during the spin-transfer torque switching is given by $I^{2} R \tau$, where $\mathrm{I}$ is the current used to induce spin-transfer torque switching, $\mathrm{R}$ is resistance of the MTJ and $\tau$ is the total delay time it takes for the magnetization of the free-layer to switch. In this chapter, for a given spin-polarized current density, J, of $2 \mathrm{MA} / \mathrm{cm}^{2}$, we study the switching speed $\tau$ of different classes of magnetic materials, in-plane, perpendicular and partially-perpendicular (Fig 4.5) at 0K temperature. We consider the free layer at the 45nm feature STT-RAM technology, and employ a single domain macro-spin solver that solves the Landau-Lifshitz-Gilbert (LLG) equation [52]. For enough stability against thermally driven switching, in order to retain data for at least 10 years, $\Delta$ needs to be greater than 75 at room temperature [64]. The voltage needed to generate a current of such magnitude, and thus the resistance $R$, will be dependent on the material and insulator properties, such as the effective masses, insulator thickness, band-offset and contact polarizations. Extracting the MTJ resistance corresponding to a given $I$ will require solving the quantum transport problem using the non-equilibrium Green's function formalism (NEGF)[65], or in a simpler incarnation, the Simmons' model for tunneling across a barrier, modified to include its crucial magnetization dependent pre-factors [66, 25]. While such a coupled transport-macromagnetic study is ultimately needed for overall energy-efficiency studies, the aim of this paper is to identify materials for fast switching in STT-RAM within 10ns, with acceptable thermal stability $(\Delta>75)$.

A proper model for spin torque induced switching should include thermal effects, which provide the 

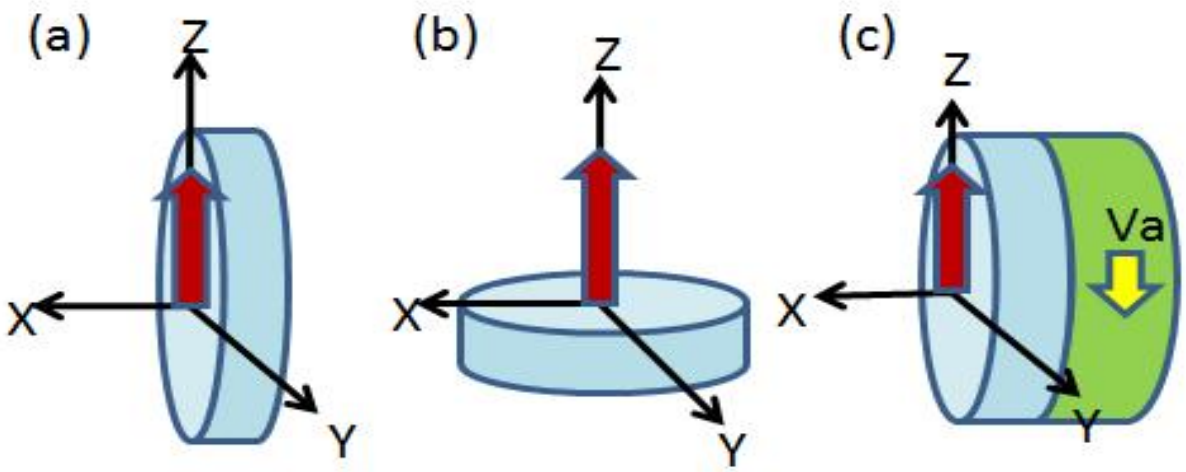

Figure 4.1: Definition of the magnetization of the free ferromagnetic layer in a spherical coordinate system. Magnetization for (a) In-plane material: the easy axis is along the major axis, Z, of the ellipsoidal plane,. (b) Perpendicular material: the easy axis is perpendicular to the disk. (c) Partially-perpendicular: an in-plane disk is capped with a Vanadium layer that reduces the demagnetization anisotropy of the system by anti-ferromagnetic exchange coupling. In all three cases, magnetization prefers to lie near $\theta=0$ (parallel case) or $\theta=\pi$ (anti-parallel case).

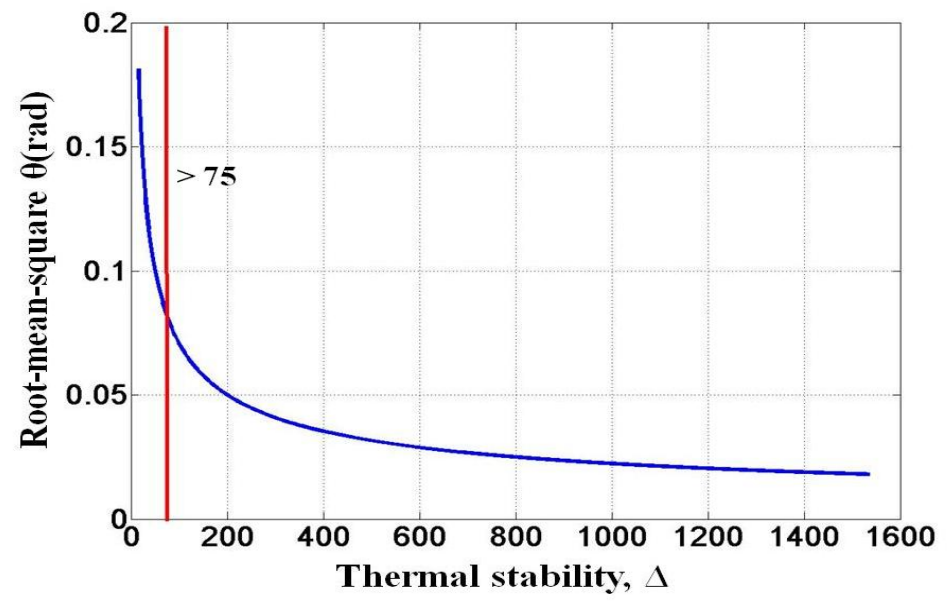

Figure 4.2: Thermal stability, $\Delta$, vs. root-mean-square, $\theta_{r m s}$ (rad) of the free layer nanomagnet. $\Delta$ needs to be greater than 75 for commercial applications. Root-mean-square $\theta_{r m s}(\mathrm{rad})$ identifies the initial angle that we use in our simulations.

initial torque to nudge the magnetization away from stagnation points along the energy landscape (ie, away from strict parallel or anti-parallel configurations), while at the same time hindering the motion of the magnetization on its journey past stagnation. In [25] we include an average over the thermal distribution of the initial angle evaluated using a Fokker-Planck equation. In this work, we simplify this treatment by directly replacing the initial angle with the root mean squared value $\theta_{r m s}$ at $300 \mathrm{~K}$, set by the equipartition theorem (Fig. 4.2). While thermal agitation during the course of the reversal adds some uncertainty to the final delay time, the difference is likely to be small[67]. Therefore, we will disregard the thermal effect during the course of reversal in this chapter. 
Section 4.1 outlines the critical switching currents and thermal stabilities for in-plane, perpendicular and partially-perpendicular ferromagnetic free-layers. Section 4.2 describes the variation in switching speeds across the material classes for a given switching current density and section 4.3 provides a path for the STT-RAM industry for fast, reliable switching.

\subsection{Critical switching currents and thermal stabilities}

\subsubsection{In-plane}

Fig 4.1 defines our choice of axes and angular conventions that we will adopt for the different material classes. For in-plane materials, the easy axis is aligned along the major axis of the ellipsoidal plane (Fig 4.1a). For a $45 \mathrm{~nm}$ feature technology, the free layer ellipsoidal disk has a minor axis of 45nm, major axis of 90nm, and thickness of $2 \mathrm{~nm}$ [64]. The potential energy density for an in-plane system is defined by its magnetocrystalline anisotropy and demagnetization field, $U=K \sin ^{2} \theta+2 \pi M_{S}^{2} \sin ^{2} \theta \cos ^{2} \phi$. The critical current for switching is,

$$
I_{c}=\frac{2 q}{\eta \hbar} \alpha \Omega H_{K} M_{S}\left[1+\frac{2 \pi M_{S}}{H_{K}}\right]
$$

$H_{K}$ is the magnetocrystalline anisotropy field, $M_{S}$ is the saturation magnetization and $\Omega$ is the total volume of the free-layer. The thermal stability is,

$$
\Delta=\frac{H_{K} M_{S} \Omega}{2 K_{B} T}
$$

The switching time required at zero temperature[67] for initial angle, $\theta_{r m s}$, and applied current, I, is

$$
\tau^{-1}=\frac{\alpha \gamma}{\ln \left(\pi / 2 \theta_{r m s}\right)}\left(H_{K}+2 \pi M_{S}\right)\left[\frac{I}{I_{C}}-1\right]
$$

\subsubsection{Perpendicular}

The easy axis is perpendicular to the plane (Fig 4.2b). For a 45nm feature technology, the free layer circular disk has a diameter of $90 \mathrm{~nm}$, and thickness of 2nm [64]. The potential energy density for the system is defined by its magnetocrystalline anisotropy and demagnetization field, $U=K \sin ^{2} \theta+2 \pi M_{S}^{2} \cos ^{2} \theta$. The critical current for switching [68] is

$$
I_{c}=\frac{2 q}{\eta \hbar} \alpha \Omega H_{K} M_{S}\left[1-\frac{4 \pi M_{S}}{H_{K}}\right]
$$


The decreased barrier reduces critical current but also has an adverse effect on thermal stability,

$$
\Delta=\frac{\left(H_{K} M_{S}-4 \pi M_{S}^{2}\right) \Omega}{2 K_{B} T}
$$

Materials with higher saturation magnetization will have less thermal stability, making the range of reliable materials narrower. Most perpendicular materials require epitaxial growth at elevated in-situ temperatures, making them harder to integrate with CMOS processes than in-plane MTJ materials. They also typically have higher damping constants $\alpha$ than in-plane MTJ free layer materials [57, 69, 61, 62].The switching time required at zero temperature for initial angle, $\theta_{r m s}$, and applied current, I, is

$$
\tau^{-1}=\frac{\alpha \gamma}{\ln \left(\pi / 2 \theta_{r m s}\right)}\left(H_{K}-4 \pi M_{S}\right)\left[\frac{I}{I_{C}}-1\right]
$$

\subsubsection{Partially-perpendicular}

An in-plane material is capped anti-ferromagnetically with a Vanadium cap (Fig 4.2c), that reduces the demagnetization field of the disk[70, 58]. The term partially-perpendicular is used because the overall switching barrier is being reduced as in perpendicular materials (by demagnetization field in the later case), but the magnetization still lies in the plane of the elliptical disk. The potential energy density for the system is defined by its magnetocrystalline anisotropy, demagnetization field, and exchange coupling with the capping layer, $U=K \sin ^{2} \theta+\left(2 \pi M_{S}^{2}-K_{i} / t_{\text {freelayer }}\right) \sin ^{2} \theta \cos ^{2} \phi$, where $K_{i}\left(\mathrm{erg} / \mathrm{cm}^{2}\right)$ is the interfacial energy between the free layer and capping and $t_{\text {freelayer }}(\mathrm{nm})$ is the thickness of the free layer. In [70], for a $2 \mathrm{~nm}$ CoFeB free layer capped with Vanadium, we see an 85\% decrease in demagnetization field. The critical current for switching is

$$
I_{c}=\frac{2 q}{\eta \hbar} \alpha \Omega H_{K} M_{S}\left[1+\frac{2 \pi M_{S}}{H_{K}}-\frac{H_{\perp}}{H_{K}}\right]
$$

where $H_{\perp}$ is the reduced demagnetization field. Thermal stability is the same as in-plane materials. The switching time required at zero temperature for initial angle, $\theta_{r m s}$, and applied current, I, is

$$
\tau^{-1}=\frac{\alpha \gamma}{\ln \left(\pi / 2 \theta_{r m s}\right)}\left(H_{K}+2 \pi M_{S}-H_{\perp}\right)\left[\frac{I}{I_{C}}-1\right]
$$

\subsection{Switching speeds of different classes of free layers}

The previous section lists the critical currents that correspond to a destabilization of the initial magnetization from their respective easy axes towards the harder axes. To actually accomplish the switching in a given time, we will need to inject a substantially larger current. The switching time depends on the interactive dynamics 
Table 4.1: A list of ferromagnetic materials that are being investigated for the free layer in STT-TAM. ॥ stands for in-plane materials while $\perp$ for perpendicular materials.

\begin{tabular}{|c|c|c|c|c|}
\hline Material & $\begin{array}{c}\text { Anisotropy } \\
\text { field, } \\
H_{K}(\mathrm{kOe})\end{array}$ & $\begin{array}{c}\text { Saturation } \\
\text { magnetization, } \\
M_{S}(\mathrm{emu} / \mathrm{cc})\end{array}$ & $\alpha$ & $\|$ or $\perp$ \\
\hline $\mathrm{TbCoFe}[57]$ & 1.2 & 139 & 0.1 & $\perp$ \\
\hline $\mathrm{CoFeB}[54,63]$ & 0.5 & 1050 & 0.01 & $\|$ \\
\hline $\mathrm{FePd}[69]$ & 33 & 1100 & 0.1 & $\perp$ \\
\hline $\mathrm{FePt}[69]$ & 116 & 1140 & 0.1 & $\perp$ \\
\hline $\begin{array}{c}\mathrm{Co}_{2} \mathrm{FeAl}{ }_{0.5} \mathrm{Si}_{0.5} \\
{[71]}\end{array}$ & 0.3 & 560 & 0.01 & $\|$ \\
\hline $\mathrm{MnAl}[69]$ & 61 & 560 & 0.01 & $\perp$ \\
\hline $\mathrm{CoPd}[61,62]$ & 10 & 450 & 0.1 & $\perp$ \\
\hline $\mathrm{NiFe}[72,73]$ & 0.25 & 800 & 0.01 & $\|$ \\
\hline $\mathrm{CoPt}[74]$ & 91 & 900 & 0.1 & $\perp$ \\
\hline $\mathrm{CoFeGe}[75]$ & 5 & 350 & 0.1 & $\perp$ \\
\hline $\mathrm{CoFeCrB}[75]$ & 0.1 & 350 & 0.01 & $\|$ \\
\hline $\mathrm{CoCrPt}[76]$ & 14.2 & 800 & 0.1 & $\perp$ \\
\hline $\mathrm{CoCrTa}[76]$ & 12.2 & 800 & 0.1 & $\perp$ \\
\hline
\end{tabular}

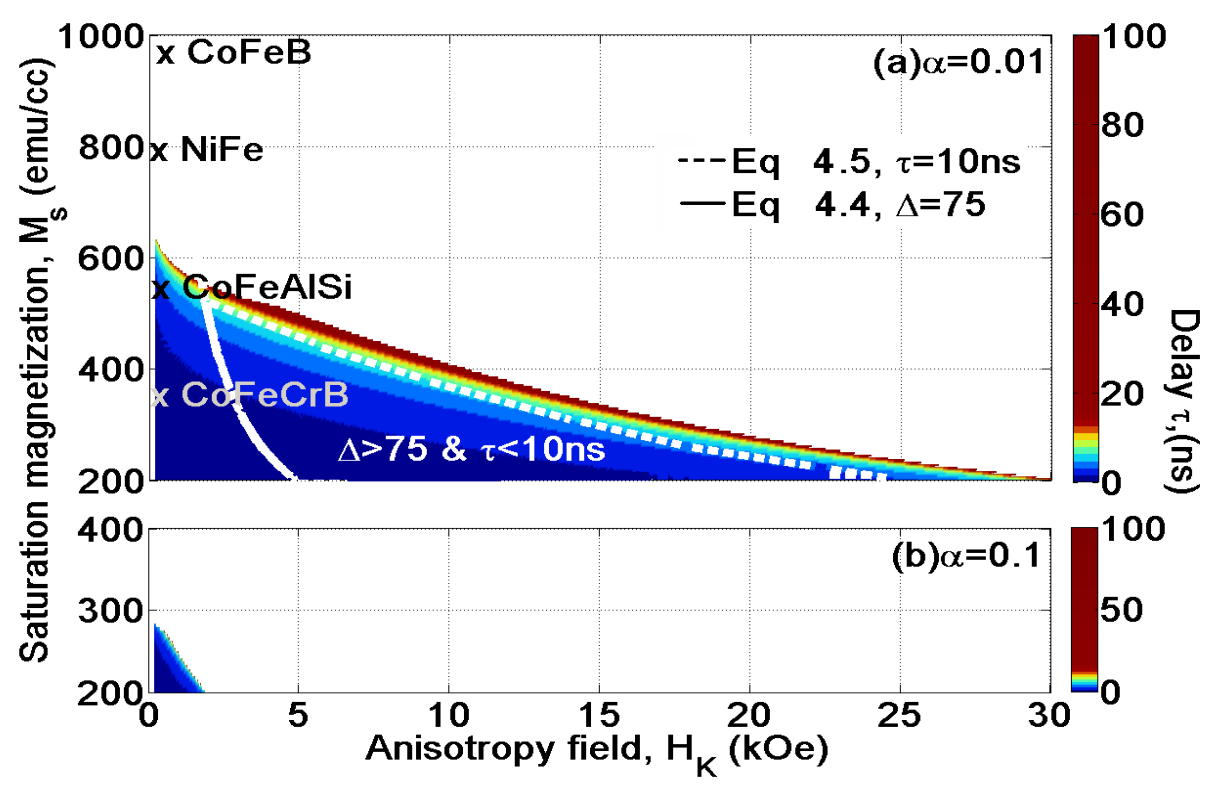

Figure 4.3: Numerically calculated switching delay (ns) for in-plane materials with varying saturation magnetization $\left(M_{S}\right)$, anisotropy field $\left(H_{K}\right)$, and Gilbert damping (a) $\alpha=0.01$ and (b) $\alpha=0.1$. A current density of $2 \mathrm{MA} / \mathrm{cm}^{2}$ is applied. Materials that switch faster, $<10$ ns with thermal stability $\Delta$ greater than 75 are preferred and are indicated by the area enclosed by the white boundaries. The white solid line indicates the boundary where $\Delta$ is 75 (using Eq 4.4, which defines a rectangular hyperbola) while the dashed line indicates the boundary where switching time is 10ns (using Eq 4.5, another rectangular hyperbola). Compared to materials with $\alpha=0.01$, materials with $\alpha=0.1$ rarely switch. At $\alpha=0.01$ and low magnetization, materials with low and high $H_{K}$ switch at the same speed, even though the energy barrier the spin current needs to overcome is proportional to $H_{K} M_{S}+2 \pi M_{S}^{2}$. The explanation for this is outlined in Fig 4.12. 


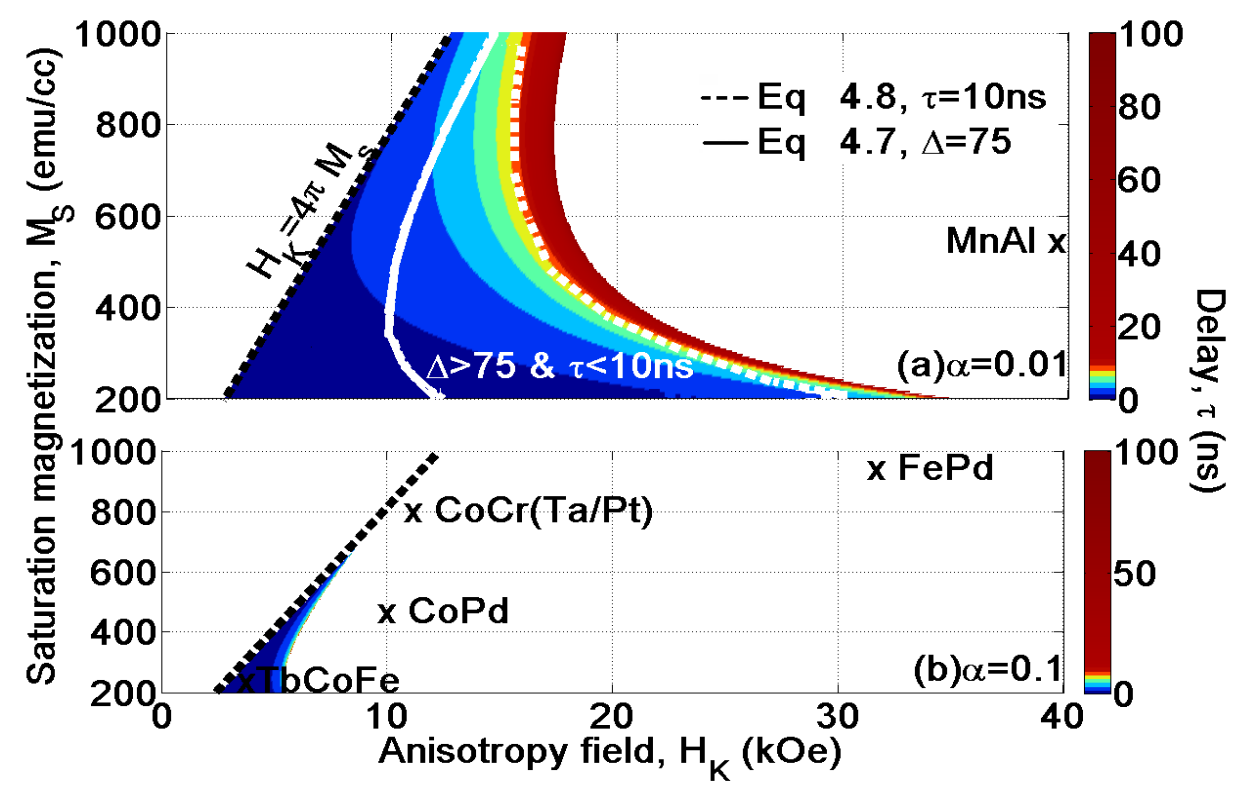

Figure 4.4: Numerically calculated switching delay (ns) for perpendicular materials with varying saturation magnetization $\left(M_{S}\right)$, anisotropy field $\left(H_{K}\right)$, and Gilbert damping : (a) $\alpha=0.01$ and (b) $\alpha=0.1$. A current density of $2 \mathrm{MA} / \mathrm{cm}^{2}$ is applied. Overall, perpendicular materials switch faster than in-plane materials, as the energy barrier the spin current needs to overcome is proportional to $H_{K} M_{S}-4 \pi M_{S}^{2}$. However, the decreased barrier has an adverse effect on thermal stability, $\Delta\left(=\left(H_{K} M_{S}-4 \pi M_{S}^{2}\right) / 2 K_{B} T\right)$. For increased $M_{S}$, thermal stability is reduced, making the range of reliable materials narrower. Most perpendicular materials have $\alpha=0.1$. Materials that switch faster $<10 \mathrm{~ns}$ and $\Delta$ greater than 75 are preferred and are indicated by the enclosed area by the white boundaries. The white solid line indicates the boundary where $\Delta$ is 75 (using Eq 4.7, which defines a rectangular hyperbola added to a linear term) while the dashed line indicates the boundary where the switching time is 10ns (using Eq 4.8). Even though higher $M_{S}$ cuts down the energy barrier, materials with high $H_{K}$ do not switch as the dominant energy barrier term is proportional to $H_{K} M_{S}$. The shape of the colored region is bounded to the left by a linear separatrix that corresponds to maintaining a perpendicular magnetization, $H_{K} M_{S}>4 \pi M_{S}^{2}$

between the various effective fields along the trajectory of the switching magnetization. Figs. 4.3, 4.4 and 4.5 show the switching delays of in-plane, perpendicular and partially-perpendicular materials with varying $M_{S}$, $H_{K}$, and $\alpha$, given a current density of $2 \mathrm{MA} / \mathrm{cm}^{2}$. The phase plots designate the switching times, while the enclosed by the white boundaries identify the range of suitable materials that successfully switch within $10 \mathrm{~ns}$ (using Eq 4.5, 4.8 and 4.10) with a $\Delta>75$ (using Eq 4.4 and 4.7). Table I lists the set of industrially relevant STT-RAM free layer materials that we study. Numerically calculated $H_{K}$ and $M_{S}$ combinations that switch within 10ns and solutions of Eq. 4.5, 4.8 and 4.10 for in-plane, perpendicular and partially-perpendicular materials are compared in Fig 4.6. Numerical and analytical results are in agreement.

We can rationalize the shapes of the phases and white polygons designating efficient switching, by outlining the various constraints they need to satisfy. In particular, we find that in-plane materials with low $M_{S}$ and low $H_{K}$ tend to switch faster, as their switching energy barrier to the spin current is proportional to $H_{K} M_{S}+2 \pi M_{S}^{2}$. Perpendicular Materials with low $M_{S}$ and low $H_{K}$ also switch faster, their barriers being 


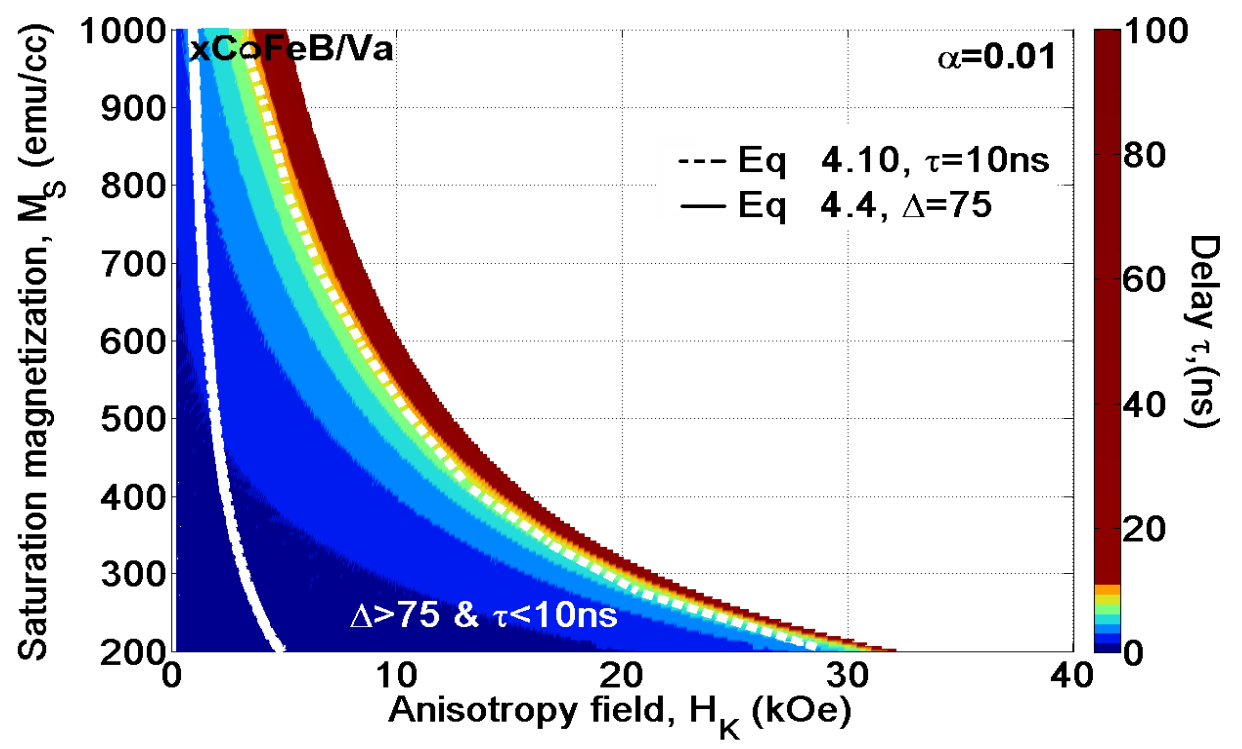

Figure 4.5: Numerically calculated switching delay (ns) for partially-perpendicular materials with varying saturation magnetization $\left(M_{S}\right)$, anisotropy field $\left(H_{K}\right)$ and $\alpha=0.01$. The demagnetization is reduced by $85 \%$ by capping. A current density of $2 \mathrm{MA} / \mathrm{cm}^{2}$ is applied. A wider range of materials (indicated by the enclosed area by the white boundaries using Eq 4.4 and 4.10) switch within 10 ns with thermal stability greater than 75 , as compared to in-plane materials with $\alpha=0.01$, generating thereby a more suitable class of free layer materials.

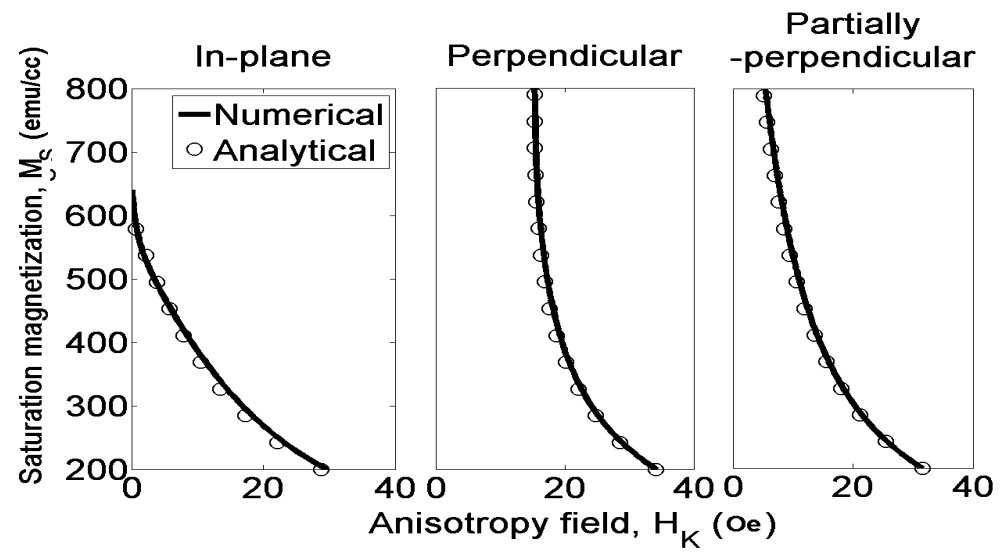

Figure 4.6: Numerically calculated $H_{K}$ and $M_{S}$ combinations that switch within 10ns, overlaid with solutions of Eqs. 4.5, 4.8 and 4.10 for in-plane, perpendicular and partially-perpendicular materials. The numerical and analytical results are in excellent agreement.

proportional to $H_{K} M_{S}-4 \pi M_{S}^{2}$. Even though a higher $M_{S}$ also cuts down the energy barrier through the negative term, materials with high $H_{K}$ do not help switching, as the dominant energy term is proportional to $H_{K} M_{S}$ 

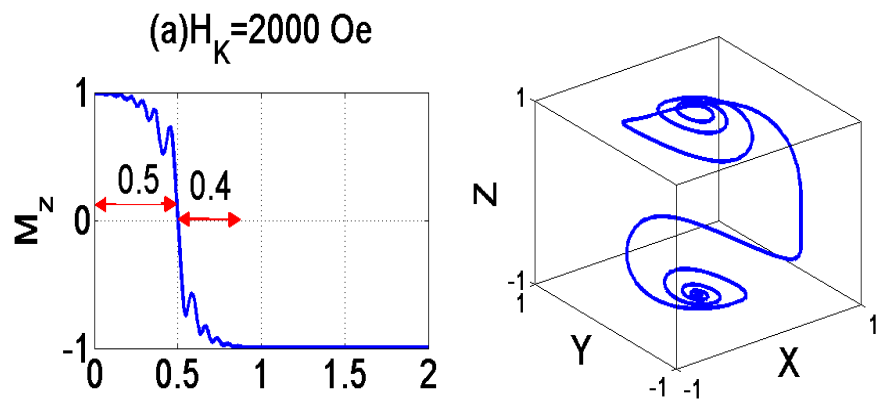

(b) $\mathrm{H}_{K}=20,000 \mathrm{Oe}$
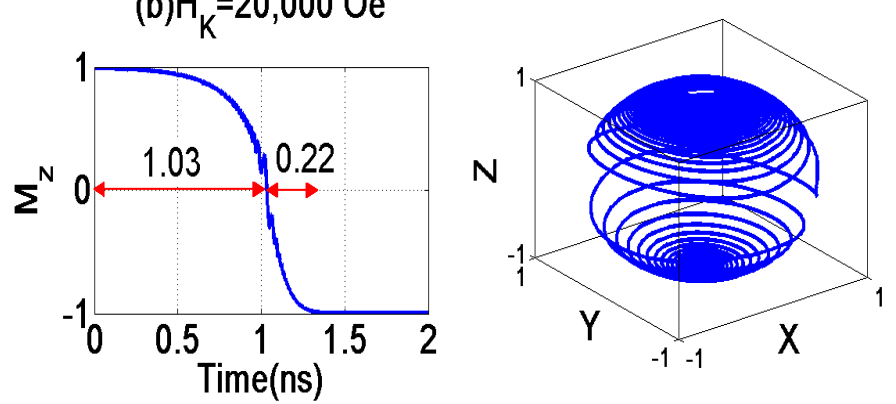

Figure 4.7: For in-plane materials with $\alpha=0.01$ and low magnetization, materials with low and high $\mathrm{H}_{K}$ switch at almost the same speed, even though the energy barrier the spin current needs to overcome is proportional to $H_{K} M_{S}+2 \pi M_{S}^{2}$. The above figure shows two cases where the saturation magnetization is fixed at (200 emu/cc), and an $\mathrm{H}_{K}$ of (a) 2000 Oe and (b) 20,000 Oe are used for the LLG simulations. At $200 \mathrm{Oe}$, the magnetization takes $0.5 \mathrm{~ns}$ to come to the equator and then another $0.4 \mathrm{~ns}$ to the south pole. At a larger $H_{K}$ of 20,000 Oe, the magnetization takes $1.03 \mathrm{~ns}$ to come to the equator but only $0.22 \mathrm{~ns}$ to come to the south pole. The asymmetry arises because the high magnetocrystalline anisotropy field hinders the magnetization while moving from the north pole to the equator, but assists it while moving from the equator to the south pole. Thus, the large time to reach the equator at higher $H_{K}$ is compensated by the faster switching from the equator to the south pole.

\subsubsection{Low vs. high $H_{K}$ in-plane materials}

For a given $M_{S}$, we find that materials with high $H_{K}$ switch almost as fast as low $H_{K}$ materials. This seems counter-intuitive, as a higher $H_{K}$ yields a higher switching barrier $\Delta$ and a correspondingly low initial angle $\theta_{r m s}$. The explanation lies in the effective magnetic fields during the first half (initial configuration to equator) vs the second half (equator to flipped configuration) of the switching process. Fig. 4.7 shows simulations with saturation magnetization set at $200 \mathrm{emu} / \mathrm{cc}$ and $H_{K}$ s et to $2000 \mathrm{Oe}$ (top) and 20,000 Oe (bottom). To isolate the field dynamics,the same spin current of $2 \mathrm{MA} / \mathrm{cm}^{2}$ is applied to the two systems, and the starting $\theta$ angle is set to $0.129 \mathrm{rad}$ in each case. At 2000 Oe, the magnetization takes 0.5 ns to come to the equator and then another $0.4 \mathrm{~ns}$ to reach the south pole. At 20,000 Oe, the magnetization takes 1.03 ns to come to the equator, but only $0.22 \mathrm{~ns}$ to come to the south pole. What is noteworthy is the unequal times taken for the two switching steps. The asymmetry arises because while the high anisotropy field hinders the magnetization from moving from the north pole to the equator, it helps while moving from the equator to the 
south pole. Therefore, the large amount of time taken to travel to the equator at high $H_{K}$ is compensated by the fast switching from the equator to the north pole.

In summary, in-plane materials with high $H_{K}$ and low $M_{S}$ would be good candidates for the free-layer. The high $H_{K}$ promotes high thermal stability, and does not compromise on switching speed because it actually helps with the switching process from the hard axis to the south pole.

\subsubsection{In-plane vs. perpendicular materials}
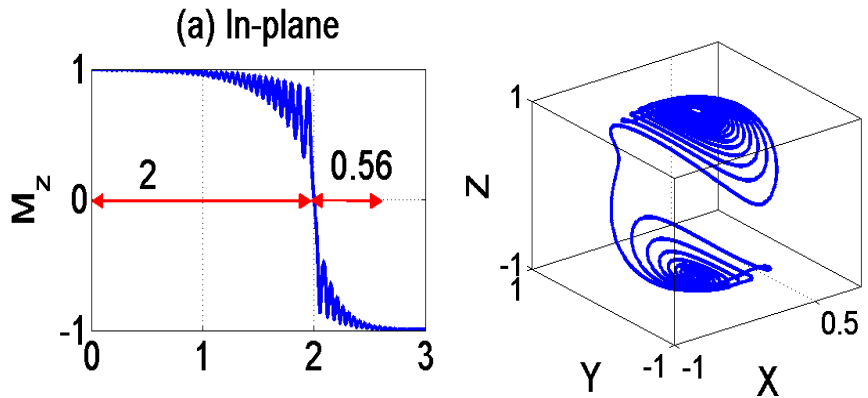

(b) Perpendicular
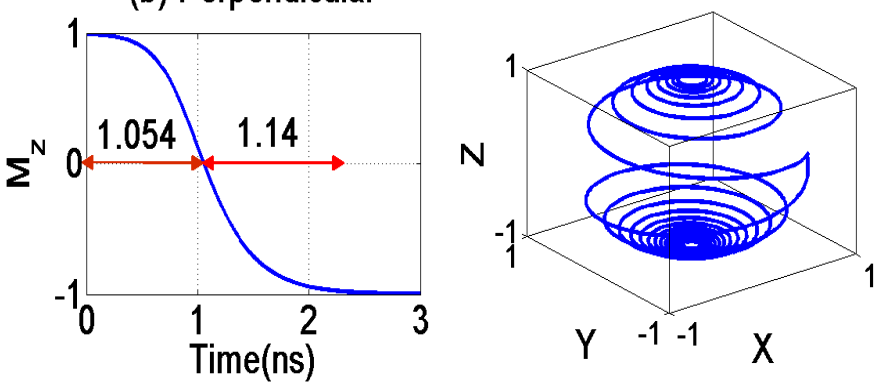

Figure 4.8: Difference between (a) in-plane and (b) perpendicular magnetization switching at $2 \mathrm{MA} / \mathrm{cm}^{2}$, $H_{K}=2000 \mathrm{Oe}, M_{S}=500 \mathrm{emu} / \mathrm{cc}$ and $\alpha=0.01$. For in-plane magnetizations, both the magnetocrystalline anisotropy and demagnetization fields hinder the magnetization during the north pole to the equator transition. After passing the equator, both fields help in moving to the south pole. For perpendicular magnetizations, the anisotropy field opposes, while the demagnetization field helps the magnetization move from north pole to equator. After passing the equator, the anisotropy helps while the demagnetization field opposes the subsequent equator to south pole switching. Thus the times taken for north pole to equator and equator to south pole transitions are comparable.

Fig. 4.8 shows the difference between in-plane and perpendicular magnetization switching at $2 \mathrm{MA} / \mathrm{cm}^{2}$, $H_{K}=2000 \mathrm{Oe}, M_{S}=500 \mathrm{emu} / \mathrm{cc}$ and $\alpha=0.01$. Recall that for in-plane magnetization switching, both the anisotropy and demagnetization fields hinder the switching from north pole to equator, and both fields help switching from equator to south pole. For perpendicular magnetization switching on the other hand, the anisotropy field opposes while the demagnetization field helps switching from north pole to equator. However, after passing the equator, the anisotropy helps in moving to the south pole, while demagnetization field 
opposes it. Thus, the times taken for the magnetization to travel from the north pole to the equator and then from the equator to the south pole are comparable.

For comparable Gilbert damping $\alpha$, a wider range of perpendicular materials switch than in-plane materials, the energy barrier being proportional to $H_{K} M_{S}-4 \pi M_{S}^{2}$ (Fig 4.8a). The steep curve to the right of the colored region at 100ns coalesces ultimately with the linear boundary to the left that corresponds to $H_{K} M_{S}>4 \pi M_{S}^{2}$, in other words, maintaining a perpendicular magnetization. The white polygon includes the additional inequality $\Delta>75$, which gives a rectangular hyperbola on the $M_{S} H_{K}$ plot. Unfortunately, most perpendicular materials known today have high Gilbert damping, making them undesirable for STT-RAM use (Fig 4.4).

Perpendicular materials with low damping constant will be good candidates for STT-RAM free layers. Their switching speeds will be comparable to in-plane, but there will be a greater probability of switching.

\subsubsection{In-plane vs. partially-perpendicular materials}

(a) In-plane
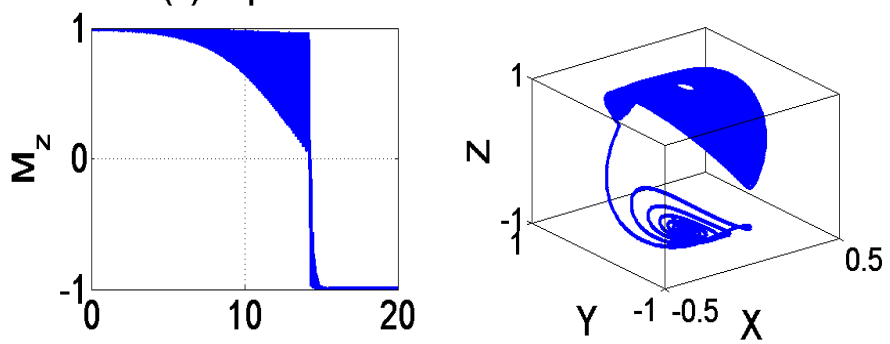

\section{(b) Partially-perpendicular}

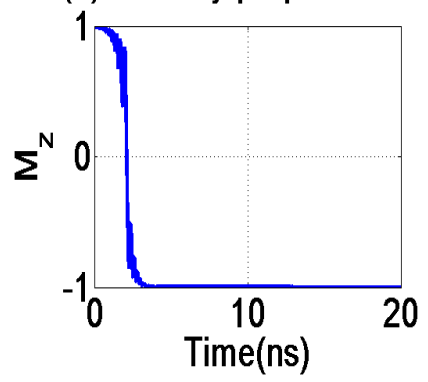

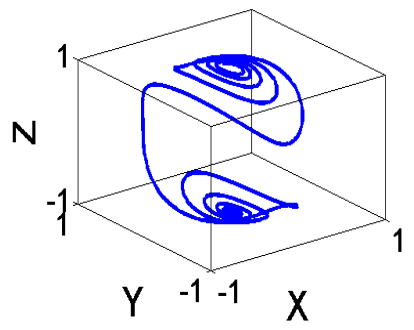

Figure 4.9: Difference between (a) in-plane and (b) partially-perpendicular magnetization switching at $2 \mathrm{MA} / \mathrm{cm}^{2}, H_{K}=2000 \mathrm{Oe}, M_{S}=500 \mathrm{emu} / \mathrm{cc}$ and $\alpha=0.01$. The decrease in the demagnetization field causes the magnetization to switch 3 times faster for the latter case.

A wider range of partially-perpendicular materials switch within $10 \mathrm{~ns}$ when compared to in-plane materials, making partially-perpendicular ultimately a more energy-efficient class of materials. Their dampings at $\alpha=0.01$ are small, yet their barriers are reduced by an overall negative term, allowing a wide range of fast 
switching events. Fig. 4.9 shows the difference between in-plane and partially-perpendicular magnetization switching at $2 \mathrm{MA} / \mathrm{cm}^{2}, H_{K}=20,000 \mathrm{Oe}, M_{S}=500 \mathrm{emu} / \mathrm{cc}$ and $\alpha=0.01$. The decrease in the demagnetization field causes the magnetization to switch 3 times faster. Capping in-plane materials with Va layer should be further investigated as their fast switching speed shows great promises for low energy switching.

\subsection{Material directions needed to be taken}

A wide variety of materials, cataloged into three magnetic classes, are explored for their switching speeds at a given thermal stability and switching current density. In order to be considered a suitable candidate for the free-layer in STT-RAM, the material needs to conform to 3 requirements: (1) high thermal stability to prevent soft error, (2) ability to switch with low applied spin current and (3) high switching speed, as the energy consumed during the write process is proportional to total time it takes to switch. In order for faster and more reliable, energy-efficient switching in the thermally stable free-layer of a STT-RAM, the following material paths should be taken: (a) In-plane materials with high $H_{K}$ and low $M_{S}$ (high $H_{K}$ increases the speed of switching, especially during the second half, and has greater thermal stability); (b) perpendicular materials with low damping - reaching comparable switching speeds with in-plane but with greater probability to switch; and (c) anti-ferromagnetically capped partially-perpendicular materials - capping with Va layer decreases the demagnetization field, which enables the switching to occur faster without compromising the thermal stability. It is worth emphasizing at this stage that the study presented here is entirely based on the magnetic properties of the contacts, as captured by LLG. A separate material phase space will correspond to the electronic properties and ultimately the voltage required to generate the drive current, namely, the contact and barrier effective masses, tunnel barrier height and width, and the polarization in the contacts[25]. These can be extracted and studied using the NEGF equation, and alternately using a modified Simmons equation. While the LLG study yields the critical current density and switching speeds, the Simmons equation would provide in addition the switching voltages, i.e., the tunnel barrier resistances required to accomplish these switching events, thereby providing the total energy dissipated during the switching event. 


\section{Chapter 5}

\section{Energy-delay-reliability trade-off in perpendicular STT-RAM}

In order to compete with existing embedded technology, an STT-RAM bit cell should be able to meet the following requirements: (i) thermal stability $(\Delta)$ must be greater than 60 to achieve high static reliability; (ii) a very low write error rate (WER) $\sim 10^{-9}$ with a fast device write time $<10$ ns; and (iii) an overall low energy write $(<1 \mathrm{pJ})$, which corresponds to a low switching voltage [24]. Achieving such a diverse set of targets will require meticulous design and material engineering.

The reliability of the writing process in arrays of STT-RAM cells is dependent on the following factors: (i) variation of the electronic, magnetic and geometrical parameters across the array [77, 78], (ii) thermally activated initial angle, $\theta_{0}$, at the beginning of the writing process, (iii) thermal fluctuations during the writing process[79] and (iv) voltage drop across the 1-transistor 1-MTJ cell[64]. For this work, we disregard the voltage drop across the transistor in (iv) as it it outside the scope of this work. We address the rest in our model. While full-fledged stochastic material and circuit simulations can help narrow down the target compositions, they are time-consuming. Therefore, it is important to develop in parallel a fast, quasi-analytical physics based model to explore the multi-parameters optimization problem involved.

We introduce a quasi-analytical model (Fig 5.1) that can be used to calculate the current and energy consumed during a write operation with an acceptable WER for a given pulse width, $\tau$, in a perpendicular STT-RAM cell. The calculation is done in three blocks: (1) Given a write-error-rate and switching time, we use an expression for switching errors from [53] to extract the $I / I_{C}$ ratio, where $I$ is the current applied and $I_{C}$ is the critical current. (2) The critical switching current $I_{C}$ is calculated using the Landau-Lifschitz-Gilbert (LLG) equation to yield the applied current $I$. This expression also includes additional effects due to 


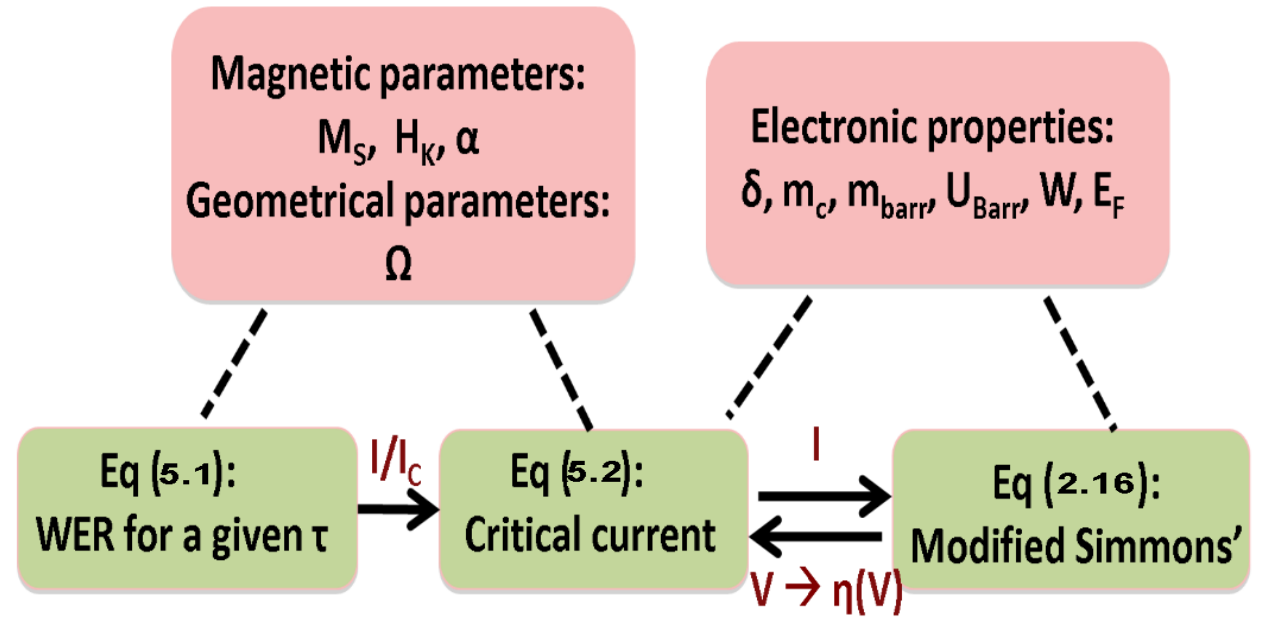

Figure 5.1: Three coupled equation blocks allow us to compute the energy consumption for a write operation in a perpendicular STT-RAM cell within a given switching time and error threshold. Equation block 1: Given a write-error-rate (WER) and delay time $\tau$, we use the non-switching expression, Eq.5.1, to extract the overdrive $I / I_{C}$ ratio, where $I$ is the current applied and $I_{C}$ is the critical switching current. Equation block 2: gives us the critical switching current $I_{C}$ using Eq. 5.2. From these two blocks, we get $I$. Equation block 3: Using a modified version of Simmons equation, Eq. 5.3, we can then estimate the switching voltage $V$ and thence the switching energy $I V \tau$. The last two steps have to be solved self-consistently, as the switching voltage for a given WER depends on current $I_{C}$, which in turn depends on the voltage-dependent polarization $\eta(V)$, which further depends on the transport parameters $E_{F}, m_{c}$ and $\delta$.

voltage-dependent polarization responsible for observed switching asymmetries[51]. (3) Using a modified version of Simmons tunneling current equation, thats includes the crucial pre-factors responsible for TMR, we can then estimate the switching voltage and thence the switching energy cost. The last two steps are coupled and need to be solved self-consistently, as the switching voltage depends on critical current, which depends on polarization, which in turn depends back on voltage. The energy consumed during the write operation can then be estimated from the switching current, voltage and time as $I V \tau$.

Coupling all three equations - the WER expression, the modified Simmons tunneling equation and the modified critical current equation[52] including the role of voltage-dependent polarization are non-standard in the literature. Note that this paper focuses on perpendicular materials, for which the WER expression has been explicitly worked out and calibrated against stochastic LLG solutions[53]. Section 5.1 addresses the effect of thermal fluctuation when the free layer is at equilibrium and when it is switching. Section 5.3 describes the worst case current needed for perpendicular STT-RAM, based on solving the Fokker-Planck equation. In section 5.4, we talk about how to calculate the voltage needed to generate the applied current using the Simmons' equation modified to include spin dependent tunneling. The modified equations are used to extract electronic parameters from published $\mathrm{CoFeB} / \mathrm{MgO} / \mathrm{CoFeB}$ MTJ current-voltage graphs. Using the parameters extracted from the fit in section 5.4, the worst case energy consumption is thereafter calculated 


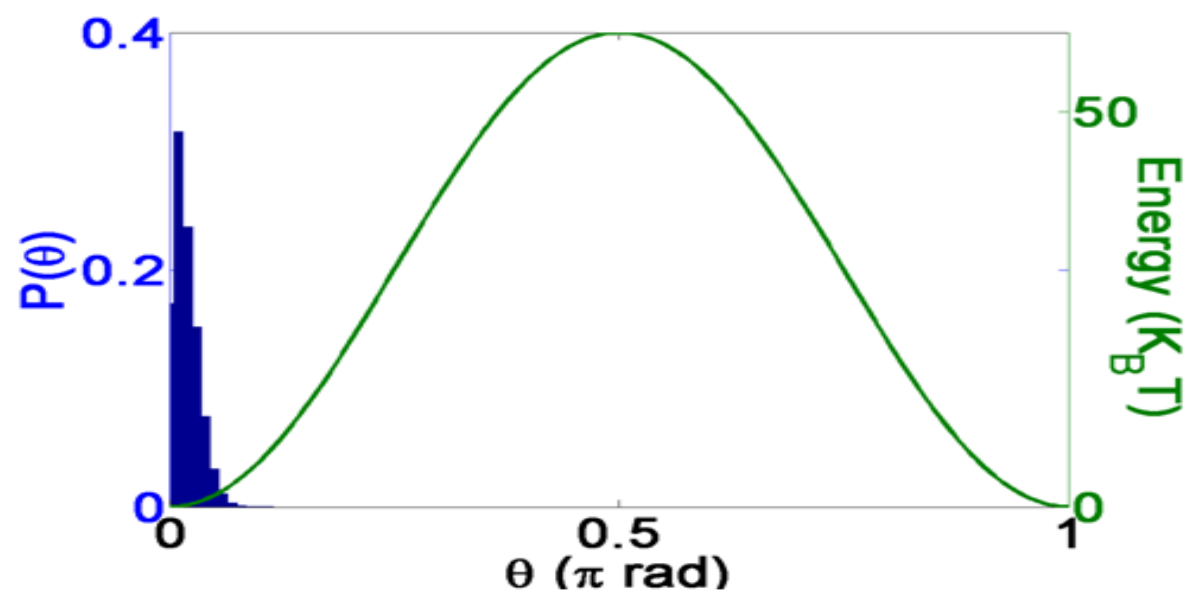

Figure 5.2: Magnetization distribution at equillibrium

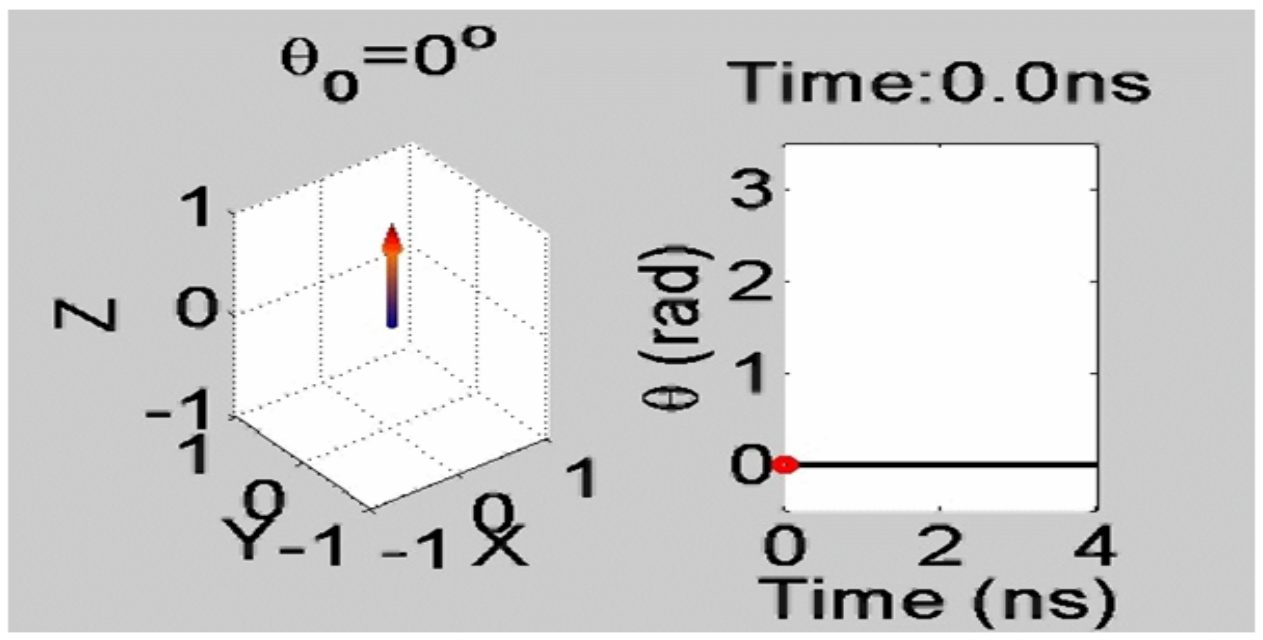

Figure 5.3: With out thermal fluctuation, the magnetization cannnot dislogde from the stagnation point and switch.

for a given $\Delta$ and error rate in section 5.5. The effect of manufacturing variations on energy consumption is also studied in section 5.6.

\subsection{Thermal fluctuation: blessing or curse ?}

Thermally activated initial angle, $\theta_{0}$, at the beginning of the writing process and thermal fluctuations during the writing process are the dominant players when it comes to the writing process in STT-RAM, with the initial angle being the most critical. The free layer in the STT-RAM MTJ memory cell is responsible for data storage, and must have enough stability against stochastic thermal switching to retain data for at least 10 years [64]. In other words, the thermal stability, $\Delta$, needs to be greater than 60 . At equilibrium stage, 


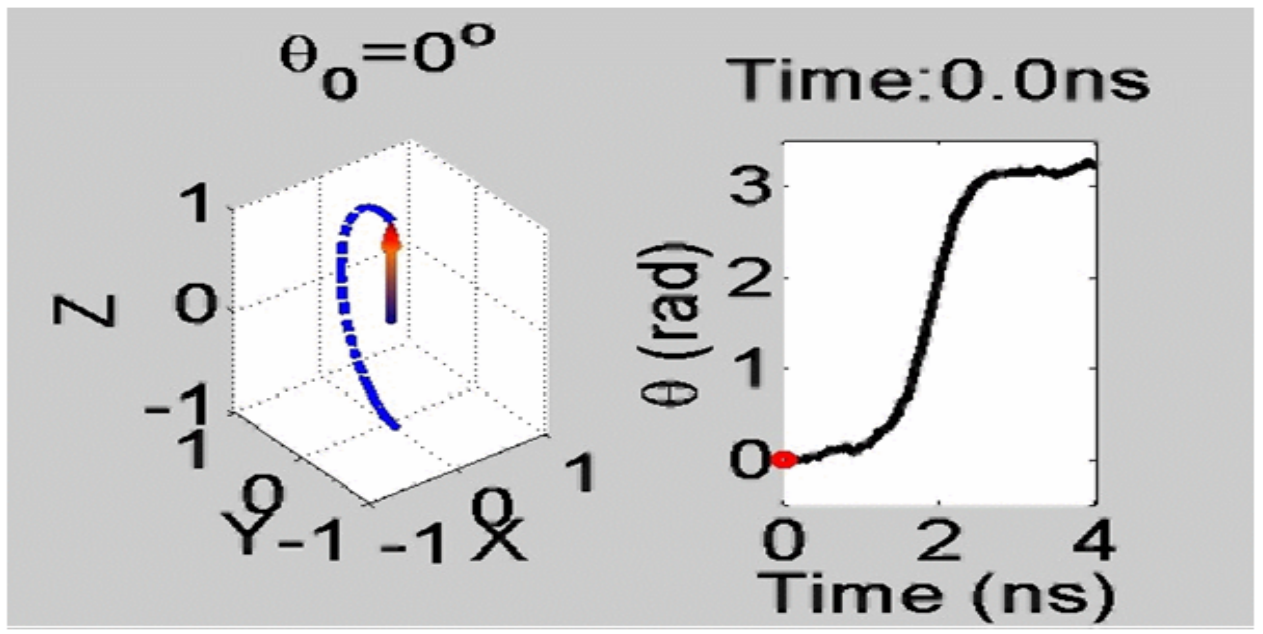

Figure 5.4: Thermal fluctuations dislodge the magnetization from the stagnation point, enabling it to switch.

the magnetization of free layer is distributed around the energy minimum (Fig. 5.2). Increased temperature would spread the distribution further, increasing the probability of suddenly switching to the other minimum energy point and losing the bit information stored in the magnetic tunnel junction. Therefore, thermal fluctuation is bad news for the static reliability of the STT-RAM device.

While we desire the device to have high thermal stability, the critical current and the time needed to switch also increase proportionally. Higher thermal stability also contributes to longer switching tails, as the magnetization tends to be nearer to the stagnation points $(\theta=0$ or $\pi)$ in the potential landscape, without any external perturbation. Near the stagnation point, the torque proportional to $\sin \theta$ is too small to initiate switching. Therefore a thermally stable free layer would require a relatively high current to have very low error rate $10^{-9}$ for switching within 5-10 ns, resulting in costlier write operations. For systems with high $\Delta$, thermal fluctuations is good news for spin-transfer torque switching. Without thermal fluctuations, the magnetization will remain at the stagnation and not switch with an applied current (Fig 5.3). Thermal fluctuation will actually help the magnetization to budge out of its stagnation point and switch (Fig 5.4).

\subsection{Current needed for worst case writing}

Given a desired write-error-rate (WER) and switching delay $\tau$, we can calculate the amount of current overdrive required relative to the critical switching current [53]. Numerically this amounts to introducing a stochastic Langevin thermal torque in LLG and then taking a time average over many runs. The equivalent probabilistic approach is to solve the Fokker-Planck equation for the probability density of each configuration and then average over the distribution to extract various moments. The Fokker-Planck equation can be solved analytically for the simple case of a perpendicular material with uniaxial anisotropy in the macrospin 


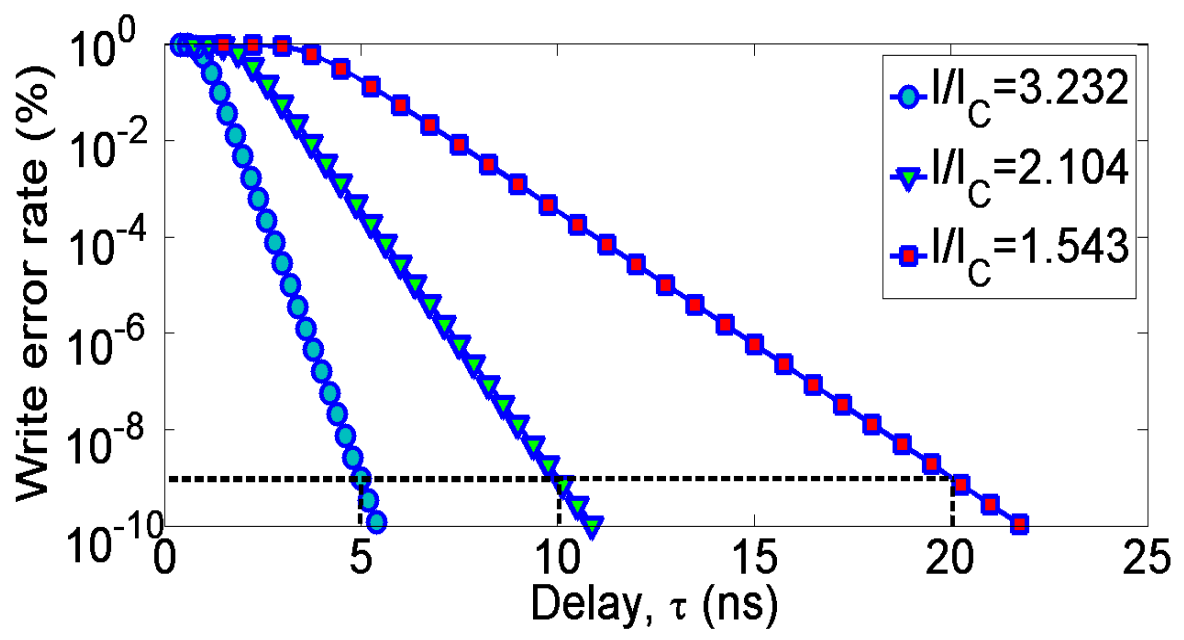

Figure 5.5: Write error rate vs. delay time at room temperature for $\Delta=60$. To attain an error rate of $10^{-9}$ within given delay times $5 \mathrm{~ns}, 10 \mathrm{~ns}$ and $20 \mathrm{~ns}$, current pulses of $3.232 I_{C}, 2.104 I_{C}$ and $1.543 I_{C}$ are needed. We use $H_{K}$ of $3.34 \mathrm{kOe}$ and $M_{S}$ of $1257.3 \mathrm{emu} / \mathrm{cc}$ for this estimate

approximation. The corresponding probability of not switching within a given delay $\tau$, defining the WER, is expressed as

$$
\begin{aligned}
W E R(\tau) & =1-\exp \left[\frac{-\pi^{2} \Delta(i-1) / 4}{i e^{2 \alpha \gamma H_{K} \tau(i-1) /\left(1+\alpha^{2}\right)}-1}\right] \\
i & =I / I_{C}
\end{aligned}
$$

where $I$ is the current required and $I_{C}$ is the critical switching current, $\alpha$ is the Gilbert damping parameter, $\gamma$ is the gyromagnetic ratio, and $\Delta=H_{K} M_{S} \Omega / 2 k_{B} T$ is the thermal stability of the free layer. $\Omega$ is the volume of the free layer and $M_{S}$ is the saturation magnetization. $H_{K}=H_{K}^{C}-4 \pi M_{S}^{2}$ is the effective anisotropy field resulting from both crystalline anisotropy $\left(H_{K}^{C}\right)$ and demagnetization field. $H_{K} M_{S} \Omega / 2$ is the energy barrier that separates the two magnetization directions, $\theta=0$ and $\pi$. Eqn. 5.1 is valid for precessional switching where $i>>1$. It is not uncommon to find stray STT-RAM cells in large arrays, which cannot operate above $i>>1$ due to manufacturing errors. Unfortunately, our model will not be able to accurately capture these stray bits. Fig. 5.5 shows write error rate vs. delay time at room temperature for $\Delta=60$. To attain an error rate of $10^{-9}$ within given delay times $5 \mathrm{~ns}, 10 \mathrm{~ns}$ and $20 \mathrm{~ns}$, current pulses of $3.232 I_{C}, 2.104 I_{C}$ and $1.543 I_{C}$ are needed. We use $H_{K}$ of $3.34 \mathrm{kOe}$ and $M_{S}$ of $1257.3 \mathrm{emu} / \mathrm{cc}$ for this estimate [58].

Solving the LLG equation we get a critical current similar to the expression derived by J.Z. Sun[52], but modified to include the proper switching asymmetry through the effective voltage dependent polarization(described in the next section),

$$
I_{C}=2 \alpha e H_{K} M_{S} \Omega / \hbar \eta(V)
$$



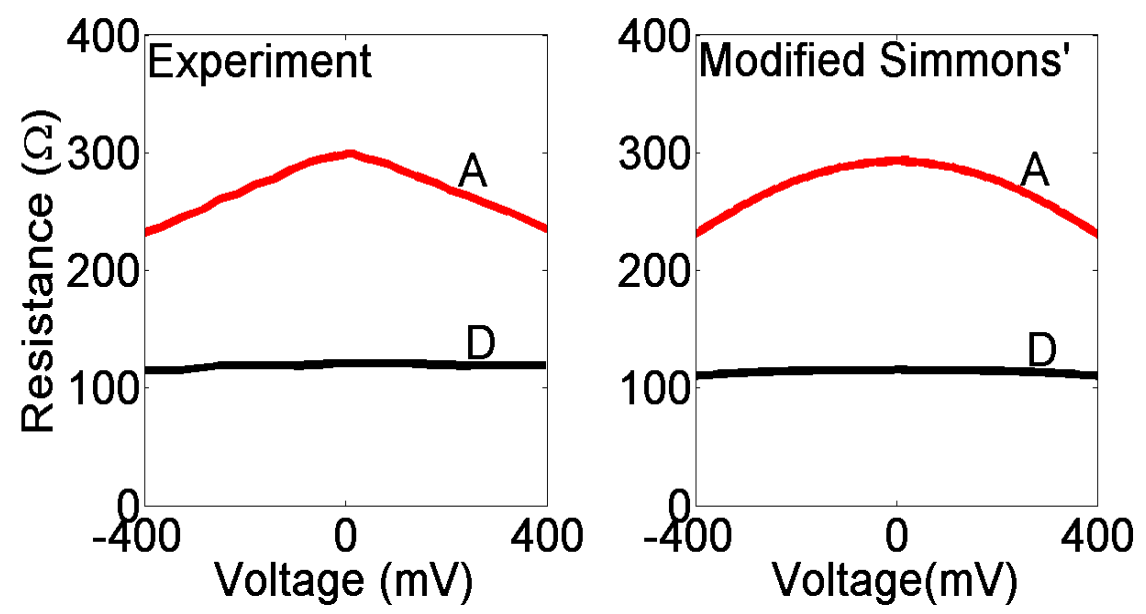

Figure 5.6: Parameter extraction from published CoFeB/MgO/CoFeB MTJ resistance-voltage curve (H. Kuboto et al, "Quantitative measurement of voltage dependence of spin-transfer torque in Mg0-based magnetic tunnel junctions with modified Simmons' mode",2001)l. The parameters extracted from the model are $E_{F}$ $=2.2 \mathrm{eV}, U=1 \mathrm{eV}, \mathrm{W}=1 \mathrm{~nm}, m_{c}=0.3 m_{0}, m_{\text {barr }}=0.18 m_{0}$ and $\delta=1.98 \mathrm{eV}$. Curve A is for anti-parallel mode while $\mathrm{D}$ for parallel mode. $m_{0}$ is the mass of an electron.

From the given $W E R$ and $\tau$, we can get the current overdrive $i=I / I_{C}$. In conjunction with the critical current above, we can then extract the applied current $I$. Finally, using the modified version of Simmons equation described in the next section, we can then estimate the switching voltage.

\subsection{Voltage needed for worst case writing}

Section 2.2.2 show a quasi-analytical modified Simmons' equation that calculates spin dependent tunneling in a magnetic tunnel junction. Fig. 5.6 show results from the analytical model fitted with a published

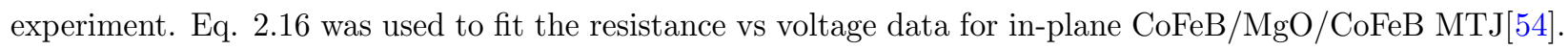
The parameters extracted from the model are listed in the figure caption. Given an applied current, the voltage needed to generate that amount of current that be numerically back calculated from the equation.

\subsection{Worst case energy consumption and switching delay due to thermal fluctuations}

Using the self-consistent analytical model illustrated in Fig. 5.1, we calculate the worst case energy consumption during a write operation at room temperature for $\Delta=60$ and various delays (Fig. 5.7) due to thermal fluctuations. Energy consumption during P to AP switching is greater than that consumed during AP to $\mathrm{P}$ switching. This is because the effective polarization (explained in chapter 3 ) of the torque at negative 

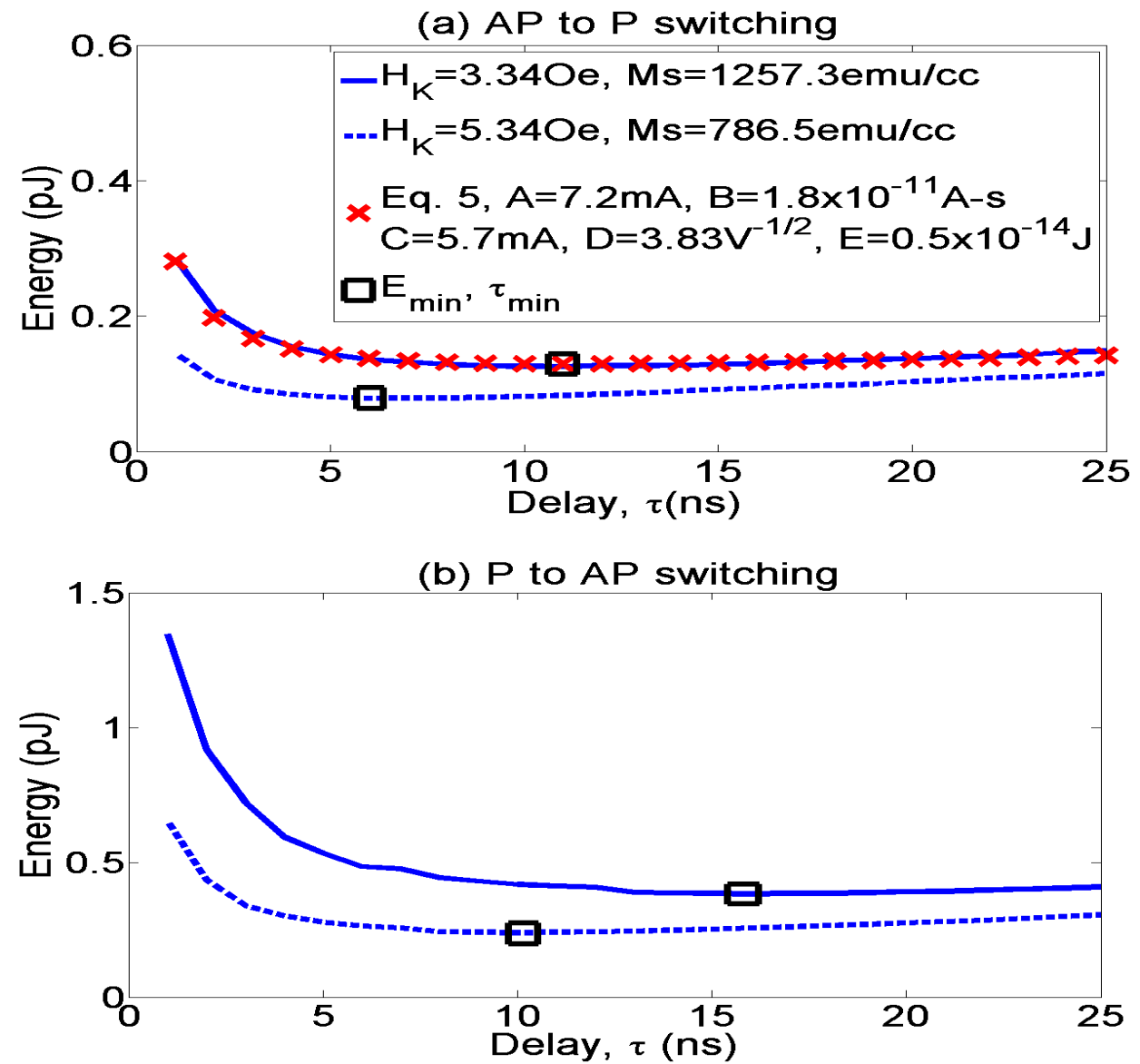

Figure 5.7: Worst case energy consumption during a write operation at room temperature for $\Delta=60$ and error rate of $10^{-9}$. Energy consumption during (a) AP to P switching is less than that consumed during (b) $\mathrm{P}$ to AP switching. This is because the effective polarization of the torque at negative voltage is less than that at positive voltage. Energy consumed is the product of current, voltage applied and delay ( $I V \tau)$. At lower delay, the current, and hence energy, decreases logarithmically with delay. At higher delay, the energy consumption increases linearly with delay.

voltage is less than that at positive voltage[51, 65]. However, for a magnetic tunnel junction with half-metallic contacts $(\delta=3 \mathrm{eV})$, energy consumption during AP to $\mathrm{P}$ switching is equal to that during $\mathrm{P}$ to AP switching because the effective polarization of the torque at negative voltage is same as that at positive voltage (Fig. $5.8)$.

Energy consumed is the product of current, voltage applied and delay $(I V \tau)$. The voltage drop considered here is the one across the MTJ. The additional voltage drop in the transistor which usually accompanies the STT-RAM cell is disregarded in this work. When the applied current is greater than the critical current, the switching is precessional and the switching delay of the free layer is inversely proportional to the current, $I \propto I_{C}+I_{C}\left(1+\alpha^{2}\right) \ln \left(\pi / 2 \theta_{r m s}\right) /\left(\alpha \gamma H_{K} \tau\right) \propto(A \tau+B)[80,53]$. Assuming that the current in the MTJ grows exponentially with applied voltage (Eq. 3), $V \propto[\ln (I / C) / D]^{2}$. The energy consumption with respect to 


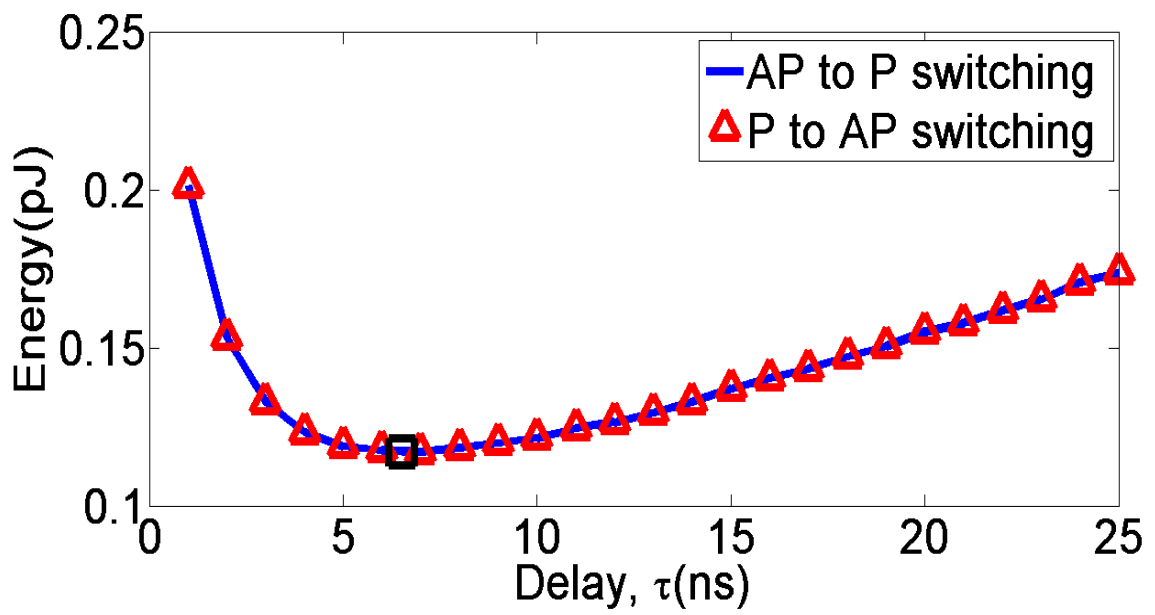

Figure 5.8: Worst case energy consumption during a write operation at room temperature for $\Delta=60$ and error rate of $10^{-9}$ for a magnetic tunnel junction with half-metallic contacts $(\delta=3 \mathrm{eV})$. Energy consumption during $\mathrm{AP}$ to $\mathrm{P}$ switching is equal to that during $\mathrm{P}$ to $\mathrm{AP}$ switching because the effective polarization of the torque at negative voltage is same as that at positive voltage. $H_{K}=5.34 \mathrm{kOe}$ and $M_{S}=786.5 \mathrm{emu} / \mathrm{cc}$.

delay is

$$
\text { Energy } \propto I V \tau \propto(A \tau+B)\left[\frac{\ln \left(\frac{A}{C}+\frac{B}{C \tau}\right)}{D}\right]^{2}+E
$$

where $\mathrm{A}\left(=I_{C}\right), \mathrm{B}\left(=I_{C}\left(1+\alpha^{2}\right) \ln \left(\pi / 2 \theta_{r m s}\right) /\left(\alpha \gamma H_{K}\right)\right), \mathrm{C}\left(=A r e a \cdot q^{2} / 4 \pi^{2} \hbar W^{2}\right), \mathrm{D}\left(=2 W \sqrt{2 m_{c}} / \hbar\right)$ and $\mathrm{E}$ are constants. $\theta_{r m s}$ is the root-mean-square initial angle of the free layer nanomagnets under thermal perturbation, calculated by the equipartition theorem, $\sin ^{2}\left(\theta_{r m s}\right)=1 / 2 \Delta$. The units of constants A, B, C and E are Amp, Amp-sec, Amp, $V^{-1 / 2}$ and J respectively. At lower delay, the energy decreases logarithmically with delay as $[\ln (I / C) / D]^{2}$ is the dominant term. At higher delay, the energy consumption increases linearly with delay as $A \tau$ is the dominant term. There is thus an energy minimum, $E_{m i n}$, corresponding to an optimal delay value, $\tau_{\text {min }}$. Fig. 5.7 shows a fit of the numerical results with Eq. 2.3. The constants to fit the energy-delay curve for $\mathrm{AP}$ to $\mathrm{P}$ switching at $H_{K}=3.34 k O e$ were: $\mathrm{A}=7.2 \mathrm{~mA}, \mathrm{~B}=1.8 \times 10^{-11} \mathrm{~A}-\mathrm{s}, \mathrm{C}=5.7 \mathrm{~mA}, \mathrm{D}=3.83 V^{-1 / 2}$ and $\mathrm{D}=0.5 \times 10^{-14} \mathrm{~J}$.

In addition, keeping $\Delta$ at 60 , if $H_{K}$ is increased from $3.34 \mathrm{kOe}$ to $5.34 \mathrm{kOe}$, and $M_{S}$ adjusted accordingly from $1257.3 \mathrm{emu} / \mathrm{cc}$ to $786.5 \mathrm{emu} / \mathrm{cc}$, the $E_{\min }$ is shifted from $0.1252 \mathrm{pJ}$ at $10.5 \mathrm{~ns}$ to $0.0783 \mathrm{pJ}$ at $6.6 \mathrm{~ns}$ for AP to $\mathrm{P}$ switching. Similarly for P to AP switching, the energy minimum shifts lower from $0.3829 \mathrm{pJ}$ at $16.25 \mathrm{~ns}$ to $0.2393 \mathrm{pJ}$ at $10 \mathrm{~ns}$. In other words, the $E_{\text {min }}$ can be reduced quite significantly by increasing the anisotropy field, $H_{K}$ and decreasing the saturation magnetization, $M_{S}$ accordingly, while maintaining thermal stability. The duration of the voltage pulse needed to achieve the switching with $10^{-9}$ write error rate, $\tau_{m i n}$, also decreases with higher anisotropy field (Fig. 5.8). 


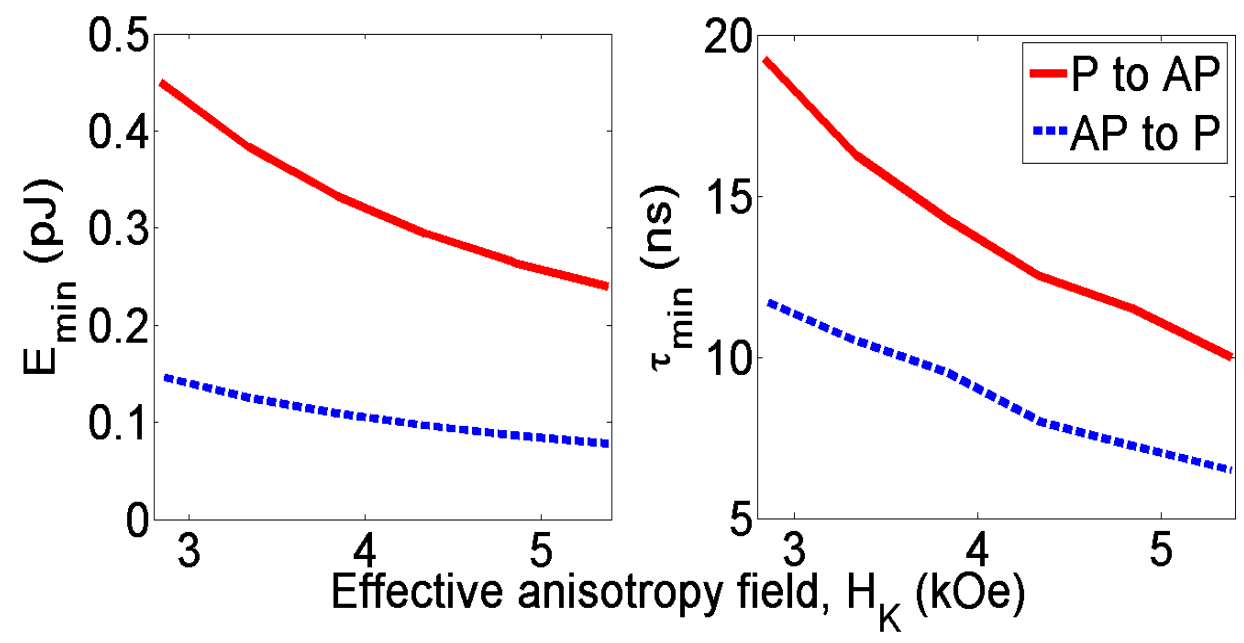

Figure 5.9: Keeping volume and thermal stability of the free layer the same $(\Delta=60)$, the minimum energy consumption can be reduced by increased the anisotropy field, $H_{K}$ and decreasing the saturation magnetization, $M_{S}$ accordingly. The duration of the voltage pulse needed to achieve the switching with $10^{-9}$ error rate also decreases with higher anisotropy field $\alpha=0.02$.

\subsection{Manufacturing variations}

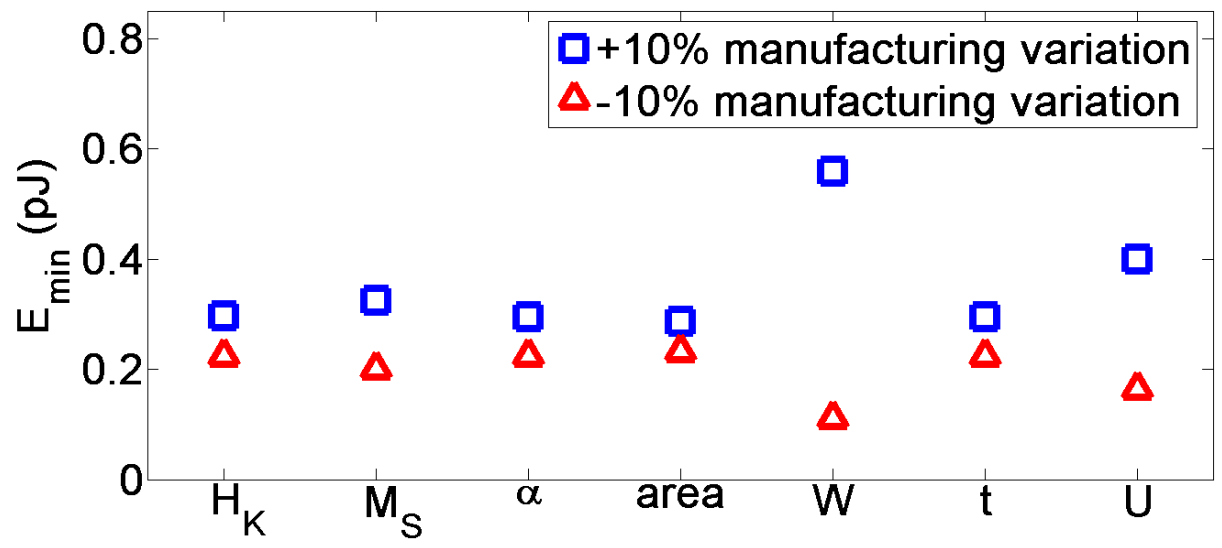

Figure 5.10: Effect of manufacturing variations on minimum energy consumption, $E_{m i n}$, during a $\mathrm{P}$ to AP write operation with an error threshold of $10^{-9}$. The varying parameters are effective anisotropy field $\left(H_{K}\right)$, saturation magnetization $\left(M_{S}\right)$, Gilbert damping parameter $(\alpha)$, cross-sectional area of the MTJ, thickness of the free layer (t), width of the tunneling barrier $(\mathrm{W})$, and ferromagnet-oxide interface (U). While variation in the thickness of the tunneling barrier has the most effect on the delay, cross-sectional area has the least. Mean value of the MTJ parameters used are $H_{K}=5.34 O e, M_{S}=785.6 \mathrm{emu} / \mathrm{cc}, \alpha=0.02$, radius of the free layer $=20 \mathrm{~nm}, \mathrm{t}=2 \mathrm{~nm}, \mathrm{~W}=1 \mathrm{~nm}$ and $\mathrm{U}=1 \mathrm{eV}$.

All the above simulation results (Figs. 5.7-5.9) only considered thermally activated initial angles, $\theta_{0}$, at the beginning of the writing process and thermal fluctuations during the writing process in a given MTJ cell with a fixed dimension, electronic and magnetic parameters. However, individual MTJ cells in STT-RAM arrays will not all have the same physical dimension or parameters because of manufacturing 


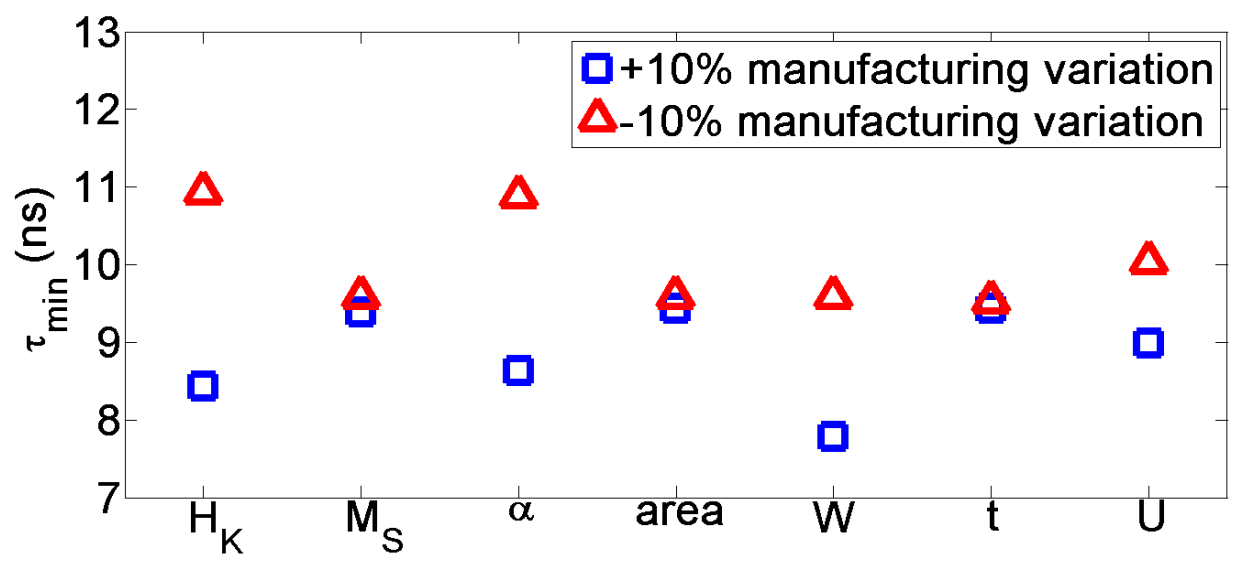

Figure 5.11: Corresponding minimum switching delay during a $\mathrm{P}$ to $\mathrm{AP}$ write operation with an error threshold of $10^{-9}$. The varying parameters are effective anisotropy field $\left(H_{K}\right)$, saturation magnetization $\left(M_{S}\right)$, Gilbert damping parameter $(\alpha)$, cross-sectional area of the MTJ, thickness of the free layer (t), width of the tunneling barrier $(\mathrm{W})$, and ferromagnet-oxide interface $(\mathrm{U})$.

variations. The key parameters, $H_{K}, M_{S}, \alpha$, cross-sectional area of the MTJ, thickness of the free layer (t), $\mathrm{W}$ and $\mathrm{U}$ can vary by $10 \%$ from their mean value[77]. The self-consistent analytical model illustrated in Fig. 5.1 can be used to evaluate the write performance against various intrinsic variabilities as show in Figs. 5.10 and 5.11. All the above parameters were varied individually by $\pm 10 \%$ and their effect on the $E_{\min }$ and $\tau_{\min }$ are reported. The error rate is fixed at $10^{-9}$. Differing $H_{K}, M_{S}$, t and cross-sectional area will affect $\Delta$. Increased $\Delta$ and $\alpha$ increases the critical current and energy consumption. Increasing barrier width and height results in more voltage being needed to get the desired current. While variation in the thickness of the tunneling barrier has the most effect, cross-sectional area has the least. The worst-case energy consumption and delay in an STT-RAM array can be calculated by considering an MTJ cell with biggest variation $(+10 \%)$ in all the parameters above. The values are $1.03 \mathrm{pJ}$ at $5.25 \mathrm{~ns}$ for $\mathrm{P}$ to AP writing. A detailed study of the manufacturing variations will be a future effort.

In this chapter, we present a quasi-analytical model that approximately calculates the energy consumed during a write operation that ensures a certain error rate and delay time. The combination of the two models enables us to calculate the energy consumption without performing a full-fledged stochastic calculation and to evaluate ways to reduce the energy consumption during worst-case writing in STT-RAM. At lower delay, the energy consumption decreases logarithmically with delay while at higher delay, it increases linearly with delay, creating a delay point where the energy consumption is minimum (Fig. 5.7). We also quantified how increasing the anisotropy field $H_{K}$ and lowering the saturation magnetization $M_{S}$ while keeping $\Delta$ fixed, can significantly reduce the energy consumption and the switching delay. The effect of the variations of geometric, magnetic and electronic properties in STT-RAM arrays is studied and it is seen that variation in the thickness of the oxide has the biggest role in determining the worst case energy consumption scenario. 


\section{Chapter 6}

\section{Energy-delay-reliability in}

\section{nanomagnetic logic using multiferroics}

While the inherent energy advantages of multiferroics has been discussed in the literature [13], there are several other issues that need to be explored, including speed of operation and write error rates. In this work, we will focus on efficient information transmission between 2 NMs in a binary wire, taking thermal perturbations into account [81]. Instead of simulating the entire binary wire, we will simulate the interplay between 2 NMs (Fig. 6.1), focusing on the unidirectional propagation of information from NM1 to NM2.

The specific design we will simulate here consists of a synthetic multiferroic stack with three layers - a magnetostrictive (Terfenol-D) and a piezoelectric (PZT) and a hard magnetic layer (Fig. 6.1a). The bistable configuration along the easy (in-plane major) axis of the Terfenol-D layer encodes logic bits ' 0 ' and ' 1 ' ( $\phi=$ $\pi / 2$ and $3 \pi / 2$ respectively)[23]. The magnets are prepared for information propagation with a small local voltage applied to a coupled piezoelectric element (PZT), stressing the magnets and switching them onto their hard (in-plane minor) axes [15]. The computation is initialized with a spin polarized current from the hard magnetic layer on the first multiferroic stack, and writing a new bit to its Terfenol-D layer using spin-transfer torque (Fig. 6.1b). Through a subsequent 'hold and release' sequence applied pairwise, two neighboring magnetic bits are 'erased' onto their hard axes, and then one is released to receive information from its prior, information carrying neighbor. Since only one neighbor has a bit information written on it, the information propagates unidirectionally.

The major axis of NM1 is increased to account for the dipolar coupling to its left neighbors. Fig. 6.2a shows the 3-step information transmission process. In step A, information has just been written into NM1, while the magnetization of NM2 is still parallel to it. In step B, a small local voltage applied to the piezoelectric 


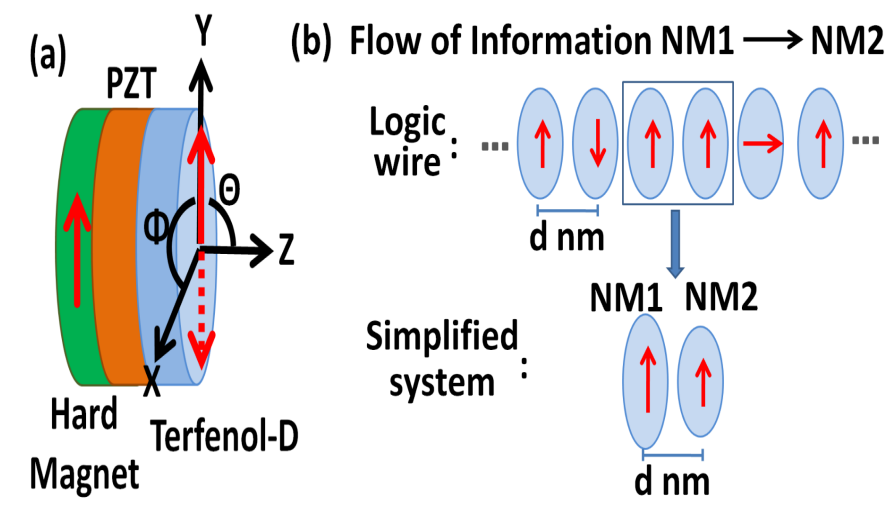

Figure 6.1: A binary wire, spaced $\mathrm{d}$ nm apart. A longer wire is simplified to a 2 NMs system (NM1 and NM2). The major axis of NM1 is increased to account for the dipolar coupling to its left neighbors.

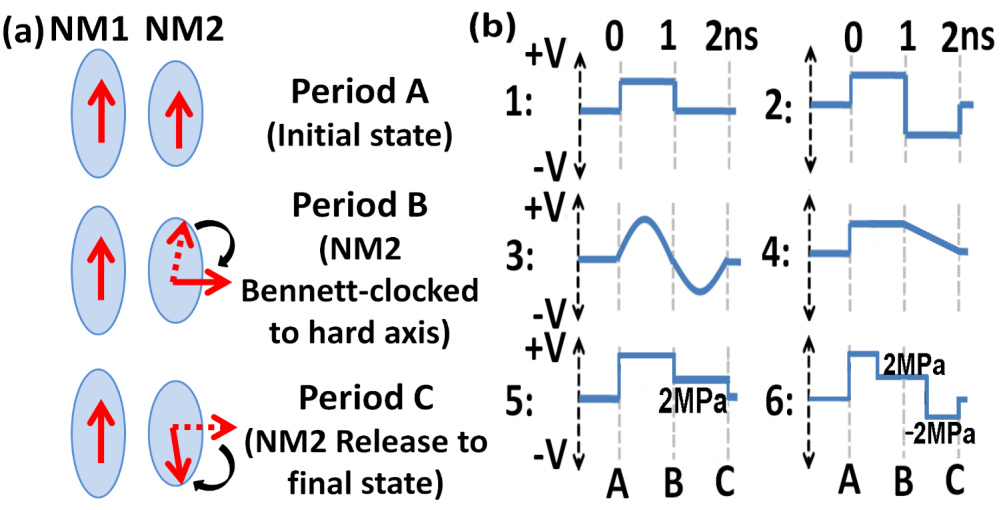

Figure 6.2: (a) 3-step information transmission process in 2 NMs. (b) Stress profiles with abrupt removal: Cases 1 and 2, tapered: Cases 3 and 4 and reduced to Region II (to operate in an energy landscape where stress cancels out the demagnetization field and only dipolar interaction with NM1 exists): Cases 5 and 6 . The negative stress in 2, 3 and 6 allow faster switching.

element strains the magnetostrictive layer of NM2, causing its magnetization to switch to its hard axis. In Period C, NM2 is released by withdrawing the stress, causing it to relax to the easy axis, with its final orientation anti-parallel to NM1 dictated solely by its dipolar coupling [21].

At the level of 2 NMs itself, we can see the role of switching reliability. While a $100 \%$ success in switching between the 2 NMs proceeds unchanged, a slightly lower success rate of $99 \%$ translates to only a $56.63 \%$ success rate after information transmission trough 100 NMs (assuming odd number of NMs failures in the system). The success rate for the switching of NM2 depends on two factors: (i) we need a high enough stress in Period B to dislodge any spins stuck at the stagnation points $(\phi=\pi / 2$ or $3 \pi / 2)$ and destabilize them towards the hard axis ( $\phi=0$ or $\pi$ ), and (ii) we also need prominent dipolar coupling in periods B and C when the magnetization is near the hard axis, so that its final orientation upon stress release can be correctly determined by the previous neighbor, avoiding any back-hopping towards the starting point. The stressing profile for NM2 has to be designed with the above two facts in mind to minimize the error-delay product at a 
modest energy cost. The aim of this chapter is to identify such a profile.

In section 6.2, an overview of the energy landscape of the $2 \mathrm{NMs}$ is given. The critical stress to rotate NM2 is calculated, along with the minimum spacing between NMs while still retaining their ground state along the easy axes, antiparallel to each other. A regime is identified just above the critical stress where the applied stress cancels out the demagnetization field and the only profile in the energy landscape is that of the dipolar coupling with NM1. Switching in this 'dipole-dominated' regime will guarantee a high success rate, albeit at a very slow speed. On the other hand, stress levels much above the dipole dominated region ensure high speed switching but erases the advantage of dipole directionality.

In section 6.3, we will explore the switching profiles outlined in Fig. 6.2b, and study their reliability, delay and energy consumption. Our results can be understood in the light of the aforementioned trade-off between high stress vs dipole dominated regimes. Case 1 has a voltage pulse of 1 ns. To switch faster, a negative stress is applied in Case 2, at the end of Period B. However, the faster switching comes with lower reliability and higher energy consumption. Case 3 is a very low-power, sinusoidal stress profile with a period of 2 ns. Stress is removed slowly at the end of Period B and a negative stress during Period C switches the magnetization of NM2 faster. The tapered stress removal helps with reliability as the stress operates in dipole dominated region for a brief period of time. However, the negative swing in the stress profile negates the achieved higher reliability. In Case 4, after keeping the stress on for 1ns, it's slowly removed in 1 ns instead of abrupt removal, achieving higher reliability than Cases 1, 2, and 3. In Case 5, after keeping the stress high for 1ns, it is reduced to $2 \mathrm{MPa}$ for $1 \mathrm{~ns}$, enabling the magnetization to operate in dipole dominated region for sometime as discussed in section 6.2. Case 6 is similar to 5 , with an additional negative stress of $-2 \mathrm{MPa}$ in the end to speed up switching delay. Cases 5 and 6 both have very high reliability. It is found that Case 6 excels in both reliable switching and delay, with a marginally higher energy consumption than Case 5.

\subsection{Energy landscape of two dipole coupled nanomagnets (NM1 and NM2)}

We will first describe the dynamical equations of the nanomagnets under stress. The elliptical Terfonal-D NM2 has an inhomogeneous magnetization $\vec{M}$ with major axis, a $=101.75 \mathrm{~nm}$, minor axis, $\mathrm{b}=98.25 \mathrm{~nm}$ and thickness, $\mathrm{t}=10 \mathrm{~nm}$. The Terfonal-D layer is coupled elastically with 40nm thick PZT disk[16, 13]. For a this dimension, the exchange coupling penalty prevents the formation of multi-domain states, so that we can ignore the spatial variation of magnetization and model it as a single-domain NM using macrospin dynamics[82]. The variation in the magnetization of any single-domain multiferroic NM with time, under the 
influence of an effective magnetic field $\vec{H}_{e f f}$ is described by the Landau-Lifshitz-Gilbert (LLG) equation $[83]$

$$
\frac{d \vec{M}}{d t}=-\gamma \vec{M} \times \vec{H}_{e f f}-\frac{\alpha \gamma}{M_{S}}\left[\vec{M} \times\left(\vec{M} \times \vec{H}_{e f f}\right)\right]
$$

where $\alpha$ is the Gilbert damping constant, $\gamma$ is the Gyromagnetic ratio and $M_{S}$ is the saturation magnetization. $\vec{H}_{e f f}$ is the effective magnetic field on any one multiferroic element, which is the gradient of the total energy of that element with respect to its magnetization vector.

$$
\vec{H}_{e f f}=-\frac{1}{\mu_{0} \Omega} \frac{\delta E}{\delta \vec{M}}
$$

Here $\mu_{0}$ is the vacuum permeability and $\mathrm{E}$ is the total free energy of the multiferroic element of volume $\Omega(=\pi a b t / 4)$. The total free energy of a NM at $0 \mathrm{~K}$ temperature is given by

$$
E=E_{\text {demag }}+E_{\text {stress }}+E_{\text {dipole }}
$$

where $E_{\text {demag }}$ is the shape anisotropy energy due to the elliptical shape of the NM, $E_{\text {stress }}$ is the stress anisotropy energy caused by the stress transferred to the magnetostrictive layer of the multiferroic upon application of an electrostatic potential to the piezoelectric layer and $E_{\text {dipole }}$ is the dipole-dipole interaction energy between the NMs. The demagnetization energy of the $\mathrm{i}^{\text {th }} \mathrm{NM}$ in spherical coordinates, $\theta_{i}$ and $\phi_{i}$, is

$$
E_{\text {demag }}=\frac{\mu_{0} M_{S}^{2} \Omega}{2}\left(N d_{x x} \sin ^{2} \theta_{i} \cos ^{2} \phi_{i}+N d_{y y} \sin ^{2} \theta_{i} \sin ^{2} \phi_{i}+N d_{z z} \cos ^{2} \theta_{i}\right)
$$

where $N d_{x x}, N d_{y y}$ and $N d_{z z}$ are respectively the demagnetization factors along the x, y and z directions and are dependent on $\mathrm{a}, \mathrm{b}$ and $\mathrm{t}$. The equations to calculate the demagnetization factors can be found in $[84,16]$. The stress anisotropy energy in the $\mathrm{i}^{\text {th }}$ element due to a stress applied along its major axis is

$$
E_{\text {stress }}=-\frac{3}{2} \lambda \sigma \Omega \sin ^{2} \theta_{i} \sin ^{2} \phi_{i}
$$

where $(3 / 2) \lambda$ is the is the saturation magnetostriction and $\sigma$ is the applied stress. $\sigma$ is negative for compression and positive for tension[16]. The dipole-dipole interaction energy with $\mathrm{j}$ th NM is

$$
E_{\text {dipole }}=\frac{\mu_{0} M_{S}^{2} \Omega_{i} \Omega_{j}}{4 \pi R^{3}}\left[-2\left(\sin \theta_{i} \cos \phi_{i}\right)\left(\sin \theta_{j} \cos \phi_{j}\right)+\left(\sin \theta_{i} \sin \phi_{i}\right)\left(\sin \theta_{j} \sin \phi_{j}\right)+\cos \theta_{i} \cos \theta_{j}\right]
$$

where $\mathrm{R}$ is the separation between their centers. 

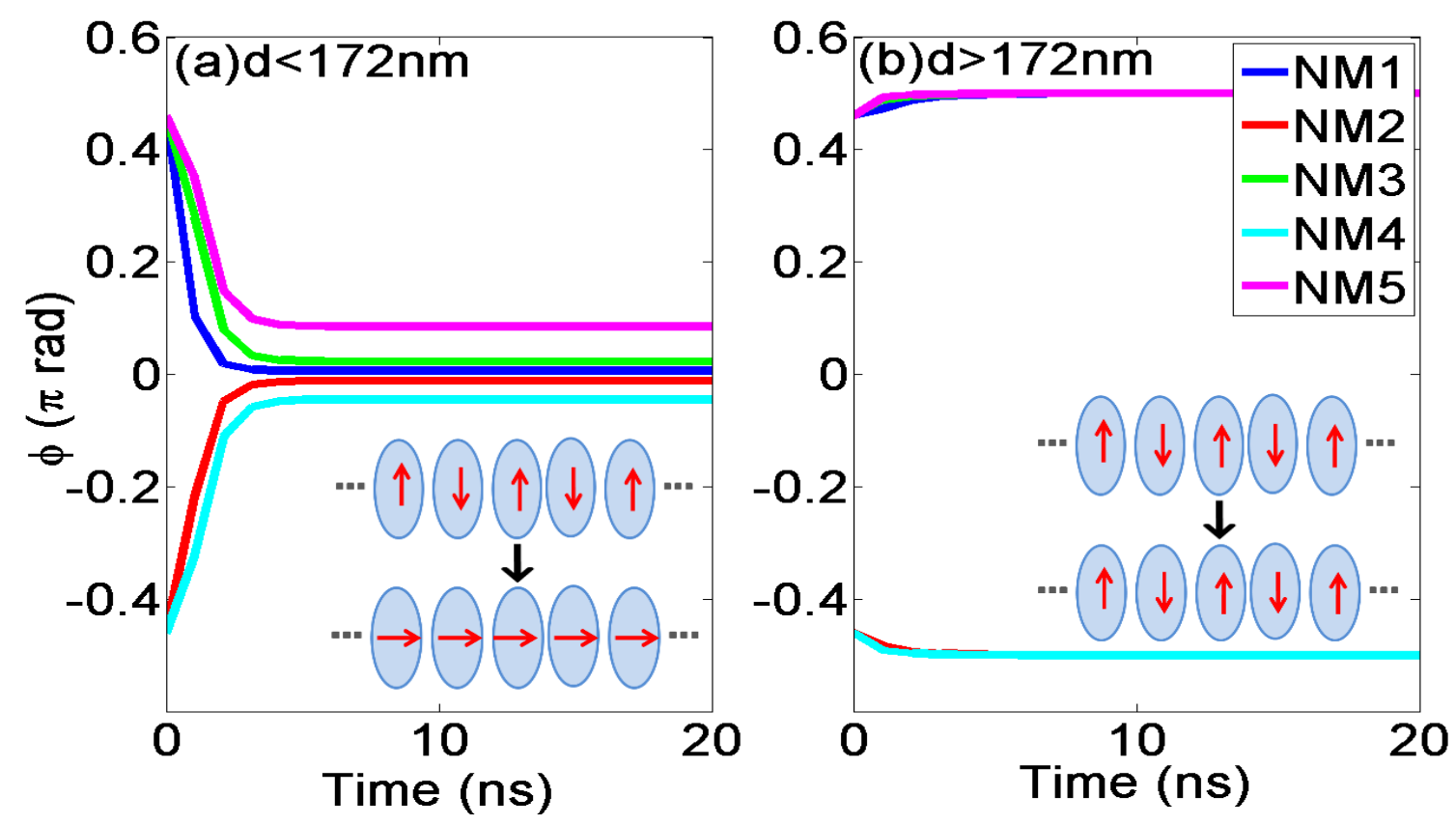

Figure 6.3: (a) Below a critical NM spacing, $d=172 \mathrm{~nm}$, the magnetizations of the NMs in a semi-finite array are energetically favorable along the minor axes, losing their alternating bits ' 0 ' $(\phi=\pi / 2)$ and ' 1 ' $(\phi=3 \pi / 2)$ informations. (b) The spacing between the NMs need to be more than $172 \mathrm{~nm}$ for the information stored in the Terfenol-D layer to be intact.The results in this figure is for 0K temperature.

In this study, we have assumed that the magnetostrictive $(3 / 2) \lambda_{S}=9 \times 10^{4}$ and $M_{S}=0.8 \times 10^{6} A m^{-1}$ [85]. The Gilbert damping constant for Terfenol-D is $\alpha=0.1[86]$. The demagnetization energies of the NMs are sufficiently high $\left(\approx 32 K_{B} T\right.$ at room temperature) so that the equilibrium bit error probability due to spontaneous magnetization flipping is very low $\left(e^{-32}\right)$.

The geometry of the NM array must be designed to utilize the dipolar couplings suitably. As $a, b \gg t$, the demagnetization factors, $N d_{y y}$ and $N d_{x x}$ are $\ll N d_{z z}$. This means that the magnetization prefers to lie in the $\mathrm{x}-\mathrm{y}$ plane $(\theta \approx \pi / 2)$, where $\mathrm{y}$ is the major(easy) axis and $\mathrm{z}$ is the $\operatorname{minor}($ hard $)$ one. At $\theta \approx \pi / 2$, $E_{\text {dipole }}=\mu_{0} M_{S}^{2} \Omega_{i} \Omega_{j} /\left(4 \pi R^{3}\right)\left[-2 \cos \phi_{i} \cos \phi_{j}+\sin \phi_{i} \sin \phi_{j}\right] \approx \beta\left[-2 \cos \phi_{i} \cos \phi_{j}+\sin \phi_{i} \sin \phi_{j}\right]$. If the two NMs are anti-ferromagnetically coupled with their magnetization along the major axes, $E_{\text {dipole } \downarrow}=-\beta$, while if the are in parallel configuration, $E_{\text {dipole } \uparrow}=\beta$. However, if the magnetizations of both the NMs are along the minor axes, $E_{\text {dipole } \rightarrow \rightarrow} \rightarrow$ is at the lowest at $-2 \beta$. Therefore, when the NMs are in anti-parallel configuration in ground state, the demagnetization field is strong enough to keep the magnetizations aligned along the major axes. However, if the NMs are too close to each other, the dipole-dipole interaction is stronger than the demagnetization field and then magnetizations of the NMs tend to align to the minor axes, thereby losing their bit ' 0 ' or ' 1 ' information.

Fig. 6.3 shows that when the NMs are spaced less than $172 \mathrm{~nm}$ apart, they lose their information. The 

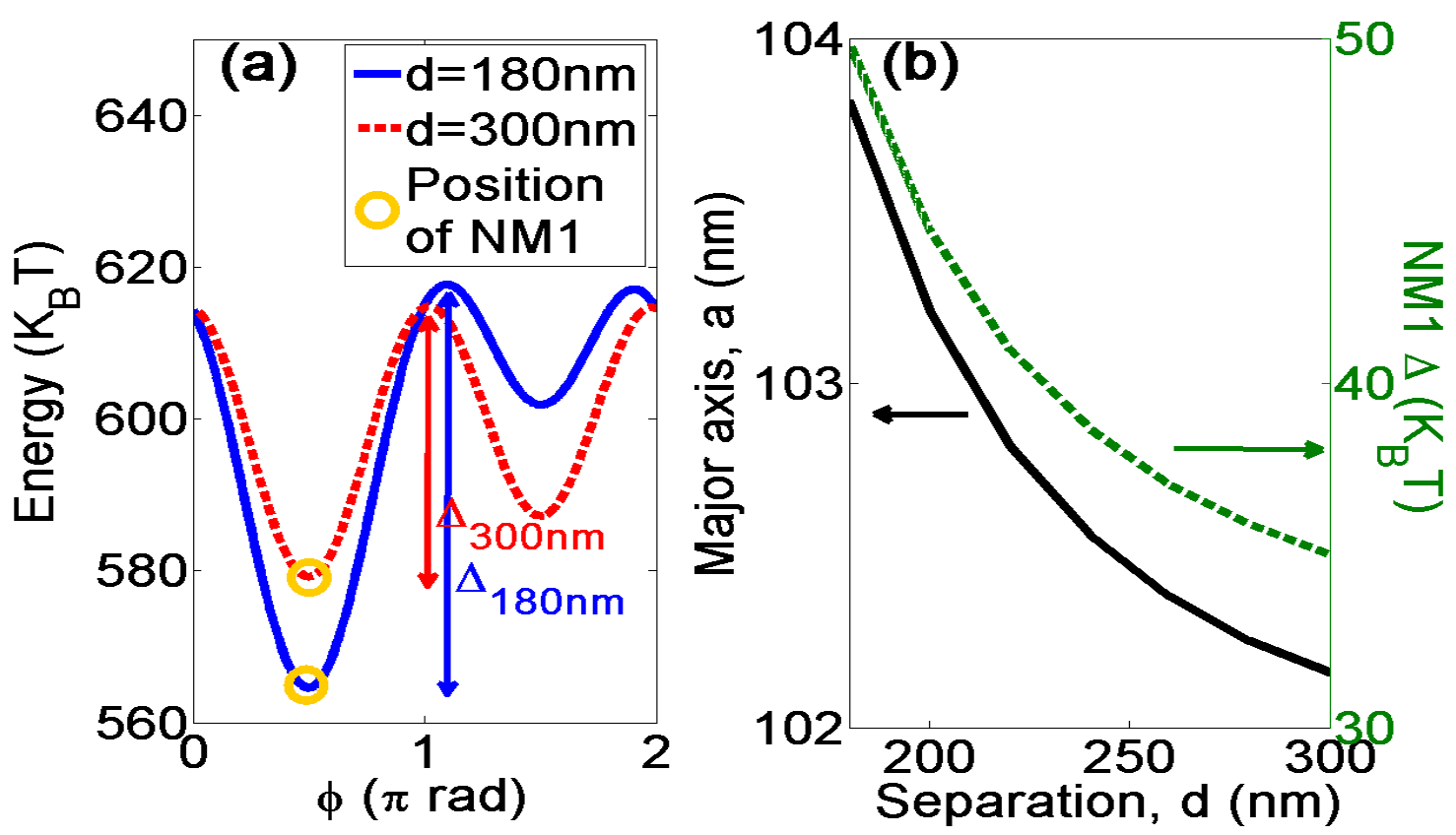

Figure 6.4: (a) Energy profile of NM1 with the dipolar coupling with its neighbors to the left taken into account. As the spacing between the NMs, d, is increased from $180 \mathrm{~nm}$ to $300 \mathrm{~nm}$, the energy barrier between the two stable configuration of NM1 decreases from $49.85 K_{B} T$ to $35.02 K_{B} T$. This is because the strength of the dipole coupling is proportional to $1 / d^{3}$. (b) The energy barrier of NM1 decreases with increasing spacing with its neighbors. The major axis of NM1 is adjusted accordingly in our simplified 2 NMs system is increased to account for the dipolar coupling to its left neighbors.

NMs need to more than $172 \mathrm{~nm}$ apart to retain their bit information. For the study done in this paper therefore, we will assume a 200nm spacing between the NMs, allowing for a $\pm 10 \%$ manufacturing variance[77], with the lower end of the variance still above the critical NM separation.

Instead of simulating the entire binary wire, we are interested in the information transfer between two NMs along a hypothetical array. To capture the influence of the missing left neighbors of our simplified two NM system (Fig 6.1b), the major axis of NM1 is increased to account for the dipolar coupling to its left neighbors (Fig. 6.4). In order words, the energy barrier due to demagnetization field between the major and minor axes is increased to take dipole-dipole interaction with the left neighbors into account. The NMs to the right of NM2 are disregarded in this study (they will be Bennett clocked into erasure during this step), and the size of NM2 is thus kept intact. In a binary wire, if the left neighbor of NM1 has spin down magnetization, NM1 will be at minimum energy at $\phi=\pi / 2$ (magnetization up). As the spacing ' $\mathrm{d}$ ' between NMs is increased, the energy barrier between the two stable configurations of NM1 decreases, because the dipole coupling is proportional to $1 / d^{3}$ (Fig. 6.4a). Accordingly, in our 2 NMS simplified system, the major axis of NM1 is adjusted with the separation d (Fig. 6.4b). For a separation of 200nm, the major axis of NM1 is taken to be $103.25 \mathrm{~nm}$ with a corresponding barrier of $42 K_{B} T$. The additional barrier accounts for the 


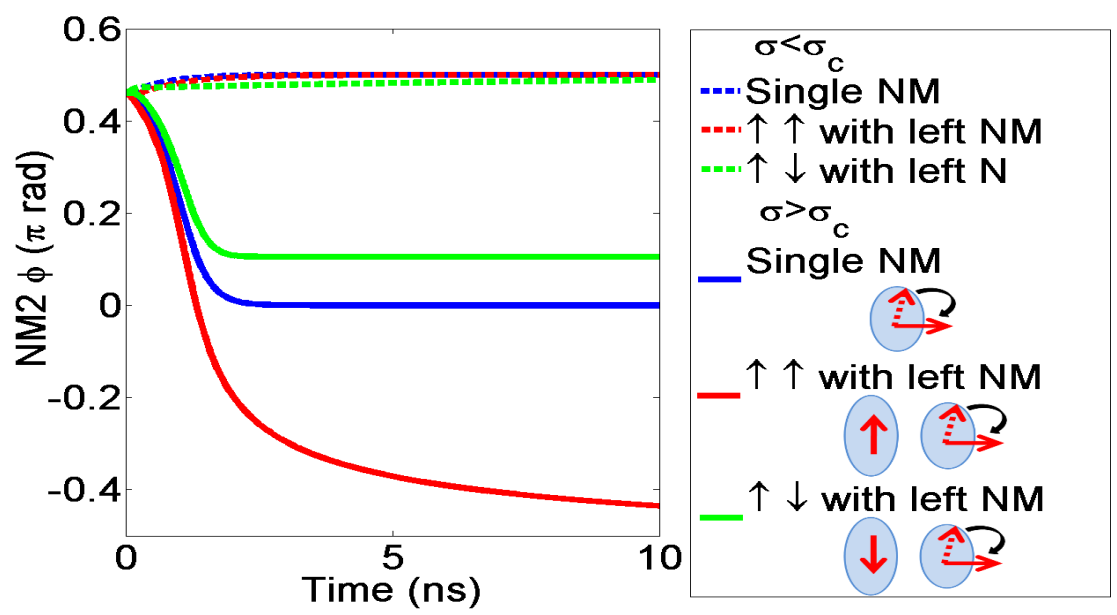

Figure 6.5: The critical stresses required to Bennett-clock NM for a single NM, in $\uparrow \uparrow$ configuration with its left neighbor and in in $\uparrow \downarrow$ configuration with its left neighbor are $1.84 \mathrm{MPa}, 1.14 \mathrm{MPa}$ and $2.53 \mathrm{MPa}$ respectively at $200 \mathrm{~nm}$ separation. If stress applied is less than that of the critical stress (1.8MPa for single NM, $1 \mathrm{MPa}$ for $\uparrow \uparrow$ configuration with its left neighbor, $2.3 \mathrm{MPa}$ for $\uparrow \downarrow$ configuration with its left neighbor), the magnetization of the stressed NM do not switch. If stress applied is greater than that of the critical stress (2.5MPa for single $\mathrm{NM}, 2 \mathrm{MPa}$ for $\uparrow \uparrow$ configuration with its left neighbor, $2.8 \mathrm{MPa}$ for $\uparrow \downarrow$ configuration with its left neighbor), the magnetization of the stressed NM switch close to its hard axis. The results in this figure is for 0K temperature.

dipolar coupling to its left neighbors.

The critical stresses required to Bennett-clock NM2 in the $\uparrow \uparrow$ configuration vs the $\uparrow \downarrow$ configuration with NM1 are:

$$
\begin{gathered}
\sigma_{\text {single } N M}^{C}=\frac{\mu_{0}}{2 \lambda} M_{S}^{2}\left[N d_{z z}-N d_{y y}\right] \\
\sigma_{\uparrow \uparrow \text { with } N M 1}^{C}=\frac{\mu_{0}}{2 \lambda} M_{S}^{2}\left[N d_{z z}-N d_{y y}-\frac{\Omega_{N M 1}}{2 \pi d^{3}}\right] \\
\sigma_{\uparrow \downarrow \text { with } N M 1}^{C}=\frac{\mu_{0}}{2 \lambda} M_{S}^{2}\left[N d_{z z}-N d_{y y}+\frac{\Omega_{N M 1}}{2 \pi d^{3}}\right]
\end{gathered}
$$

The numbers are $1.84 \mathrm{MPa}, 1.14 \mathrm{MPa}$ and $2.53 \mathrm{MPa}$ respectively at $200 \mathrm{~nm}$ separation. If stress applied is less than that of the critical stress, the magnetization of the stressed NM2 does not switch (Fig. 6.5). If on the other hand, the stress applied is greater than the critical stress, the magnetization of the stressed NM2 switches to its hard axis. For a stress of $2 \mathrm{MPa}$ for $\uparrow \uparrow$ configuration with its left neighbor, the magnetization switches to $-\pi / 2$ instead of 0 . This phenomena is interesting and can be understood better by studying the energy landscape of the NM2 with a small applied stress at 0 Kelvin.

Fig. 6.6a shows the energy landscape and minimum energy position of the magnetization of NM2, spaced 

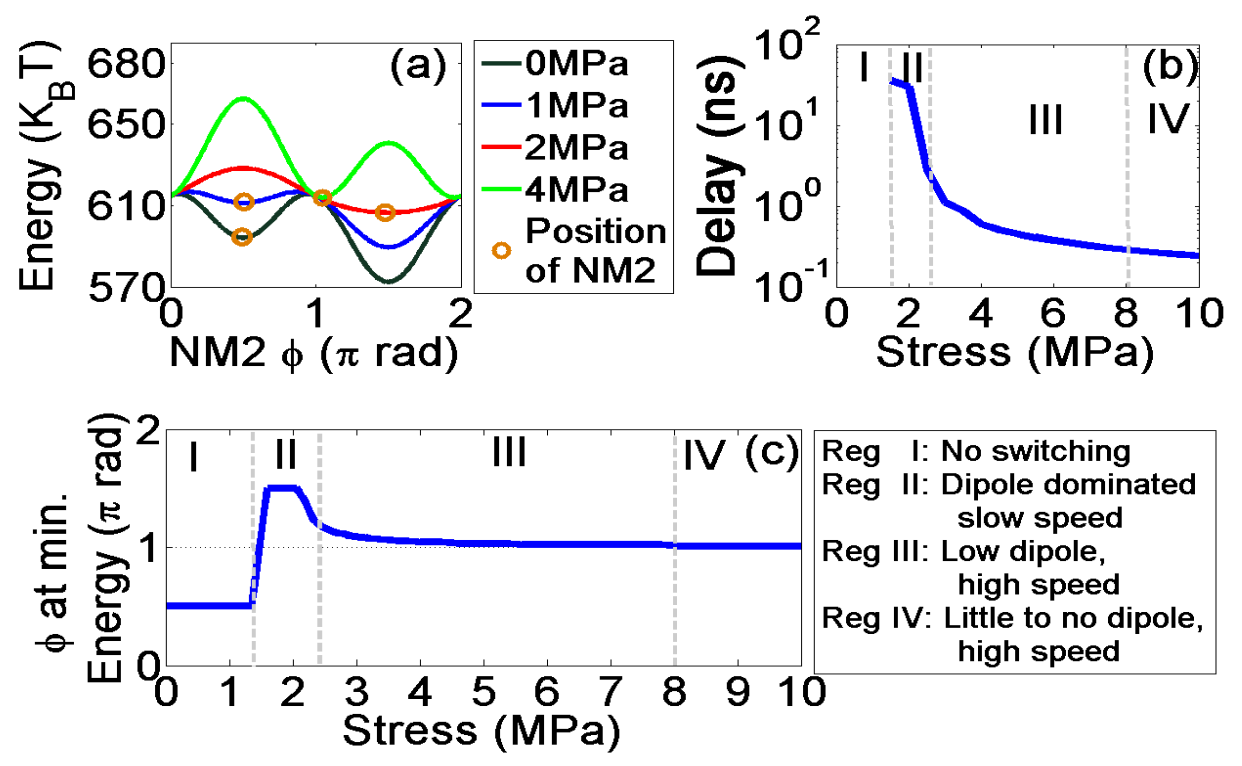

Figure 6.6: (a) Energy landscape and and changing position of the magnetization of NM2 with varied stress. At stress level below that of critical, the magnetization of NM2 is at the local minimum and initial position, $\phi=\pi / 2$. At $2 \mathrm{MPa}$, the stress applied cancels out the demagnetization field, and the dipole interaction with NM1 dominates the energy landscape. The energy minimum for $2 \mathrm{MPa}$ is near the intended destination, $\pi=\pi / 2$. However, $2 \mathrm{MPa}$ applied stress takes a long time to switch (60ns) as shown in (b) At higher stress, energy minimum shifts to the hard axis, $\phi=\pi$ and its takes a shorter time to switch. (c) $\phi$ at energy minimum for varying stresses. At Region I, for stresses below that of critical, energy minimum is at $\pi / 2$ and the magnetization doesn't switch at all. From 1.85 to $2.5 \mathrm{MPa}$, region II or dipole dominated region, stress cancels out the demagnetization field and only the dipolar effect dominates the energy landscape. The minimum energy $\phi$ is close to $3 \pi / 2$ very close to the intended destination. However it takes a long time for the magnetization to switch. The high speed regions III and IV, stress starts to dominate the energy landscape and there is low and little to no dipole effect respectively. The magnetization switches pretty fast in Region III and IV.

200nm from NM1. At a stress level below critical, the magnetization of NM2 is at the local minimum and initial position, $\phi=\pi / 2$. Close to the critical stress level at $2 \mathrm{MPa}$, the applied stress cancels out the demagnetization field, and the dipole interaction with NM1 dominates the energy landscape (we refer to it as the dipole-dominated regime). The energy minimum for $2 \mathrm{MPa}$ is near the intended destination, $\phi=3 \pi / 2$. However, switching with 2MPa stress takes a long time, around 60ns (Fig 6.6b). At higher stress, the energy minimum shifts to the hard axis, $\phi=\pi$ and it takes a shorter time to Bennett-clock. Fig. 6.6c shows $\phi$ at its energy minimum for varying stresses. In Region I for sub-critical stresses the energy minimum is at $\pi / 2$ and the magnetization does not switch at all. For the dipole dominated regime between 1.85 to $2.5 \mathrm{MPa}$, the stress cancels out the demagnetization field and only the dipolar effect dominates the energy landscape. The minimum energy $\phi$ is close to $3 \pi / 2$, very close to the intended destination. However it takes a long time for the magnetization to switch. In Regions III and IV, the stress starts to dominate the energy landscape and there is little to no dipole effect. The magnetization switches pretty fast in Regions III and IV, which are the 

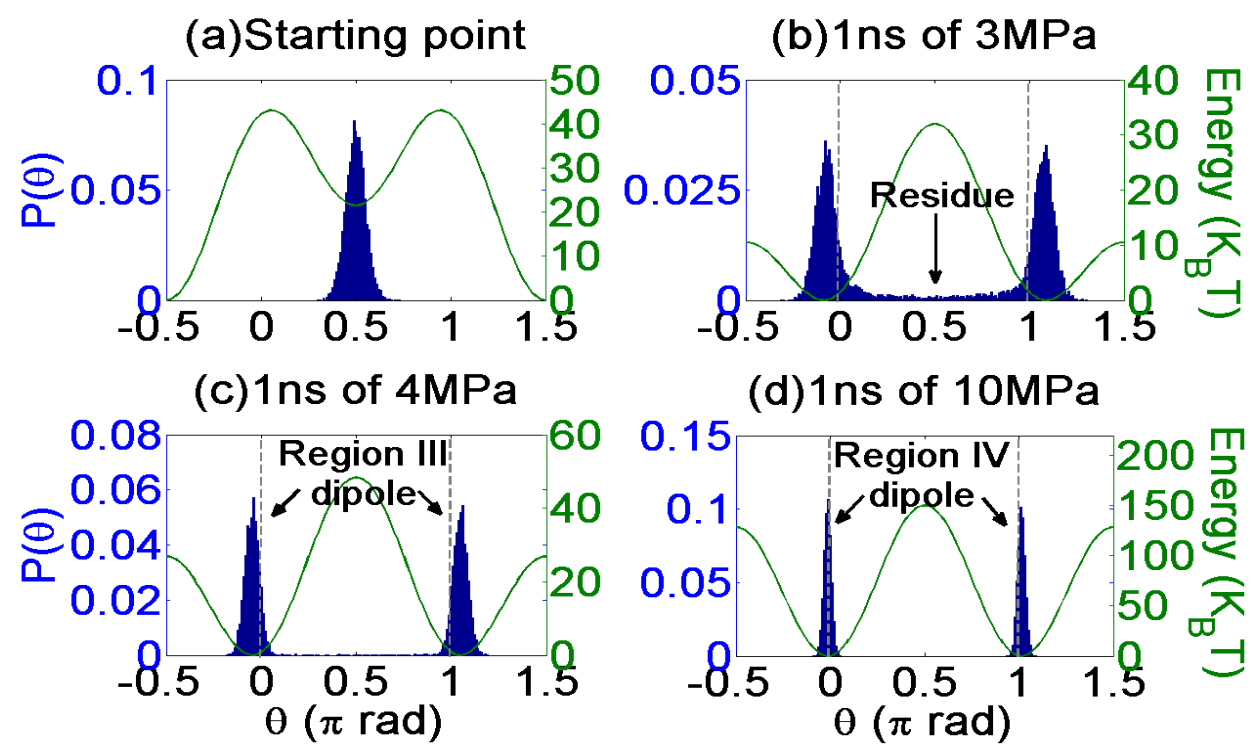

Figure 6.7: (a) Distributions of the polar azimuthal angle $\phi$ of NM2 due to thermal fluctuations during Period A at room temperature. The mean of the distribution is at stagnation point, $\phi=\pi / 2$. (b) An applied stress of $3 \mathrm{MPa}$ for $1 \mathrm{~ns}$ is not enough to kick all the magnetization out of the stagnation point and toward the hard axis. (c) An applied stress of $4 \mathrm{MPa}$ for $1 \mathrm{~ns}$ is strong enough to kick most of the magnetization out of the stagnation point. Around the hard axes, the effect of dipole coupling with NM1 is stronger than the stress, the system therefore operating in Region III dipole effecr(Fig. 6.6c). The peak of the switched distribution is away from the hard axes, $\phi=0$ or $\pi$. (d) The peak of the distribution is along the hard axes, the system operating in Region IV dipole effect after 1ns applied stress of 10MPa.

high speed regions, but since the dipole plays a minimal role, i.e., the distribution is pinned much tighter to the hard axis with little asymmetry, there is a larger probability of backflow to the origin rather than the destination, increasing thereby the error rate. These examples how the interplay between the demagnetization field, applied stress and dipolar interactions between the NMs is crucial in determining the dynamic error rate during information transmission between two NMs.

\subsection{Information transmission between two NMs taking thermal perturbations into account}

Fig. 6.7a shows the distribution of the azimuthal angle $\phi$ of NM2 set in a parallel configuration with NM1, spaced $200 \mathrm{~nm}$ apart. The thermal perturbations are included by a Langevin random field $\vec{H}_{L}$ that can be added to the effective magnetic field term. The field $\vec{H}_{L}$ relates to the system temperature $\mathrm{T}$ by,

$$
\vec{H}_{L}=\sqrt{\frac{2 \alpha K_{B} T}{\mu_{0} \gamma \Omega M_{S} \delta t}} \vec{G}
$$


where $\vec{G}$ is a Gaussian white noise along three uncorrelated dimensions, with zero mean and unit standard deviation [67]. $\delta t$ is the simulation time-step used to solve the coupled Landau-Lifshitz-Gilbert equation numerically. The mean of the distribution is at the stagnation point, $\phi=\pi / 2$, where $\delta \phi / \delta t=0$ for an applied stress. Thus the initial angles close to $\phi=\pi / 2$ have to wait till either thermal perturbations or a high level of stress dislodges the magnetization away from the stagnation point, thereby initiating switching towards the hard axis.

The success rate for the switching of NM2 depends therefore on two factors: (i) a high enough stress to kick the magnetization from its stagnation points $(\phi=\pi / 2$ or $3 \pi / 2)$ to the hard axis $(\phi=0$ or $\pi)$ in Period $\mathrm{B}$, and (ii) a prominent dipolar coupling in Periods $\mathrm{B}$ and $\mathrm{C}$ to dislodge the magnetization from the hard axis to the antiferromagnetic configuration. In the following subsections, we present the performance (reliability, energy consumed and average switching delay) of three types of stressing profiles: (A) abrupt stress removal at the end of Period B, (B) tapered stress removal and (C) high stress of 1ns in Period B, followed by 1ns 2MPa of stress in Period C.

In addition to the energy dissipated in the clocking circuit (which will be discussed in the subsections), some additional energy is dissipated in the magnet itself when it reverses magnetization. This energy is calculated as:

$$
E_{d}=\int_{0}^{\tau} \frac{\alpha \gamma}{\left(1+\alpha^{2}\right) \mu_{0} M_{S} \Omega}\left|T_{E}(t)\right|^{2} d t
$$

where $T_{E}(t)$ is the effective torque acting on a nanomagnet due to the combined effects of shape anisotropy, stress and dipole interaction[23].

\subsubsection{Switching reliability with abrupt stress removal}

Case 1 embodies an abrupt removal of stress, using a voltage pulse of 1ns (Fig. 6.8). 3MPa of stress is not enough to kick all the stagnant spins by the end of Period B, leaving a residue near the starting point (Fig 6.7b). This residue results in a low success rate. At 4MPA (Fig. 6.7c, region III), the stress value reaches a balance where it is high enough to kick most of the magnetization out of stagnation point (near vanished residue). At the same time, the stress is low enough to allow the dipole to dictate the final destination. We see that in the asymmetry of the distributions about the hard axis. In this region, the maximum success rate is achieved. If now the stress is further reduced (region IV, Fig 6.7d), the dipolar influence gets eliminated and the distribution gets pinned symmetrically to the hard axis. This means that upon releasing the stress the spins have a large chance of diffusing back to the origin. We thus see a decrease in reliability.

Fig 6.8 shows the peaking of the reliability in region III. The highest success rate achieved with a 1 ns pulse is $96.85 \%$ with $4 \mathrm{MPa}$ stress, which is not high enough for reliable logic computation. The average delay 

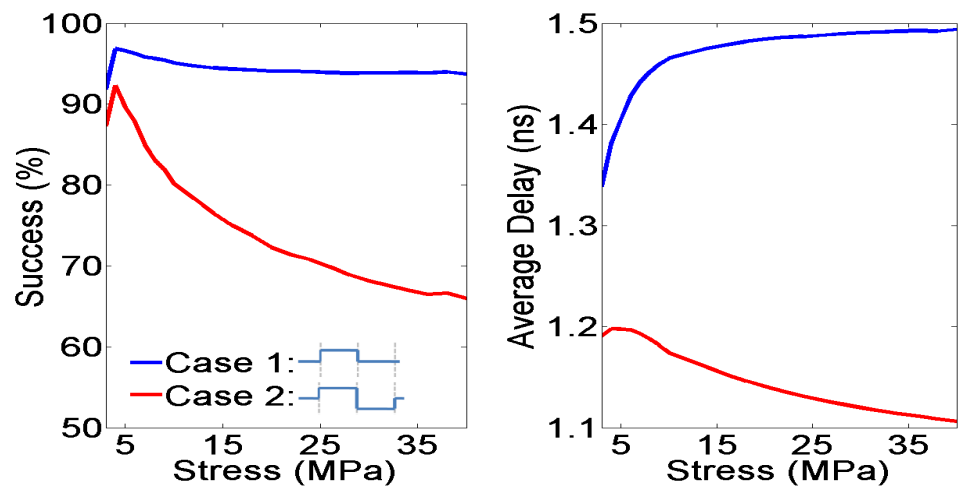

Figure 6.8: Success rate of Cases 1 and 2 stressing profile. For both the profiles, success rate is lower at small stress levels, peaks around $4 \mathrm{MPa}$ and then slowly deteriorates as stress is increased. Case 2 deteriorates mush faster than 1 . The average delay of case 1 is around $1.45 \mathrm{~ns}$, while the negative stressing profile of case 2 speeds up the switching to $1.15 \mathrm{~ns}$.
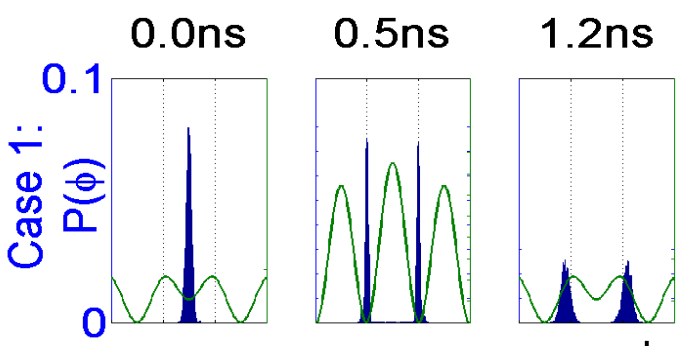

$1.5 \mathrm{~ns}$

3.0ns
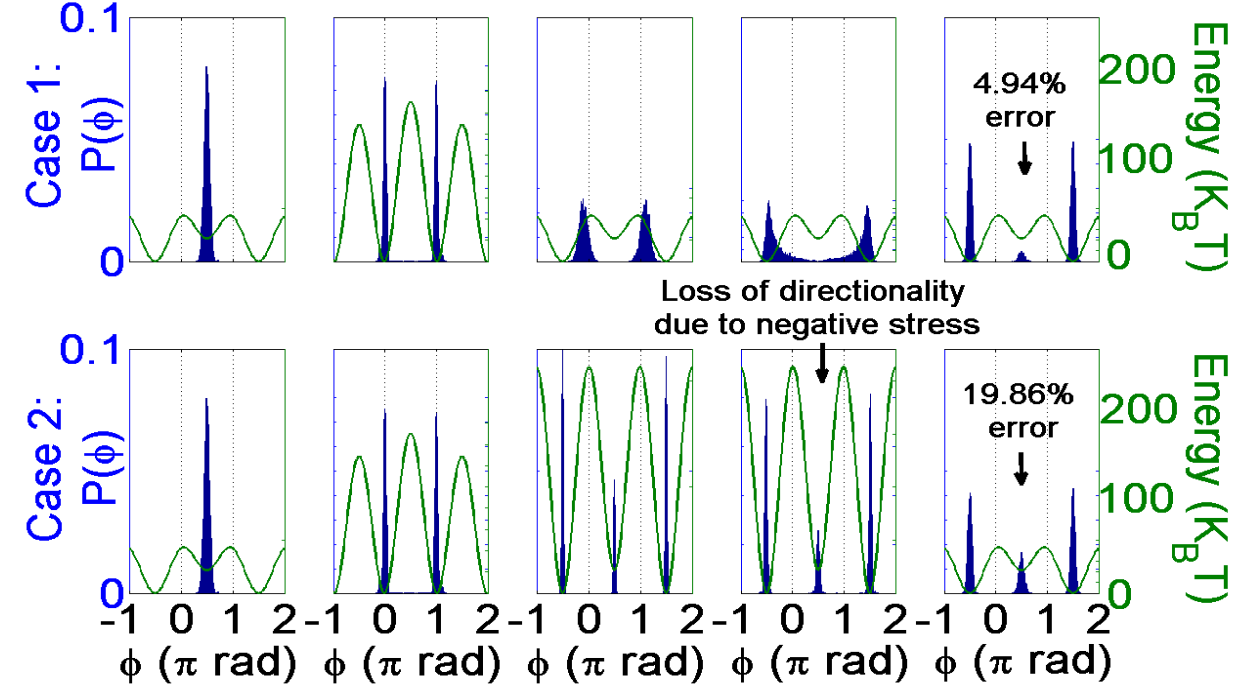

Loss of directionality due to negative stress

Figure 6.9: Comparison between cases 1 and 2 switching profiles at 10MPa. While the negative stress in case 2 speeds up the switching process, it erases some of the effect of dipolar coupling with NM1, results in a higher error rate.

is $1.45 \mathrm{~ns}$, while the energy dissipation is $C V^{2}$ where $\mathrm{C}$ is the capacitance of a $40 \mathrm{~nm}$ thick PZT layer (1.74 $\mathrm{fF}$ ) and $\mathrm{V}$ is the voltage applied. The voltage needed to generate a certain stress across the PZT layer is $V=\sigma t_{P Z T} /\left(Y \cdot d_{31}\right)$, where $t_{P Z T}$ is the thickness of the PZT layer, $\mathrm{Y}$ is the Young's modulus of Terfenol-D $\left(8 \times 10^{10} \mathrm{~Pa}[87]\right)$ and $d_{31}$ is the piezoelectric coefficient of PZT $\left(-10^{-10} \mathrm{mV}^{-1}[88]\right)$.

To switch faster, a negative stress is applied in Case 2, acting during Period C (Fig. 6.8). At higher stress, the reliability for Case 2 deteriorates much faster than 1 . This is because the negative stress during Period C overpowers the effect of dipolar coupling with NM1, resulting in increased back-hopping and a higher 

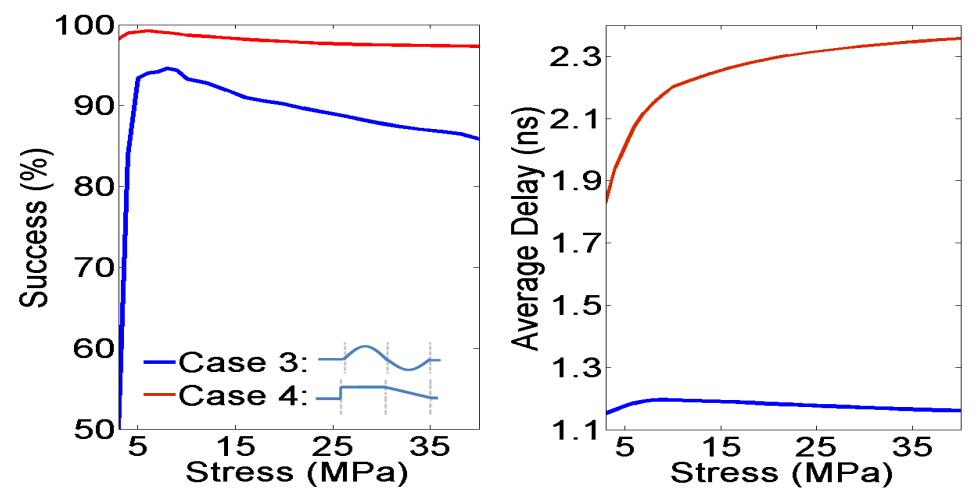

Figure 6.10: Success rate of Cases 3 and 4 stressing profile. While case 3 dissipates very little energy, the overall success rate is lower as the sinusoidal stress during Period B is not enough to kick all the magnetization out of the stagnation point and the negative stress during Period $\mathrm{C}$ overpowers the dipolar effect. Average switching delay is $1.2 \mathrm{~ns}$. Case 4 has better success rate with an average delay of $2.1 \mathrm{~ns}$.

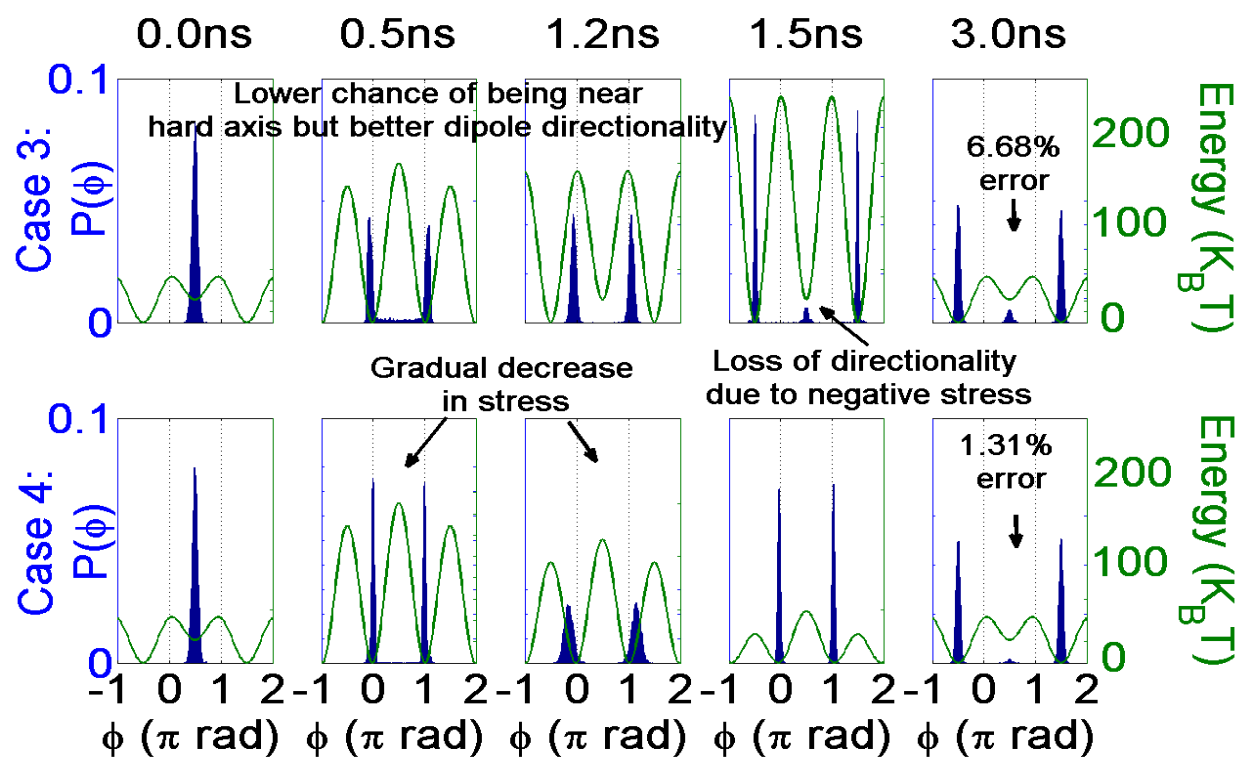

Figure 6.11: Comparison between cases 3 and 4 switching profiles at 10MPa. The overall error rate is higher in case 3 as the sinusoidal stress during Period B is not enough to kick all the magnetization out of the stagnation point and the negative stress during Period $\mathrm{C}$ overpowers the dipolar effect. Case 4 has lower error rate as the gradual decrease of stress allows the magnetization switching to operate in Region II for some time.

error rate (Fig. 6.9). The negative stressing profile of case 2 speeds up the switching to 1.15 ns, however the clocking energy dissipation is $3 C V^{2}[16]$.

\subsubsection{Switching reliability with tapered stress removal}

Case 3 is a very low-power, sinusoidal stress profile with a period of $2 \mathrm{~ns}$ (Fig. 6.10). Stress is removed slowly at the end of Period B and a negative stress during Period C switches the magnetization of NM2 faster. The 

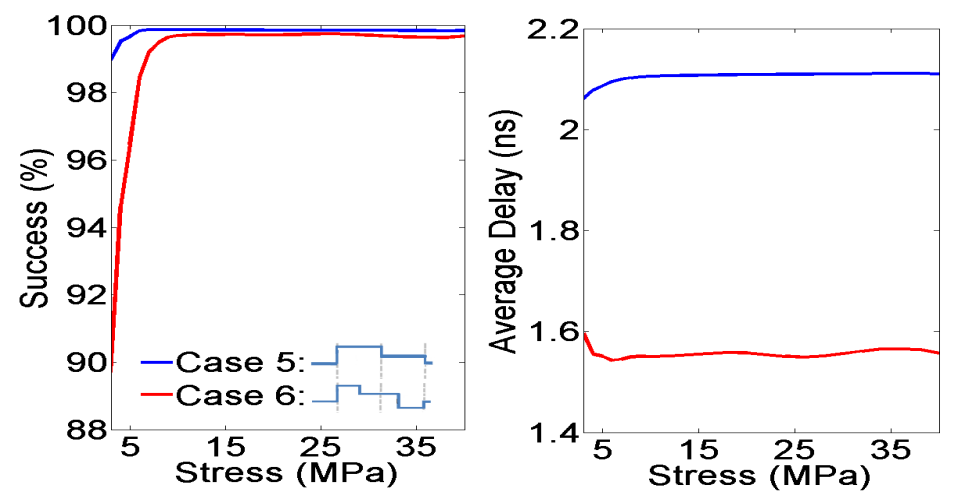

Figure 6.12: Comparison between cases 3 and 4 switching profiles at 10MPa. Operating at 1ns in dipole dominated region II dipole effect results in very low error rate. Average delay for case 5 is $2.1 \mathrm{~ns}$ while the negative stress is case 6 decreases the delay to $1.55 \mathrm{~ns}$.

tapered stress removal helps with reliability, as the stress operates in the dipole dominated region II for a brief period of time. However, the overall success rate is lower as the sinusoidal stress during Period B is not enough to kick all the magnetization out of the stagnation point and the negative stress during Period $\mathrm{C}$ overpowers the dipolar effect (Fig. 6.11). Average switching delay is 1.2ns. Clocking energy dissipation is $\eta C V^{2}$, where $\eta=\pi \omega R C / 3=5.47 \times 10^{-3}[16]$.

In Case 4, after keeping the stress on for 1ns, it is slowly removed in $1 \mathrm{~ns}$ instead of abruptly, achieving higher reliability than Cases 1,2, and 3. The slow removal of stress allows the magnetization switching to operate in the dipole dominated stress region for a short amount of time (Fig. 6.11). Energy dissipation is $C V^{2}$ while switching delay is $2.1 \mathrm{~ns}$. For both cases 3 and 4 , the success rate deteriorates as the stress level is increased, because we end up spending less time in Regions II and III.

\subsubsection{Switching efficiently using region II - the dipole dominated regime}

In section 6.2, we identified a range of stresses, referred to as Region II or the dipole dominated regime, where the applied stress cancels out the demagnetization field and only the dipolar effect dominates the energy landscape. The dominant dipole term leads to a better directionality to the switching magnetization after it has been brought to the hard axis by stress writing. In Case 5, the reliability is improved by cleverly stressing NM2 (Fig. 6.12). A high stress is applied to kick the magnetization out of the stagnation point, and then lowered it to 2MPA for 1ns (Fig. 6.13). 2MPa falls under Region II, where the dipole dominated energy landscape nudges the switching magnetization toward the final intended destination. The success rate is much higher $(99.87 \%$ for $10 \mathrm{MPa})$, with a delay of $2.1 \mathrm{~ns}$. The energy dissipated is $C V^{2}$.

In Case 6, a high stress is applied for $0.5 \mathrm{~ns}$, then $2 \mathrm{MPa}$ for $1 \mathrm{~ns}$ and finally a negative stress for $0.5 \mathrm{~ns}$ to push the magnetization towards its destination. For a slightly lower success, 99.71\%, and energy cost, the 


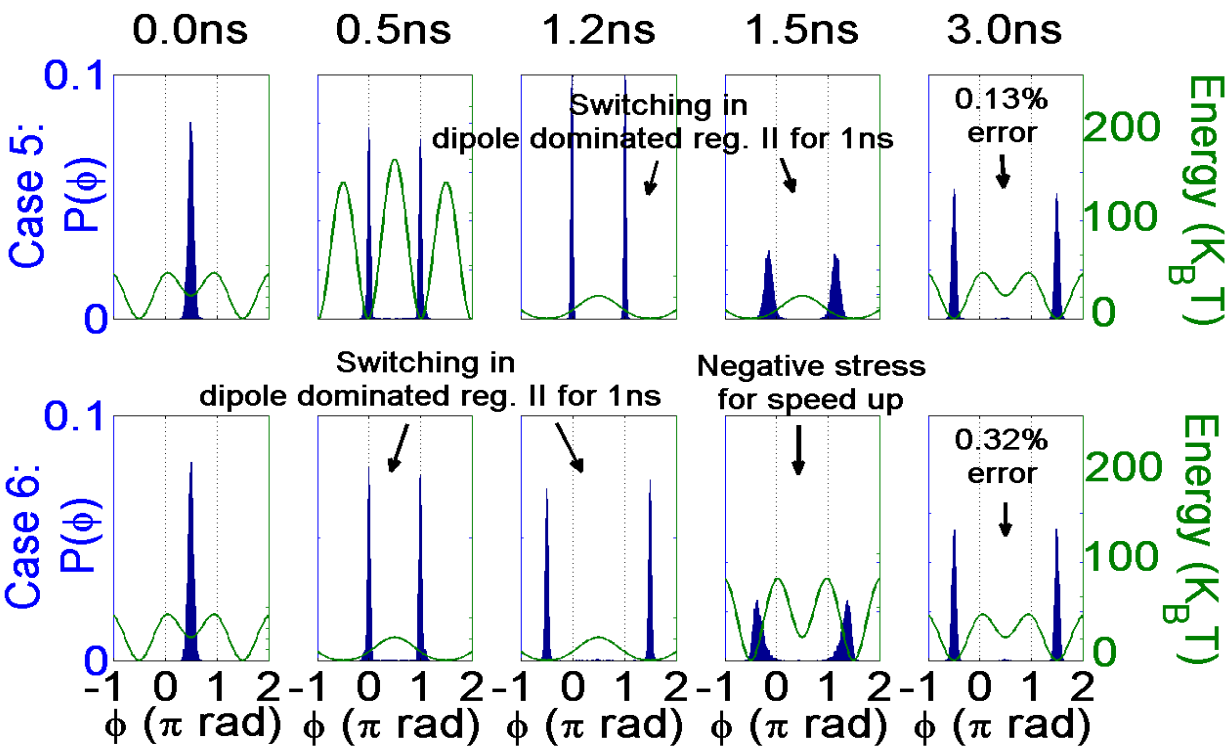

Figure 6.13: Operating at $1 \mathrm{~ns}$ in dipole dominated region II dipole effect results in very low error rate. Switching speed is increased by a negative stress in case 6 .

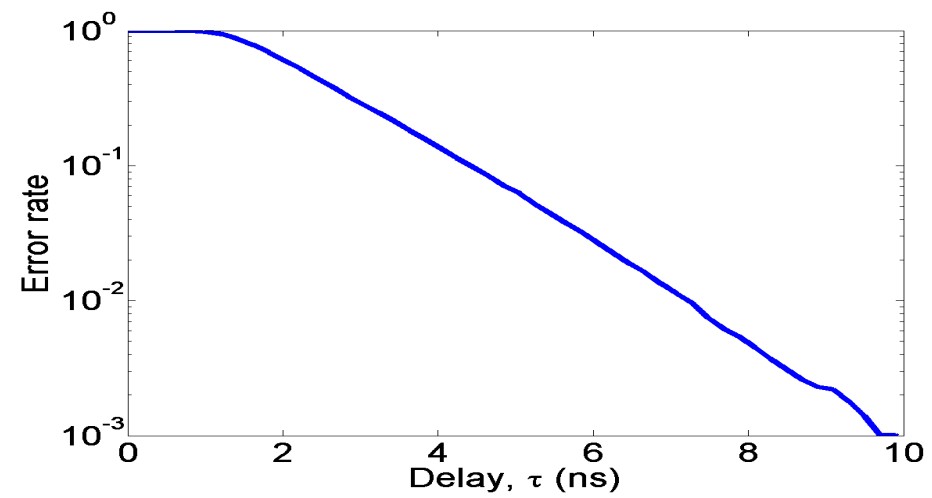

Figure 6.14: Very low error rate can be achieved by continuing to stress NM2 at 2MPa after 1ns stress of $1 \mathrm{MPa}$. Error rate of $10^{-3}$ is achieved at $10 \mathrm{~ns}$.

profile shaves off $0.6 \mathrm{~ns}$ of switching time. The energy dissipation is $C V^{2}+126 K_{B} T$ where the extra energy is due to the abrupt swing from $+2 \mathrm{MPa}$ to $-2 \mathrm{MPa}$.

One might argue that the best case scenario (case 5) with a success rate of $99.87 \%$ is not good enough for logic computation. If NM2 is continued to be stressed at 2MPa, a much lower error rate can be achieved (Fig. 6.14). However, this low error rate comes at the expense of longer switching delay, 10ns for error rate of $10^{-3}$.

\subsection{Performance comparison between various stressing profiles}

Though multiferroic logic is energy-efficient, it needs improvement to be used as a tool for reliable and fast computation. The slow speeds and lower reliabilities are in fact, typical in nanomagnetic logic. In this work, 
Table 6.1: Energy dissipation, error rate and delay comparison of various stressing profile in nanomagnetic logic using multiferroics

\begin{tabular}{|c|c|c|c|c|}
\hline Case & $\begin{array}{c}\text { Peak success } \\
\text { rate at }(\mathrm{MPa})\end{array}$ & $\begin{array}{c}\text { Success rate } \\
(\%)\end{array}$ & $\begin{array}{c}\text { Energy dissipation } \\
\left(K_{B} T\right)\end{array}$ & $\begin{array}{c}\text { Delay } \\
(\mathrm{ns})\end{array}$ \\
\hline 1 & 4 & 96.85 & $E_{d}+C V^{2}$ & 1.38 \\
\hline 2 & 4 & 92.32 & $E_{d}+3 C V^{2}$ & 1.20 \\
\hline 3 & 8 & 94.56 & $E_{d}+\eta C V^{2}$ & 1.20 \\
\hline 4 & 6 & 99.22 & $E_{d}+C V^{2}$ & 2.07 \\
\hline 5 & 7 & 99.87 & $E_{d}+C V^{2}$ & 2.10 \\
\hline 6 & 12 & 99.72 & $E_{d}+C V^{2}+126 K_{B} T$ & 1.54 \\
\hline
\end{tabular}

we compared various stressing profiles in terms of information transmission reliability, energy dissipation and switching delay (Table 1). In order to ensure reliable information transmission between 2 NMs, a high stress is needed to kick the magnetization of the NM to the hard axes to Bennett-clock and then control the interplay between stress and the dipole interaction with the neighboring NM to switch to the intended destination. Just applying a 1ns stress pulse is not enough, as it results in $96.85 \%$ success rate for NMs spaced 200nm apart, dissipating energy in the order of $C V^{2}$. A sinusoidal stress pulse results in very low power computation at an energy $\eta C V^{2}$ where $\eta \approx 10^{-3}$. However, reliability is very poor, the best performance being 94.56\%. Applying a negative stress in the end speeds up the switching process, as in the cases 2 and 3 (1.2ns) compared to case 1 (1.38ns). The increased switching speed comes at the expense of lower success rate.

The reliability can be improved by smartly stressing NM2. A high speed stress can be applied to kick the magnetization out of stagnation point, and then lowered to the dipole dominated 2MPa level for $1 \mathrm{~ns}$ (case 5), achieving an optimization in the energy-delay-reliability trade-off. Success rate is high $99.87 \%$, with a delay of 2.1ns. In Case 6, a negative stress after the improvised stressing push the magnetization towards its destination. For a slightly lower success and higher energy cost, 0.6ns of switching time is shaved off. 


\section{Chapter 7}

\section{Conclusion and future work}

The aim of this dissertation is to meet the challenges posed in magnetic memory and logic: low reliability, high energy computation and computational delay. A unified model is presented that enables us to delve into the working of a STT-RAM bit cell at different level of complexities and suggest performance improvements. Material recommendations for single-barrier STT-RAM are made. A similar unified model is set up for straintronics based multiferroic logic, where we find that the error rate and switching delay can be controlled by material design and by engineering the stress profile on the nanomagnets. In the following sections, some ideas are listed that can further enhance the performance of STT-RAM and nanomagnetic logic.

\subsection{Faster and more reliable writing using external field + spin- transfer torque}

Low reliable writing in spintronic devices limits their applicability in the automotive and defense industries. Fortunately, greater reliable switching can be achieved in Magnetic Random Access Memory (MRAM) at the cost of greater energy dissipation. Using a combination of spin-transfer torque and small applied perpendicular field in MRAM, the error rate can be considerably reduced for a given voltage pulse.

Higher thermal stability contributes to longer switching tails, as the magnetization tends to be nearer to the stagnation point, $\theta=0$ or $\pi$, in the absence of an external perturbation. Near the stagnation point, the torque proportional to $\sin \theta$ is too small to initiate switching. Therefore a thermally stable free layer would require a relatively high current to have very low error rate $10^{-9}$ for switching within 5-10 ns. For an elliptical disk of $\mathrm{CoFeB}$ (minor axis $=40 \mathrm{~nm}$, major axis $=80 \mathrm{~nm}$ and thickness $=3 \mathrm{~nm}, M_{S}=1050 \mathrm{emu} / \mathrm{cc}$, $\left.H_{K}=500 \mathrm{Oe}, \alpha=0.02\right)$, an applied current of 10 times the critical switching current ensures switching with 
(a)

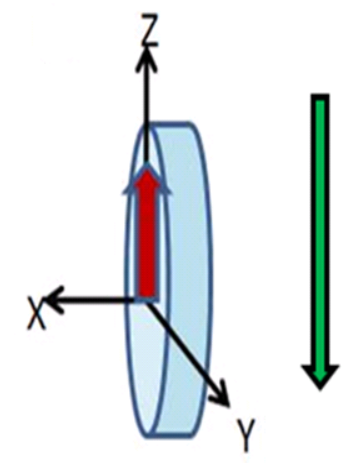

(b)

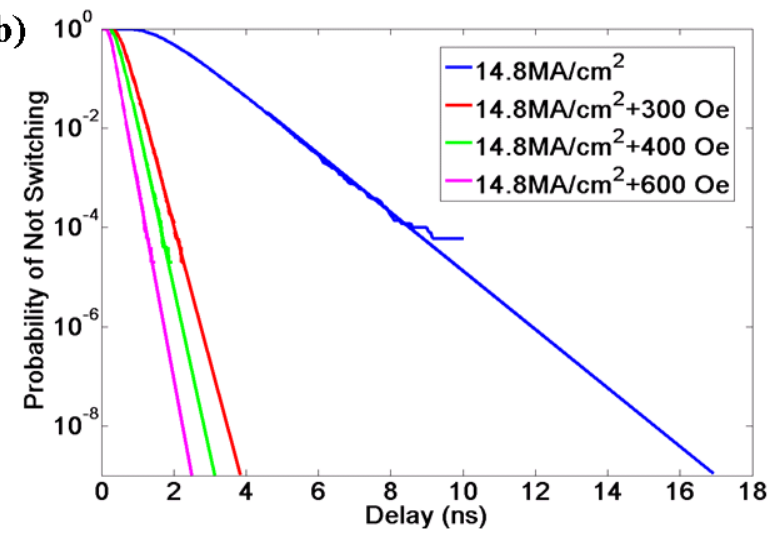

Figure 7.1: (a) Elliptical free layer where the magnetization prefers to lie along the major $(\mathrm{Z})$ axes. The external field is applied parallel along the direction of the spin torque during writing. (b) For a given error rate, an applied field in the direction of the spin torque considerably cuts down the delay. For a field greater than 3000 e, switching delay of less than $5 \mathrm{~ns}$ is needed for an error threshold of $10^{-9}$.

an error rate of $10^{-9}$ within a delay of $17 \mathrm{~ns}$ (Fig. 7.1b). An applied field in conjunction with spin-transfer torque switching reduces the delay considerably. For a field greater than 3000e, switching delay of less than $5 \mathrm{~ns}$ is needed for an error threshold of $10^{-9}$ Even though the applied external field reduces the delay, the energy consumption increases considerably (Table 7.1).

(a)

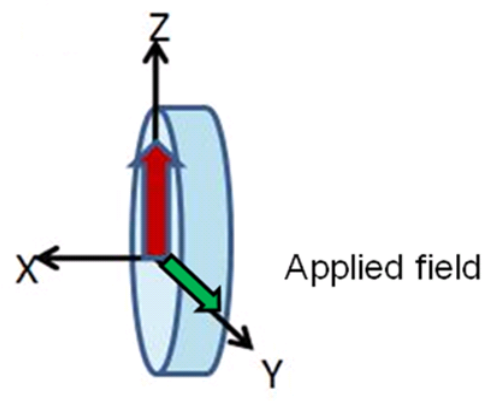

(b)

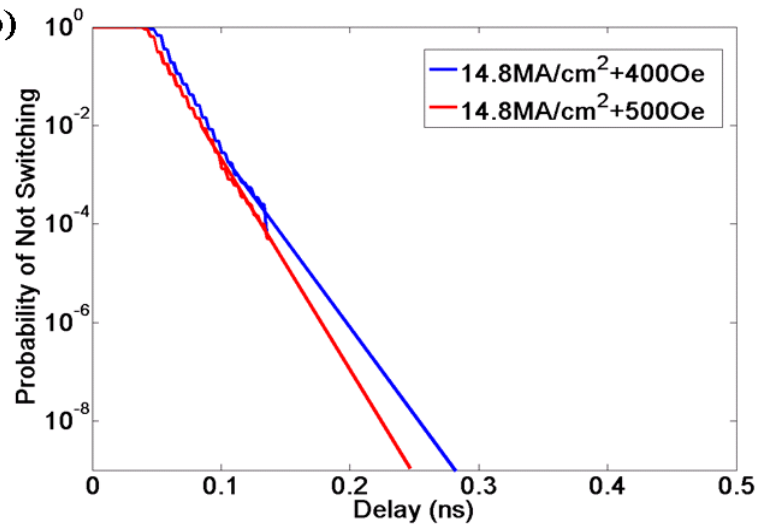

Figure 7.2: (a) Elliptical free layer where the magnetization prefers to lie along the major $(\mathrm{Z})$ axes. The external field is applied perpendicular to the magnetization for $0.1 \mathrm{~ns}$ at the beginning of the writing. (b) For a given error rate, an applied field in the direction perpendicular to the spin torque further cuts down the switching delay compared to the case where the field was applied parallel to the spin torque. For a field greater than $500 \mathrm{Oe}$, switching delay of less than $0.5 \mathrm{~ns}$ is needed for an error threshold of $10^{-9}$.

If the external field is applied perpendicular to the magnetization for $0.1 \mathrm{~ns}$ at the beginning of the writing (Fig 7.2a), the switching delay is further cut down, compared to the case where the field was applied parallel to the spin torque. For a field greater than 5000e, switching delay of less than 0.5ns is needed for an error threshold of $10^{-9}$ (Fig $7.2 \mathrm{~b}$ ). Torque is the highest when applied field is 90 degree to the starting point. The 
Table 7.1: Energy consumption during a write that ensures an error threshold of $10^{-9}$. A field is applied parallel to the spin torque current for the duration of the switching. Even though the applied external field reduces the delay, the energy consumption increases considerably

\begin{tabular}{|c|c|c|c|}
\hline Spin Torque current & Applied Field & Delay for $10^{-9}$ & Energy consumed \\
\hline $14.8 \mathrm{MA} / \mathrm{cm}^{2}$ & - & $17 \mathrm{~ns}$ & $1.897 \mathrm{pJ}$ \\
\hline $14.8 \mathrm{MA} / \mathrm{cm}^{2}$ & $300 \mathrm{Oe}$ & $4 \mathrm{~ns}$ & $117.45 \mathrm{pJ}$ \\
\hline $14.8 \mathrm{MA} / \mathrm{cm}^{2}$ & $400 \mathrm{Oe}$ & $3.2 \mathrm{~ns}$ & $166.75 \mathrm{pJ}$ \\
\hline $14.8 \mathrm{MA} / \mathrm{cm}^{2}$ & $600 \mathrm{Oe}$ & $2.4 \mathrm{~ns}$ & $292.78 \mathrm{pJ}$ \\
\hline
\end{tabular}

Table 7.2: Energy consumption during a write that ensures an error threshold of $10^{-9}$. A field is applied perpendicular to the spin torque current for $0.1 \mathrm{~ns}$. The perpendicular applied external field further reduces the delay, without consuming as much energy than that used during parallel applied field.

\begin{tabular}{|c|c|c|c|}
\hline Spin Torque current & Applied Field & Delay for $10^{-9}$ & Energy consumed \\
\hline $14.8 \mathrm{MA} / \mathrm{cm}^{2}$ & 400 Oe for $0.1 \mathrm{~ns}$ & $0.3 \mathrm{~ns}$ & $5.23 \mathrm{pJ}$ \\
\hline $14.8 \mathrm{MA} / \mathrm{cm}^{2}$ & 500 Oe for $0.1 \mathrm{~ns}$ & $0.2 \mathrm{~ns}$ & $8.15 \mathrm{pJ}$ \\
\hline
\end{tabular}

perpendicular field gets the magnetization out of the stagnation point fast. As the applied field is turned on for a very short time, energy consumption during write is much lower that the case when the field is parallel to the spin torque (Table 7.2). Further investigation is needed to study the energy-delay-reliability trade-off in applied+spin-transfer torque switching.

\subsection{Double barrier magnetic tunnel junction}

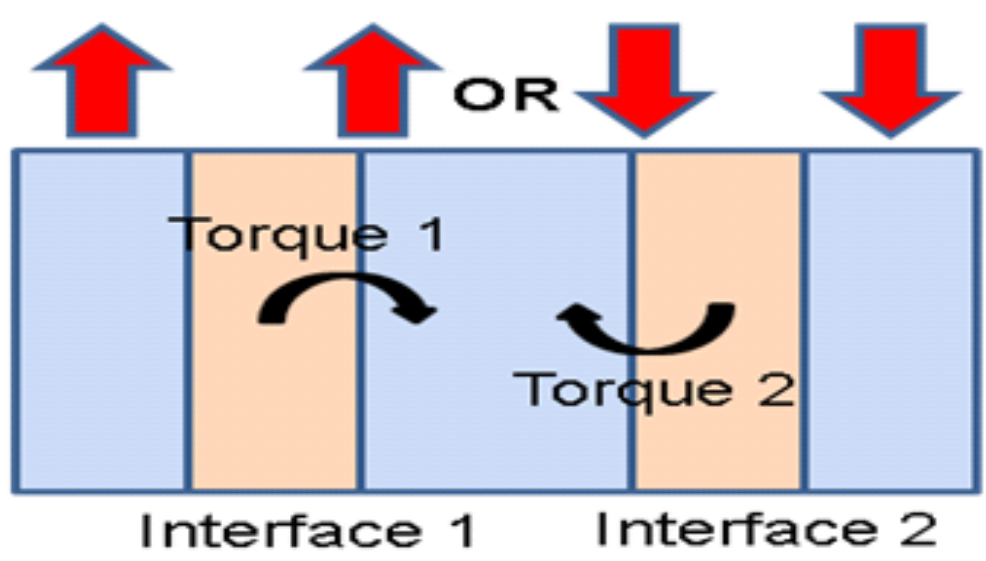

Figure 7.3: Fixed FM-Insulator-Free FM-Insulator-Fixed FM DMTJ system

As shown in the previous chapters, fast writing with low error threshold can be achieved in single barrier 
STT-RAM by driving a current greater than the critical current. However, generating such an overdrive current requires higher bias voltages $(>0.75 \mathrm{~V})$. Therefore, it is beneficial to boost the magnitude of spin torque to enable switching at lower current and voltage levels. The double magnetic tunnel junction (DMTJ) structure introduces another tunneling barrier and fixed layer on top of the free layer, as schematically depicted in Fig. 7.3. The magnetizations of the two fixed FM contacts are anti-parallel to each other. The advantage of this penta-layer system is that for an applied voltage, electrons from both the right and left fixed contact exerts a torque on the middle free layer $[89,55]$. If the middle layer is in majority up spin configuration, for an applied positive voltage, the left fixed layer will induce the $\mathrm{P}$ to AP switching mechanism while the right fixed layer will induce AP to $\mathrm{P}$ switching mechanism. Doubling the torque considerably reduces the voltage required to switch the free layer and time taken to switch, therefore saving on energy consumption.

However, when the insulators have the same width $(\mathrm{W} 1=\mathrm{W} 2)$, the DMTJ junction suffers from low TMR as the electrons have to tunnel through a high resistance anti-parallel path in both the configuration, $\uparrow \uparrow \downarrow$ and $\uparrow \downarrow \downarrow[90,91]$. One option is to make one of the insulating layers wider than the other, where the trilayer with the narrower insulator will dominate the TMR. The quasi-2D free layer FM film introduces Van-Hove singularities (Fig 7.4a) that can enhance the TMR[92, 93, 94, 95] and spin torque writing [96].
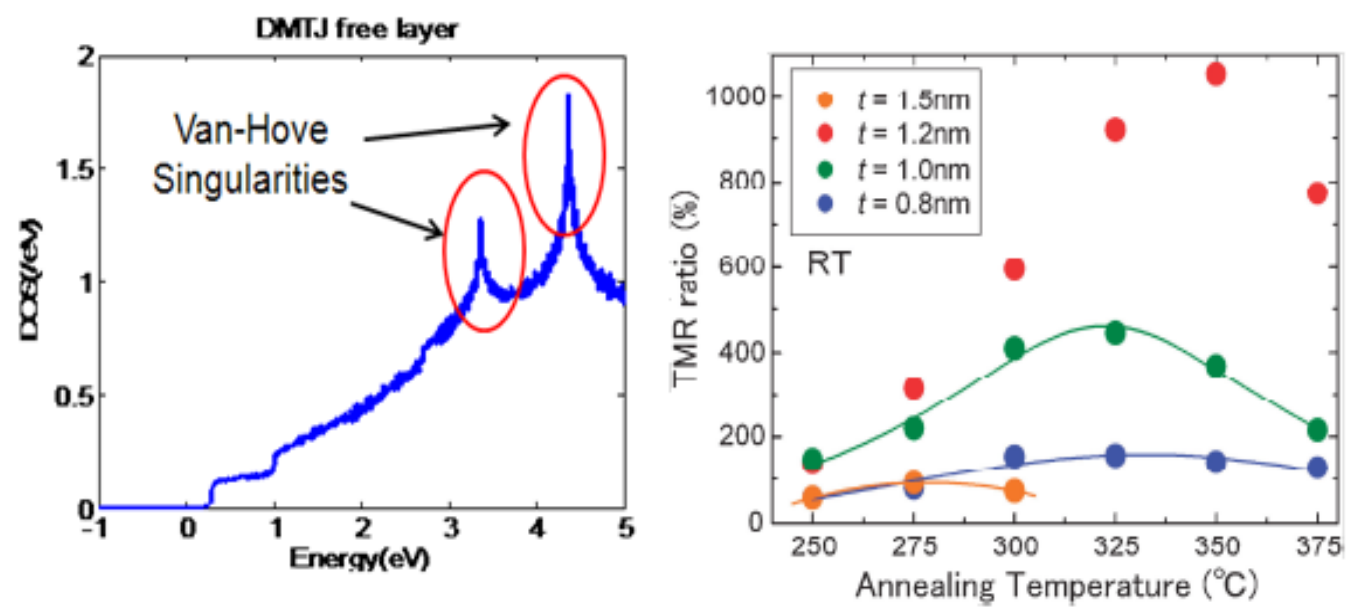

Figure 7.4: (a) Van-Hove singularities in the majority band of the free layer in a DMTJ. (b) TMR ratio as a function of annealing temperature for a $\mathrm{CoFeB} / \mathrm{MgO} / \mathrm{CoFeB}(\mathrm{t} \mathrm{nm}) / \mathrm{MgO} / \mathrm{CoFeB}$ DBMTJs with various middle layer thicknesses. A TMR of $1048 \%$ is reached when the free layer is $1.2 \mathrm{~nm}$

The Van-Hove singularities cause density of state to spike at given energy levels. A spike in the majority band will cause the polarization to spike as well, (pololarization $\left.=\left[D O S_{\uparrow}-D O S_{\downarrow}\right] /\left[D O S_{\uparrow}+D O S_{\downarrow}\right]\right)$, thereby resulting in increased TMR. Fig 7.4b and 7.5a and shows an experiment where the free layer of a $\mathrm{CoFeB} / \mathrm{MgO} / \mathrm{CoFoBe}(\mathrm{t} \mathrm{nm}) / \mathrm{MgO} / \mathrm{CoFeB}$ were varied from 0.8 to $1.5 \mathrm{~nm}[97]$. While enhancement of the 
(a)
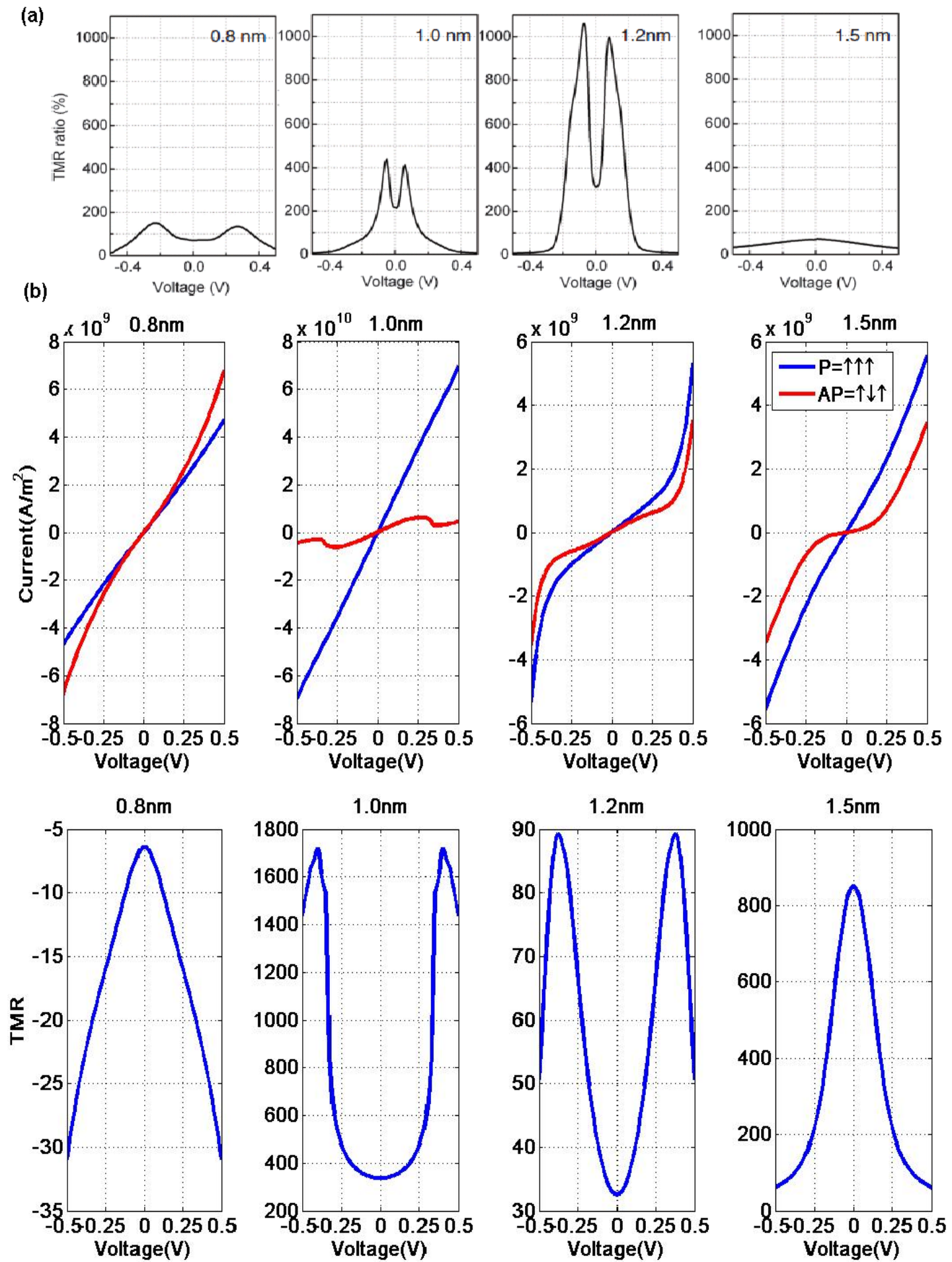

Figure 7.5: (a) Large Tunnel Magnetoresistance of $1048 \%$ at Room Temperature in $\mathrm{MgO}$ Based Double Barrier Magnetic Tunnel Junction, l Jiang et al, Appl. Phys. Express 2 (2009) 083002. (b) Effect on TMR by free layer width modulation around $1 \mathrm{~nm}$ by numerical calculation (Non-Equilibrium Green's Function). 

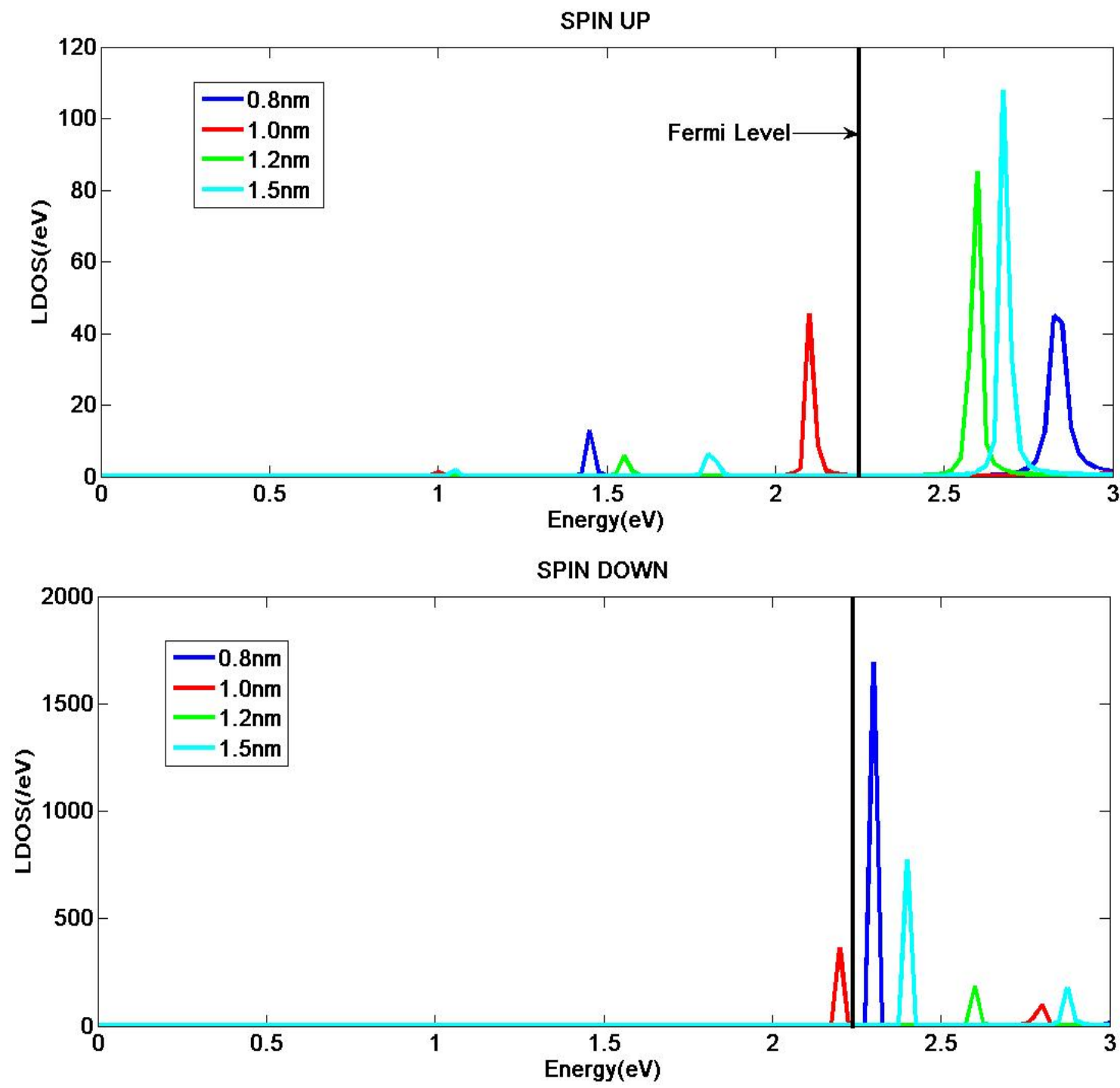

Figure 7.6: Effect on resonance state by free layer width modulation.

TMR to $1048 \%$ at $\mathrm{t}=1.2 \mathrm{~nm}$ is seen, the TMR reduces to $100 \%$ at $\mathrm{t}=1.5 \mathrm{~nm}$. The position of the Van-Hove singularities is dependent on the thickness of the free layer as well the as interfacial properties between the insulators and the free layer. While the TMR enhancement will solve the low TMR issue in DMTJ, whether it would be able to survive manufacturing variance (variation in thickness in free layer,potential barrier, effective electron mass inside the free layer) is a question that remains to be answered.

From our theoretical studies, we can see that a TMR of $1700 \%$ is achieved when free layer width is $1.0 \mathrm{~nm}$ but drops down to $90 \%$ at $1.2 \mathrm{~nm}$ (Fig $7.5 \mathrm{~b}$ ). In-depth study of the resonances show a majority up spin resonance state near the Fermi level at 1nm, while no major peaks at 1.2nm (Fig. 7.6). 


\subsection{Alternate design FM-M-FM-I-FM}

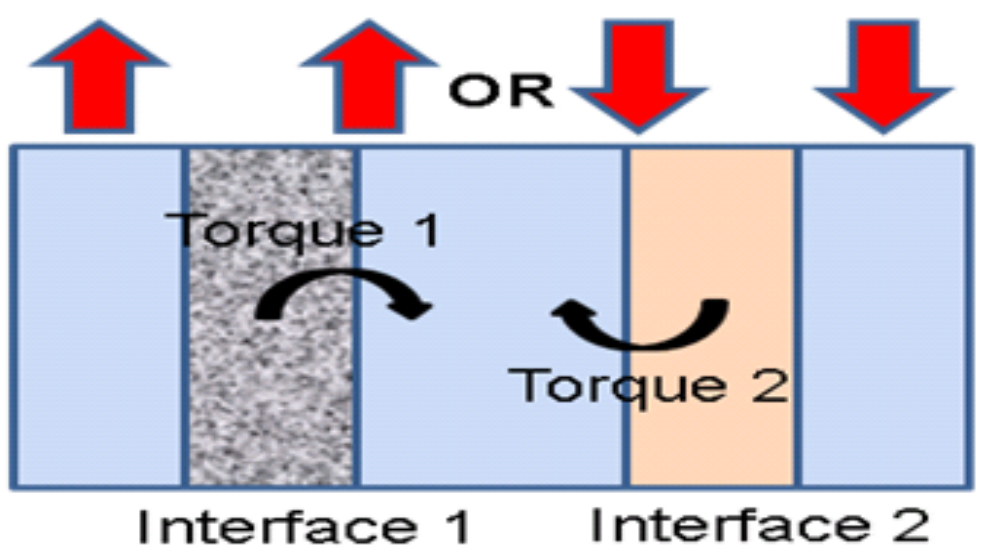

Figure 7.7: Fixed FM-Metal-Free FM-Insulator-Fixed FM system

An alternate way to take advantage of the double spin torque switching mechanism is to employ a Fixed FM-Metal-Free FM-Insulator-Fixed FM system (Fig. 7.2). The above configuration has been seen to reduce the spin torque switching by $\approx 70 \%$ [89]. The TMR is preserved as the electrons have to pass through one tunneling barrier and will be dependent on the Free FM-Insulator-Fixed FM configuration.

\subsection{Combinatorial STT-RAM}

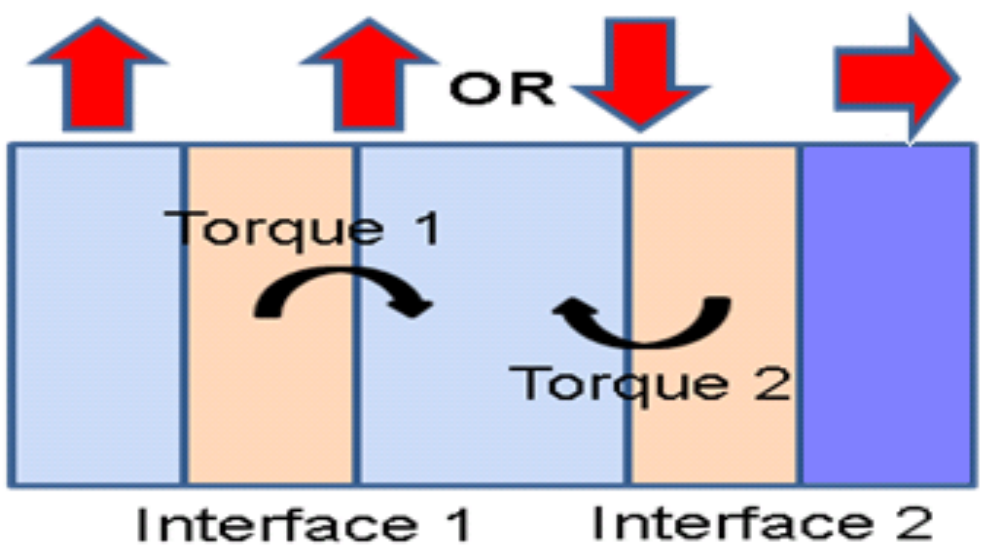

Figure 7.8: Combinatorial STT-RAM

In Combinatorial DMTJ, there will be two torques, one exerted by left contact at interface 1 by the left in-plane fixed layer and the 2nd exerted by right perpendicular contact at interface 2 (Fig 7.8). As torque 
is proportional to $\sin \theta$, the torque exerted by the right perpendicular layer is much stronger than the left in-plane layer, thereby increasing the switching efficiency compared to that in a two in-plane fixed layers DMTJ.

\subsection{A quasi-analytical model to study energy-delay-reliability trade-off in nanomagnetic logic}

A Fokker-Plank solution for the two step switching in nanomagnetic logic (stress+dipole) needs to be worked out to analytically calculate error rate with a given voltage plus and NM separation. Setting up an analytical version of the system will result in faster reliability study without performing a full-fledged stochastic calculation. 


\section{Appendix A}

\section{Modeling magnetic tunnel junction}

\section{using NEGF}

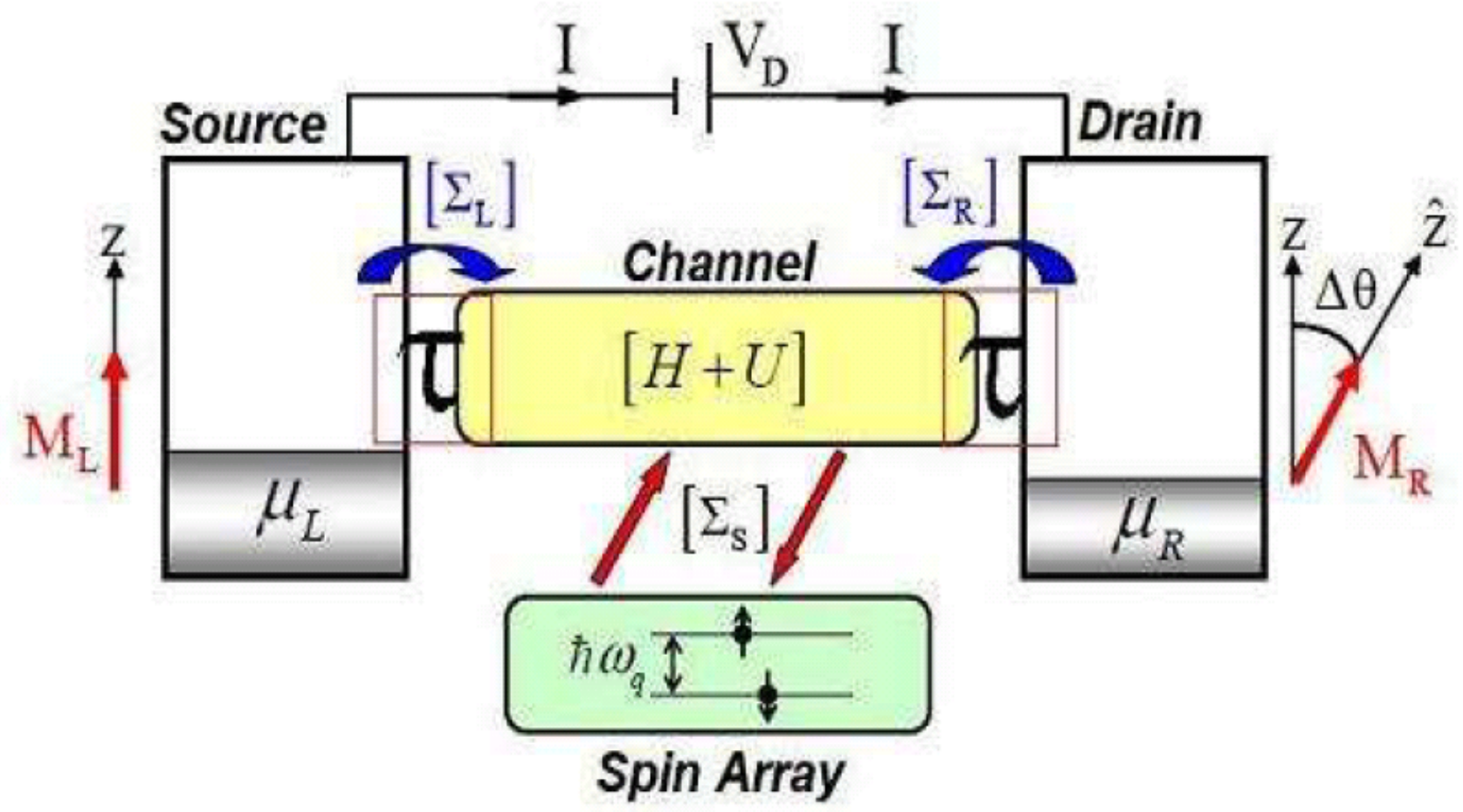

Figure A.1: Schematic illustration of the device partitioning with matrices needed for NEGF quantum transport calculations. Magnetization direction of the drain is defined relative to the $\operatorname{source}\left(\Delta \theta=\theta_{R}-\theta_{L}\right)$

Through the Keldysh-Kadanoff-Baym Non-Equilibrium Green's function (NEGF) method, a device is partitioned into channel and contact regions as illustrated in Fig. A.1. Components of the partitioned device 
can be classified in four categories:

(i) Channel properties are defined by the Hamiltonian matrix $[\mathrm{H}]$ including the applied bias potential and the potential barrier for the insulator. Single band tight-binding approximation is adopted[48]. The single barrier MTJ in a STT-RAM can be specified by the following five parameters[47]: (i) $U_{\text {barr }}$ is the barrier offset between the contact and the insulator, (ii) $E_{F}$ is Fermi-level,(iii) $\delta$ is the band splitting between majority and minority spin electrons, (iV) $\mathrm{W}$ is the width of the insulator, (v) $m_{c}$ and (vi) $m_{\text {barr }}$ are the effective masses in the contact and barrier respectively. In real space, for a discrete lattice whose points are located at $\mathrm{x}=\mathrm{ja}$, $\mathrm{j}$ being an integer $(\mathrm{j}=1 \ldots \mathrm{N})$, the matrix $\mathrm{H}$ can be expressed as:

$$
\begin{aligned}
& \mid 1> \\
& \mid 2> \\
& \mid N-1> \\
& \mid N>
\end{aligned}\left[\begin{array}{ccccc}
\mid 1> & \mid 2> & |\cdots\rangle & \mid N-1> & \mid N> \\
\alpha_{1} & \beta & \cdots & 0 & 0 \\
\beta^{+} & \alpha_{2} & \cdots & 0 & 0 \\
\vdots & \vdots & \ddots & \vdots & \vdots \\
0 & 0 & \cdots & \alpha_{N-1} & \beta \\
M 0 & 0 & \cdots & \beta^{+} & \alpha_{N}
\end{array}\right]
$$

where $\alpha_{n}$ is a $2 \times 2$ spin-based on-site matrix:

$$
\alpha_{n}=\left.\right|_{\mid \downarrow>} ^{\mid \uparrow>}\left[\begin{array}{cc}
\mid \uparrow> & \mid \downarrow> \\
E_{C \uparrow}+2 t+U_{n} & 0 \\
0 & 2 t+E_{C \downarrow}+U_{n}
\end{array}\right]
$$

and $\beta=\mathrm{tI}$ is a $2 \mathrm{x} 2$ site-coupling matrix with $\mathrm{t}=\hbar^{2} / 2 m a^{2}$ and $\mathrm{I}$ is $2 \times 2$ identity matrix. The on-site $U$ consists of the added potential due to applied voltage. For an applied positive voltage V, the Fermi level of the left fixed contact is shifted up by $+q \mathrm{~V} / 2$ while the right free contact is shifted down by $-\mathrm{qV} / 2$. Potential is dropped linearly in the barrier. Inside the barrier, $E_{C \uparrow}$ and $E_{C \downarrow}$ are zero. In the ferromagnetic contact, $E_{C \uparrow}=0$ and $E_{C \downarrow}=\delta$.

(ii)Contacts are included through self-energy matrices $\left[\Sigma_{L}\right] /\left[\Sigma_{R}\right]$ whose anti-hermitian component:

$$
\Gamma_{L, R}(E)=i\left(\Sigma_{L, R}(E)-\Sigma_{L, R}^{\dagger}(E)\right)
$$

describes the broadening due to the coupling to the contact. The broadening matrix can be interpreted as the inverse residence time of the escaping electrons from the device. The corresponding in-scattering/outscattering matrices, which describes the rate at which electrons are scattered in/out of a state, are defined as: 


$$
\begin{gathered}
\Sigma_{L, R}^{i n}(E)=F_{2 D}\left(E-\mu_{L, R}\right) \Gamma_{L, R} \\
\Sigma_{L, R}^{\text {out }}(E)=\left[1-F_{2 D}\left(E-\mu_{L, R}\right)\right] \Gamma_{L, R}
\end{gathered}
$$

where $F_{2 D}(E)$ are the 2-D Fermi function at the two contacts. Using the 2-D Fermi function takes care of the transverse momentum in the $\mathrm{Y}$ and $\mathrm{Z}$ directions. $\mu_{L, R}$ are the Fermi levels. The in/out scattering matrices describes the rate at which electrons are scattered in/out of a state.

$$
F_{2 D}\left(E-\mu_{L, R}\right)=\frac{m_{c} K_{B} T}{2 \pi \hbar^{2}} \ln \left[1+\exp \left(-\left(E-\mu_{L, R}\right) / K_{B} T\right)\right]
$$

The Magnetization of the two contacts is given as a function of $\theta . \theta=0$ indicates spin up configuration while $\theta=\pi$ specifies spin down. The left contact self-energy matrix is nonzero only for the first $2 \times 2$ block:

$$
\Sigma_{L}(E)=\underset{\mid \uparrow>}{\mid \downarrow>}\left[\begin{array}{cc}
\mid \uparrow> & |\downarrow\rangle \\
-t e^{i k_{L}^{\uparrow} a} & 0 \\
0 & -t e^{i k_{L}^{\downarrow} a}
\end{array}\right]
$$

where $E=E_{C}^{\uparrow, \downarrow}+U_{L}+2 t\left(1-\operatorname{cosk}_{L}^{\uparrow, \downarrow} a\right)$. For the right contact only the last block is non-zero:

$$
\Sigma_{R}(E)=\tilde{U}_{\mid \downarrow>}^{\mid \uparrow>}\left[\begin{array}{cc}
\mid \uparrow> & |\downarrow\rangle \\
-t e^{i k_{R}^{\uparrow} a} & 0 \\
0 & -t e^{i k_{R}^{\downarrow} a}
\end{array}\right] \tilde{U}^{\dagger}
$$

where $\tilde{U}$ is the unitary transformation operator needed to obtain right contact self-energy matrix in the left contact magnetization spin basis set, if the polarization direction in two contacts differ by an angle $\Delta \theta$ :

$$
\tilde{U}(\Delta \theta)=\left[\begin{array}{cc}
\cos (\Delta \theta / 2) & \sin (\Delta \theta / 2) \\
-\sin (\Delta \theta / 2) & \cos (\Delta \theta / 2)
\end{array}\right]
$$

The Greens function of the device in the coherent regime is:

$$
G=\left(E I-H-\Sigma_{L}-\Sigma_{R}\right)^{-1}
$$

where

$$
E I-H-\Sigma_{L}-\Sigma_{R}=
$$




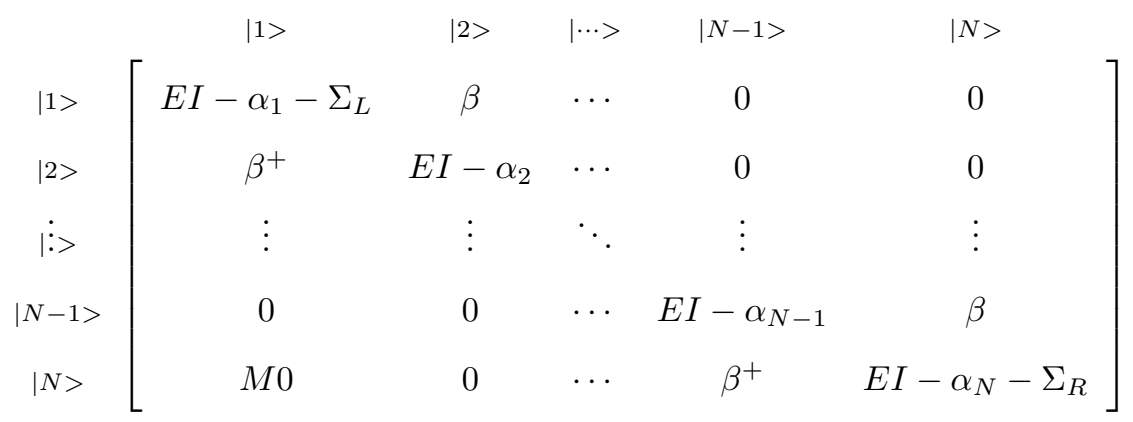

Current through the entire device is calculated by:

$$
I=\frac{q}{\hbar} \int_{-\infty}^{\infty} \operatorname{Trace}\left[\Gamma_{L} G \Gamma_{R} G^{\dagger}\right]\left(F_{2 D}^{L}-F_{2 D}^{R}\right) d E
$$

Adding scattering: Most theoretical work on spin devices use coherent Landauer formula for electron flow, disregarding dephasing, an essential ingredients for STT-MRAM behavior. Spin exchange scattering processes are responsible for the incoherent nature of the tunneling transport for the model devices considered here. What makes this process incoherent are the external forces forcing the impurity spins into local equilibrium, erasing the polarized information of the surrounding through to lose its polarization. We assume that the equilibrium restoring processes are fast enough to maintain the impurity spins in a thermal equilibrium state and are degenerate.

The Keldysh-Kadanoff-Baym Nonequilibrium Green's function (NEGF) approach can handle dephasing by introducing a self-energy for incoherent processes, calculated for a given scattering Hamiltonian (usually invoking a perturbative Born Approximation). Incoherent scattering processes in the channel region are described by a third contact with in/out-scattering matrices $\left[\Sigma_{S}^{i n}\right] /\left[\Sigma_{S}^{o u t}\right]$. Broadening due to scattering is given by:

$$
\Gamma_{S}(E)=\Sigma_{S}^{\text {in }}(E)+\Sigma_{S}^{o u t}(E)
$$

from which the self-energy matrix $\Sigma_{S}$ is obtained through Hilbert transform of $\Gamma_{S}$. The Green's function is defined as:

The Greens function of the device in the coherent regime is:

$$
G=\left(E I-H-\Sigma_{L}-\Sigma_{R}-\Sigma_{S}\right)^{-1}
$$

The electron/hole correlation functions (whose diagonal elements are the electron/hole density) is given by: 


$$
G^{n / p}=G\left[\Sigma_{L}^{\text {in/out }}+\Sigma_{R}^{\text {in/out }}+\Sigma_{S}^{\text {in/out }}\right] G^{\dagger}
$$

The in/out-scattering matrices $\left[\sum_{S}^{i n}\right] /\left[\Sigma_{S}^{o u t}\right]$ are related to the electron/hole correlation functions $\left[G^{n}\right] /\left[G^{p}\right]$ through:

$$
\begin{aligned}
& \sum_{S ; \sigma_{i} \sigma_{j}}^{i n}=D_{\sigma_{i} \sigma_{j} ; \sigma_{k} \sigma_{l}}^{n} G_{\sigma_{k} \sigma_{l}}^{n} \\
& \sum_{S ; \sigma_{i} \sigma_{j}}^{\text {out }}=D_{\sigma_{i} \sigma_{j} ; \sigma_{k} \sigma_{l}}^{p} G_{\sigma_{k} \sigma_{l}}^{p}
\end{aligned}
$$

where $\left[G^{n}\right] /\left[G^{p}\right]$ are electron/hole correlation function, $\left[D^{n}\right] /\left[D^{p}\right]$ are fourth-order scattering tensors. (k,l) and $(\mathrm{i}, \mathrm{j})$ are initial and final spin indexes, respectively. The scattering process is dependent on the initial wave-function and vacancy of the final state. The spin subspace is defined by the following $2 \times 2$ matrix where $\mathrm{i}, \mathrm{k}$ are row indexes and $\mathrm{l}, \mathrm{j}$ are column indexes.

$$
\left[\begin{array}{cc}
\uparrow \uparrow & \uparrow \downarrow \\
\downarrow \uparrow & \downarrow \downarrow
\end{array}\right]
$$

These scattering tensors can be obtained from the spin scattering interaction Hamiltonian,

$$
H=J \vec{\sigma} \cdot \vec{S}
$$

where $\sigma$ and $\mathrm{S}$ are the spin operators for the channel electron/magnetic- impurity respectively and $\mathrm{J}$ is the coupling parameter.

The in/out-scattering matrices $\left[\Sigma_{S}^{i n}\right] /\left[\Sigma_{S}^{o u t}\right]$ and electron/hole correlation functions $\left[G^{n}\right] /\left[G^{p}\right]$ depend on each other, requiring an iterative self-consisted solution of the NEGF equations[98].

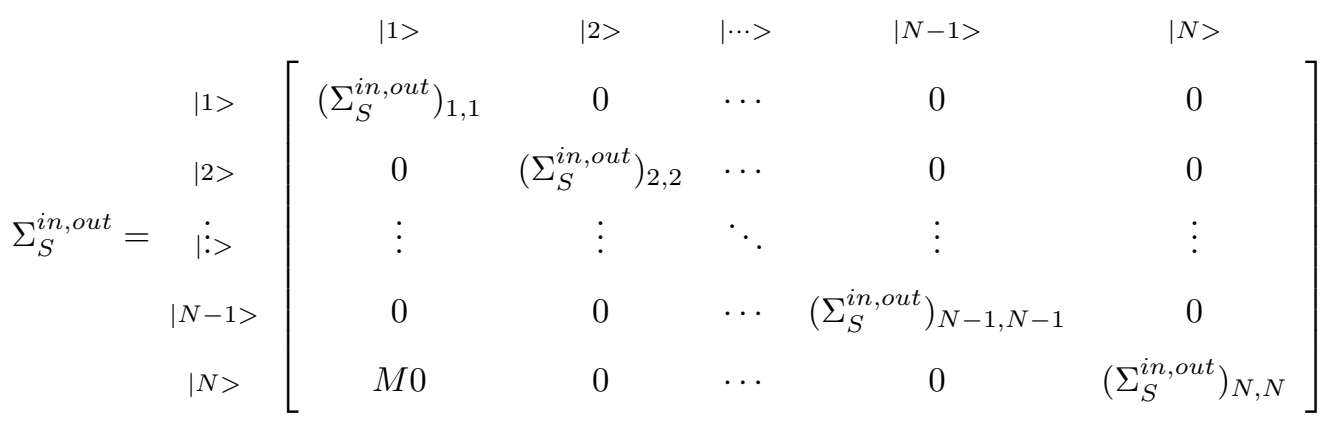




$$
\left[\begin{array}{l}
\left(\Sigma_{S ; \uparrow \uparrow}^{\text {in }, \text { out }}\right)_{j, j} \\
\left(\Sigma_{S ; \downarrow \downarrow}^{\text {in, out }}\right)_{j, j} \\
\left(\Sigma_{S ; \uparrow \downarrow}^{\text {in,out }}\right)_{j, j} \\
\left(\Sigma_{S ; \downarrow \uparrow}^{\text {in,out }}\right)_{j, j}
\end{array}\right]=J^{2} N_{I}\left[\begin{array}{cccc}
0 & F_{u, d} & 0 & 0 \\
F_{d, u} & 0 & 0 & 0 \\
0 & 0 & 0 & 0 \\
0 & 0 & 0 & 0
\end{array}\right]\left[\begin{array}{l}
\left(G_{\uparrow \uparrow}^{n, p}\right)_{j, j} \\
\left(G_{\downarrow \downarrow}^{n, p}\right)_{j, j} \\
\left(G_{\uparrow \downarrow, p}^{n, p}\right)_{j, j} \\
\left(G_{\downarrow \uparrow}^{n, p}\right)_{j, j}
\end{array}\right]
$$

where $N_{I}$ is the number of magnetic impurities and $F_{u} / F_{d}$ represents fractions of spin-up/spin-down impurities for an uncorrelated ensemble $\left(F_{u}=0.5\right.$ and $\left.F_{d}=0.5\right)$. Simplifying the tensor relationship, the in/out-scattering into spin-up component is proportional to the density of the spin-down electrons/holes times the number of spin up impurities, $N_{I} F_{u}$ :

$$
\left(\sum_{S, \uparrow, \uparrow}^{\text {in out }}\right)_{j, j}=J^{2} N_{I} F_{u}\left(G_{\downarrow, \downarrow}^{n, p}\right)_{j, j}
$$

Similarly, the in/out-scattering into spin-down component is proportional to the density of the spin-up electrons/holes times the number of spin down impurities, $N_{I} F_{d}$ :

$$
\left(\sum_{S, \downarrow, \downarrow}^{\text {in,out }}\right)_{j, j}=J^{2} N_{I} F_{d}\left(G_{\uparrow, \uparrow}^{n, p}\right)_{j, j}
$$

Fig A.2 shows the effect of incoherent scattering on tunneling magnetoresistance for the $\mathrm{CoFeB} / \mathrm{MgO} / \mathrm{CoFeB}$ parameters from chapter 2 . 


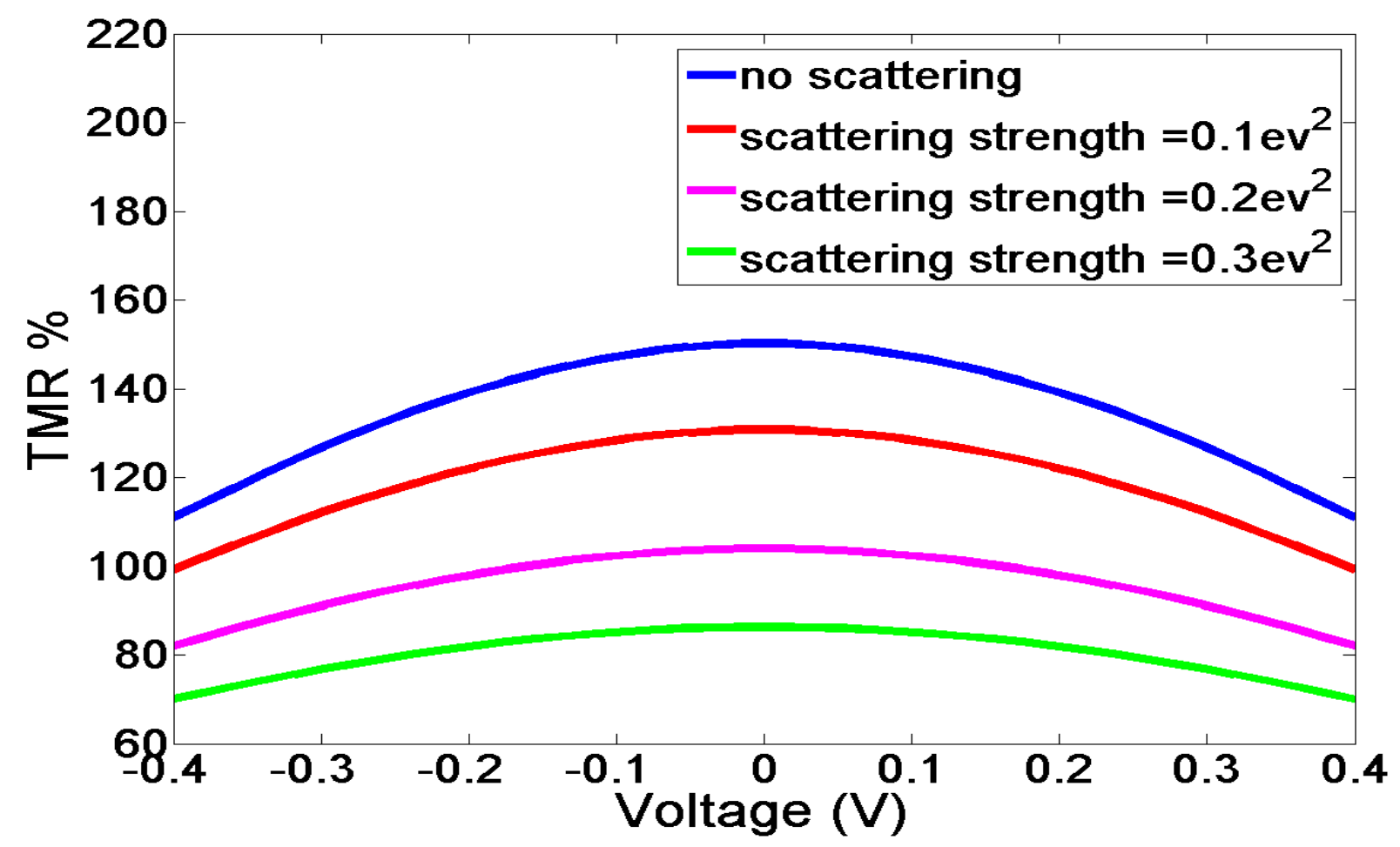

Figure A.2: Effect of inelastic scattering on tunneling magnetoresistance. 


\section{Appendix B}

\section{Atomistic study of strained}

\section{$\mathrm{Fe} / \mathrm{MgO} / \mathrm{Fe}$}

\section{B.1 Extraction of Extended Huckel theory parameters from VASP bandstructure}

A first-principles calculation using density functional theory(DFT) within the generalized gradient approximation (GGA) implemented in VASP is used to calculate the electronic structure of Iron and Iron-Magnesium oxide heterostructure. These bandstructures are then used to determine the parameters of an Extended Huckel tight-binding model (EHT-TB) that accurately reproduces the bands. The above method is much faster that first-principles method and can deal with a very dense k-points mesh without exhausting computational recourses. The parameter fitting and extraction was done by Chunsheng Liu at University of Alabama and details can be found at [99]. EHT-TB parameters for spin up and down strained bulk Fe and Fe-MgO interface are given in Figs. B.1-B.4.

\section{B.2 Strained Fe/MgO/Fe tunnel junction}

The strained $\mathrm{Fe} / \mathrm{MgO} / \mathrm{Fe}$ magnetic tunnel junction is broken into three parts: the left and right semi-infinite ferromagnetic contact and the middle channel which hosts the tunneling barrier (Fig. B.5). The device is xy periodic in [100] plane of both $\mathrm{MgO}$ and $\mathrm{Fe}$ and transport takes place in the z direction. The two ferromagnetic leads extend to reservoirs at $\mathrm{z}= \pm \infty$. The central channel is chosen sufficiently large in the $\mathrm{z}$ direction such that: (i) the potentials outside the central region are taken as equivalent to bulk and (ii) the 
BCFe

2.9783 \#The lattice constant $-\rightarrow \mathrm{a} 0$

1 \#the spin index -->ispin; spin-polarized fitting:1; with SO:2;

1.5 \#the cutoff distance for hopping neighbors -.> cut_off

1 H how many kinds of atoms in one unitcell-., nkind

100.06200 \#SO coupling number; Not used in spin polarized case, but keep it

The number of atomic orbitals included in the fitting.

9 HFe | ->n_orbital[nkind,ispin]

Fe-Fe

1.696675

0 H-.>k_H_freez

The correspondence between magnetic quantum nubmer and atomic orbitals are:

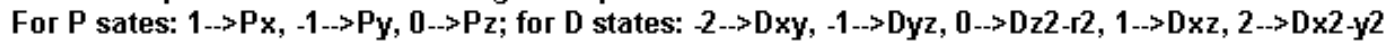

The ETH parameters:

STO_name NZ N L M ZETA1 free ZETA2 free H_onsite free COEF1 free Up SPIN PARAMETERS

$\begin{array}{lccccccccccccc}\text { uFe_s } & 26 & 4 & 0 & 0 & 1.818574 & 11 & 24.523128 & 21 & -18.164304 & 31 & 1.000000 & 1 & 0.000000 \\ \text { uFe_px } & 26 & 4 & 1 & 1 & 1.744312 & 12 & 24.523128 & 22 & -13.263144 & 32 & 1.000000 & 2 & 0.000000 \\ \text { uFe_py } & 26 & 4 & 1 & -1 & 1.744312 & 12 & 24.523128 & 22 & -13.263144 & 32 & 1.000000 & 2 & 0.000000 \\ \text { uFe_pz } & 26 & 4 & 1 & 0 & 1.744312 & 12 & 24.523128 & 22 & -13.263144 & 32 & 1.000000 & 2 & 0.000000 \\ \text { uFe_dxy } & 26 & 3 & 2 & -2 & 24.999664 & 13 & 1.972344 & 23 & -21.864856 & 33 & 0.840199 & 43 & 0.533630 \\ \text { uFe_dyz } & 26 & 3 & 2 & -1 & 24.999664 & 13 & 1.972344 & 23 & -21.864856 & 33 & 0.840199 & 43 & 0.533630 \\ \text { uFe_dzx } & 26 & 3 & 2 & 1 & 24.999664 & 13 & 1.972344 & 23 & -21.864856 & 33 & 0.840199 & 43 & 0.533630 \\ \text { uFe_dx2 } & 26 & 3 & 2 & 2 & 24.999664 & 13 & 1.972344 & 23 & -21.864856 & 33 & 0.840199 & 43 & 0.533630\end{array}$

Figure B.1: Parameters for contact spin up Fe.

BCFe

2.9783 \#The lattice constant $-\rightarrow \mathrm{a} 0$

1 \#the spin index -->ispin; spin-polarized fitting:1; with S0:2;

1.5 \#the cutoff distance for hopping neighbors -.>cut_off

1 \# how many kinds of atoms in one unitcell-.> nkind

100.06200 \#SO coupling number; Not used in spin polarized case, but keep it

The number of atomic orbitals included in the fitting.

9 \#Fe | -.>n_orbital[nkind, ispin]

$\mathrm{Fe}-\mathrm{Fe}$

1.857209

0 H-.>k_H_freez

The correspondence between magnetic quantum nubmer and atomic orbitals are:

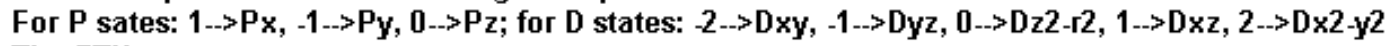

The ETH parameters:

ST0_name NZ N L M ZETA1 free ZETA2 free H_onsite free COEF1 free Down SPIN PARAMETERS

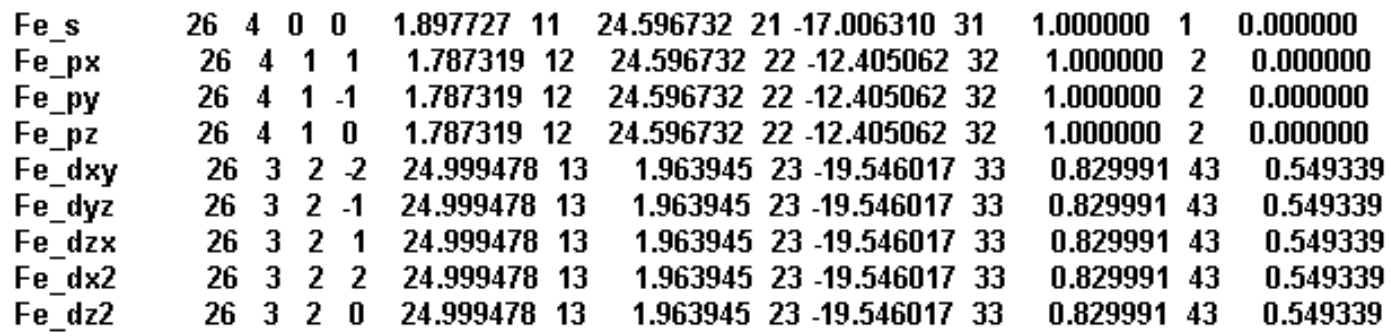

Figure B.2: Parameters for contact spin down Fe. 


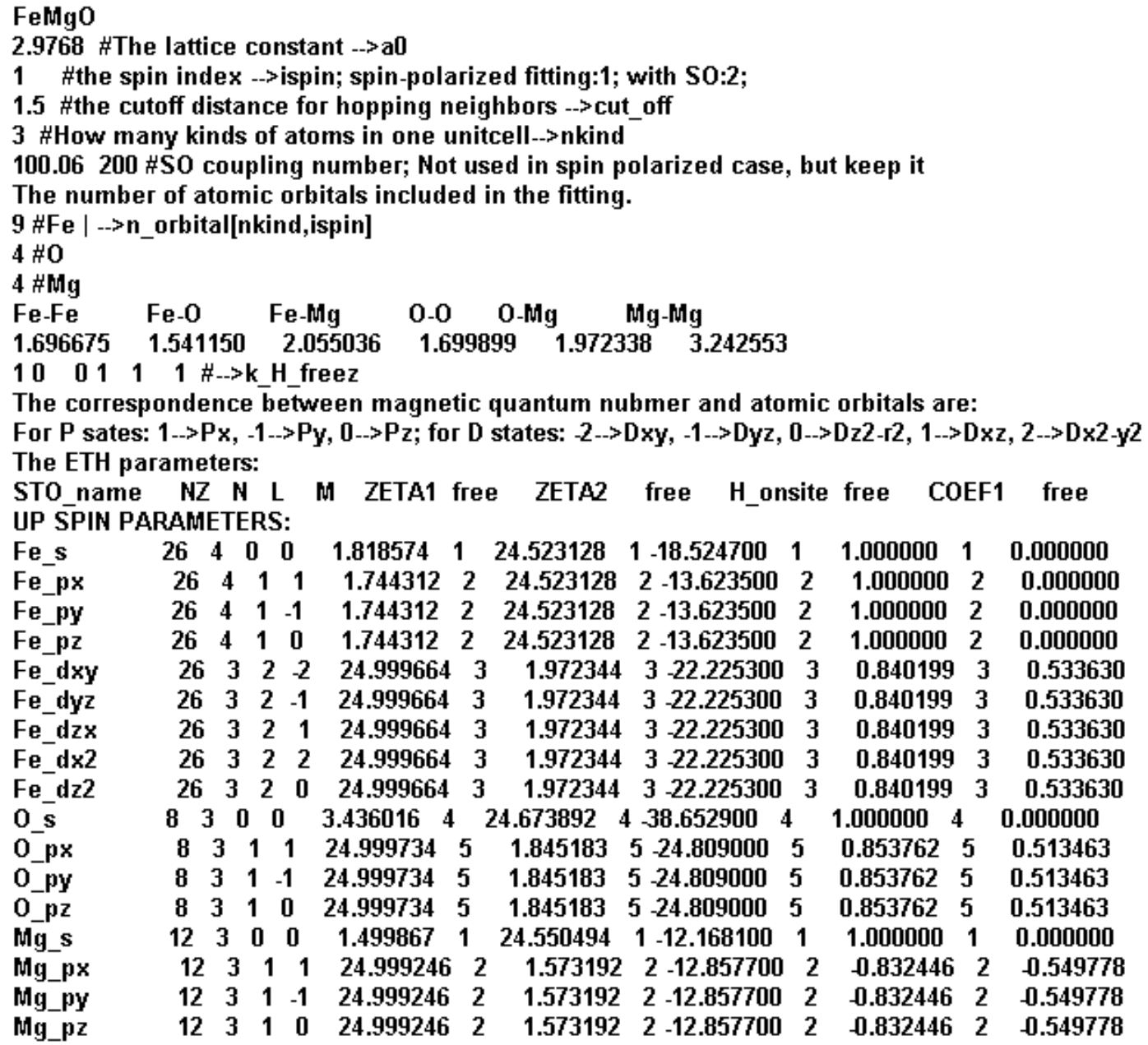

Figure B.3: Parameters for spin up strained Fe/5 [100] layers of $\mathrm{MgO} / \mathrm{Fe}$ magnetic tunneling junction.

matrix elements coupling the left and right leads are zero. The electrochemical potentials of the left and right leads, $\mu_{L}$ and $\mu_{R}$, are given by the bulk Fermi level of the ferromagnets that can be calculated by DFT at equilibrium, and the applied external bias voltage.

Because the device is $\mathrm{x}-\mathrm{y}$ periodic, the eigenstates of the system can be labeled according to their transverse momentum:

$$
\Psi^{k_{\|}}\left(R_{\|}+r\right)=e^{i k_{\|} \cdot R_{\|}} \times e^{i k_{\|} \cdot r} \phi^{k_{\|}}(r)
$$

where $k_{\|}$is a Block wavevector, $R_{\|}=n_{x} a+n_{y} b$ is the lattice vector and $\phi^{k_{\|}}$is the x-y Block function. Using the Bloch ansatz, the Schrödinger equation can be written in the matrix form as:

$$
\tilde{H}^{k_{\|}} \phi^{k_{\|}}=E \tilde{S}^{k_{\|}} \phi^{k_{\|}}
$$

where $\tilde{H}^{k_{\|}}$and $\tilde{S}^{k} \|$ is the folded Hamiltonian and overlap matrix defined as: 


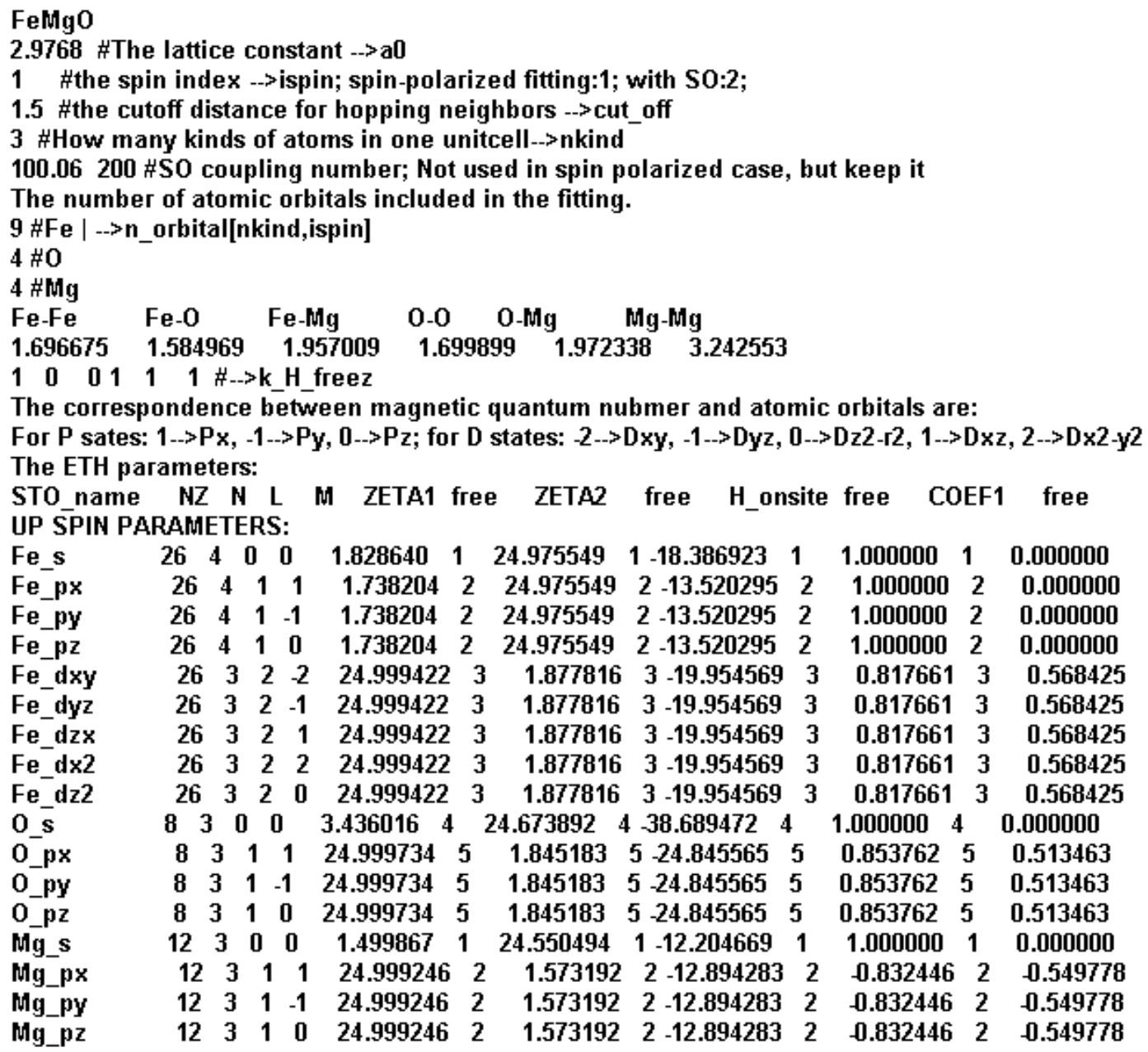

Figure B.4: Parameters for spin down strained $\mathrm{Fe} / 5$ [100] layers of $\mathrm{MgO} / \mathrm{Fe}$ magnetic tunneling junction.

$$
\begin{aligned}
\tilde{H}^{k_{\|}} & =\sum_{n_{x}, n_{y}} H_{n_{x}, n_{y}} e^{i k_{\|} \cdot R_{\|}} \\
\tilde{S}^{k_{\|}} & =\sum_{n_{x}, n_{y}} S_{n_{x}, n_{y}} e^{i k_{\|} \cdot R_{\|}}
\end{aligned}
$$

In this equation, $H_{n_{x}, n_{y}}$ is the Hamiltonian matrix connecting two unit cells separated by $R_{\|}$.

\section{B.3 Fe $\Sigma$ calculation of $[100]$ surface}

The main point in the surface $\Sigma$ calculation is that in the 2-D surface the crystal is infinite and periodic, but semi-infinite in the third depth direction where it's truncated on one side. We will Fourier transform our structure in the in-plane direction, so that we get a k-space bandstructure for the in-plane part ( $\mathrm{k}$ is 


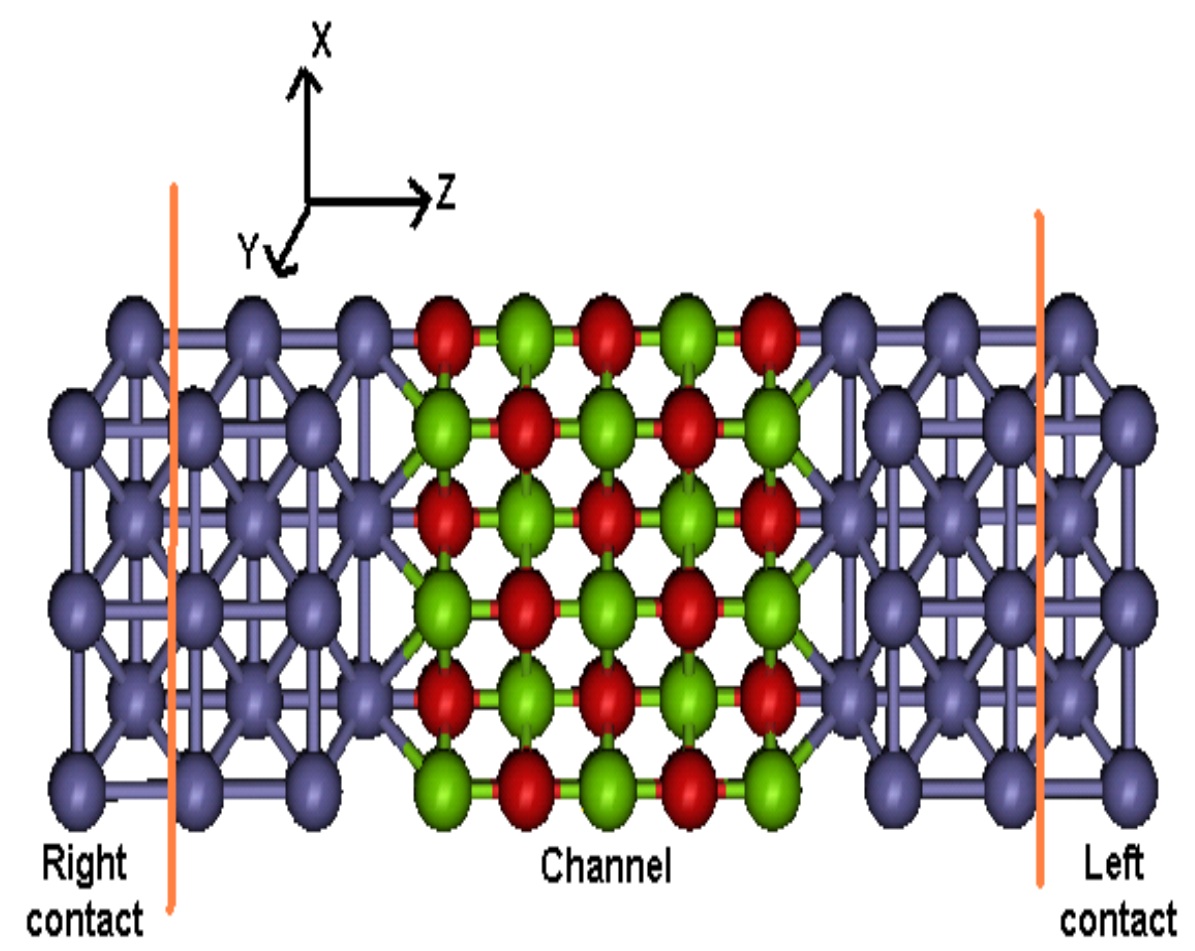

Figure B.5: Atomistic view of a strained $\mathrm{Fe} / 5$ [100] layers of $\mathrm{MgO} / \mathrm{Fe}$ magnetic tunneling junction. The system is periodic in the $\mathrm{x}-\mathrm{y}$ plane. Current flows in the $\mathrm{Z}$ direction.

a 2 -D vector). Then each $\mathrm{k}$ point acts like an independent point in the 2 -D plane, meaning that it acts as an independent 1-D semi-infinite solid in the third direction. We can calculate the surface Green's function of each of these solids, and then inverse transform to get back the real-space surface Green's function at a desired surface atom.

Let's first define the crystal structure so as to identify its 2-D in-plane reciprocal lattice vectors and define the discrete k-points for 2-D ring boundary conditions. Fig. B.6 shows the [100] plane of the BCC unit cell of Fe. The unit cell includes an atom in-plane and the body-centered atom in the next plane (highlighted in red). The green boxes indicate the next nearest neighbors. there are 27 unit cells that we will need in a nearest-neighbor theory. These atoms include the 9 unit cells in the in-plane shown in Fig. B.6, two atomic planes (each containing 9 unit cells) before and after the main plane. All the atomic points are used to calculate the $H_{k_{\|}}^{o n}, H_{k_{\|}}^{o f f}, S_{k_{\|}}^{o n}$ and $S_{k_{\|}}^{\text {off }}$ where "on" now represents the plane and "off" represents the neighboring planes (Fig. B.7). The point is that the k-space squeezes each plane to a point effectively like an "atom". In units of the Fe lattice parameter, these 27 coordinates ( $\mathrm{x}, \mathrm{y}$ only, $\mathrm{z}$ is unimportant for the Fourier-transform which is only done in 2-D) are:

On-site plane: $\mathrm{R} 1=\left[\begin{array}{ll}0 & 0\end{array}\right], \mathrm{R} 2=\left[\begin{array}{ll}1 & 0\end{array}\right], \mathrm{R} 3=\left[\begin{array}{ll}0 & 1\end{array}\right], \mathrm{R} 4=\left[\begin{array}{ll}-1 & 0\end{array}\right], \mathrm{R} 5=\left[\begin{array}{ll}0 & -1\end{array}\right], \mathrm{R} 6=\left[\begin{array}{ll}1 & 1\end{array}\right], \mathrm{R} 7=[-11], \mathrm{R} 8=\left[\begin{array}{ll}-1 & -1\end{array}\right], \mathrm{R} 9=[1 ;-1]$ 


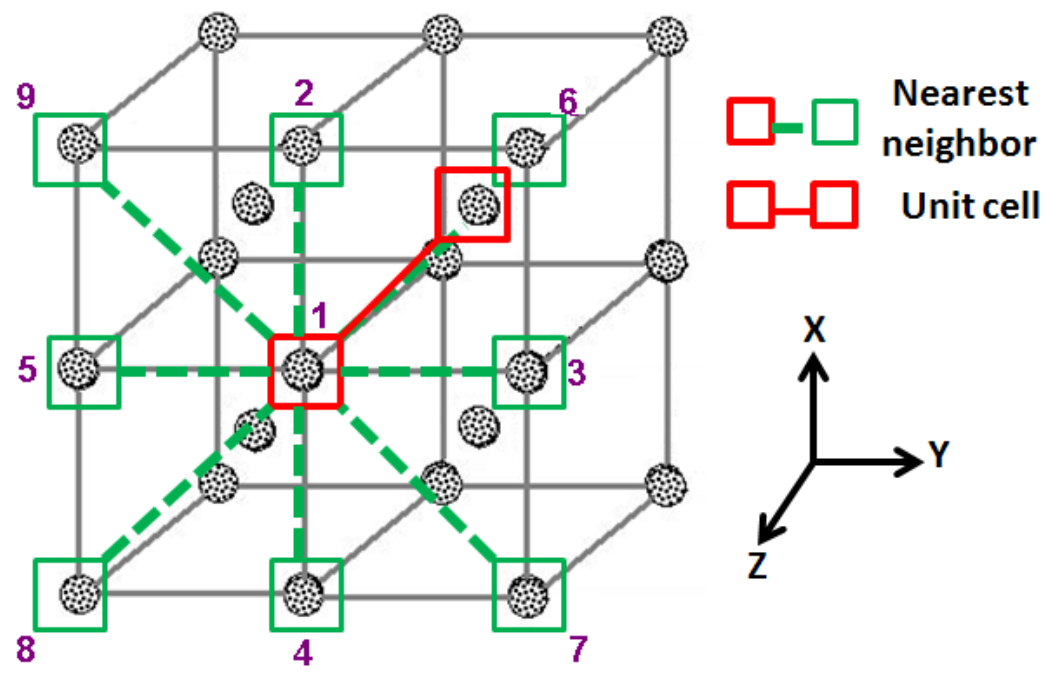

Figure B.6: [100] plane of Fe. Green box indicate the nearest neighbors. The red-red block represent the unit cell.

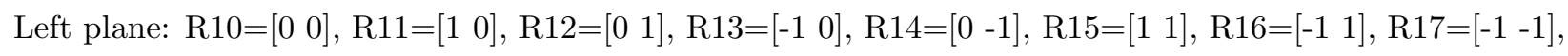
$\mathrm{R} 18=[1 ;-1]$

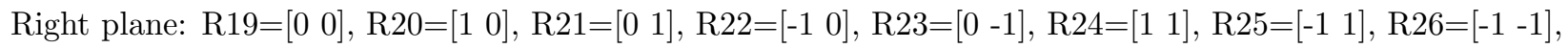
$\mathrm{R} 27=[1 ;-1]$

The reciprocal lattice vectors are: $K_{1}=2 \pi\left[\begin{array}{ll}0 & 1\end{array}\right]$ and $K_{2}=2 \pi\left[\begin{array}{ll}1 & 0\end{array}\right]$.

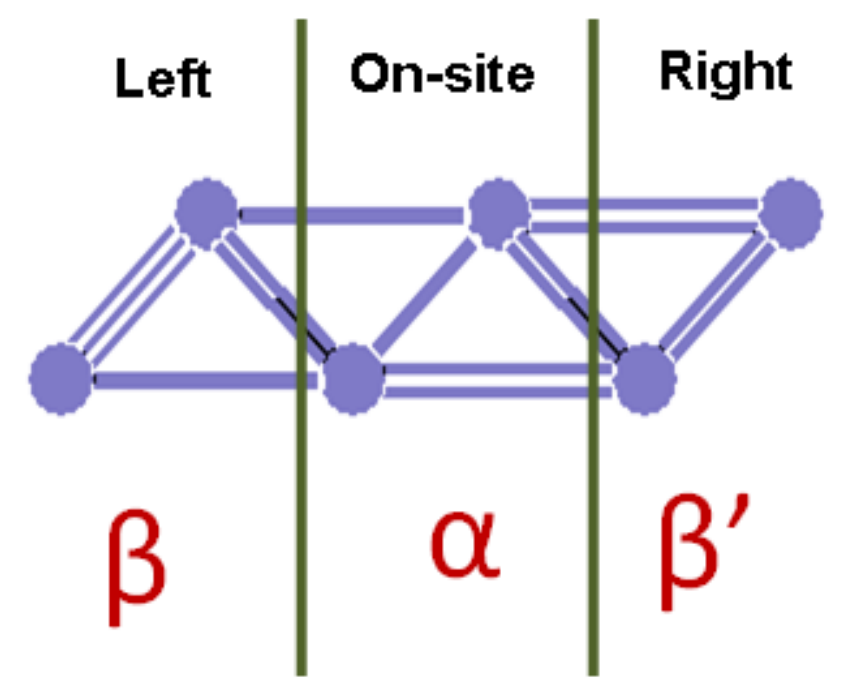

Figure B.7: On-site and neighboring unit cells during contact Fe $\Sigma$ calculation.

Let's calculate the $H_{k_{\|}}^{o n}, H_{k_{\|}}^{o f f}, S_{k_{\|}}^{o n}$ and $S_{k_{\|}}^{o f f}$ : 


\section{$0.0000000 .0000000 .000000 \mathrm{Fe}$ $1.4884001 .4884001 .399400 \mathrm{Fe}$ $0.0000000 .0000002 .798800 \mathrm{Fe}$ $1.4884001 .4884004 .198200 \mathrm{Fe}$ $0.0000000 .0000005 .597600 \mathrm{Fe}$ $1.4884001 .4884006 .997000 \mathrm{Fe}$}

Figure B.8: On-site atomic coordinates for contact Fe $\Sigma$ calculation in Fig. B.7.

$$
\begin{aligned}
H_{k_{\|}}^{o n} & =\sum_{a=1}^{9} H_{1, a} e^{i k_{\|} \cdot R_{a}} \\
S_{k_{\|}}^{o n} & =\sum_{a=1}^{9} S_{1, a} e^{i k_{\|} \cdot R_{a}} \\
H_{k_{\|}}^{o f f} & =\sum_{a=10}^{8} H_{1, a} e^{i k_{\|} \cdot R_{a}} \\
S_{k_{\|}}^{o f f} & =\sum_{a=10}^{8} S_{1, a} e^{i k_{\|} \cdot R_{a}}
\end{aligned}
$$

We do not need to calculate $H_{\text {off }}$ and $S_{\text {off }}$ for right plane as it is just the hermitian of the left one. The Green's function for the semi-infinite system is:

$$
G_{k_{\|}}=\left[\begin{array}{lllll}
\ddots & & & \\
& \alpha_{k_{\|}} & \beta_{k_{\|}} & \\
& \beta_{k_{\|}}^{\dagger} & \alpha_{k_{\|}} & \\
& & & \ddots
\end{array}\right]
$$

where,

$$
\begin{gathered}
\alpha_{k_{\|}}=E S_{k_{\|}}^{o n}-H_{k_{\|}}^{o n} \\
\beta_{k_{\|}}=E S_{k_{\|}}^{o f f}-H_{k_{\|}}^{o f f}
\end{gathered}
$$

We need the "surface" Green's function, which means after inverting the infinite matrix above, we need to evaluate the first $(1,1)$ th block. This means that the surface Green's function, $g_{k_{\|}}$, is 
B.3 $\mid$ Fe $\Sigma$ calculation of [100] surface

$$
g_{k_{\|}}=\left(\alpha_{k_{\|}}-\beta_{k_{\|}} g_{k_{\|}} \beta_{k_{\|}}^{\dagger}\right)^{-1}
$$

The above recursive equation can be solved by the accelerated method specified in [100]. Surface $\Sigma$ and density of states is calculated by,

$$
\begin{gathered}
\Sigma_{k_{\|}}=\beta_{k_{\|}} g_{k_{\|}} \beta_{k_{\|}^{\dagger}}^{\dagger} \\
D O S_{k_{\|}}^{\text {surface }}=\frac{i}{2 \pi}\left(g_{k_{\|}} S_{k_{\|}}^{\text {on }}-S_{k_{\|}}^{\text {on }} g_{k_{\|}}^{\dagger}\right)
\end{gathered}
$$

The bulk density of states can be calculated by:

$$
\begin{gathered}
G_{k_{\|}}=\left(\alpha_{k_{\|}}-2 \Sigma_{k_{\|}}\right)^{-1} \\
D O S_{k_{\|}}^{b u l k}=\frac{i}{2 \pi}\left(g_{k_{\|}} S_{k_{\|}}^{o n}-S_{k_{\|}}^{o n} g_{k_{\|}}^{\dagger}\right)
\end{gathered}
$$

Figs. B.9 and B.10 shows the calculated bulk density of states with EHT-TB parameters and the results are in good agreement with bulk density of states calculated by VASP.

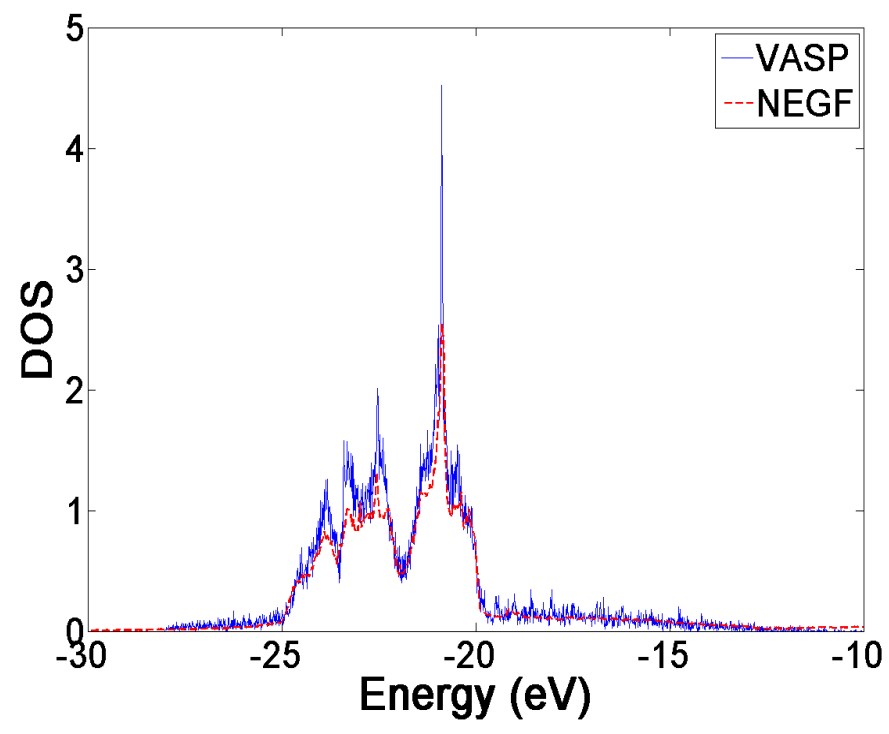

Figure B.9: Density of states for contact spin up Fe. 


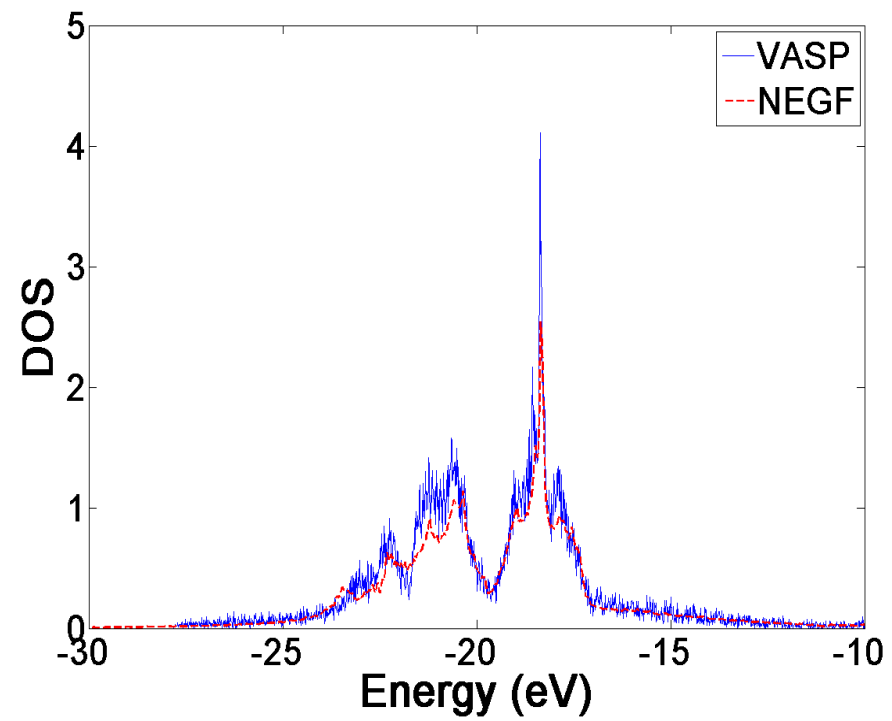

Figure B.10: Density of states for contact spin down Fe.

\section{B.4 Current calculation}

Fig. B.11 shows the coordinates of strained $\mathrm{Fe} / \mathrm{MgO} / \mathrm{Fe}$ magnetic tunnel junction. Because of the lattice mismatch between the $\mathrm{Fe}$ and $\mathrm{MgO}$, the $\mathrm{Fe}$ atoms near the $\mathrm{MgO}$ interface are strained out and does not match that of lead Fe. Taking the right coordinates is essential for the problem as it will determine the correct interfacial chemistry between the contacts and the insulator. Following the prescription similar to that used to calculate $\Sigma_{k_{\|}}$, we can calculate current through the tunnel junction by:

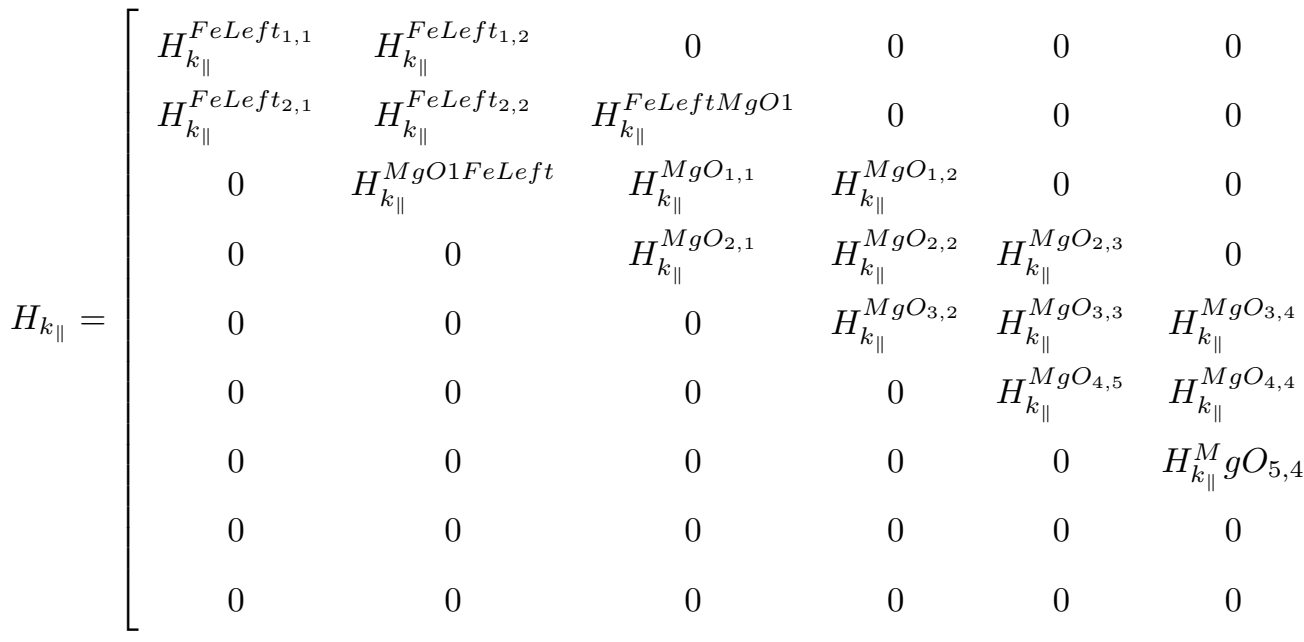




$\left.\begin{array}{ccc}0 & 0 & 0 \\ 0 & 0 & 0 \\ 0 & 0 & 0 \\ 0 & 0 & 0 \\ 0 & 0 & 0 \\ H_{k_{\|}}^{M g O_{4,5}} & 0 & 0 \\ H_{k_{\|}}^{M g O_{5,5}} & H_{k_{\|}}^{M g O 5 F e R i g h t} & 0 \\ H_{k_{\|}^{F e R i g h t M g O 5}}^{F} & H_{k_{\|}}^{F e R i g h t_{1,1}} & H_{k_{\|}}^{F e R i g h t_{1,2}} \\ 0 & H_{k_{\|}}^{F e R_{i}} & H_{k_{\|}}^{F e R i g h t_{2,2}}\end{array}\right]$

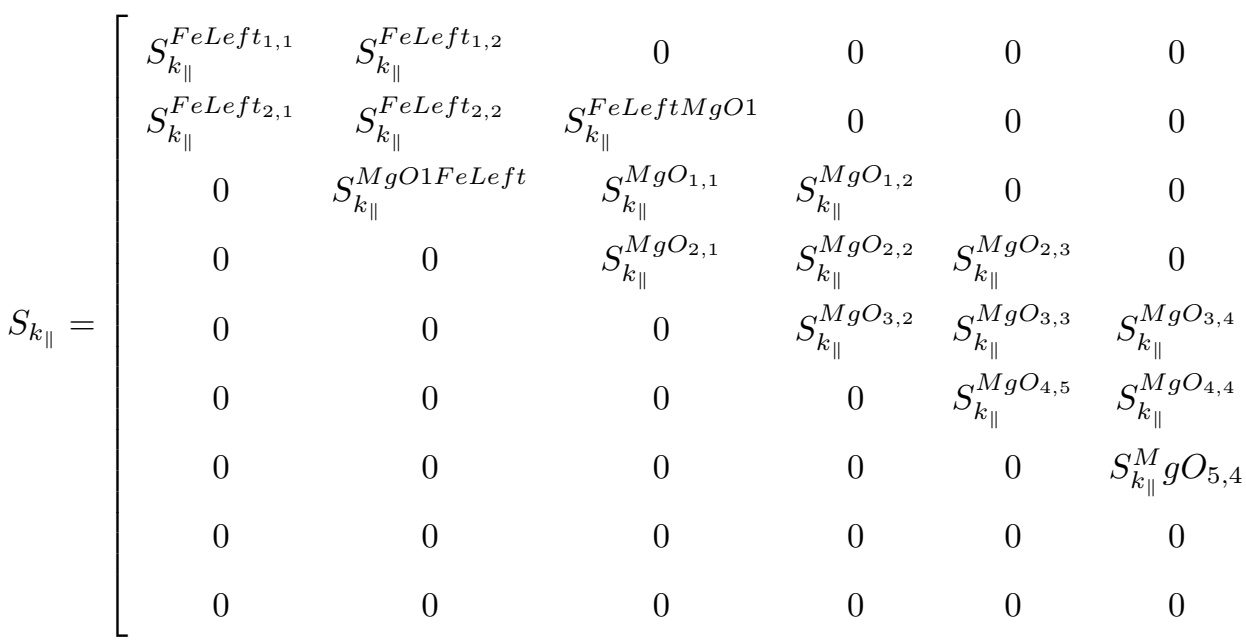

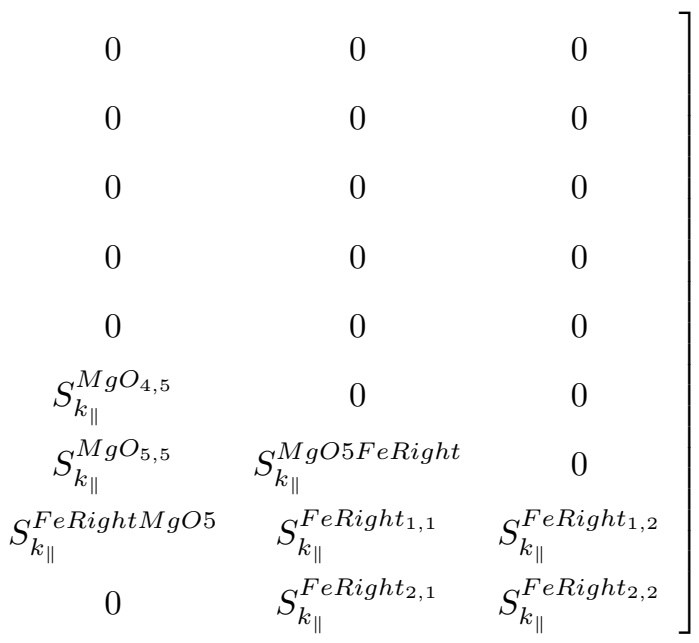




$$
\begin{aligned}
& U_{k_{\|}}(V)=V\left[\begin{array}{ccccc}
0.5 S_{k_{\|}}^{\text {FeLeft } t_{1,1}} & 0.5 S_{k_{\|}}^{\text {FeLeft }} t_{1,2} & 0 & 0 & 0 \\
0.5 S_{k_{\|}}^{\text {FeLeft } t_{2,1}} & 0.5 S_{k_{\|}}^{\text {FeLeft }} t_{2,2} & 0.5 S_{k_{\|}}^{\text {FeLeftMgO1 }} & 0 & 0 \\
0 & 0.5 S_{k_{\|}}^{M g O 1 F e L e f t} & 0.5 S_{k_{\|}}^{M g O_{1,1}} & 0.375 S_{k_{\|}}^{M g O_{1,2}} & 0 \\
0 & 0 & 0.375 S_{k_{\|}}^{M g O_{2,1}} & 0.25 S_{k_{\|}}^{M g O_{2,2}} & 0.125 S_{k_{\|}}^{M g O_{2,3}} \\
0 & 0 & 0 & 0.125 S_{k_{\|}}^{M g O_{3,2}} & 0.0 S_{k_{\|}}^{M g O_{3,3}} \\
0 & 0 & 0 & 0 & -0.125 S_{k_{\|}}^{M g O_{4,5}} \\
0 & 0 & 0 & 0 & 0 \\
0 & 0 & 0 & 0 & 0
\end{array}\right. \\
& \left.\begin{array}{cccc}
0 & 0 & 0 & 0 \\
0 & 0 & 0 & 0 \\
0 & 0 & 0 & 0 \\
0 & 0 & 0 & 0 \\
-0.125 S_{k_{\|}}^{M g O_{3,4}} & 0 & 0 & 0 \\
-0.25 S_{k_{\|}}^{M g O_{4,4}} & -0.375 S_{k_{\|}}^{M g O_{4,5}} & 0 & 0 \\
-0.375 S_{k_{\|}}^{M} g O_{5,4} & -0.5 S_{k_{\|}}^{M g O_{5,5}} & -0.5 S_{k_{\|}}^{M g O 5 F e R i g h t} & 0 \\
0 & -0.5 S_{k_{\|}}^{F e R i g h t M g O 5} & -0.5 S_{k_{\|}}^{F e R i g h t_{1,1}} & -0.5 S_{k_{\|}}^{F e R i g h t_{1,2}} \\
0 & 0 & -0.5 S_{k_{\|}}^{F e R i g h t_{2,1}} & -0.5 S_{k_{\|}}^{F e R i g h t_{2,2}}
\end{array}\right]
\end{aligned}
$$

Green's function:

$$
G_{k_{\|}}=\left(E S_{k_{\|}}-H_{k_{\|}}-U_{k_{\|}}-\Sigma_{k_{\|}}^{\text {left }}-\Sigma_{k_{\|}}^{r i g h t}\right)^{-1}
$$

Broadening matrices:

$$
\begin{gathered}
\Gamma_{k_{\|}}^{l e f t}=i\left(\Sigma_{k_{\|}}^{l e f t}-\Sigma_{k_{\|}}^{l e f t}\right) \\
\Gamma_{k_{\|}}^{r i g h t}=i\left(\Sigma_{k_{\|}}^{r i g h t}-\Sigma_{k_{\|}}^{r i g h t}\right)
\end{gathered}
$$

Current: Current through the entire device is calculated by[101]:

$$
I=\frac{q}{\hbar} \int_{B Z} d k_{\|} \int_{-\infty}^{\infty} d E \operatorname{Trace}\left[\Gamma_{k_{\|}}^{l e f t} G_{k_{\|}} \Gamma_{k_{\|}}^{r i g h t} G_{k_{\|}}^{\dagger}\right]\left(f^{\text {left }}-f^{\text {right }}\right)
$$


where $f^{l e f t}$ and $f^{r i g h t}$ are 1-D Fermi function.

$\begin{array}{lll}1.4884 & 1.4884 & 0.0000 \mathrm{Fe} \\ 0.0000 & 0.0000 & 1.3994 \mathrm{Fe} \\ 1.4884 & 1.4884 & 2.7989 \mathrm{Fe} \\ 0.0000 & 0.0000 & 4.1983 \mathrm{Fe} \\ 1.4884 & 1.4884 & 5.5978 \mathrm{Fe} \\ 0.0000 & 0.0000 & 6.8348 \mathrm{Fe} \\ 0.0000 & 0.0000 & 8.9494 \mathrm{Mg} \\ 1.4884 & 1.4884 & 8.8816 \mathrm{O} \\ 0.0000 & 0.0000 & 11.0636 \mathrm{O} \\ 1.4884 & 1.4884 & 11.0550 \mathrm{Mg} \\ 0.0000 & 0.0000 & 13.1921 \mathrm{Mg} \\ 1.4884 & 1.4884 & 13.1921 \mathrm{O} \\ 0.0000 & 0.0000 & 15.3206 \mathrm{O} \\ 1.4884 & 1.4884 & 15.3291 \mathrm{Mg} \\ 0.0000 & 0.0000 & 17.4348 \mathrm{Mg} \\ 1.4884 & 1.4884 & 17.5025 \mathrm{O} \\ 0.0000 & 0.0000 & 19.6171 \mathrm{Fe} \\ 1.4884 & 1.4884 & 20.8541 \mathrm{Fe} \\ 0.0000 & 0.0000 & 22.2536 \mathrm{Fe} \\ 1.4884 & 1.4884 & 23.6530 \mathrm{Fe} \\ 0.0000 & 0.0000 & 25.0525 \mathrm{Fe} \\ 1.4884 & 1.4884 & 26.4519 \mathrm{Fe}\end{array}$

Figure B.11: On-site atomic coordinates for strained Fe/5 [100] layers of $\mathrm{MgO} / \mathrm{Fe}$ magnetic tunneling junction. 


\section{Appendix C}

\section{Publications}

1. A. Nigam, K. Munira, A. W. Ghosh, S. Wolf, E. Chen, M. R. Stan, 'Self-consistent parameterized physical MTJ compact model for STT-RAM', International Semiconductor Conference 2010.

2. A. Nigam, K. Munira, A.W. Ghosh, S. Wolf, M. R. Stan, 'Model Based Study on Performance and Energy Optimization for STT-RAM', Non-Volatile Memory Workshop 2011.

3. K. Munira, W.A. Soffa, A.W. Ghosh, 'Comparative material issues for fast reliable switching in STT-RAMs', 11th IEEE Conference on Nanotechnology (IEEE-NANO), pp.1403-1408, Aug. 2011.

4. K. Munira, W.H. Butler, A.W. Ghosh, 'A compact model for energy-delay-reliability trade-off studies in single barrier, perpendicular STT-RAM cell', IEEE Transactions on Electron Devices, Vol. 59, Issue. 8. pp.2221- 2226, Aug. 2012.

5. K. Roy, S. Bandyopadhyay, J. Atulasimha, K. Munira, A. W. Ghosh, 'Energy dissipation and switching delay in spin-transfer torque switching of nanomagnets with low-saturation magnetization in the presence of thermal fluctuation', Submitted to Journal of Applied Physics.

6. K. Munira, A.W. Ghosh, 'Deconstruction of the voltage asymmetry between parallel to anti-parallel and anti-parallel to parallel spin-transfer torque switching in in-plane and perpendicular magnetic tunnel junctions', In preparation.

7. K. Munira, S. Nadri, M. Forgues, M. Fashami, K. Roy, J. Atulasimha, S. Bandyopadhyay, A.W. Ghosh, 'Reducing error rates in straintronic multiferroic nanomagnetic logic by pulse shaping', submitted to IEEE Transactions on Electron Devices. 
8. K. Munira, W.H. Butler, A.W. Ghosh, 'Effect of manufacturing variations on energy-delay-reliability trade-off studies in STT-RAM arrays', In preparation.

Book chapters:

1. K. Munira, W.A. Soffa, A.W. Ghosh, 'Material issues for efficient Spin-Transfer Torque RAMs', Nanoelectronic Device Applications, Publisher- CRC Press. 


\section{Bibliography}

[1] G. Atwood. Future directions and challenges for ETox flash memory scaling. IEEE Transactions on Device and Materials Reliability, 4(3):301 - 305, Sept. 2004.

[2] R. Baumann. Soft errors in advanced computer systems. IEEE Design Test of Computers, 22(3):258 - 266, May 2005.

[3] D.J. Frank, R.H. Dennard, E. Nowak, P.M. Solomon, Y. Taur, and Philip Wong Hon-Sum. Device scaling limits of Si MOSFETs and their application dependencies. Proceedings of the IEEE, 89(3):259 -288 , Mar. 2001.

[4] V.V. Zhirnov, J.A. Hutchby, G.I. Bourianoff, and J.E. Brewer. Emerging research memory and logic technologies. IEEE Circuits and Devices Magazine, 21(3):47-51, 2005.

[5] S. A. Wolf, D. D. Awschalom, R. A. Buhrman, J. M. Daughton, S. von Molnar, M. L. Roukes, A. Y. Chtchelkanova, and D. M. Treger. Spintronics: A spin-based electronics vision for the future. Science, 294(5546):1488-1495, 2001.

[6] V.V. Zhirnov, R.K. Cavin III, J.A. Hutchby, and G.I. Bourianoff. Limits to binary logic switch scaling - a gedanken model. Proceedings of the IEEE, 91(11):1934 - 1939, nov 2003.

[7] Sayeef Salahuddin and Supriyo Datta. Interacting systems for self-correcting low power switching. Applied Physics Letters, 90(9):093503, 2007.

[8] E. Hirota, H. Sakakima, and K. Inomata. Giant Magneto-Resistance Devices. Springer, 2002.

[9] M. Julliere. Tunneling between ferromagnetic films. Physics Letters A, 54(3):225226, 1975.

[10] J. M. Slaughter et al. Fundamentals of MRAM technology. Journal of Superconductivity, 15:1, 2002.

[11] J. C. Slonczewski. Current-driven excitation of magnetic multilayers. Journal of Magnetism and Magnetic Materials, 159(1-2):L1 - L7, 1996.

[12] L. Berger. Emission of spin waves by a magnetic multilayer traversed by a current. Phys. Rev. B, 54(13):9353-9358, Oct 1996.

[13] Kuntal Roy, Supriyo Bandyopadhyay, and Jayasimha Atulasimha. Hybrid spintronics and straintronics: A magnetic technology for ultra low energy computing and signal processing. Applied Physics Letters, 99(6):063108, 2011.

[14] G. H. Bernstein, A. Imre, V. Metlushko, A. Orlov, L. Zhou, L. Ji, G. Csaba, and W. Porod. Magnetic QCA systems. Microelectronics Journal, 36:619-624, 2005.

[15] J. Atulasimha and S. Bandyopadhyay. Bennett clocking of nanomagnetic logic using multiferroic single-domain nanomagnets. Applied Physics Letters, 97(17):173105, 2010.

[16] Mohammad Salehi Fashami, Jayasimha Atulasimha, and Supriyo Bandyopadhyay. Magnetization dynamics, throughput and energy dissipation in a universal multiferroic nanomagnetic logic gate with fan-in and fan-out. Nanotechnology, 23(10):105201, 2012. 
[17] C. H. Bennett. The thermodynamics of computation - a review. International Journal of Theoretical Physics, 21:905-940, 1982.

[18] M.T. Niemier, X.S. Hu, M. Alam, G. Bernstein, W. Porod, M. Putney, and J. DeAngelis. Clocking structures and power analysis for nanomagnet-based logic devices. In 2007 ACM/IEEE International Symposium on Low Power Electronics and Design (ISLPED), pages 26 -31, aug. 2007.

[19] J. Das, S.M. Alam, and S. Bhanja. Low power magnetic quantum cellular automata realization using magnetic multi-layer structures. IEEE Journal on Emerging and Selected Topics in Circuits and Systems, 1(3):267 -276, sept. 2011.

[20] Behtash B. Behin-Aein, D. Datta, S. Salahuddin, and S. Datta. Proposal for an all-spin logic device with built-in memory. Nature Nanotechnology, 5(4):266-270, 2010.

[21] Mohammad Salehi Fashami, Kuntal Roy, Jayasimha Atulasimha, and Supriyo Bandyopadhyay. Magnetization dynamics, bennett clocking and associated energy dissipation in multiferroic logic. Nanotechnology, 22(15):155201, 2011.

[22] K. Roy, S. Bandyopadhyay, and J. Atulasimha. A single element universal gate - NAND/NOR implemented with MTJs. In preparation.

[23] Kuntal Roy, Supriyo Bandyopadhyay, and Jayasimha Atulasimha. Switching dynamics of a magnetostrictive single-domain nanomagnet subjected to stress. Phys. Rev. B, 83:224412, Jun 2011.

[24] International technology roadmap for semiconductors, 2010.

[25] K. Munira, W. H. Butler, and A.W. Ghosh. A quasi-analytical model for energy-delay-reliability tradeoff studies during write operations in perpendicular stt-ram cell. IEEE Transactions on Electron Devices, 59:1-6, 2012.

[26] Alan Kalitsov, Mairbek Chshiev, Ioannis Theodonis, Nicholas Kioussis, and W. H. Butler. Spintransfer torque in magnetic tunnel junctions. Phys. Rev. B, 79:174416, May 2009.

[27] A. Kalitsov, I. Theodonis, N. Kioussis, M. Chshiev, W. H. Butler, and A. Vedyayev. Spin-polarized current-induced torque in magnetic tunnel junctions. Journal of Applied Physics, 99(8):08G501, 2006.

[28] J. C. Slonczewski. Conductance and exchange coupling of two ferromagnets separated by a tunneling barrier. Phys. Rev. B, 39(10):6995-7002, Apr 1989.

[29] M. D. Stiles and A. Zangwill. Anatomy of spin-transfer torque. Phys. Rev. B, 66(1):014407, Jun 2002.

[30] G. Finocchio, M. Carpentieri, B. Azzerboni, L. Torres, E. Martinez, and L. Lopez-Diaz. Micromagnetic simulations of nanosecond magnetization reversal processes in magnetic nanopillar. Journal of Applied Physics, 99(8):08G522, 2006.

[31] Dmytro Apalkov, Mahendra Pakala, and Yiming Huai. Micromagnetic simulation of spin transfer torque switching by nanosecond current pulses. Journal of Applied Physics, 99(8):08B907, 2006.

[32] R. G. Parr and W. Yang. Density-functional theory of atoms and molecules. Oxford University Press, 1989.

[33] D. Kienle, J. I. Cerda, and A. W. Ghosh. Extended h[u-umlaut]ckel theory for band structure, chemistry, and transport. i. carbon nanotubes. Journal of Applied Physics, 100(4):043714, 2006.

[34] D. Kienle, K. H. Bevan, G.-C. Liang, L. Siddiqui, J. I. Cerda, and A. W. Ghosh. Extended h[uumlaut]ckel theory for band structure, chemistry, and transport. ii. silicon. Journal of Applied Physics, 100(4):043715, 2006.

[35] J. Cerda and F. Soria. Accurate and transferable extended hückel-type tight-binding parameters. Phys. Rev. B, 61:7965-7971, Mar 2000. 
[36]

[37] J. M Soler, Emilio Artacho, Julian D Gale, Alberto Garcia, Javier Junquera, Pablo Ordejan, and Daniel Sanchez-Portal. The SIESTA method for ab initio order- N materials simulation. Journal of Physics: Condensed Matter, 14(11):2745, 2002.

[38] Reily Rocha. Theoretical and Computational Aspects of electronic transport at the Nanoscale. PhD thesis, University of Dublin, Trinity College, 2007.

[39] Alexandre R. Rocha, Vctor M. Garcasurez, Steve W. Bailey, Colin J. Lambert, Jaime Ferrer, and Stefano Sanvito. Towards molecular spintronics. Nature Materials, 4:335 - 339, 2005.

[40] W. H. Butler. Tunneling magnetoresistance from a symmetry filtering effect. Science and Technology of Advanced Materials, 9(1):014106, 2008.

[41] Ivan Rungger, Oleg Mryasov, and Stefano Sanvito. Resonant electronic states and i-v curves of Fe/MgO/Fe(100) tunnel junctions. Phys. Rev. B, 79:094414, Mar 2009.

[42] Ivan Rungger, Alexandre Reily Rocha, Oleg Mryasov, Olle Heinonen, and Stefano Sanvito. Bias dependent TMR in $\mathrm{Fe} / \mathrm{MgO} / \mathrm{Fe}(100)$ tunnel junctions. MRS Proceedings, 941:0941-Q01-03, 2006.

[43] Ivan Rungger, Alexandre Reily Rocha, Oleg Mryasov, Olle Heinonen, and Stefano Sanvito. Electronic transport through $\mathrm{Fe} / \mathrm{MgO} / \mathrm{Fe}$ tunnel junctions. Journal of Magnetism and Magnetic Materials, 316:481-483, 2007.

[44] Zhitao Diao, Dmytro Apalkov, Mahendra Pakala, Yunfei Ding, Alex Panchula, and Yiming Huai. Spin transfer switching and spin polarization in magnetic tunnel junctions with $\mathrm{MgO}$ and Alo[sub x] barriers. Applied Physics Letters, 87(23):232502, 2005.

[45] Shinji Yuasa, Taro Nagahama, Akio Fukushima, Yoshishige Suzuki, and Koji Ando. Giant roomtemperature magnetoresistance in single-crystal $\mathrm{Fe} / \mathrm{MgO} / \mathrm{Fe}$ magnetic tunnel junctions. Natute Materials, 4:868 - 871, 2004.

[46] Stuart S. P. Parkin, Christian Kaiser, Alex Panchula, Philip M. Rice, Brian Hughes, Mahesh Samant, and See-Hun Yang. Giant tunnelling magnetoresistance at room temperature with MgO (100) tunnel barriers. Nature Materials, 12:862 - 867, 2004.

[47] Ahmet Ali Yanik, Gerhard Klimeck, and Supriyo Datta. Quantum transport with spin dephasing: A nonequlibrium Green's function approach. Phys. Rev. B, 76:045213, Jul 2007.

[48] Supriyo Datta. Electronic transport in memoscopic systems. Cambride University Press, 1995.

[49] John G. Simmons. Low voltage current voltage relationship of tunnel junctions. 34(1):238-239, 1963.

[50] J. C. Slonczewski. Currents, torques, and polarization factors in magnetic tunnel junctions. Phys. Rev. $B, 71: 024411$, January 2005 .

[51] D. Datta, B. Behin-Aein, S. Salahuddin, and S. Datta. Voltage asymmetry of spin-transfer torque. arXiv:0910.2489, 2009.

[52] J. Z. Sun. Spin-current interaction with a monodomain magnetic body: A model study. Phys. Rev. B, 62(1):570-578, Jul 2000.

[53] W. H. Butler, T. Mewes, C. K. A. Mewes, P. B. Visscher, W. H. Rippard, S. E. Russek, and Ranko Heindl. Switching distributions for perpendicular spin-torque devices within the macrospin approximation. arXiv:1202.2621v1.

[54] Hitoshi Kubota, Akio Fukushima, Kay Yakushiji, Taro Nagahama, Shinji Yuasa, Koji Ando, Hiroki Maehara, Yoshinori Nagamine, Koji Tsunekawa, David D. Djayaprawira, Naoki Watanabe, and Yoshishige Suzuki. Quantitative measurement of voltage dependence of spin-transfer torque in MgObased magnetic tunnel junctions. Nat Phys, 4(1):37-41, January 2001. 
[55] Zhitao Diao, Alex Panchula, Yunfei Ding, Mahendra Pakala, Shengyuan Wang, Zhanjie Li, Dmytro Apalkov, Hideyasu Nagai, Alexander Driskill-Smith, Lien-Chang Wang, Eugene Chen, and Yiming Huai. Spin transfer switching in dual mgo magnetic tunnel junctions. Applied Physics Letters, 90(13):132508, 2007.

[56] Yiming Huai, Frank Albert, Paul Nguyen, Mahendra Pakala, and Thierry Valet. Observation of spin-transfer switching in deep submicron-sized and low-resistance magnetic tunnel junctions. Applied Physics Letters, 84(16):3118-3120, 2004.

[57] Masahiko Nakayama, Tadashi Kai, Naoharu Shimomura, Minoru Amano, Eiji Kitagawa, Toshihiko Nagase, Masatoshi Yoshikawa, Tatsuya Kishi, Sumio Ikegawa, and Hiroaki Yoda. Spin transfer switching in $\mathrm{TbCoFe} / \mathrm{CoFeB} / \mathrm{MgO} / \mathrm{CoFeB} / \mathrm{TbCoFe}$ magnetic tunnel junctions with perpendicular magnetic anisotropy. Journal of Applied Physics, 103(7):07A710 -07A713, Apr 2008.

[58] S. Ikeda, K. Miura, H. Yamamoto, K. Mizunuma, H.D. Gan, M. Endo, S. Kanai, J. Hayakawa, F. Matsukura, and $\mathrm{H}$. Ohno. A perpendicular-anisotropy $\mathrm{CoFeB}-\mathrm{MgO}$ magnetic tunnel junction. Nature Materials, 9(1476-1122):721-724, September 2010.

[59] M.Chshiev, I. Theodonis, A. Kalitsov, N. Kioussis, and W.H. Butler. Voltage dependence of spin transfer torque in magnetic tunnel junctions. IEEE Transactions on Magnetics, 44(11):25432546, November 2008.

[60] Z. Li, S. Zhang, Z. Diao, Y. Ding, X. Tang, D. M. Apalkov, Z. Yang, K. Kawabata, and Y. Huai. Perpendicular spin torques in magnetic tunnel junctions. Phys. Rev. Lett., 100:246602, Jun 2008.

[61] Shunichi Hashimoto, Yoshitaka Ochiai, and Koichi Aso. Perpendicular magnetic anisotropy in sputtered CoPd alloy films. Japanese Journal of Applied Physics, 28(Part 1, No. 9):1596-1599, 1989.

[62] Darren Smith, Vishal Parekh, Chunsheng E, Shishan Zhang, Wolfgang Donner, T. Randall Lee, Sakhrat Khizroev, and Dmitri Litvinov. Magnetization reversal and magnetic anisotropy in patterned Co/Pd multilayer thin films. Journal of Applied Physics, 103(2):023920, 2008.

[63] Yiming Huai, Dmytro Apalkov, Zhitao Diao, Yunfei Ding, Alex Panchula, Mahendra Pakala, LienChang Wang, and Eugene Chen. Structure, materials and shape optimization of magnetic tunnel junction devices: Spin-transfer switching current reduction for future magnetoresistive random access memory application. Japanese Journal of Applied Physics, 45(5A):3835-3841, 2006.

[64] E. Chen, D. Apalkov, Z. Diao, A. Driskill-Smith, D. Druist, D. Lottis, V. Nikitin, X. Tang, S. Watts, S. Wang, S.A. Wolf, A.W. Ghosh, J.W. Lu, S.J. Poon, M. Stan, W.H. Butler, S. Gupta, C. Mewes, T. Mewes, and P.B. Visscher. Advances and future prospects of spin-transfer torque random access memory. IEEE Transactions on Magnetics, 46(6):1873 -1878, june 2010.

[65] K. Munira and A.W. Ghosh. Energy-efficient switching in stt-ram using higher polarized ferromagnetic layers. To be published.

[66] J.G. Simmons. Generalized formula for the electric tunnel effect between similar electrodes separated by a thin insulating film. J Appl Phys, 34:1793-1803, 1963.

[67] J.Z. Sun. Spin angular momentum transfer in current-perpendicular nanomagnetic junctions. IBM $J$. RES. \& DEV., 50(1):81-100, 2006.

[68] S. Mangin, D. Ravelosona, J.A. Katine, M.J. Carey, B.D. Terris, and E.E. Fullerton. Current-induced magnetization reversal in nanopillars with perpendicular anisotropy. Nat Mater, 5:210-215, 2006.

[69] T. Klemmer, D. Hoydick, H. Okumura, B. Zhang, and W. A. Soffa. Magnetic hardening and coercivity mechanisms in L10 ordered FePd ferromagnets. Scripta Metallurgica et Materialia, 33(10-11):1793 1805, 1995. Proceedings of an Acta Metallurgica Meeting on Novel Magnetic Structures and Properties. 
[70] A. Natarajarathinam, Z.R. Tadisina, and S. Gupta. Partial perpendicular anisotropy of CoFeB with vanadium capping. In AVS 57th International Symposium $\mathcal{E}$ Exhibition, Magnetic Interfaces and Nanostructures, 2010.

[71] Hiroaki Sukegawa, Shinya Kasai, Takao Furubayashi, Seiji Mitani, and Koichiro Inomata. Spin-transfer switching in an epitaxial spin-valve nanopillar with a full-heusler $\mathrm{Co}[\mathrm{sub} 2] \mathrm{FeAl}[\mathrm{sub}$ 0.5] $\mathrm{Si}[\operatorname{sub} 0.5]$ alloy. Applied Physics Letters, 96(4):042508, 2010.

[72] R. C. Hall. Single crystal anisotropy and magnetostriction constants of several ferromagnetic materials including alloys of NiFe, SiFe, AlFe, CoNi, and CoFe. Journal of Applied Physics, 30(6):816-819, 1959.

[73] Naoki Nishimura, Tadahiko Hirai, Akio Koganei, Takashi Ikeda, Kazuhisa Okano, Yoshinobu Sekiguchi, and Yoshiyuki Osada. Magnetic tunnel junction device with perpendicular magnetization films for high-density magnetic random access memory. Journal of Applied Physics, 91(8):5246-5249, 2002 .

[74] H. Shima, K. Oikawa, A. Fujita, K. Fukamichi, K. Ishida, S. Nakamura, and T. Nojima. Magnetocrystalline anisotropy energy in l10-type copt single crystals. Journal of Magnetism and Magnetic Materials, 290-291(Part 1):566 - 569, 2005. Proceedings of the Joint European Magnetic Symposia (JEMS' 04).

[75] Manli Ding, Yishen Cui, Jiwei Lu, Tim Mewes, and Joseph Poon. Amorphous Gd-Fe-Co as prospective material for perpendicular stt-mram. In APS March Meeting, 2011.

[76] Brij Bihari Lal, Sudhir S. Malhotra, and Michael A. Russak. Magnetic recording medium having a cocr alloy interlayer of a low saturation, Jul 13, 1999.

[77] A. Raychowdhury, D. Somasekhar, T. Karnik, and V. De. Design space and scalability exploration of 1T-1STT MTJ memory arrays in the presence of variability and disturbances. In 2009 IEEE International Electron Devices Meeting (IEDM), pages 1-4, December 2009.

[78] C. Augustine, A. Raychowdhury, D. Somasekhar, J. Tschanz, V. De, and K. Roy. Design space exploration of typical STT MTJ stacks in memory arrays in the presence of variability and disturbances. IEEE Transactions on Electron Devices, 58(12):4333 -4343, December 2011.

[79] Xiaobin Wang, Yuankai Zheng, Haiwen Xi, and Dimitar Dimitrov. Thermal fluctuation effects on spin torque induced switching: Mean and variations. Journal of Applied Physics, 103(3):034507, 2008.

[80] Y. Huai. Spin-transfer torque MRAM (STT-MRAM): Challenges and prospects. AAPPS Bulletin,, 18(6):36, December 2008.

[81] William Fuller Brown. Thermal fluctuations of a single-domain particle. Phys. Rev., 130:1677-1686, Jun 1963.

[82] R. P. Cowburn, D. K. Koltsov, A. O. Adeyeye, M. E. Welland, and D. M. Tricker. Single-domain circular nanomagnets. Phys. Rev. Lett., 83:1042-1045, Aug 1999.

[83] G Bertotti, C Serpico, and ID Mayergoyz. Nonlinear Magnetization Dynamics in Nanosystems. Elsevier Science, 2008.

[84] S Chikazumi. Physics of Magnetism. Wiley, 1694.

[85] K. Ried, M. Schnell, F. Schatz, M. Hirscher, B. Ludescher, W. Sigle, and H. Kronmller. Crystallization behaviour and magnetic properties of magnetostrictive TbDyFe films. physica status solidi (a), 167(1):195-208, 1998.

[86] J Walowski, M Djordjevic Kaufmann, B Lenk, C Hamann, J McCord, and M Manzenberg. Intrinsic and non-local gilbert damping in polycrystalline nickel studied by $\mathrm{Ti}$ : sapphire laser fs spectroscopy. Journal of Physics D: Applied Physics, 41(16):164016, 2008. 
[87] Rick Kellogg and Alison Flatau. Experimental investigation of Terfenol-D's elastic modulus. Journal of Intelligent Material Systems and Structures, 19(5):583-595, 2008.

[88] H. Zheng, J. Wang, S. E. Lofland, Z. Ma, L. Mohaddes-Ardabili, T. Zhao, L. Salamanca-Riba, S. R. Shinde, S. B. Ogale, F. Bai, D. Viehland, Y. Jia, D. G. Schlom, M. Wuttig, A. Roytburd, and R. Ramesh. Multiferroic batio3-cofe2o4 nanostructures. Science, 303(5658):661-663, 2004.

[89] G. D. Fuchs, I. N. Krivorotov, P. M. Braganca, N. C. Emley, A. G. F. Garcia, D. C. Ralph, and R. A. Buhrman. Adjustable spin torque in magnetic tunnel junctions with two fixed layers. Applied Physics Letters, 86(15):152509, 2005.

[90] G. Feng, Sebastiaan van Dijken, and J. M. D. Coey. Mgo-based double barrier magnetic tunnel junctions with thin free layers. Journal of Applied Physics, 105(7):07C926, 2009.

[91] S. Colis, G. Gieres, L. Bar, and J. Wecker. Low tunnel magnetoresistance dependence versus bias voltage in double barrier magnetic tunnel junction. Applied Physics Letters, 83(5):948-950, 2003.

[92] Yan Wang, Zhong-Yi Lu, X.-G. Zhang, and X. F. Han. First-principles theory of quantum well resonance in double barrier magnetic tunnel junctions. Phys. Rev. Lett., 97(8):087210, Aug 2006.

[93] Jun Yang, Jun Wang, Z. M. Zheng, D. Y. Xing, and C. R. Chang. Quantum oscillations of tunneling magnetoresistance in magnetic tunnel junctions. Phys. Rev. B, 71(21):214434, Jun 2005.

[94] Z.M. Zeng, H.X. Wei, L.X. Jiang, G.X. Du, W.S. Zhan, and X.F. Han. High magnetoresistance in CoFe-B-based double barrier magnetic tunnel junction. Journal of Magnetism and Magnetic Materials, 303(2):e219 - e222, 2006. The 6th International Symposium on Physics of Magnetic Materials.

[95] A. Vedyayev, N. Ryzhanova, B. Dieny, and N. Strelkov. Resonant spin-torque in double barrier magnetic tunnel junctions. Physics Letters A, 355(3):243 - 246, 2006.

[96] Niladri Narayan Mojumder, Charles Augustine, Dmitri E. Nikonov, and Kaushik Roy. Effect of quantum confinement on spin transport and magnetization dynamics in dual barrier spin transfer torque magnetic tunnel junctions. Journal of Applied Physics, 108(10):104306, 2010.

[97] Lixian Jiang, Hiroshi Naganuma, Mikihiko Oogane, and Yasuo Ando. Large tunnel magnetoresistance of $1056 \%$ at room temperature in mgo based double barrier magnetic tunnel junction. Applied Physics Express, 2(8):083002, 2009.

[98] S. Datta. Enrico fermi. In Proc. Of Inter. School of Phys, Italy, 2004.

[99] Chungsheng Liu. Theoretical investigation of the intrinsic Gilbert damping parameter using firstprinciples and tight-binding models. PhD thesis, University of Alabama, 2009.

[100] M P Lopez Sancho, J M Lopez Sancho, and J Rubio. Quick iterative scheme for the calculation of transfer matrices: application to mo (100). Journal of Physics F: Metal Physics, 14(5):1205, 1984.

[101] Derek Waldron, Lei Liu, and Hong Guo. Ab initio simulation of magnetic tunnel junctions. Nanotechnology, 18(42):424026, 2007. 